\title{
Catalytic Asymmetric Synthesis of Atropisomeric Nitrones: Versatile Intermediate for Axially Chiral Biaryls
}

Wenjing Guo, Jian Gu and Zhenhua Gu*

Department of Chemistry, Center for Excellence in Molecular Synthesis, and Hefei National Laboratory for Physical Sciences at the Microscale, University of Science and Technology of China, 96 Jinzhai Road, Hefei, Anhui 230026, P.R. China

Ocean College, Minjiang University, Fuzhou, Fujian, 350108, P. R. China zhgu@ustc.edu.cn 


\section{Table of Contents}

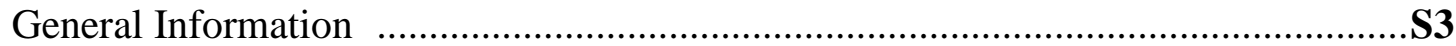

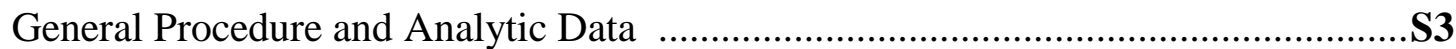

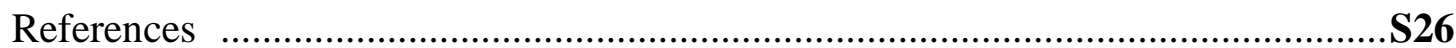

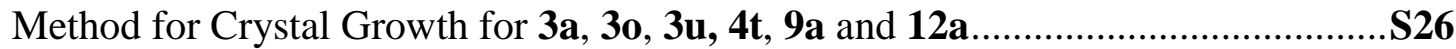

Crystal Data and Structure Refinement ….......................................................... S27

Thermal Ellipsoid Plot for the Crystal Structure of 3a n..........................................S33

Thermal Ellipsoid Plot for the Crystal Structure of $\mathbf{3 0}$............................................S34

Thermal Ellipsoid Plot for the Crystal Structure of 3u' .......................................S35

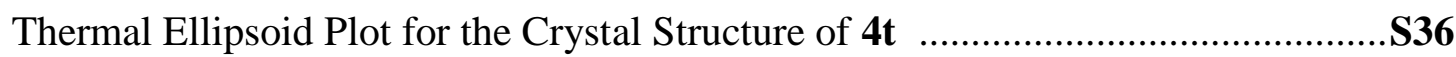

Thermal Ellipsoid Plot for the Crystal Structure of 9a …..........................................337

Thermal Ellipsoid Plot for the Crystal Structure of 12a .........................................S38

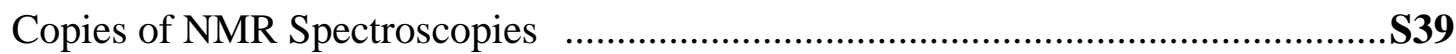

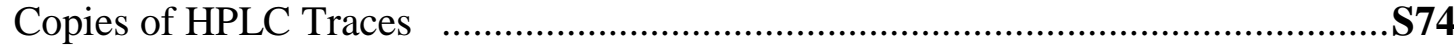




\section{General Information}

Nuclear magnetic resonances were recorded on Bruker-400 MHz or Bruker-500 MHz instruments. Reference values for residual solvents were taken as $\delta=7.26 \mathrm{ppm}\left(\mathrm{CDCl}_{3}\right), 2.50$ ppm (DMSO-d $\left.\mathrm{d}_{6}\right), 7.16 \mathrm{ppm}\left(\mathrm{C}_{6} \mathrm{D}_{6}\right)$ for ${ }^{1} \mathrm{H}$ NMR; $\delta=77.00$ ppm $\left(\mathrm{CDCl}_{3}\right), 39.52 \mathrm{ppm}$ (DMSO$\left.d_{6}\right), 128.06 \mathrm{ppm}\left(\mathrm{C}_{6} \mathrm{D}_{6}\right)$ for ${ }^{13} \mathrm{C}$ NMR. High resolution mass spectral analysis (HRMS) was performed on Waters XEVO G2 Q-TOF (Waters Corporation). All reactions were performed under an inert atmosphere of dry nitrogen in flame-dried glassware unless otherwise stated. The low temperature reaction bath uses ethanol as the medium, and the temperature also refers to the temperature of ethanol bath. The temperature above room temperature is heated by using oil as the medium, and the temperature also refers to the temperature of oil bath. Dichloromethane were distilled over calcium hydride and stored in a solvent bottle containing dried molecular sieves. Diethyl ether were distilled over sodium in the presence of benzophenone under atmosphere of nitrogen. Toluene and dichloroethane were distilled over calcium hydride under atmosphere of nitrogen. 1,4-Dioxane and acetonitrile were super dry solvents stored in the presence of molecular sieves. The cyclic diaryliodoniums with triflate anion were synthesized according to the reported literature. ${ }^{1}$

\section{General Procedure for the Synthesis of 3 (Procedure A)}

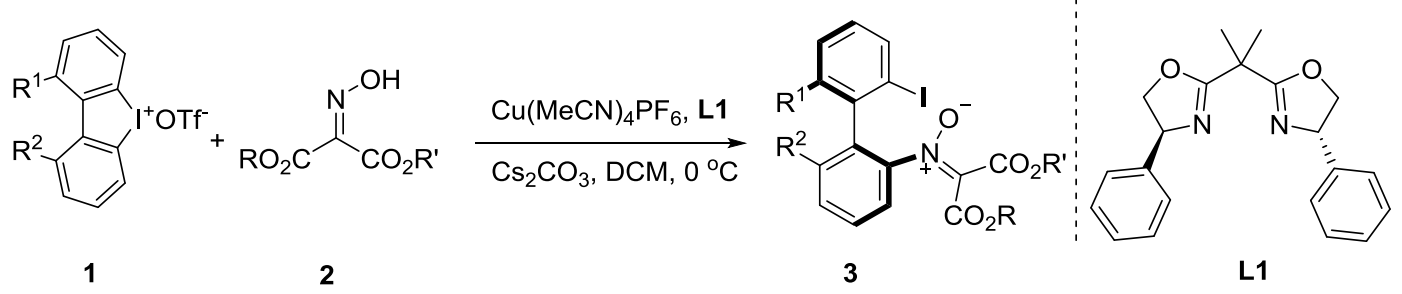

To a Schlenk tube containing $\mathrm{Cu}(\mathrm{MeCN})_{4} \mathrm{PF}_{6}(5.0 \mathrm{~mol} \%),(\mathrm{S}, \mathrm{S})-\mathrm{Ph}-\mathrm{Box}(6.0 \mathrm{~mol} \%)(\mathrm{Cu} / \mathrm{L}$ $=1: 1.2$, unless otherwise stated) was added $\mathrm{CH}_{2} \mathrm{Cl}_{2}(2.0 \mathrm{~mL})$ under nitrogen at room temperature. After being stirred for $1 \mathrm{~h}$, the solvent was removed under vacuum. To above Schlenk tube was added 1 ( $0.100 \mathrm{mmol}, 1.00$ equiv), 2 ( $0.125 \mathrm{mmol}, 1.25$ equiv), $\mathrm{Cs}_{2} \mathrm{CO}_{3}(0.150$ 
mmol, 1.50 equiv) followed by $\mathrm{CH}_{2} \mathrm{Cl}_{2}(0.05 \mathrm{M})$ under nitrogen at $0{ }^{\circ} \mathrm{C}$ in ethanol bath. After being stirred for another $1 \mathrm{~h}$ (monitored by TLC) at $0{ }^{\circ} \mathrm{C}$, the solvent was removed and the residue was purified by flash column chromatography on silica gel to afford the desired product 3.

Compound 3a was prepared following the Procedure A

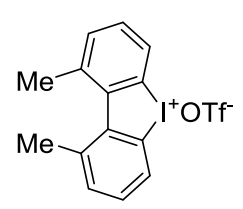

$1 \mathbf{a}$

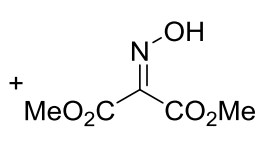

$2 \mathbf{a}$

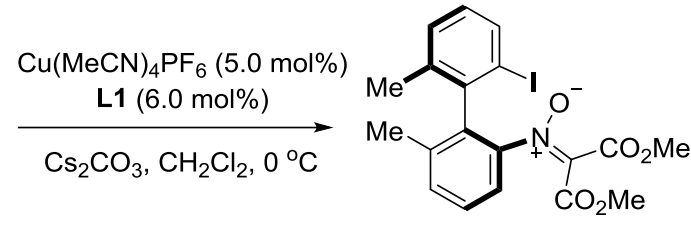

$3 \mathbf{a}$

The reaction of $1 \mathbf{a}(45.6 \mathrm{mg}, 0.100 \mathrm{mmol}, 1.00$ equiv), $\mathbf{2 a}(20.1 \mathrm{mg}, 0.125 \mathrm{mmol}, 1.25$ equiv), $\mathrm{Cs}_{2} \mathrm{CO}_{3}$ (48.9 mg, $0.150 \mathrm{mmol}, 1.50$ equiv), $\mathrm{Cu}(\mathrm{MeCN})_{4} \mathrm{PF}_{6}(1.9 \mathrm{mg}, 5.0 \mathrm{~mol} \%)$ and $\mathbf{~ L 1}(2.0$ $\mathrm{mg}, 6.0 \mathrm{~mol} \%)$ in $\mathrm{CH}_{2} \mathrm{Cl}_{2}(2.0 \mathrm{~mL})$ at $0{ }^{\circ} \mathrm{C}$ in ethanol bath for $1 \mathrm{~h}$ afforded $3 \mathrm{a}(46.1 \mathrm{mg}, 99 \%$, 98\% ee, pale yellow solid). $[\alpha]_{D}^{20}=+46\left(\mathrm{c}=1.00, \mathrm{CHCl}_{3}\right)$. HPLC conditions: Chiralpak AD$\mathrm{H}$, isopropanol/hexane $=10: 90$, flow: $1.0 \mathrm{~mL} / \mathrm{min}, \lambda=254 \mathrm{~nm} .{ }^{1} \mathbf{H} \mathbf{~ N M R}\left(500 \mathrm{MHz}, \mathrm{CDCl}_{3}\right) \delta$ $7.74(\mathrm{~d}, J=8.0 \mathrm{~Hz}, 1 \mathrm{H}), 7.46(\mathrm{~d}, J=7.5 \mathrm{~Hz}, 1 \mathrm{H}), 7.42(\mathrm{t}, J=7.5 \mathrm{~Hz}, 1 \mathrm{H}), 7.32$ (bs, 1H), 7.22 $(\mathrm{d}, J=8.0 \mathrm{~Hz}, 1 \mathrm{H}), 6.97(\mathrm{t}, J=8.0 \mathrm{~Hz}, 1 \mathrm{H}), 3.78(\mathrm{~s}, 3 \mathrm{H}), 3.71(\mathrm{~s}, 3 \mathrm{H}), 2.05(\mathrm{~s}, 6 \mathrm{H}) .{ }^{13} \mathbf{C}$ NMR $\left(101 \mathrm{MHz}, \mathrm{CDCl}_{3}\right) \delta 160.7,157.7,143.4,140.6,139.0,138.2,136.9,136.4,133.6,132.0,129.7$, 127.5, 122.1, 103.0, 97.9, 53.0, 52.8, 21.6, 20.7, 19.8. ${ }^{13} \mathbf{C}$ NMR (126 MHz, DMSO- $\left.d_{6}\right) \delta 160.6$, 157.3, 143.1, 139.8, 139.0, 137.7, 136.2, 132.9, 132.1, 129.8, 129.60, 128.0, 122.4, 103.4, 98.4, 53.1, 53.0, 21.2, 20.3, 19.4. ${ }^{13} \mathbf{C}$ NMR $\left(\mathbf{1 0 0}^{\circ} \mathbf{C}, 151 \mathrm{MHz}, \mathrm{DMSO}-d_{6}\right) \delta 160.0,157.1,143.2$, 138.6, 138.1, 137.4, 135.82, 135.75, 132.7, 131.5, 129.13, 129.11, 127.5, 121.8, 100.1, 52.26, 52.21, 20.2, 18.7. HRMS (ESI) m/z: $[\mathrm{M}+\mathrm{Na}]^{+}$Calcd for $\mathrm{C}_{19} \mathrm{H}_{18} \mathrm{INO}_{5} \mathrm{Na}$ 490.0127; Found 490.0124. It should take 1-4 h to get reasonable ${ }^{13} \mathrm{C}$ NMR spectra at the concentration of $\sim 100$ $\mathrm{mg} / \mathrm{mL}\left(\mathrm{CDCl}_{3}\right)$. This phenomenon might be caused by the rotamers due to the slow rotation of C-N single bond. The broad signals in ${ }^{13} \mathrm{C}$ NMR spectroscopies were further confirmed by HSQC and HMBC analysis (see copies of NMR spectroscopies of 3a). 


\section{0 mmol Scale Reaction}<smiles></smiles>

1a

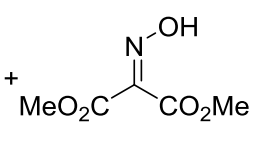

2a

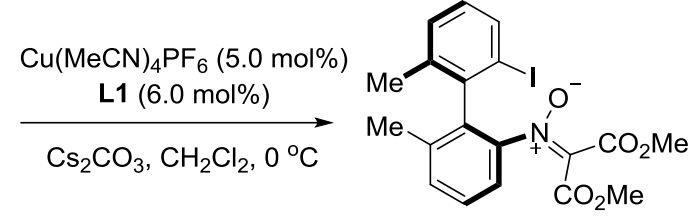

3a

To a $100 \mathrm{~mL}$ Schlenk flask containing $\mathrm{Cu}(\mathrm{MeCN})_{4} \mathrm{PF}_{6}$ (93.2 mg, $\left.5.0 \mathrm{~mol} \%\right),(S, S)-\mathrm{Ph}-\mathrm{Box}$ (100.3 mg, $6.0 \mathrm{~mol} \%$ ) was added $\mathrm{CH}_{2} \mathrm{Cl}_{2}(10.0 \mathrm{~mL})$ under nitrogen at room temperature. After being stirred for $1 \mathrm{~h}$, the solvent was removed under vacuum. To above Schlenk tube was added 1a (2.28 g, 5.00 mmol, 1.00 equiv), $2 \mathrm{a}$ (1.01 g, $6.25 \mathrm{mmol}, 1.25$ equiv), $\mathrm{Cs}_{2} \mathrm{CO}_{3}(2.44 \mathrm{~g}, 7.50$ mmol, 1.50 equiv) followed by $\mathrm{CH}_{2} \mathrm{Cl}_{2}(50.0 \mathrm{~mL})$ under nitrogen at $0{ }^{\circ} \mathrm{C}$ in ethanol bath. After being stirred for another $1 \mathrm{~h}$ (monitored by TLC) at $0{ }^{\circ} \mathrm{C}$, the solvent was removed and the residue was purified by flash column chromatography on silica gel (hexane/ethyl acetate $=5: 1$ ) to afford 3a (2.20 g, 94\%, 97\% ee, pale yellow solid).

Synthesis of Compound $\mathbf{4 a}$

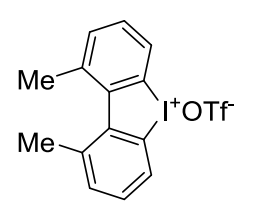

$1 a$

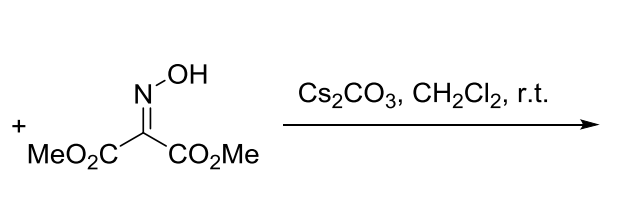

$2 \mathbf{a}$

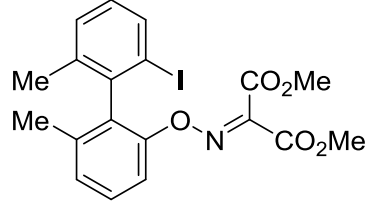

$4 a$

To a Schlenk tube was added 1a (45.6 mg, $0.100 \mathrm{mmol}, 1.00$ equiv), 2a (20.1 mg, 0.125 mmol, 1.25 equiv) and $\mathrm{Cs}_{2} \mathrm{CO}_{3}$ (48.9 mg, $0.150 \mathrm{mmol}, 1.50$ equiv) followed by $\mathrm{CH}_{2} \mathrm{Cl}_{2}$ (2.0 $\mathrm{mL}$ ) under nitrogen at room temperature. After being stirred for $24 \mathrm{~h}$ (monitored by TLC), the solvent was removed and the residue was purified by flash column chromatography on silica gel to afford the desired product $4 \mathbf{4 a}\left(37.8 \mathrm{mg}, 81 \%\right.$, yellow oil). ${ }^{1} \mathbf{H} \mathbf{~ N M R}\left(400 \mathrm{MHz}, \mathrm{CDCl}_{3}\right) \delta$ $7.76(\mathrm{~d}, J=8.0 \mathrm{~Hz}, 1 \mathrm{H}), 7.42(\mathrm{~d}, J=8.0 \mathrm{~Hz}, 1 \mathrm{H}), 7.34(\mathrm{t}, J=8.0 \mathrm{~Hz}, 1 \mathrm{H}), 7.24(\mathrm{~d}, J=7.6 \mathrm{~Hz}$, 
1H), $7.10(\mathrm{~d}, J=7.6 \mathrm{~Hz}, 1 \mathrm{H}), 6.96(\mathrm{t}, J=7.6 \mathrm{~Hz}, 1 \mathrm{H}), 3.89(\mathrm{~s}, 3 \mathrm{H}), 3.47(\mathrm{~s}, 3 \mathrm{H}), 1.98(\mathrm{~s}, 3 \mathrm{H})$, $1.97(\mathrm{~s}, 3 \mathrm{H}) .{ }^{13} \mathrm{C}$ NMR $\left(126 \mathrm{MHz}, \mathrm{CDCl}_{3}\right) \delta 160.0,159.7,154.4,145.6,140.7,138.4,137.3$, 136.3, 131.2, 129.4, 128.98, 128.97, 125.9, 112.3, 101.1, 53.3, 52.6, 21.3, 19.1. HRMS (ESI) $\mathrm{m} / \mathrm{z}:[\mathrm{M}+\mathrm{Na}]^{+}$Calcd for $\mathrm{C}_{19} \mathrm{H}_{18} \mathrm{INO}_{5} \mathrm{Na}$ 490.0127; Found 490.0111 .

Compound $3 f$ was prepared following the Procedure A

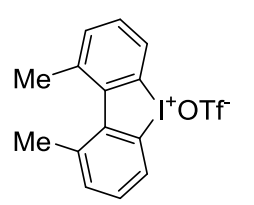

$1 \mathbf{a}$

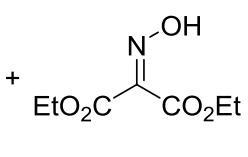

$2 f$

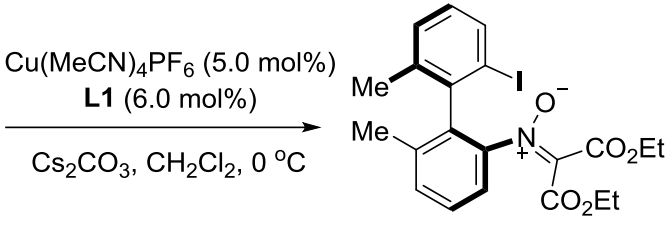

$3 f$

The reaction of $\mathbf{1 a}$ ( $45.6 \mathrm{mg}, 0.100 \mathrm{mmol}, 1.00$ equiv), $\mathbf{2 f}$ ( $23.6 \mathrm{mg}, 0.125 \mathrm{mmol}, 1.25$ equiv), $\mathrm{Cs}_{2} \mathrm{CO}_{3}$ (48.9 mg, $0.150 \mathrm{mmol}, 1.50$ equiv), $\mathrm{Cu}(\mathrm{MeCN})_{4} \mathrm{PF}_{6}(1.9 \mathrm{mg}, 5.0 \mathrm{~mol} \%)$ and $\mathbf{~} \mathbf{1}(2.0$ $\mathrm{mg}, 6.0 \mathrm{~mol} \%)$ in $\mathrm{CH}_{2} \mathrm{Cl}_{2}(2.0 \mathrm{~mL})$ at $0{ }^{\circ} \mathrm{C}$ in ethanol bath for $1 \mathrm{~h}$ afforded $\mathbf{3 f}(47.7 \mathrm{mg}, 96 \%$, 95\% ee, yellow oil). $[\alpha]_{D}^{20}=+35\left(\mathrm{c}=1.04, \mathrm{CHCl}_{3}\right)$. HPLC conditions: Chiralpak AD-H, isopropanol/hexane $=5: 95$, flow: $1.0 \mathrm{~mL} / \mathrm{min}, \lambda=254 \mathrm{~nm} .{ }^{1} \mathbf{H} \mathbf{~ N M R}\left(500 \mathrm{MHz}, \mathrm{CDCl}_{3}\right) \delta 7.73$ $(\mathrm{d}, J=8.0 \mathrm{~Hz}, 1 \mathrm{H}), 7.45(\mathrm{~d}, J=7.5 \mathrm{~Hz}, 1 \mathrm{H}), 7.41(\mathrm{t}, J=7.5 \mathrm{~Hz}, 1 \mathrm{H}), 7.34(\mathrm{bs}, 1 \mathrm{H}), 7.22(\mathrm{~d}, J$ $=8.0 \mathrm{~Hz}, 1 \mathrm{H}), 6.96(\mathrm{t}, J=8.0 \mathrm{~Hz}, 1 \mathrm{H}), 4.24(\mathrm{q}, J=7.0 \mathrm{~Hz}, 2 \mathrm{H}), 4.16(\mathrm{q}, J=7.0 \mathrm{~Hz}, 2 \mathrm{H}), 2.05$ $(\mathrm{s}, 6 \mathrm{H}), 1.22(\mathrm{t}, J=7.0 \mathrm{~Hz}, 3 \mathrm{H}), 1.20(\mathrm{t}, J=7.0 \mathrm{~Hz}, 3 \mathrm{H}) .{ }^{13} \mathbf{C} \mathbf{N M R}\left(126 \mathrm{MHz}, \mathrm{CDCl}_{3}\right) \delta 160.2$, 157.3, 143.4, 140.6, 139.1, 138.1, 137.0, 136.3, 134.1, 131.8, 129.6, 127.4, 122.1, 103.0, 98.0, 62.07, 61.98, 21.6, 20.6, 19.7, 13.7, 13.6. HRMS (ESI) m/z: [M + Na] ${ }^{+}$Calcd for $\mathrm{C}_{21} \mathrm{H}_{22} \mathrm{INO}_{5} \mathrm{Na}$ 518.0440; Found 518.0440.

Compound $\mathbf{3 g}$ was prepared following the Procedure A 
<smiles>Cc1cccc2c1-c1c(C)cccc1[I+]2[O-]</smiles>

1a<smiles>CCOC(=O)C(=O)ON=C(C)OC</smiles>

$2 \mathrm{~g}$

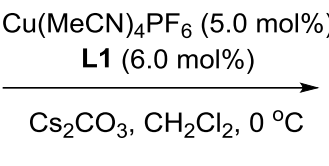

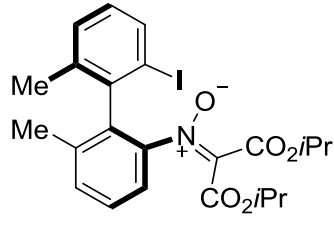

$3 g$

The reaction of $1 \mathbf{a}(45.6 \mathrm{mg}, 0.100 \mathrm{mmol}, 1.00$ equiv), $2 \mathrm{~g}$ ( $27.2 \mathrm{mg}, 0.125 \mathrm{mmol}, 1.25$ equiv), $\mathrm{Cs}_{2} \mathrm{CO}_{3}$ (48.9 mg, $0.150 \mathrm{mmol}, 1.50$ equiv), $\mathrm{Cu}(\mathrm{MeCN})_{4} \mathrm{PF}_{6}(1.9 \mathrm{mg}, 5.0 \mathrm{~mol} \%)$ and $\mathbf{L 1}(2.0$ $\mathrm{mg}, 6.0 \mathrm{~mol} \%)$ in $\mathrm{CH}_{2} \mathrm{Cl}_{2}(2.0 \mathrm{~mL})$ at $0{ }^{\circ} \mathrm{C}$ in ethanol bath for $1 \mathrm{~h}$ afforded $3 \mathrm{~g}(49.9 \mathrm{mg}, 95 \%$, 97\% ee, yellow-brown solid). $[\alpha]_{D}^{20}=+38\left(\mathrm{c}=1.00, \mathrm{CHCl}_{3}\right)$. HPLC conditions: Chiralpak AD-H, isopropanol/hexane $=5: 95$, flow: $1.0 \mathrm{~mL} / \mathrm{min}, \lambda=254 \mathrm{~nm} .{ }^{1} \mathbf{H} \mathbf{~ N M R}\left(500 \mathrm{MHz}, \mathrm{CDCl}_{3}\right)$ $\delta 7.73(\mathrm{~d}, J=8.0 \mathrm{~Hz}, 1 \mathrm{H}), 7.44(\mathrm{~d}, J=7.5 \mathrm{~Hz}, 1 \mathrm{H}), 7.41(\mathrm{t}, J=7.5 \mathrm{~Hz}, 1 \mathrm{H}), 7.34(\mathrm{br}, 1 \mathrm{H}), 7.21$ (d, $J=8.0 \mathrm{~Hz}, 1 \mathrm{H}$ ), 6.95 (t, $J=8.0 \mathrm{~Hz}, 1 \mathrm{H}$ ), 5.10 (hept., $J=6.0 \mathrm{~Hz}, 1 \mathrm{H}$ ), 4.97 (hept., $J=6.0$ $\mathrm{Hz}, 1 \mathrm{H}), 2.05(\mathrm{~s}, 6 \mathrm{H}), 1.25-1.21(\mathrm{~m}, 6 \mathrm{H}), 1.19(\mathrm{~d}, J=6.0 \mathrm{~Hz}, 6 \mathrm{H}) .{ }^{13} \mathbf{C ~ N M R}\left(126 \mathrm{MHz}, \mathrm{CDCl}_{3}\right)$ $\delta 159.6,157.3,156.7,143.3,140.6,139.2,137.9,136.9,136.2,134.5,131.7,129.5,127.3$, 122.1, 103.1, 98.0, 70.0, 69.8, 21.5, 21.3, 21.22, 21.18, 20.6, 19.7. HRMS (ESI) m/z: $[\mathrm{M}+\mathrm{Na}]^{+}$ Calcd for $\mathrm{C}_{23} \mathrm{H}_{26} \mathrm{INO}_{5} \mathrm{Na}$ 546.0753; Found 546.0758.

Compound 3h was prepared following the Procedure A<smiles>Cc1cccc2c1-c1c(C)cccc1[O+]2</smiles>

$1 \mathrm{a}$<smiles>CCCCOC(=O)/C(=N/O)OCC</smiles>

$2 \mathrm{~h}$

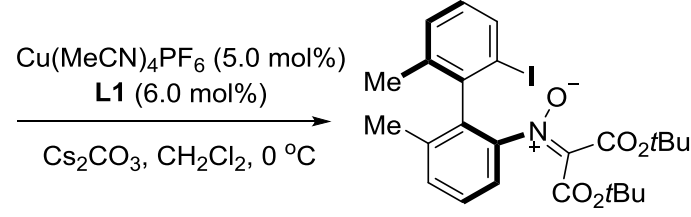

$3 \mathrm{~h}$

The reaction of $1 \mathbf{a}$ ( $45.6 \mathrm{mg}, 0.100 \mathrm{mmol}, 1.00$ equiv), $2 \mathbf{h}$ ( $30.7 \mathrm{mg}, 0.125 \mathrm{mmol}, 1.25$ equiv), $\mathrm{Cs}_{2} \mathrm{CO}_{3}$ (48.9 mg, $0.150 \mathrm{mmol}, 1.50$ equiv), $\mathrm{Cu}(\mathrm{MeCN})_{4} \mathrm{PF}_{6}(1.9 \mathrm{mg}, 5.0 \mathrm{~mol} \%)$ and $\mathbf{L 1}(2.0$ $\mathrm{mg}, 6.0 \mathrm{~mol} \%)$ in $\mathrm{CH}_{2} \mathrm{Cl}_{2}(2.0 \mathrm{~mL})$ at $0{ }^{\circ} \mathrm{C}$ in ethanol bath for $1 \mathrm{~h}$ afforded $\mathbf{3 h}(42.7 \mathrm{mg}, 77 \%$, 95\% ee, yellow solid). $[\alpha]_{D}^{20}=+24\left(\mathrm{c}=1.10, \mathrm{CHCl}_{3}\right)$. Compound $3 \mathrm{~h}$ was metastable upon isolation and characterization. HPLC conditions: Chiralpak AD-H, isopropanol/hexane $=5: 95$, 
flow: $1.0 \mathrm{~mL} / \mathrm{min}, \lambda=254 \mathrm{~nm} .{ }^{1} \mathbf{H}$ NMR $\left(400 \mathrm{MHz}, \mathrm{CDCl}_{3}\right) \delta 7.72(\mathrm{~d}, J=7.6 \mathrm{~Hz}, 1 \mathrm{H}), 7.43-$ $7.37(\mathrm{~m}, 2 \mathrm{H}), 7.31(\mathrm{br}, 1 \mathrm{H}), 7.20(\mathrm{~d}, J=7.6 \mathrm{~Hz}, 1 \mathrm{H}), 6.94(\mathrm{t}, J=7.6 \mathrm{~Hz}, 1 \mathrm{H}), 2.06(\mathrm{~s}, 3 \mathrm{H}), 2.03$ (s, 3H), 1.42 (s, 18H). ${ }^{13} \mathbf{C}$ NMR (126 MHz, $\left.\mathrm{CDCl}_{3}\right) \delta$ 159.3, 156.8, 156.1, 143.2, 140.7, 139.7, 139.0, 138.3, 137.9, 136.9, 136.2, 135.5, 135.1, 131.6, 129.4, 129.3, 127.3, 122.0, 103.3, 97.9, 83.3, 83.1, 27.9, 27.7, 21.7, 20.7, 19.7. HRMS (ESI) m/z: $[\mathrm{M}+\mathrm{Na}]^{+}$Calcd for $\mathrm{C}_{25} \mathrm{H}_{30} \mathrm{INO}_{5} \mathrm{Na}$ 574.1066; Found 574.1074.

Compound 3i was prepared following the Procedure A

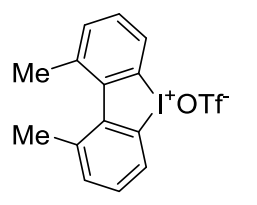

1a

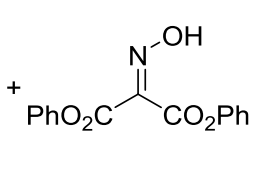

$2 \mathbf{i}$

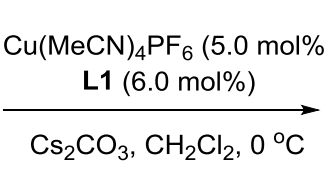

$\mathrm{s}_{2} \mathrm{CO}_{3}, \mathrm{CH}_{2} \mathrm{Cl}_{2}, 0^{\circ} \mathrm{C}$

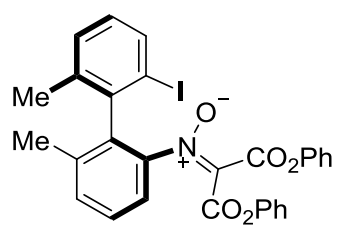

3i

The reaction of $1 \mathbf{a}(45.6 \mathrm{mg}, 0.100 \mathrm{mmol}, 1.00$ equiv), $2 \mathbf{i}$ ( $32.3 \mathrm{mg}, 0.125 \mathrm{mmol}, 1.25$ equiv), $\mathrm{Cs}_{2} \mathrm{CO}_{3}$ (48.9 mg, $0.150 \mathrm{mmol}, 1.50$ equiv), $\mathrm{Cu}(\mathrm{MeCN})_{4} \mathrm{PF}_{6}(1.9 \mathrm{mg}, 5.0 \mathrm{~mol} \%)$ and $\mathbf{L 1}(2.0$ $\mathrm{mg}, 6.0 \mathrm{~mol} \%)$ in $\mathrm{CH}_{2} \mathrm{Cl}_{2}(2.0 \mathrm{~mL})$ at $0{ }^{\circ} \mathrm{C}$ in ethanol bath for $1 \mathrm{~h}$ afforded $3 \mathbf{i}(53.0 \mathrm{mg}, 90 \%$, 97\% ee, brown oil). $[\alpha]_{D}^{20}=+49\left(\mathrm{c}=0.64, \mathrm{CHCl}_{3}\right)$. HPLC conditions: Chiralpak OD-H, isopropanol/hexane $=5: 95$, flow: $1.0 \mathrm{~mL} / \mathrm{min}, \lambda=254 \mathrm{~nm} .{ }^{1} \mathbf{H} \mathbf{N M R}\left(500 \mathrm{MHz}, \mathrm{CDCl}_{3}\right) \delta 7.77$ $(\mathrm{d}, J=7.5 \mathrm{~Hz}, 1 \mathrm{H}), 7.46-7.42(\mathrm{~m}, 3 \mathrm{H}), 7.38-7.35(\mathrm{~m}, 4 \mathrm{H}), 7.25-7.22(\mathrm{~m}, 3 \mathrm{H}), 7.14(\mathrm{br}, 4 \mathrm{H})$, $6.97(\mathrm{t}, J=7.5 \mathrm{~Hz}, 1 \mathrm{H}), 2.11(\mathrm{~s}, 3 \mathrm{H}), 2.07$ (s, 3H). ${ }^{13} \mathbf{C} \mathbf{~ N M R}\left(126 \mathrm{MHz}, \mathrm{CDCl}_{3}\right) \delta 158.7,155.8$, 150.0, 149.6, 143.4, 140.8, 139.1, 138.4, 136.9, 136.4, 133.6, 132.3, 129.8, 129.49, 129.45, 127.6, 126.5, 126.4 122.1, 121.3, 121.2, 98.1, 21.8, 20.9, 19.9. HRMS (ESI) m/z: $[\mathrm{M}+\mathrm{Na}]^{+}$ Calcd for $\mathrm{C}_{29} \mathrm{H}_{22} \mathrm{INO}_{5} \mathrm{Na}$ 614.0440; Found 614.0450.

Compound $\mathbf{3 j}$ was prepared following the Procedure A 


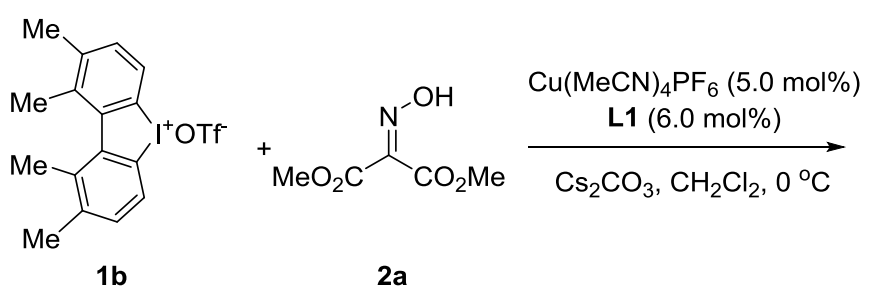

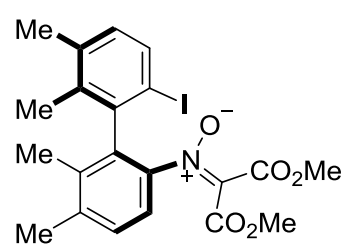

3j

The reaction of $\mathbf{1 b}$ ( $48.4 \mathrm{mg}, 0.100 \mathrm{mmol}, 1.00$ equiv), $\mathbf{2 a}$ (20.1 mg, $0.125 \mathrm{mmol}, 1.25$ equiv), $\mathrm{Cs}_{2} \mathrm{CO}_{3}$ (48.9 mg, $0.150 \mathrm{mmol}, 1.50$ equiv), $\mathrm{Cu}(\mathrm{MeCN})_{4} \mathrm{PF}_{6}(1.9 \mathrm{mg}, 5.0 \mathrm{~mol} \%)$ and $\mathbf{L 1}(2.0$ $\mathrm{mg}, 6.0 \mathrm{~mol} \%)$ in $\mathrm{CH}_{2} \mathrm{Cl}_{2}(2.0 \mathrm{~mL})$ at $0{ }^{\circ} \mathrm{C}$ in ethanol bath for $1 \mathrm{~h}$ afforded $\mathbf{3 j}$ (48.2 mg, 97\%, 94\% ee, yellow solid). $[\alpha]_{D}^{20}=+21\left(\mathrm{c}=1.03, \mathrm{CHCl}_{3}\right)$. HPLC conditions: Chiralpak AD-H, isopropanol/hexane $=10: 90$, flow: $1.0 \mathrm{~mL} / \mathrm{min}, \lambda=254 \mathrm{~nm} .{ }^{1} \mathbf{H}$ NMR $\left(400 \mathrm{MHz}, \mathrm{CDCl}_{3}\right) \delta$ $7.63(\mathrm{~d}, J=8.0 \mathrm{~Hz}, 1 \mathrm{H}), 7.29(\mathrm{~d}, J=8.0 \mathrm{~Hz}, 1 \mathrm{H}), 7.22(\mathrm{bs}, 1 \mathrm{H}), 6.86(\mathrm{~d}, J=8.0 \mathrm{~Hz}, 1 \mathrm{H}), 3.77$ (s, 3H), 3.71 (s, 3H), 2.39 (s, 3H), 2.24 (s, 3H), 1.92 (s, 6H). $\left.{ }^{13} \mathbf{C ~ N M R ~ ( 1 2 6 ~ M H z , ~} \mathrm{CDCl}_{3}\right) \delta$ 161.0, 158.4, 157.8, 141.8, 139.8, 139.6, 139.1, 137.9, 137.2, 136.8, 136.6, 135.8, 133.6, 133.0, 131.2, 130.8, 128.8, 121.6, 121.1, 100.0, 95.1, 53.0, 52.8, 20.8, 20.2, 18.7, 18.0, 16.4. HRMS (ESI) $\mathrm{m} / \mathrm{z}:[\mathrm{M}+\mathrm{Na}]^{+}$Calcd for $\mathrm{C}_{21} \mathrm{H}_{22} \mathrm{INO}_{5} \mathrm{Na}$ 518.0440; Found 518.0443.

Compound 3k was prepared following the Procedure A
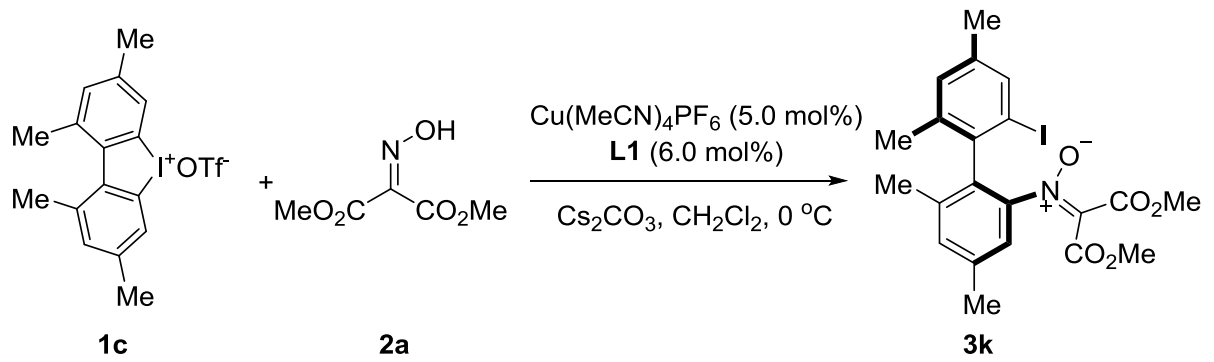

The reaction of $\mathbf{1 c}$ (48.4 mg, $0.100 \mathrm{mmol}, 1.00$ equiv), $2 \mathbf{a}(20.1 \mathrm{mg}, 0.125 \mathrm{mmol}, 1.25$ equiv), $\mathrm{Cs}_{2} \mathrm{CO}_{3}$ (48.9 mg, $0.150 \mathrm{mmol}, 1.50$ equiv), $\mathrm{Cu}(\mathrm{MeCN})_{4} \mathrm{PF}_{6}(1.9 \mathrm{mg}, 5.0 \mathrm{~mol} \%)$ and L1 (2.0 mg, $6.0 \mathrm{~mol} \%)$ in $\mathrm{CH}_{2} \mathrm{Cl}_{2}(2.0 \mathrm{~mL})$ at $0{ }^{\circ} \mathrm{C}$ in ethanol bath for $1 \mathrm{~h}$ afforded $3 \mathbf{k}(44.3$ mg, 89\%, 90\% ee, white solid). $[\alpha]_{D}^{20}=+34\left(\mathrm{c}=1.00, \mathrm{CHCl}_{3}\right)$. HPLC conditions: Chiralpak AD-H, isopropanol/hexane $=15: 85$, flow: $0.8 \mathrm{~mL} / \mathrm{min}, \lambda=254 \mathrm{~nm} .{ }^{1} \mathbf{H} \mathbf{N M R}(500 \mathrm{MHz}$, DMSO- $\left.d_{6}\right) \delta 7.56(\mathrm{~s}, 1 \mathrm{H}), 7.35(\mathrm{~s}, 1 \mathrm{H}), 7.25(\mathrm{~s}, 1 \mathrm{H}), 7.08(\mathrm{~s}, 1 \mathrm{H}), 3.70(\mathrm{~s}, 3 \mathrm{H}), 3.64(\mathrm{~s}, 3 \mathrm{H})$, 
2.37 (s, 3H), 2.25 (s, 3H), 1.90 (s, 3H), 1.89 (s, 3H). $\left.{ }^{13} \mathbf{C ~ N M R ~ ( 1 2 6 ~ M H z , ~} \mathrm{CDCl}_{3}\right) \delta 160.9$, $158.4,157.8,143.6,140.2,139.5,138.1,137.7,137.1,136.8,136.2,134.4,133.6,132.8$, 130.8, 122.6, 122.1, 103.1, 98.4, 53.1, 52.8, 21.7, 21.0, 20.5, 19.8. HRMS (ESI) m/z: [M + $\mathrm{Na}]^{+}$Calcd for $\mathrm{C}_{21} \mathrm{H}_{22} \mathrm{INO}_{5} \mathrm{Na}$ 518.0440; Found 518.0446.

Compound $\mathbf{3 1}$ was prepared following the Procedure A

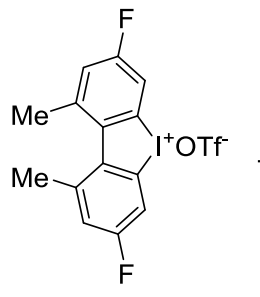

1d

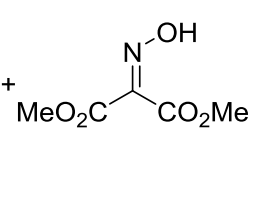

2a

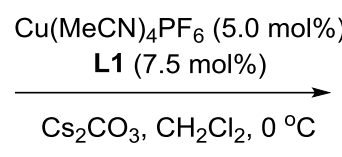

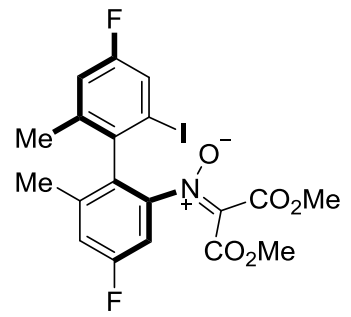

3!

The reaction of $1 \mathbf{d}$ (49.2 $\mathrm{mg}, 0.100 \mathrm{mmol}, 1.00$ equiv), $2 \mathbf{a}$ ( $20.1 \mathrm{mg}, 0.125 \mathrm{mmol}, 1.25$ equiv), $\mathrm{Cs}_{2} \mathrm{CO}_{3}$ (48.9 mg, $0.150 \mathrm{mmol}, 1.50$ equiv), $\mathrm{Cu}(\mathrm{MeCN})_{4} \mathrm{PF}_{6}(1.9 \mathrm{mg}, 5.0 \mathrm{~mol} \%)$ and $\mathbf{L 1}(2.5$ $\mathrm{mg}, 7.5 \mathrm{~mol} \%)(\mathrm{Cu} / \mathrm{L}=1: 1.5)$ in $\mathrm{CH}_{2} \mathrm{Cl}_{2}(2.0 \mathrm{~mL})$ at $0{ }^{\circ} \mathrm{C}$ in ethanol bath for $1 \mathrm{~h}$ afforded $3 \mathrm{I}$ (43.2 mg, 86\%, 99\% ee, yellow solid). $[\alpha]_{D}^{20}=+37\left(\mathrm{c}=0.98, \mathrm{CHCl}_{3}\right)$. HPLC conditions: Chiralpak OD-H, isopropanol/hexane $=1: 99$, flow: $1.0 \mathrm{~mL} / \mathrm{min}, \lambda=254 \mathrm{~nm} .{ }^{1} \mathbf{H}$ NMR $(400$ $\left.\mathrm{MHz}, \mathrm{CDCl}_{3}\right) \delta 7.48(\mathrm{dd}, J=8.0,2.4 \mathrm{~Hz}, 1 \mathrm{H}), 7.19(\mathrm{dd}, J=8.4,2.4 \mathrm{~Hz}, 1 \mathrm{H}), 7.08(\mathrm{bs}, 1 \mathrm{H})$, $6.97(\mathrm{dd}, J=8.4,2.4 \mathrm{~Hz}, 1 \mathrm{H}), 3.80(\mathrm{~s}, 3 \mathrm{H}), 3.74(\mathrm{~s}, 3 \mathrm{H}), 2.043(\mathrm{~s}, 3 \mathrm{H}), 2.036$ (s, 3H). ${ }^{19} \mathbf{F}$ NMR $\left(376 \mathrm{MHz}, \mathrm{CDCl}_{3}\right) \delta-111.83,-112.83,-113.15 .{ }^{13} \mathrm{C} \mathbf{~ N M R}\left(126 \mathrm{MHz}, \mathrm{CDCl}_{3}\right) \delta 161.7(\mathrm{~d}, J=$ $252.0 \mathrm{~Hz}), \delta 161.0(\mathrm{~d}, J=252.0 \mathrm{~Hz}), 160.4,157.7,144.3(\mathrm{~d}, J=10.1 \mathrm{~Hz}), 142.6,141.0,134.5$, 131.9, 123.5, 119.1 (d, $J=21.4 \mathrm{~Hz}), 117.0,110.2,103.0,97.6,53.3,53.1,22.0,21.0,20.0$. HRMS (ESI) m/z: $[\mathrm{M}+\mathrm{Na}]^{+}$Calcd for $\mathrm{C}_{19} \mathrm{H}_{16} \mathrm{~F}_{2} \mathrm{INO}_{5} \mathrm{Na}$ 525.9939; Found 525.9943.

Compound 3m was prepared following the Procedure A 
<smiles>[O+][o+]1c2cccc(Cl)c2c2c(Cl)cccc21</smiles>

$1 e$

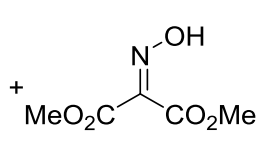

2a

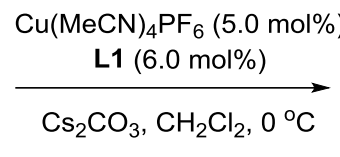

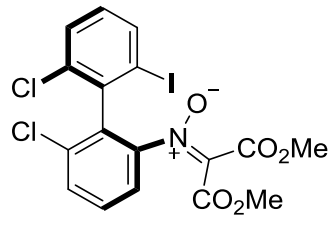

$3 m$

The reaction of $1 \mathbf{e}(49.7 \mathrm{mg}, 0.100 \mathrm{mmol}, 1.00$ equiv), $\mathbf{2 a}$ (20.1 mg, $0.125 \mathrm{mmol}, 1.25$ equiv), $\mathrm{Cs}_{2} \mathrm{CO}_{3}$ (48.9 mg, $0.150 \mathrm{mmol}, 1.50$ equiv), $\mathrm{Cu}(\mathrm{MeCN})_{4} \mathrm{PF}_{6}(1.9 \mathrm{mg}, 5.0 \mathrm{~mol} \%)$ and $\mathbf{L 1}(2.0$ $\mathrm{mg}, 6.0 \mathrm{~mol} \%)$ in $\mathrm{CH}_{2} \mathrm{Cl}_{2}(2.0 \mathrm{~mL})$ at $0{ }^{\circ} \mathrm{C}$ in ethanol bath for $1 \mathrm{~h}$ afforded $3 \mathrm{~m}(42.5 \mathrm{mg}, 84 \%$, 97\% ee, pale brown solid). $[\alpha]_{D}^{20}=+18\left(\mathrm{c}=1.38, \mathrm{CHCl}_{3}\right)$. HPLC conditions: Chiralpak IA, isopropanol/hexane $=30: 70$, flow: $1.0 \mathrm{~mL} / \mathrm{min}, \lambda=254 \mathrm{~nm} .{ }^{1} \mathbf{H}$ NMR $\left(400 \mathrm{MHz}, \mathrm{CDCl}_{3}\right) \delta$ $7.84(\mathrm{dd}, J=8.0,1.2 \mathrm{~Hz}, 1 \mathrm{H}), 7.68(\mathrm{dd}, J=8.0,1.6 \mathrm{~Hz}, 1 \mathrm{H}), 7.52(\mathrm{t}, J=8.0 \mathrm{~Hz}, 1 \mathrm{H}), 7.45(\mathrm{dt}$, $J=8.0,1.6 \mathrm{~Hz}, 2 \mathrm{H}), 7.05(\mathrm{t}, J=8.0 \mathrm{~Hz}, 1 \mathrm{H}), 3.79(\mathrm{~s}, 3 \mathrm{H}), 3.71(\mathrm{~s}, 3 \mathrm{H}) .{ }^{13} \mathbf{C} \mathbf{N M R}(101 \mathrm{MHz}$, $\left.\mathrm{CDCl}_{3}\right) \delta 160.3,157.5,144.1,137.6,136.9,135.3,134.9,134.0,132.5,131.4,131.1,129.3$, 129.0, 123.1, 102.4, 53.2, 52.9. HRMS (ESI) m/z: $[\mathrm{M}+\mathrm{Na}]^{+}$Calcd for $\mathrm{C}_{17} \mathrm{H}_{12} \mathrm{Cl}_{2} \mathrm{INO}_{5} \mathrm{Na}$ 529.9035; Found 529.9041.

Compound 3n was prepared following the Procedure A

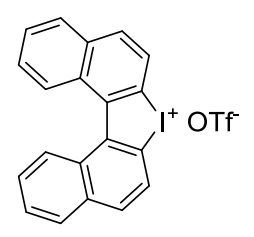

$1 f$

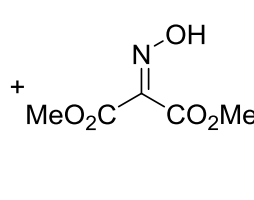

2a

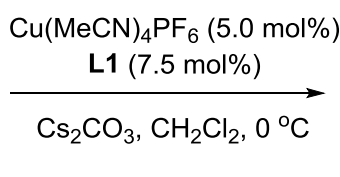

$\mathrm{Cs}_{2} \mathrm{CO}_{3}, \mathrm{CH}_{2} \mathrm{Cl}_{2}, 0^{\circ} \mathrm{C}$

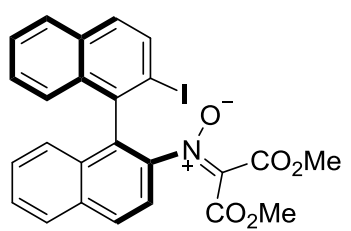

$3 n$

The reaction of $\mathbf{1 f}$ ( $52.8 \mathrm{mg}, 0.100 \mathrm{mmol}, 1.00$ equiv), $\mathbf{2 a}(20.1 \mathrm{mg}, 0.125 \mathrm{mmol}, 1.25$ equiv), $\mathrm{Cs}_{2} \mathrm{CO}_{3}$ (48.9 mg, $0.150 \mathrm{mmol}, 1.50$ equiv), $\mathrm{Cu}(\mathrm{MeCN})_{4} \mathrm{PF}_{6}(1.9 \mathrm{mg}, 5.0 \mathrm{~mol} \%)$ and $\mathbf{~ L 1}(2.5$ $\mathrm{mg}, 7.5 \mathrm{~mol} \%)(\mathrm{Cu} / \mathrm{L}=1: 1.5)$ in $\mathrm{CH}_{2} \mathrm{Cl}_{2}(2.0 \mathrm{~mL})$ at $0{ }^{\circ} \mathrm{C}$ in ethanol bath for $1 \mathrm{~h}$ afforded $3 \mathrm{n}$ (50.5 mg, 94\%, 93\% ee, yellow solid). $[\alpha]_{D}^{20}=-84\left(\mathrm{c}=0.78, \mathrm{CHCl}_{3}\right)$. HPLC conditions: Chiralpak IA, isopropanol/hexane $=30: 70$, flow: $1.0 \mathrm{~mL} / \mathrm{min}, \lambda=254 \mathrm{~nm} .{ }^{1} \mathbf{H}$ NMR $(400 \mathrm{MHz}$, $\left.\mathrm{CDCl}_{3}\right) \delta 8.12(\mathrm{~d}, J=8.8 \mathrm{~Hz}, 1 \mathrm{H}), 8.03(\mathrm{~d}, J=8.0,1 \mathrm{H}), 8.02(\mathrm{~d}, J=8.8,1 \mathrm{H}), 7.88(\mathrm{~d}, J=8.0$ 
$\mathrm{Hz}, 1 \mathrm{H}), 7.68(\mathrm{~d}, J=8.8 \mathrm{~Hz}, 1 \mathrm{H}), 7.60(\mathrm{t}, J=8.0,1 \mathrm{H}), 7.64-7.56(\mathrm{~m}, 1 \mathrm{H}), 7.49-7.37(\mathrm{~m}, 2 \mathrm{H})$, 7.32-7.08 (m, 3H), 3.64 (s, 3H), 3.51 (br, 3H). ${ }^{13} \mathbf{C}$ NMR (126 MHz, $\left.\mathrm{CDCl}_{3}\right) \delta 160.4,158.0$, $142.3,136.1,135.9,133.7,133.5,133.1,132.5,132.0,129.9,129.4,128.5,128.2,127.72$, 127.68, 126.9, 126.6, 126.2, 121.6, 101.4, 53.0, 52.5. HRMS (ESI) m/z: $[\mathrm{M}+\mathrm{Na}]^{+}$Calcd for $\mathrm{C}_{25} \mathrm{H}_{18} \mathrm{INO}_{5} \mathrm{Na}$ 562.0127; Found 562.0127.

Compound 30 was prepared following the Procedure A

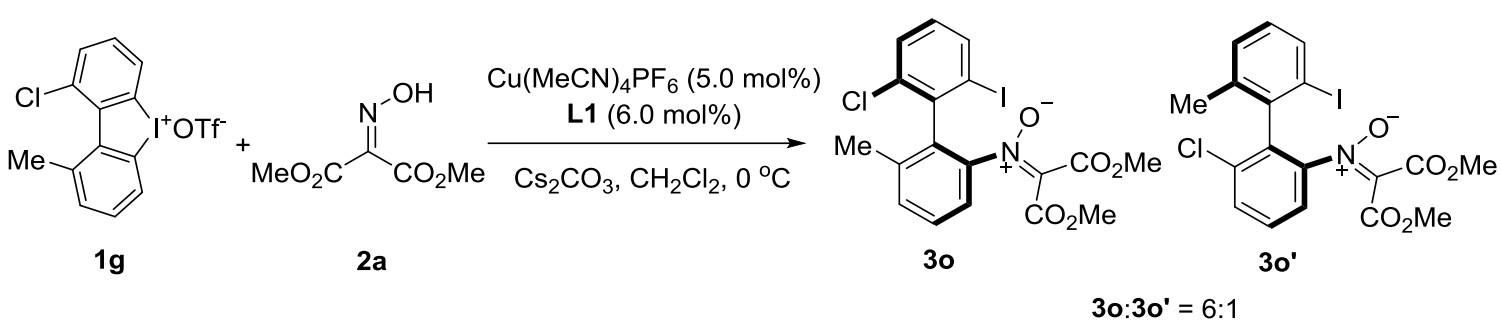

The reaction of $1 \mathrm{~g}$ (47.6 mg, $0.100 \mathrm{mmol}, 1.00$ equiv), $2 \mathrm{a}$ ( $20.1 \mathrm{mg}, 0.125 \mathrm{mmol}, 1.25$ equiv), $\mathrm{Cs}_{2} \mathrm{CO}_{3}$ (48.9 mg, $0.150 \mathrm{mmol}, 1.50$ equiv), $\mathrm{Cu}(\mathrm{MeCN})_{4} \mathrm{PF}_{6}(1.9 \mathrm{mg}, 5.0 \mathrm{~mol} \%)$ and $\mathbf{~ L 1}(2.0$ $\mathrm{mg}, 6.0 \mathrm{~mol} \%)$ in $\mathrm{CH}_{2} \mathrm{Cl}_{2}(2.0 \mathrm{~mL})$ at $0{ }^{\circ} \mathrm{C}$ in ethanol bath for $1 \mathrm{~h}$ afforded $\mathbf{3 0}: 3 \mathrm{o}^{\prime}=6: 1,(43.3$ mg, 89\%, 3o: 99\% ee; 3o': $94 \%$ ee, yellow solid). $[\alpha]_{D}^{20}=+23\left(\mathrm{c}=1.02, \mathrm{CHCl}_{3}\right)$. HPLC conditions: Chiralpak AD-H, isopropanol/hexane $=10: 90$, flow: $1.0 \mathrm{~mL} / \mathrm{min}, \lambda=254 \mathrm{~nm} .{ }^{1} \mathbf{H}$ NMR $\left(400 \mathrm{MHz}, \mathrm{CDCl}_{3}\right) \delta 7.83(\mathrm{dd}, J=8.0 \mathrm{~Hz}, 0.8 \mathrm{~Hz}, 1 \mathrm{H}), 7.50-7.43(\mathrm{~m}, 3 \mathrm{H}), 7.35(\mathrm{dd}, J=$ 6.8, 2.4 Hz, 1H), 7.02 (t, $J=8.0 \mathrm{~Hz}, 1 \mathrm{H}), 3.79$ (s, 3H), 3.69 (s, 3H), 2.10 (s, 3H). ${ }^{13} \mathbf{C}$ NMR $\left(126 \mathrm{MHz}, \mathrm{CDCl}_{3}\right) \delta 160.8,157.8,143.3,138.6,137.7,135.4,133.9,132.1,130.6,129.2,128.2$, 122.1, 103.0, 53.1, 52.8, 19.7. HRMS (ESI) m/z: $[\mathrm{M}+\mathrm{H}]^{+}$Calcd for $\mathrm{C}_{18} \mathrm{H}_{16} \mathrm{ClINO}_{5} 487.9762$; Found 487.9758 .

Compound 3p was prepared following the Procedure A 


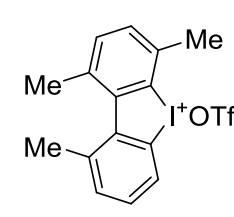

1h

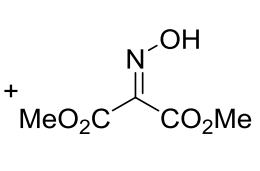

$2 \mathbf{a}$

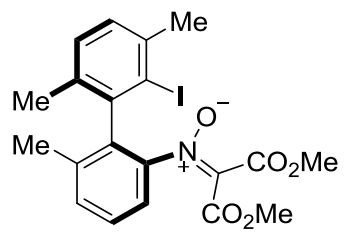

$3 p$

The reaction of $\mathbf{1 h}$ ( $47.0 \mathrm{mg}, 0.100 \mathrm{mmol}, 1.00$ equiv), $\mathbf{2 a}(20.1 \mathrm{mg}, 0.125 \mathrm{mmol}, 1.25$ equiv), $\mathrm{Cs}_{2} \mathrm{CO}_{3}$ (48.9 mg, $0.150 \mathrm{mmol}, 1.50$ equiv), $\mathrm{Cu}(\mathrm{MeCN})_{4} \mathrm{PF}_{6}(1.9 \mathrm{mg}, 5.0 \mathrm{~mol} \%)$ and $\mathbf{L 1}(2.0$ $\mathrm{mg}, 6.0 \mathrm{~mol} \%)$ in $\mathrm{CH}_{2} \mathrm{Cl}_{2}(2.0 \mathrm{~mL})$ at $0{ }^{\circ} \mathrm{C}$ in ethanol bath for $1 \mathrm{~h}$ afforded $3 p(44.7 \mathrm{mg}, 93 \%$, 94\% ee, brown solid). $[\alpha]_{D}^{20}=+57\left(\mathrm{c}=0.83, \mathrm{CHCl}_{3}\right)$. HPLC conditions: Chiralpak AD-H, isopropanol/hexane $=5: 95$, flow: $1.0 \mathrm{~mL} / \mathrm{min}, \lambda=254 \mathrm{~nm} .{ }^{1} \mathbf{H} \mathbf{~ N M R}\left(400 \mathrm{MHz}, \mathrm{CDCl}_{3}\right) \delta 7.45$ $(\mathrm{d}, J=7.2 \mathrm{~Hz}, 1 \mathrm{H}), 7.41(\mathrm{t}, J=7.6 \mathrm{~Hz}, 1 \mathrm{H}), 7.32(\mathrm{bs}, 1 \mathrm{H}), 7.14(\mathrm{~d}, J=8.0 \mathrm{~Hz}, 1 \mathrm{H}), 7.10(\mathrm{~d}, J$ $=7.6 \mathrm{~Hz}, 1 \mathrm{H}), 3.77(\mathrm{~s}, 3 \mathrm{H}), 3.70(\mathrm{~s}, 3 \mathrm{H}), 2.46(\mathrm{~s}, 3 \mathrm{H}), 2.02(\mathrm{~s}, 3 \mathrm{H}), 2.00(\mathrm{~s}, 3 \mathrm{H}) .{ }^{13} \mathbf{C}$ NMR $\left(101 \mathrm{MHz}, \mathrm{CDCl}_{3}\right) \delta 160.8,157.8,143.2,139.6,139.2,138.3,137.8,137.3,133.6,132.0,129.5$, 129.0, 127.3, 122.1, 109.7, 104.8, 53.0, 52.7, 29.3, 21.1, 20.3, 19.7. HRMS (ESI) m/z: [M + Na] ${ }^{+}$Calcd for $\mathrm{C}_{20} \mathrm{H}_{20} \mathrm{INO}_{5} \mathrm{Na}$ 504.0284; Found 504.0286.

Compound 3q was prepared following the Procedure A<smiles>Cc1c(Cl)cc(C)c2c1oc1cccc(C)c12</smiles>

$1 \mathrm{i}$

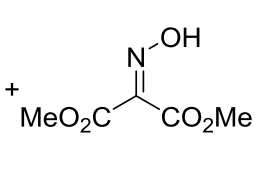

$2 \mathbf{a}$

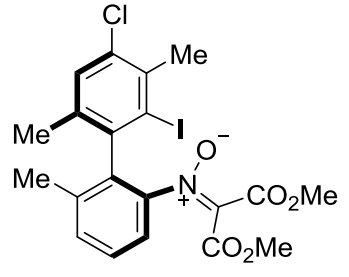

$3 q$

The reaction of $\mathbf{1 i}$ ( $50.5 \mathrm{mg}, 0.100 \mathrm{mmol}, 1.00$ equiv), $\mathbf{2 a}(20.1 \mathrm{mg}, 0.125 \mathrm{mmol}, 1.25$ equiv), $\mathrm{Cs}_{2} \mathrm{CO}_{3}$ (48.9 mg, $0.150 \mathrm{mmol}, 1.50$ equiv), $\mathrm{Cu}(\mathrm{MeCN})_{4} \mathrm{PF}_{6}(1.9 \mathrm{mg}, 5.0 \mathrm{~mol} \%)$ and $\mathbf{L 1}(2.0$ $\mathrm{mg}, 6.0 \mathrm{~mol} \%)$ in $\mathrm{CH}_{2} \mathrm{Cl}_{2}(2.0 \mathrm{~mL})$ at $0{ }^{\circ} \mathrm{C}$ in ethanol bath for $1 \mathrm{~h}$ afforded $3 \mathbf{q}(45.9 \mathrm{mg}, 89 \%$, 94\% ee, brown solid). $[\alpha]_{D}^{20}=+34\left(\mathrm{c}=1.37, \mathrm{CHCl}_{3}\right)$. HPLC conditions: Chiralpak AD-H, isopropanol/hexane $=10: 90$, flow: $1.0 \mathrm{~mL} / \mathrm{min}, \lambda=254 \mathrm{~nm} .{ }^{1} \mathbf{H}$ NMR $\left(400 \mathrm{MHz}, \mathrm{CDCl}_{3}\right) \delta$ $7.46(\mathrm{~d}, J=7.6 \mathrm{~Hz}, 1 \mathrm{H}), 7.41(\mathrm{t}, J=7.6 \mathrm{~Hz}, 1 \mathrm{H}), 7.31(\mathrm{br}, 1 \mathrm{H}), 7.25(\mathrm{~s}, 1 \mathrm{H}), 3.80(\mathrm{~s}, 3 \mathrm{H}), 3.71$ 
(s, 3H), 2.63 (s, 3H), 2.02 (s, 3H), 1.99 (s, 3H). $\left.{ }^{13} \mathbf{C ~ N M R ~ ( 1 2 6 ~ M H z , ~} \mathrm{CDCl}_{3}\right) \delta$ 160.7, 157.7, 143.0, 138.6, 137.3, 136.5, 135.0, 133.6, 132.6, 132.2, 130.4, 127.6, 122.1, 110.9, 105.7, 53.0, 52.8, 27.1, 21.0, 20.3, 19.7. HRMS (ESI) m/z: $[\mathrm{M}+\mathrm{Na}]^{+} \mathrm{Calcd}$ for $\mathrm{C}_{20} \mathrm{H}_{19} \mathrm{ClINO}_{5} \mathrm{Na}$ 537.9894; Found 537.9901.

Compound 3r was prepared following the Procedure A

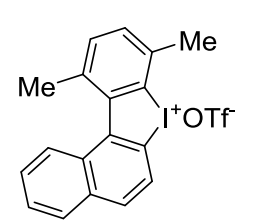

1j

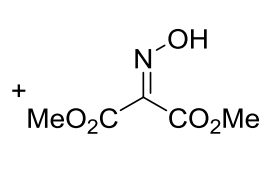

2a

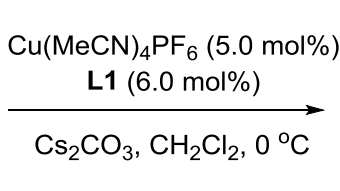

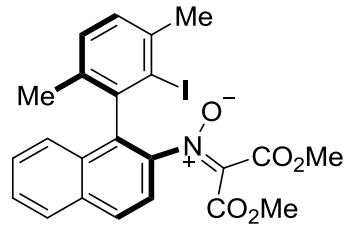

$3 r$

The reaction of $\mathbf{1 j}$ (50.6 mg, $0.100 \mathrm{mmol}, 1.00$ equiv), $\mathbf{2 a}(20.1 \mathrm{mg}, 0.125 \mathrm{mmol}, 1.25$ equiv), $\mathrm{Cs}_{2} \mathrm{CO}_{3}$ (48.9 mg, $0.150 \mathrm{mmol}, 1.50$ equiv), $\mathrm{Cu}(\mathrm{MeCN})_{4} \mathrm{PF}_{6}(1.9 \mathrm{mg}, 5.0 \mathrm{~mol} \%)$ and $\mathbf{L 1}(2.0$ $\mathrm{mg}, 6.0 \mathrm{~mol} \%)$ in $\mathrm{CH}_{2} \mathrm{Cl}_{2}(2.0 \mathrm{~mL})$ at $0{ }^{\circ} \mathrm{C}$ in ethanol bath for $1 \mathrm{~h}$ afforded $3 \mathbf{r}(52.2 \mathrm{mg},>99 \%$, 93\% ee, brown solid). $[\alpha]_{D}^{20}=+12\left(\mathrm{c}=1.00, \mathrm{CHCl}_{3}\right)$. HPLC conditions: Chiralpak IA, isopropanol/hexane $=30: 70$, flow: $1.0 \mathrm{~mL} / \mathrm{min}, \lambda=254 \mathrm{~nm} .{ }^{1} \mathbf{H}$ NMR $\left(400 \mathrm{MHz}, \mathrm{CDCl}_{3}\right) \delta$ $8.00(\mathrm{~d}, J=8.8 \mathrm{~Hz}, 1 \mathrm{H}), 7.97(\mathrm{~d}, J=8.0 \mathrm{~Hz}, 1 \mathrm{H}), 7.62-7.51(\mathrm{br}, 1 \mathrm{H}), 7.58(\mathrm{ddd}, J=8.4,7.2$, $1.2 \mathrm{~Hz}, 1 \mathrm{H}), 7.47$ (ddd, $J=8.4,7.2,1.2 \mathrm{~Hz}, 1 \mathrm{H}), 7.34$ (d, $J=8.4 \mathrm{~Hz}, 1 \mathrm{H}), 7.24(\mathrm{~d}, J=8.0 \mathrm{~Hz}$, 1H), $7.17(\mathrm{~d}, J=7.6 \mathrm{~Hz}, 1 \mathrm{H}), 3.81(\mathrm{~s}, 3 \mathrm{H}), 3.69$ (s, 3H), 2.50 (s, 3H), $1.92(\mathrm{~s}, 3 \mathrm{H}) .{ }^{13} \mathbf{C}$ NMR $\left(126 \mathrm{MHz}, \mathrm{CDCl}_{3}\right) \delta 160.8,157.9,140.7,139.3,138.6,135.7,135.1,134.9,133.8,131.5,129.5$, 128.4, 128.0, 127.6, 127.5, 126.5, 122.0, 110.1, 105.4, 53.2, 52.8, 29.3, 21.4, 20.5. HRMS (ESI) $\mathrm{m} / \mathrm{z}:[\mathrm{M}+\mathrm{Na}]^{+}$Calcd for $\mathrm{C}_{23} \mathrm{H}_{20} \mathrm{INO}_{5} \mathrm{Na}$ 540.0284; Found 540.0280.

Compound 3s was prepared following the Procedure A 


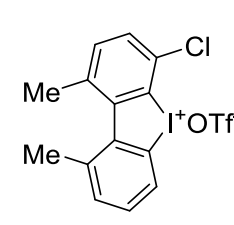

$1 \mathrm{k}$

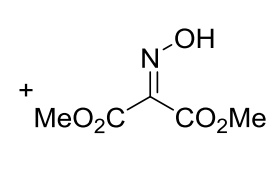

$2 \mathbf{a}$

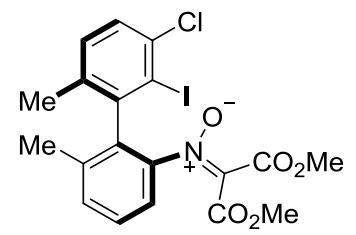

$3 s$

The reaction of $1 \mathbf{k}$ (49.1 mg, $0.100 \mathrm{mmol}, 1.00$ equiv), $2 \mathbf{a}(20.1 \mathrm{mg}, 0.125 \mathrm{mmol}, 1.25$ equiv), $\mathrm{Cs}_{2} \mathrm{CO}_{3}$ (48.9 mg, $0.150 \mathrm{mmol}, 1.50$ equiv), $\mathrm{Cu}(\mathrm{MeCN})_{4} \mathrm{PF}_{6}(1.9 \mathrm{mg}, 5.0 \mathrm{~mol} \%)$ and $\mathbf{L 1}(2.5$ $\mathrm{mg}, 7.5 \mathrm{~mol} \%)(\mathrm{Cu} / \mathrm{L}=1: 1.5)$ in $\mathrm{CH}_{2} \mathrm{Cl}_{2}(2.0 \mathrm{~mL})$ at $0{ }^{\circ} \mathrm{C}$ in ethanol bath for $1 \mathrm{~h}$ afforded $3 \mathrm{~s}$ (48.3 mg, 96\%, 76\% ee, pale brown solid). $[\alpha]_{D}^{20}=+50\left(\mathrm{c}=0.96, \mathrm{CHCl}_{3}\right)$. HPLC conditions: Chiralpak AD-H, isopropanol/hexane $=5: 95$, flow: $1.0 \mathrm{~mL} / \mathrm{min}, \lambda=254 \mathrm{~nm} .{ }^{1} \mathbf{H}$ NMR $(400$ $\left.\mathrm{MHz}, \mathrm{CDCl}_{3}\right) \delta 7.47(\mathrm{~d}, J=7.2 \mathrm{~Hz}, 1 \mathrm{H}), 7.42(\mathrm{t}, J=7.6 \mathrm{~Hz}, 1 \mathrm{H}), 7.36(\mathrm{~d}, J=8.0 \mathrm{~Hz}, 1 \mathrm{H}), 7.33$ (br, 1H), $7.17(\mathrm{~d}, J=8.0 \mathrm{~Hz}, 1 \mathrm{H}), 3.79(\mathrm{~s}, 3 \mathrm{H}), 3.71(\mathrm{~s}, 3 \mathrm{H}), 2.03(\mathrm{~s}, 3 \mathrm{H}), 2.02(\mathrm{~s}, 3 \mathrm{H}),{ }^{13} \mathrm{C}$ NMR $\left(126 \mathrm{MHz}, \mathrm{CDCl}_{3}\right) \delta 160.74,157.92,143.0,142.0,138.2,137.3,136.3,133.6,132.3$, $130.8,128.5,127.8,122.2,107.1,102.1,53.2,52.9,21.2,19.7$. HRMS $(E S I) \mathrm{m} / \mathrm{z}:[\mathrm{M}+\mathrm{Na}]^{+}$ Calcd for $\mathrm{C}_{19} \mathrm{H}_{17} \mathrm{ClINO}_{5} \mathrm{Na}$ 523.9738; Found 523.9752.

Compound 3t was prepared following the Procedure A

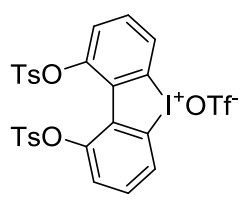

11

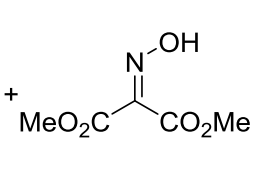

$2 \mathrm{a}$

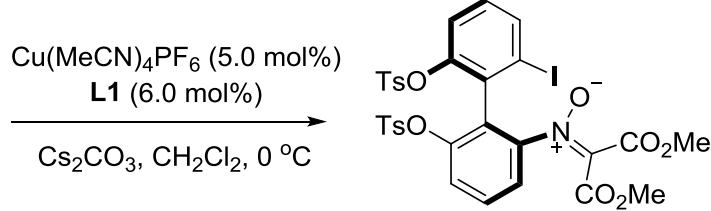

3t

The reaction of 11 (76.8 mg, $0.100 \mathrm{mmol}, 1.00$ equiv), $\mathbf{2 a}(20.1 \mathrm{mg}, 0.125 \mathrm{mmol}, 1.25$ equiv), $\mathrm{Cs}_{2} \mathrm{CO}_{3}$ (48.9 mg, $0.150 \mathrm{mmol}, 1.50$ equiv), $\mathrm{Cu}(\mathrm{MeCN})_{4} \mathrm{PF}_{6}(1.9 \mathrm{mg}, 5.0 \mathrm{~mol} \%)$ and $\mathbf{L 1}(2.0$ $\mathrm{mg}, 6.0 \mathrm{~mol} \%)$ in $\mathrm{CH}_{2} \mathrm{Cl}_{2}(2.0 \mathrm{~mL})$ at $0{ }^{\circ} \mathrm{C}$ in ethanol bath for $1 \mathrm{~h}$ afforded $3 \mathrm{t}(65.5 \mathrm{mg}, 84 \%$, 99\% ee, white solid). $[\alpha]_{D}^{20}=-12\left(\mathrm{c}=0.59, \mathrm{CHCl}_{3}\right)$. HPLC conditions: Chiralpak AD-H, isopropanol/hexane $=30: 70$, flow: $1.0 \mathrm{~mL} / \mathrm{min}, \lambda=254 \mathrm{~nm} .{ }^{1} \mathbf{H}$ NMR $\left(400 \mathrm{MHz}, \mathrm{CDCl}_{3}\right) \delta$ $7.63(\mathrm{~d}, J=8.0 \mathrm{~Hz}, 3 \mathrm{H}), 7.58-7.45(\mathrm{~m}, 4 \mathrm{H}), 7.41(\mathrm{~d}, J=8.4 \mathrm{~Hz}, 1 \mathrm{H}), 7.31(\mathrm{~d}, J=7.6 \mathrm{~Hz}, 1 \mathrm{H})$, 
$7.22(\mathrm{~d}, J=8.0 \mathrm{~Hz}, 2 \mathrm{H}), 7.18(\mathrm{~d}, J=8.0 \mathrm{~Hz}, 2 \mathrm{H}), 7.01(\mathrm{t}, J=8.0 \mathrm{~Hz}, 1 \mathrm{H}), 3.77(\mathrm{~s}, 6 \mathrm{H}), 2.45(\mathrm{~s}$, 3H), 2.41 (s, 3H). ${ }^{13} \mathbf{C}$ NMR (126 MHz, $\left.\mathrm{CDCl}_{3}\right) \delta 160.5,157.3,148.3,147.6,145.5,145.30$, $145.27,145.1,143.8,136.9,134.1,133.24,133.22,132.7,130.95,130.92,129.63,129.59$, 129.58, 129.4, 128.6, 128.1, 127.9, 125.3, 121.5, 121.3, 102.1, 53.2, 53.1, 21.7, 21.6. HRMS (ESI) $\mathrm{m} / \mathrm{z}:[\mathrm{M}+\mathrm{Na}]^{+}$Calcd for $\mathrm{C}_{31} \mathrm{H}_{26} \mathrm{NNO}_{11} \mathrm{~S}_{2} \mathrm{Na}$ 801.9890; Found 801.9898.

Synthesis of Compound $\mathbf{4 t}$

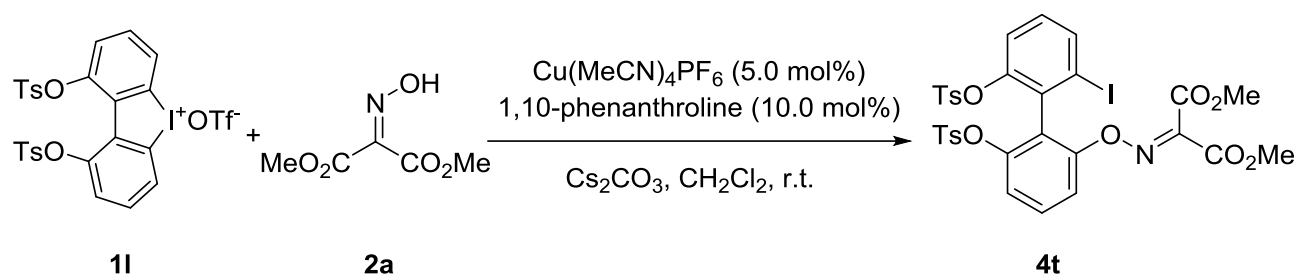

To a Schlenk tube was added 11 ( $76.8 \mathrm{mg}, 0.100 \mathrm{mmol}, 1.00$ equiv), $\mathbf{2 a}(20.1 \mathrm{mg}, 0.125 \mathrm{mmol}$, 1.25 equiv), $\mathrm{Cs}_{2} \mathrm{CO}_{3}$ (48.9 mg, $0.150 \mathrm{mmol}, 1.50$ equiv), $\mathrm{Cu}(\mathrm{MeCN})_{4} \mathrm{PF}_{6}(1.9 \mathrm{mg}, 5.0 \mathrm{~mol} \%)$ and o-phenanthroline $(1.8 \mathrm{mg}, 10.0 \mathrm{~mol} \%)$ followed by $\mathrm{CH}_{2} \mathrm{Cl}_{2}(2.0 \mathrm{~mL})$ under nitrogen at room temperature. After being stirred for $72 \mathrm{~h}$ (monitored by TLC), the solvent was removed and the residue was purified by flash column chromatography on silica gel to afford the desired product $4 \mathbf{t}$ (40.6 mg, 52\%, colorless oil). ${ }^{1} \mathbf{H} \mathbf{~ N M R}\left(400 \mathrm{MHz}, \mathrm{CDCl}_{3}\right) \delta 7.71(\mathrm{~d}, J=8.0 \mathrm{~Hz}$, $1 \mathrm{H}), 7.48(\mathrm{~d}, J=8.0 \mathrm{~Hz}, 2 \mathrm{H}), 7.36-7.28(\mathrm{~m}, 4 \mathrm{H}), 7.22(\mathrm{~d}, J=8.4 \mathrm{~Hz}, 1 \mathrm{H}), 7.17-7.13(\mathrm{~m}, 3 \mathrm{H})$, 7.07-7.03 (m, 3H), 3.92 (s, 3H), 3.48 (s, 3H), 2.39 (s, 3H), 2.36 (s, 3H). ${ }^{13}$ C NMR (126 MHz, $\left.\mathrm{CDCl}_{3}\right) \delta 159.5,159.3,155.2,147.2,147.1,145.7,145.0,144.8,137.0,133.0,132.6,130.4$, $130.2,130.1,129.62,129.55,127.8,127.5,122.7,119.6,117.0,112.1,102.0,53.4,52.6,21.6$, 21.5. HRMS (ESI) m/z: $[\mathrm{M}+\mathrm{H}]^{+}$Calcd for $\mathrm{C}_{31} \mathrm{H}_{27} \mathrm{INO}_{11} \mathrm{~S}_{2}$ 780.0070; Found 780.0070.

Compound 3u was prepared following the Procedure A 


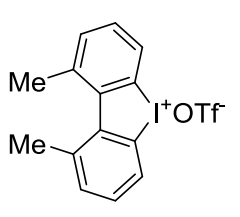

$1 a$

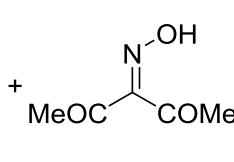

2j

j

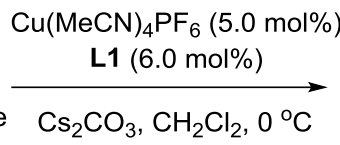

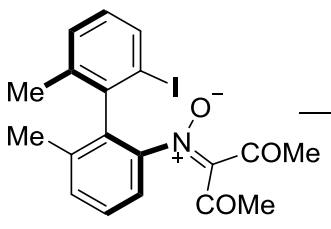

$3 u$

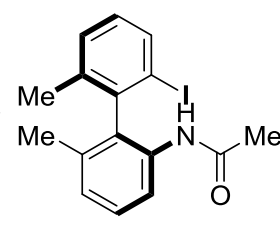

$3 u^{\prime}$

The reaction of $\mathbf{1 a}$ ( $45.6 \mathrm{mg}, 0.100 \mathrm{mmol}, 1.00$ equiv), $\mathbf{2 j}$ ( $16.1 \mathrm{mg}, 0.125 \mathrm{mmol}, 1.25$ equiv), $\mathrm{Cs}_{2} \mathrm{CO}_{3}$ (48.9 mg, $0.150 \mathrm{mmol}, 1.50$ equiv), $\mathrm{Cu}(\mathrm{MeCN})_{4} \mathrm{PF}_{6}(1.9 \mathrm{mg}, 5.0 \mathrm{~mol} \%)$ and $\mathbf{~ L 1}(2.0$ $\mathrm{mg}, 6.0 \mathrm{~mol} \%)$ in $\mathrm{CH}_{2} \mathrm{Cl}_{2}(2.0 \mathrm{~mL})$ at $0{ }^{\circ} \mathrm{C}$ in ethanol bath for $4 \mathrm{~h}$ afforded $3 \mathbf{u}(30.9 \mathrm{mg}, 71 \%$, yellow oil), which partially degraded to $\mathbf{3} \mathbf{u}$ ' during NMR analysis. Compound $\mathbf{3} \mathbf{u}$ ' was isolated in $73 \%$ yield $(19.0 \mathrm{mg}, 86 \%$ ee, yellow solid) upon it standing at room temperature for 10 days in $\mathrm{CDCl}_{3}$.

3u: HPLC conditions: Chiralpak AD-H, isopropanol/hexane $=5: 95$, flow: $1.0 \mathrm{~mL} / \mathrm{min}, \lambda=254$ nm. ${ }^{1} \mathbf{H}$ NMR $\left(500 \mathrm{MHz}, \mathrm{CDCl}_{3}\right) \delta 7.77(\mathrm{~d}, J=8.0 \mathrm{~Hz}, 1 \mathrm{H}), 7.44(\mathrm{~d}, J=4.6 \mathrm{~Hz}, 2 \mathrm{H}), 7.32(\mathrm{~s}$, 1H), $7.25(\mathrm{~d}, J=8.5 \mathrm{~Hz}, 1 \mathrm{H}), 6.99(\mathrm{t}, J=8.0 \mathrm{~Hz}, 1 \mathrm{H}), 2.15(\mathrm{~s}, 3 \mathrm{H}), 2.06(\mathrm{~s}, 3 \mathrm{H}), 2.05(\mathrm{~s}, 3 \mathrm{H})$, $1.93(\mathrm{~s}, 3 \mathrm{H}) .{ }^{13} \mathbf{C}$ NMR $\left(126 \mathrm{MHz}, \mathrm{CDCl}_{3}\right) \delta 196.7,145.2,139.5,138.2,136.6,135.9,131.8$, 130.0, 129.9, 128.0, 122.7, 30.5, 27.5, 21.5, 19.9. HRMS (ESI) $\mathrm{m} / \mathrm{z}:[\mathrm{M}+\mathrm{Na}]^{+}$Calcd for $\mathrm{C}_{19} \mathrm{H}_{18} \mathrm{INO}_{3} \mathrm{Na}$ 458.0229; Found 458.0226.

3u': HPLC conditions: Chiralpak AD-H, isopropanol/hexane $=2.5: 97.5$, flow: $1.0 \mathrm{~mL} / \mathrm{min}, \lambda$ $=254 \mathrm{~nm} .{ }^{1} \mathbf{H}$ NMR $\left(400 \mathrm{MHz}, \mathrm{CDCl}_{3}\right) \delta 8.15(\mathrm{~d}, J=8.0 \mathrm{~Hz}, 1 \mathrm{H}), 7.86(\mathrm{~d}, J=8.0 \mathrm{~Hz}, 1 \mathrm{H})$, $7.34(\mathrm{~m}, 2 \mathrm{H}), 7.08(\mathrm{~d}, J=7.6 \mathrm{~Hz}, 1 \mathrm{H}), 7.05(\mathrm{t}, J=7.6 \mathrm{~Hz}, 1 \mathrm{H}), 6.44(\mathrm{~s}, 1 \mathrm{H}), 2.01(\mathrm{~s}, 3 \mathrm{H}), 1.92$ $(\mathrm{s}, 6 \mathrm{H}) .{ }^{13} \mathrm{C}$ NMR $\left(126 \mathrm{MHz}, \mathrm{CDCl}_{3}\right) \delta 168.1,140.7,139.0,137.4,135.9,134.5,133.3,130.6$, 130.0, 128.6, 126.0, 118.8, 101.3, 24.7, 21.2, 19.8. HRMS (ESI) m/z: $[\mathrm{M}+\mathrm{Na}]^{+}$Calcd for $\mathrm{C}_{16} \mathrm{H}_{16} \mathrm{INONa}$ 388.0174; Found 388.0173.

Synthesis of Compound $7 \mathbf{a}$ 


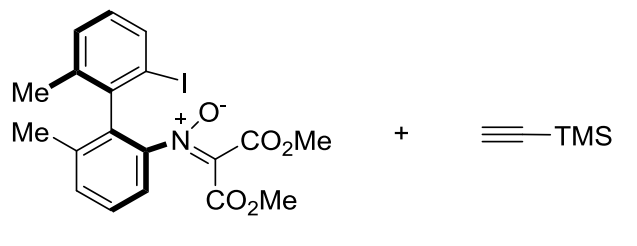

3a, $97 \%$ ee

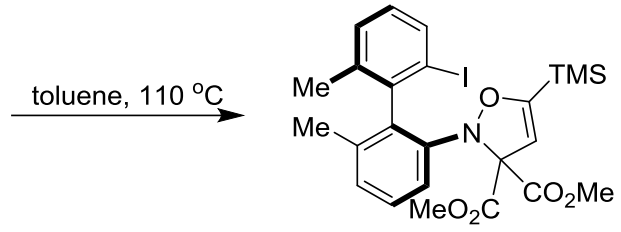

$7 a, 97 \%$ ee

To a $25 \mathrm{~mL}$ Schlenk tube containing $3 \mathbf{a}$ ( $46.7 \mathrm{mg}, 0.10 \mathrm{mmol}, 1.00$ equiv, $97 \%$ ee) and toluene $(2.0 \mathrm{~mL})$ was added trimethylsilylacetylene $(19.6 \mathrm{mg}, 0.20 \mathrm{mmol}, 2.00$ equiv) under nitrogen atmosphere, the flask was capped with a screw cap and stirred at $110{ }^{\circ} \mathrm{C}$ in oil bath for $5 \mathrm{~h}$. After complete consumption of starting material, the mixture was cooled to room temperature and then toluene was removed under vacuum, the residue was purified by flash column chromatography on silica gel (hexane/ethyl acetate $=20: 1$ ) to afford the desired compound $7 \mathbf{a}$ (41.8 mg, 74\%, 97\% ee, colorless oil). $[\alpha]_{D}^{20}=+227\left(\mathrm{c}=1.29, \mathrm{CHCl}_{3}\right)$. HPLC conditions: Chiralpak $(\mathrm{OD}+\mathrm{AD})$, isopropanol/hexane $=0.5: 99.5$, flow: $0.8 \mathrm{~mL} / \mathrm{min}, \lambda=254 \mathrm{~nm} .{ }^{1} \mathbf{H}$ NMR $\left(500 \mathrm{MHz}, \mathrm{CDCl}_{3}\right) \delta 7.78(\mathrm{~d}, J=8.0 \mathrm{~Hz}, 1 \mathrm{H}), 7.22(\mathrm{~d}, J=7.5 \mathrm{~Hz}, 1 \mathrm{H}), 7.18(\mathrm{t}, J=8.0 \mathrm{~Hz}, 1 \mathrm{H})$, $7.08(\mathrm{~d}, J=8.0 \mathrm{~Hz}, 1 \mathrm{H}), 6.97-6.94(\mathrm{~m}, 2 \mathrm{H}), 5.57(\mathrm{~s}, 1 \mathrm{H}), 3.78(\mathrm{~s}, 3 \mathrm{H}), 3.64(\mathrm{~s}, 3 \mathrm{H}), 2.07(\mathrm{~s}$, 3H), 1.88 (s, 3H), -0.02 (s, 9H). ${ }^{13} \mathbf{C}$ NMR (126 MHz, $\left.\mathrm{CDCl}_{3}\right) \delta 168.7,166.4,143.1,141.2$, $139.7,138.5,137.3,136.5,135.1,129.8,129.2,128.1,125.0,123.3,119.4,104.2,98.6,53.0$, 52.9, 21.2, 20.4, -2.5. HRMS (ESI) m/z: $[\mathrm{M}+\mathrm{H}]^{+}$Calcd for $\mathrm{C}_{24} \mathrm{H}_{28} \mathrm{INO}_{5} \mathrm{Si}$ 566.0860; Found 566.0852.

Synthesis of Compound 8a

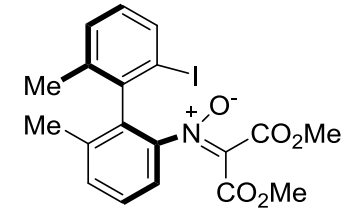

3a, $97 \%$ ee

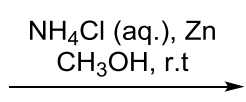

$\mathrm{CH}_{3} \mathrm{OH}$, r.t

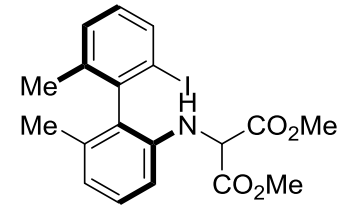

8 a, $97 \%$ ee

To a $25 \mathrm{~mL}$ Schlenk tube containing 3a (46.7 mg, 0.10 mmol, 1.00 equiv, $97 \%$ ee), $\mathrm{MeOH}$ $(1.0 \mathrm{~mL})$ and saturated aqueous $\mathrm{NH}_{4} \mathrm{Cl}(1.0 \mathrm{~mL})$ was added zinc powder $(65.4 \mathrm{mg}, 1.00 \mathrm{mmol}$, 10.00 equiv) in batches under nitrogen atmosphere, then the mixture was stirred at room 
temperature for $3 \mathrm{~h}$ until disappearance of starting material confirmed by TLC. The mixture was diluted with $\mathrm{H}_{2} \mathrm{O}$, extracted with $\mathrm{CH}_{2} \mathrm{Cl}_{2}(5.0 \mathrm{~mL} \times 2)$, washed with saturated aqueous $\mathrm{NaHCO}_{3}$ and brine. The collected organic layer was dried over anhydrous sodium sulfate. The solid $\mathrm{Na}_{2} \mathrm{SO}_{4}$ was filtered off, the filtrate was concentrated. The residue was purified by flash column chromatography on silica gel (hexanes/ethyl acetate $=10: 1$ ) to afford compound $\mathbf{8 a}$ (40.3 mg, 89\%, 97\% ee, yellow solid). $[\alpha]_{D}^{20}=+42\left(\mathrm{c}=1.35, \mathrm{CHCl}_{3}\right)$. HPLC conditions: Chiralpak AD-H, isopropanol $/$ hexane $=0.5: 99.5$, flow: $1.0 \mathrm{~mL} / \mathrm{min}, \lambda=254 \mathrm{~nm} .{ }^{1} \mathbf{H}$ NMR $(400$ $\left.\mathrm{MHz}, \mathrm{CDCl}_{3}\right) \delta 7.85(\mathrm{~d}, J=7.6 \mathrm{~Hz}, 1 \mathrm{H}), 7.33(\mathrm{~d}, J=7.6 \mathrm{~Hz}, 1 \mathrm{H}), 7.19(\mathrm{t}, J=8.0 \mathrm{~Hz}, 1 \mathrm{H}), 7.02$ $(\mathrm{t}, J=7.6 \mathrm{~Hz}, 1 \mathrm{H}), 6.76(\mathrm{~d}, J=8.0 \mathrm{~Hz}, 1 \mathrm{H}), 6.46(\mathrm{~d}, J=8.0 \mathrm{~Hz}, 1 \mathrm{H}), 4.78(\mathrm{~d}, J=7.2 \mathrm{~Hz}, 1 \mathrm{H})$, $4.26(\mathrm{~d}, J=7.2 \mathrm{~Hz}, 1 \mathrm{H}), 3.75$ (s, 6H), 2.07 (s, 3H), 1.90 (s, 3H). $\left.{ }^{13} \mathbf{C ~ N M R ~ ( 1 0 1 ~ M H z , ~} \mathrm{CDCl}_{3}\right)$ $\delta 168.3,167.7,141.8,140.9,139.3,137.3,136.5,130.5,130.1,129.7,128.8,120.4,108.2$, 102.4, 60.4, 53.2, 53.1, 21.0, 19.8. HRMS (ESI) $\mathrm{m} / \mathrm{z}$ : $[\mathrm{M}+\mathrm{Na}]^{+}$Calcd for $\mathrm{C}_{19} \mathrm{H}_{20} \mathrm{INO}_{4} \mathrm{Na}$ 476.0335; Found 476.0337

\section{Synthesis of Compound 9a}

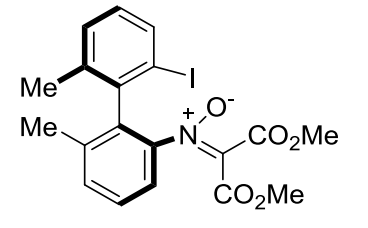

$3 a, 97 \%$ ee

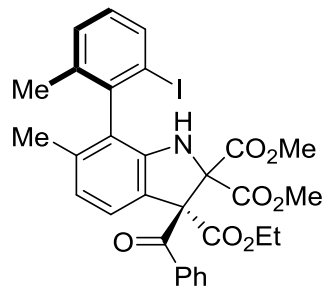

9a, $97 \%$ ee

To a $25 \mathrm{~mL}$ Schlenk tube containing 3a (140.2 mg, $0.30 \mathrm{mmol}, 1.00$ equiv, $97 \%$ ee) and toluene $(6.0 \mathrm{~mL})$ was added ethyl phenylpropiolate $(104.5 \mathrm{mg}, 0.60 \mathrm{mmol}, 2.00$ equiv) dropwise under nitrogen atmosphere, the flask was capped with a screw cap and then stirred at $110{ }^{\circ} \mathrm{C}$ in oil bath for $24 \mathrm{~h}$ until disappearance of starting material confirmed by TLC. Then mixture was cooled to room temperature and toluene was removed under vacuum, the residue was purified by flash column chromatography on silica gel (hexane/ethyl acetate $=5: 1$ ) to afford 9a [86.0 mg, 45\%, dr = 6:1, 97\% ee (major isomer), pale yellow solid]. $[\alpha]_{D}^{20}=+336$ 
$\left(\mathrm{c}=0.64, \mathrm{CHCl}_{3}\right) . \mathrm{HPLC}$ conditions: Chiralpak AD-H, isopropanol/hexane $=10: 90$, flow: 1.0 $\mathrm{mL} / \mathrm{min}, \lambda=254 \mathrm{~nm} .{ }^{1} \mathbf{H}$ NMR $\left(500 \mathrm{MHz}, \mathrm{CDCl}_{3}\right) \delta 7.81(\mathrm{~d}, J=8.0 \mathrm{~Hz}, 1 \mathrm{H}), 7.68(\mathrm{~d}, J=8.0$ $\mathrm{Hz}, 2 \mathrm{H}), 7.45$ (t, $J=7.5 \mathrm{~Hz}, 1 \mathrm{H}), 7.32-7.28(\mathrm{~m}, 3 \mathrm{H}), 7.25(\mathrm{~m}, 1 \mathrm{H}), 7.01(\mathrm{t}, J=8.0 \mathrm{~Hz}, 1 \mathrm{H})$, $6.73(\mathrm{~d}, J=8.0 \mathrm{~Hz}, 1 \mathrm{H}), 4.50(\mathrm{~s}, 1 \mathrm{H}), 4.28(\mathrm{q}, J=7.0 \mathrm{~Hz}, 2 \mathrm{H}), 3.70(\mathrm{~s}, 3 \mathrm{H}), 3.52(\mathrm{~s}, 3 \mathrm{H}), 2.14$ (s, 3H), $1.92(\mathrm{~s}, 3 \mathrm{H}), 1.27(\mathrm{t}, J=7.0 \mathrm{~Hz}, 3 \mathrm{H}) .{ }^{13} \mathbf{C} \mathbf{~ N M R}\left(126 \mathrm{MHz}, \mathrm{CDCl}_{3}\right) \delta$ 193.9, 168.8, $168.2,146.1,140.8,138.8,137.8,137.1,136.1,132.3,130.3,129.7,128.7,128.0,126.9,126.2$, 122.5, 121.6, 101.3, 78.7, 75.5, 62.2, 53.3, 52.8, 21.1, 19.2, 13.8. HRMS (ESI) m/z: $[\mathrm{M}+\mathrm{Na}]^{+}$ Calcd for $\mathrm{C}_{30} \mathrm{H}_{28} \mathrm{INO}_{7} \mathrm{Na}$ 664.0808; Found 664.0809.

Synthesis of Compound 10a

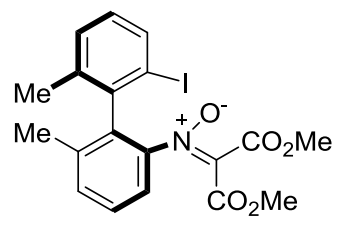

$3 a$
$4 \mathrm{M} \mathrm{HCl}$, dioxane, $0^{\circ} \mathrm{C}$ to r.t.

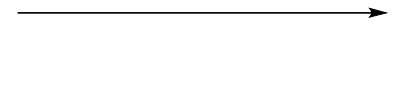

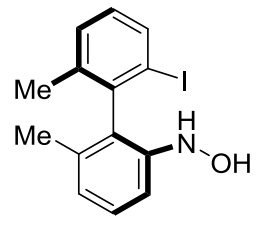

$10 \mathrm{a}$

To a $50 \mathrm{~mL}$ flask containing 3a (467.3 mg, $1.00 \mathrm{mmol}, 1.00$ equiv) was added 1,4-dioxane $(10.0 \mathrm{~mL})$ and $4 \mathrm{M}$ hydrochloric acid $(1.0 \mathrm{~mL}, 4.00 \mathrm{mmol}, 4.00$ equiv) under nitrogen atmosphere at $0{ }^{\circ} \mathrm{C}$ in ethanol bath. The reaction mixture was degassed with $\mathrm{N}_{2}$ for $5 \mathrm{~min}$, and stirred for another $3 \mathrm{~h}$ at room temperature until disappearance of starting material confirmed by TLC. The mixture was diluted with $\mathrm{H}_{2} \mathrm{O}$, extracted with $\mathrm{CH}_{2} \mathrm{Cl}_{2}(30.0 \mathrm{~mL}$ x2), washed with brine then dried over anhydrous sodium sulfate. After filtration, the solvent was removed by evaporation and the residue was purified by flash column chromatography on silica gel (hexanes/ethyl acetate $=20: 1)$ to afford 10a $(308.3 \mathrm{mg}, 91 \%$, brown-yellow oil). The ee value of this product was not determined due to its metastability and it would gradually decompose upon chiral HPLC analysis. However, the subsequent synthetic applications confirmed the high enantiopurity of 10a. $[\alpha]_{D}^{20}=+36\left(\mathrm{c}=3.32, \mathrm{CHCl}_{3}\right) .{ }^{1} \mathbf{H} \mathbf{N M R}\left(500 \mathrm{MHz}, \mathbf{C}_{\mathbf{6}} \mathbf{D}_{\mathbf{6}}\right) \delta 7.63(\mathrm{~d}, J$ $=8.0 \mathrm{~Hz}, 1 \mathrm{H}), 7.32(\mathrm{~d}, J=8.0 \mathrm{~Hz}, 1 \mathrm{H}), 7.25(\mathrm{t}, J=8.0 \mathrm{~Hz}, 1 \mathrm{H}), 6.86(\mathrm{~m}, 2 \mathrm{H}), 6.55(\mathrm{t}, J=8.0$ Hz, 1H), 6.09 (s, 1H), 5.02 (s, 1H), 1.85 (s, 6H). $\left.{ }^{13} \mathbf{C ~ N M R ~ ( 1 2 6 ~ M H z , ~} \mathbf{C}_{6} \mathbf{D}_{6}\right) \delta 146.7,141.6$, 
138.9, 137.6, 135.6, 130.5, 129.74, 129.71, 129.1, 123.8, 113.2, 101.9, 21.1, 19.4. HRMS (ESI)

m/z: $[\mathrm{M}+\mathrm{H}]^{+}$Calcd for $\mathrm{C}_{14} \mathrm{H}_{15} \mathrm{INO} 340.0198$; Found 340.0201 .

Synthesis of Compound 11a

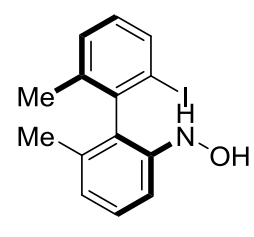

$10 \mathrm{a}$
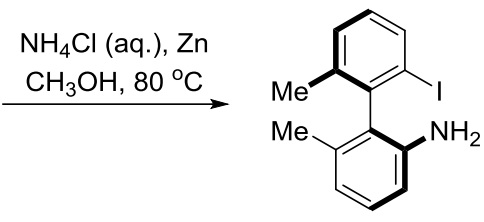

$11 \mathrm{a}$

To a $25 \mathrm{~mL}$ Schlenk tube containing 10a (169.6 mg, $0.50 \mathrm{mmol}, 1.00$ equiv), $\mathrm{MeOH}$ (5.0 $\mathrm{mL})$ and saturated aqueous $\mathrm{NH}_{4} \mathrm{Cl}$ solution $(5.0 \mathrm{~mL})$ was added zinc powder in batches (163.5 $\mathrm{mg}, 2.50 \mathrm{mmol}, 5.00$ equiv) under nitrogen atmosphere. The mixture was stirred at $80{ }^{\circ} \mathrm{C}$ in oil bath for $3 \mathrm{~h}$ then cooled to room temperature. The mixture was diluted with $\mathrm{H}_{2} \mathrm{O}$, extracted with $\mathrm{CH}_{2} \mathrm{Cl}_{2}(10.0 \mathrm{~mL} \times 2)$, washed with saturated aqueous $\mathrm{NaHCO}_{3}$ then brine. The collected organic layer was dried over anhydrous sodium sulfate. After filtration, the solvent was removed by evaporation and the residue was purified by flash column chromatography on silica gel (hexanes/ethyl acetate $=20: 1)$ to afford 11a $\left(153.3 \mathrm{mg}, 95 \%, 96 \%\right.$ ee, brown oil). $[\alpha]_{D}^{20}=$ $+33\left(\mathrm{c}=0.73, \mathrm{CHCl}_{3}\right) . \mathrm{HPLC}$ conditions: Chiralpak OD-H, isopropanol/hexane $=0.5: 99.5$, flow: $1.0 \mathrm{~mL} / \mathrm{min}, \lambda=254 \mathrm{~nm} .{ }^{1} \mathbf{H}$ NMR $\left(400 \mathrm{MHz}, \mathrm{CDCl}_{3}\right) \delta 7.83(\mathrm{~d}, J=8.0 \mathrm{~Hz}, 1 \mathrm{H}), 7.30$ $(\mathrm{d}, J=7.6 \mathrm{~Hz}, 1 \mathrm{H}), 7.13(\mathrm{t}, J=7.6 \mathrm{~Hz}, 1 \mathrm{H}), 6.98(\mathrm{t}, J=8.0 \mathrm{~Hz}, 1 \mathrm{H}), 6.73(\mathrm{~d}, J=7.6 \mathrm{~Hz}, 1 \mathrm{H})$, $6.65(\mathrm{~d}, J=8.0 \mathrm{~Hz}, 1 \mathrm{H}), 3.31(\mathrm{~s}, 2 \mathrm{H}), 2.09(\mathrm{~s}, 3 \mathrm{H}), 1.89(\mathrm{~s}, 3 \mathrm{H}) .{ }^{13} \mathbf{C ~ N M R}\left(126 \mathrm{MHz}, \mathrm{CDCl}_{3}\right)$ $\delta 142.9,142.1,139.0,137.1,136.2,130.3,129.6,129.3,128.5,120.0,112.9,102.1,21.1,19.7$. HRMS (ESI) m/z: [M + H] $]^{+}$Calcd for $\mathrm{C}_{14} \mathrm{H}_{15} \mathrm{IN}$ 324.0249; Found 324.0257. 


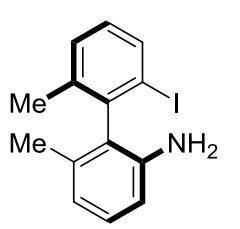

$11 \mathrm{a}$

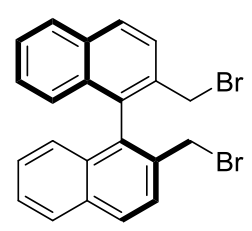

${ }^{B r}$

$\mathrm{K}_{2} \mathrm{CO}_{3}(30 \%$ aq. $), \mathrm{Nal}$,
actone, $40^{\circ} \mathrm{C}$

To a $25 \mathrm{~mL}$ Schlenk tube was added $11 \mathrm{a}$ (35.5 mg, $0.11 \mathrm{mmol}, 1.10$ equiv), 30\% potassium carbonate solution $(1.0 \mathrm{~mL}),(S)-2,2$ '-bis(bromomethyl)-1,1'-binaphthalene (44.0 $\mathrm{mg}, 0.10$ mmol, 1.00 equiv) and acetone $(1.0 \mathrm{~mL})$ under nitrogen atmosphere. After stirring at room temperature for $1 \mathrm{~h}$, sodium iodide $(37.5 \mathrm{mg}, 0.25 \mathrm{mmol}, 2.50$ equiv) was added then the mixture was heated to $40{ }^{\circ} \mathrm{C}$ in oil bath and stirred for $84 \mathrm{~h}$. After complete consumption of starting material, the mixture was cooled to room temperature then diluted with $\mathrm{H}_{2} \mathrm{O}$, extracted with $\mathrm{CH}_{2} \mathrm{Cl}_{2}(10.0 \mathrm{~mL}$ x2), washed with brine then dried over anhydrous sodium sulfate. The solid $\mathrm{Na}_{2} \mathrm{SO}_{4}$ was removed by filtration. The filtrate was concentrated and the residue was purified by preparative thin-layer chromatography (hexanes) to afford compound 12a (47.9 mg, $80 \%, \mathrm{dr}>25: 1$, white powder $) .[\alpha]_{D}^{20}=-77\left(\mathrm{c}=1.22, \mathrm{CHCl}_{3}\right) .{ }^{1} \mathbf{H} \mathbf{N M R}\left(400 \mathrm{MHz}, \mathrm{CDCl}_{3}\right) \delta$ $7.92(\mathrm{~d}, J=8.4,2 \mathrm{H}), 7.89(\mathrm{~d}, J=8.0 \mathrm{~Hz}, 2 \mathrm{H}), 7.71(\mathrm{~d}, J=7.6 \mathrm{~Hz}, 1 \mathrm{H}), 7.50-7.43(\mathrm{~m}, 6 \mathrm{H})$, 7.26-7.20 (m, 2H), 7.19-7.15 (m, 2H), $6.96(\mathrm{~d}, J=7.6 \mathrm{~Hz}, 1 \mathrm{H}), 6.81(\mathrm{t}, J=7.6 \mathrm{~Hz}, 1 \mathrm{H}), 6.75$ $(\mathrm{d}, J=8.4 \mathrm{~Hz}, 1 \mathrm{H}), 3.94(\mathrm{~d}, J=12.0 \mathrm{~Hz}, 2 \mathrm{H}), 3.49(\mathrm{~d}, J=12.0 \mathrm{~Hz}, 2 \mathrm{H}), 2.08(\mathrm{~s}, 3 \mathrm{H}), 2.00(\mathrm{~s}$, 3H). ${ }^{13} \mathbf{C}$ NMR (126 MHz, $\left.\mathrm{CDCl}_{3}\right) \delta$ 149.0, 144.0, 138.7, 137.39, 137.34, 136.7, 134.7, 133.8, 133.0, 131.2, 130.2, 128.7, 128.31, 128.24, 128.0, 127.6, 127.4, 125.7, 125.4, 124.1, 117.6, 104.8, 53.8, 21.2, 20.5. HRMS (ESI) $\mathrm{m} / \mathrm{z}$ : $[\mathrm{M}+\mathrm{H}]^{+}$Calcd for $\mathrm{C}_{36} \mathrm{H}_{29} \mathrm{IN}$ 602.1345; Found 602.1345.

Synthesis of Compound $\mathbf{1 3 \mathbf { a } ^ { 2 }}$

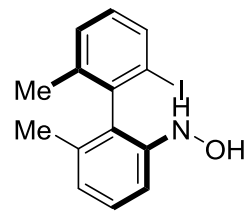

$10 \mathrm{a}$

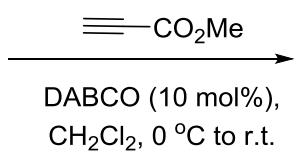

$\mathrm{CH}_{2} \mathrm{Cl}_{2}, 0^{\circ} \mathrm{C}$ to r.t.

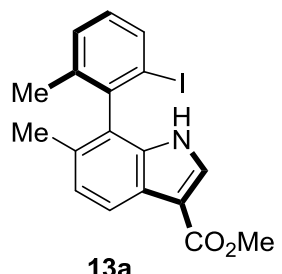

$13 a$ 
To a $25 \mathrm{~mL}$ Schlenk tube containing $10 \mathrm{a}$ (33.9 mg, $0.10 \mathrm{mmol}, 1.00$ equiv), DABCO (1.1 $\mathrm{mg}, 0.01 \mathrm{mmol}, 0.10$ equiv) and $\mathrm{CH}_{2} \mathrm{Cl}_{2}(2.0 \mathrm{~mL})$, methyl propiolate $(9.8 \mathrm{mg}, 0.11 \mathrm{mmol}, 1.10$ equiv) was added dropwise at $0{ }^{\circ} \mathrm{C}$ in ethanol bath under nitrogen atmosphere which was diluted with $\mathrm{CH}_{2} \mathrm{Cl}_{2}(1.0 \mathrm{~mL})$. The mixture was stirred at $0{ }^{\circ} \mathrm{C}$ for $10 \mathrm{~min}$ then warmed to room temperature and stirred for another $3 \mathrm{~h}$ until disappearance of starting material confirmed by TLC. The solvent was removed under vacuum, and the residue was purified by flash column chromatography on silica gel (hexane/ethyl acetate $=10: 1$ ) to afford the desired compound 13a (28.0 mg, 69\%, 97\% ee, pale yellow solid). $[\alpha]_{D}^{20}=+6.1\left(\mathrm{c}=0.57, \mathrm{CHCl}_{3}\right) . \mathrm{HPLC}$ conditions: Chiralpak AD-H, isopropanol $/$ hexane $=5: 95$, flow: $1.0 \mathrm{~mL} / \mathrm{min}, \lambda=254 \mathrm{~nm} .{ }^{1} \mathbf{H}$ NMR $(400$ $\left.\mathrm{MHz}, \mathrm{CDCl}_{3}\right) \delta 8.10(\mathrm{~d}, J=8.4 \mathrm{~Hz}, 1 \mathrm{H}), 8.01(\mathrm{~s}, 1 \mathrm{H}), 7.84(\mathrm{~d}, J=7.6 \mathrm{~Hz}, 1 \mathrm{H}), 7.79(\mathrm{~d}, J=2.8$ $\mathrm{Hz}, 1 \mathrm{H}), 7.32$ (d, $J=7.6 \mathrm{~Hz}, 1 \mathrm{H}), 7.23(\mathrm{~d}, J=8.4 \mathrm{~Hz}, 1 \mathrm{H}), 7.04$ (t, $J=7.6 \mathrm{~Hz}, 1 \mathrm{H}), 3.92$ (s, 3H), 2.12 (s, 3H), 1.97 (s, 3H). ${ }^{13} \mathbf{C}$ NMR (126 MHz, $\left.\mathrm{CDCl}_{3}\right) \delta 165.7,140.7,139.0,137.0$, 134.3, 130.6, $130.16130 .13,129.8,127.0,124.6,123.8,120.8,109.0,101.5,51.1,21.2,18.8$. HRMS (ESI) m/z: [M + Na $]^{+}$Calcd for $\mathrm{C}_{18} \mathrm{H}_{16} \mathrm{INO}_{2} \mathrm{Na}$ 428.0123; Found 428.0124

Synthesis of Compound $\mathbf{S 1}$

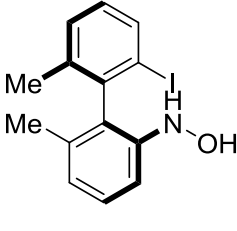

$10 \mathrm{a}$
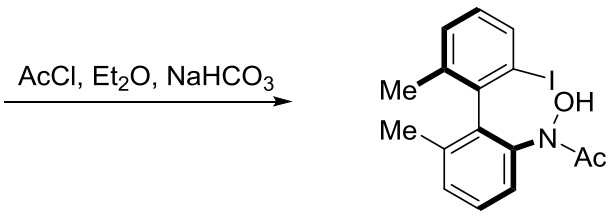

S1

To a $50 \mathrm{~mL}$ flask was added $\mathbf{1 0 a}(678.4 \mathrm{mg}, 2.00 \mathrm{mmol}, 1.00$ equiv), sodium bicarbonate (201.6 mg, $2.40 \mathrm{mmol}, 1.20$ equiv) and diethyl ether $(15.0 \mathrm{~mL})$ at $0{ }^{\circ} \mathrm{C}$ in ethanol bath under nitrogen atmosphere. The mixture was stirred for $3 \mathrm{~min}$, acetyl chloride $(188.4 \mathrm{mg}, 2.40 \mathrm{mmol}$, 1.20 equiv $)$ in diethyl ether $(5.0 \mathrm{~mL})$ was added dropwise, then the mixture was stirred at room temperature for $1 \mathrm{~h}$ until disappearance of starting material confirmed by TLC. The mixture was diluted with $\mathrm{H}_{2} \mathrm{O}$, extracted with ethyl acetate $(30.0 \mathrm{~mL}$ x2), washed with brine then dried over anhydrous sodium sulfate. The solid $\mathrm{Na}_{2} \mathrm{SO}_{4}$ was removed by filtration. The filtrate was 
concentrated and the residue was purified by flash column chromatography on silica gel (hexanes/ethyl acetate $=3: 1)$ to afford compound S1 $(681.7 \mathrm{mg}, 89 \%$, 99\% ee, orange solid). $[\alpha]_{D}^{20}=-0.2\left(\mathrm{c}=1.51, \mathrm{CHCl}_{3}\right)$. HPLC conditions: Chiralpak IC, isopropanol/hexane = 5:95, flow: $1.0 \mathrm{~mL} / \mathrm{min}, \lambda=254 \mathrm{~nm} .{ }^{1} \mathbf{H}$ NMR (400 MHz, $\left.\mathrm{CDCl}_{3}\right) \delta 8.29$ (bs, $\left.1 \mathrm{H}\right), 7.73$ (d, $J=$ $8.0 \mathrm{~Hz}, 1 \mathrm{H}), 7.40-7.37(\mathrm{~m}, 2 \mathrm{H}), 7.24(\mathrm{~d}, J=7.6 \mathrm{~Hz}, 1 \mathrm{H}), 7.17(\mathrm{~d}, J=6.4 \mathrm{~Hz}, 1 \mathrm{H}), 6.96(\mathrm{t}, J=$ $7.6 \mathrm{~Hz}, 1 \mathrm{H}), 2.19(\mathrm{~s}, 3 \mathrm{H}), 2.08(\mathrm{~s}, 3 \mathrm{H}), 2.01(\mathrm{~s}, 3 \mathrm{H}) .{ }^{13} \mathbf{C}$ NMR $\left(126 \mathrm{MHz}, \mathrm{CDCl}_{3}\right) \delta$ 165.6, 142.5, 141.0, 139.2, 138.5, 136.4, 135.9, 131.6, 129.8, 129.4, 128.5, 126.2, 99.7, 21.6, 21.1, 19.7. HRMS (ESI) m/z: $[\mathrm{M}+\mathrm{Na}]^{+}$Calcd for $\mathrm{C}_{16} \mathrm{H}_{16} \mathrm{INO}_{2} \mathrm{Na}$ 404.0123; Found 404.0127.

Synthesis of Compound $\mathbf{1 4 \mathbf { a } ^ { 3 }}$

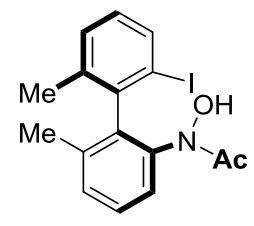

S1

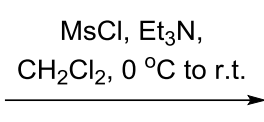

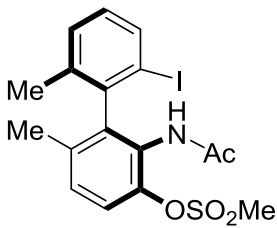

$14 a$

To a $25 \mathrm{~mL}$ Schlenk tube containing $\mathbf{S 1}(76.2 \mathrm{mg}, 0.20 \mathrm{mmol}, 1.00$ equiv), triethylamine (24.3 mg, 0.24 mmol, 1.2 equiv) and $\mathrm{CH}_{2} \mathrm{Cl}_{2}(5.0 \mathrm{~mL})$, was added methanesulfonyl chloride (24.1 mg, $0.21 \mathrm{mmol}, 1.05$ equiv) in $\mathrm{CH}_{2} \mathrm{Cl}_{2}(1.0 \mathrm{~mL})$ dropwise at $0{ }^{\circ} \mathrm{C}$ in ethanol bath under nitrogen atmosphere. The mixture was stirred for $10 \mathrm{~min}$ at $0{ }^{\circ} \mathrm{C}$ then warmed to room temperature stirred for another $30 \mathrm{~min}$ until disappearance of starting material confirmed by TLC. Then solvent was removed under vacuum, the residue was purified by flash column chromatography on silica gel (hexane/ethyl acetate $=10: 1)$ to afford $\mathbf{1 4 a}(86.1 \mathrm{mg}, 94 \%$, white powder). Regioselectivity (ortho:para) $=17: 1$, ortho: $97 \%$ ee, para: $99 \%$ ee. $[\alpha]_{D}^{20}=+78$ (c $\left.=1.00, \mathrm{CHCl}_{3}\right) . \mathrm{HPLC}$ conditions: Chiralpak AD-H, isopropanol/hexane $=10: 90$, flow: 1.0 $\mathrm{mL} / \mathrm{min}, \lambda=254 \mathrm{~nm} .{ }^{1} \mathbf{H}$ NMR $\left(400 \mathrm{MHz}, \mathrm{CDCl}_{3}\right) \delta 7.77(\mathrm{~d}, J=7.6 \mathrm{~Hz}, 1 \mathrm{H}), 7.41(\mathrm{~d}, J=8.4$ Hz, 1H), 7.31 (d, $J=8.4 \mathrm{~Hz}, 1 \mathrm{H}), 7.27(\mathrm{~d}, J=7.6 \mathrm{~Hz}, 1 \mathrm{H}), 7.00$ (d, $J=7.6 \mathrm{~Hz}, 1 \mathrm{H}), 6.48(\mathrm{~s}$, 1H), 3.20 (s, 3H), 2.05 (s, 3H), 1.97 (s, 3H), 1.88 (s, 2H). ${ }^{13} \mathbf{C ~ N M R ~ ( 1 2 6 ~ M H z , ~ C D C l ~} 3$ ) $\delta 168.7$, 
$143.8,143.5,140.9,138.9,136.5,135.9,130.3,130.0,129.7,127.6,121.8,99.8,38.0,23.0$, 21.1, 19.4. HRMS (ESI) m/z: $[\mathrm{M}+\mathrm{Na}]^{+}$Calcd for $\mathrm{C}_{17} \mathrm{H}_{18} \mathrm{INO}_{4} \mathrm{SNa} 481.9899$; Found 481.9903 .

Synthesis of Compound $\mathbf{1 4} \mathbf{b}^{4}$
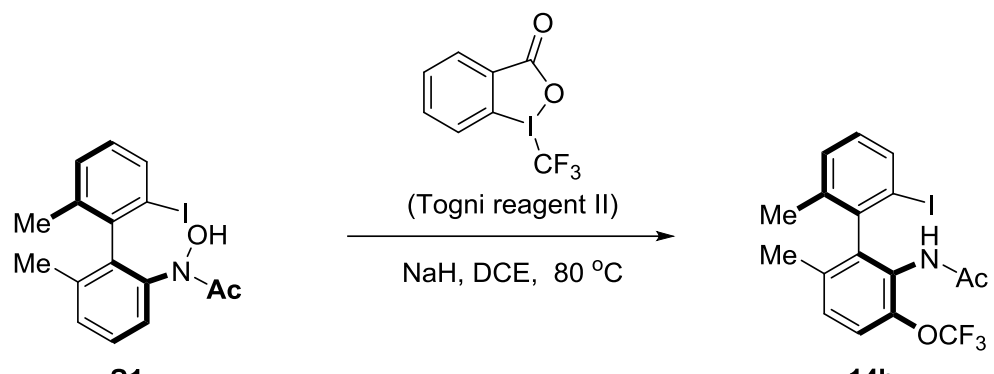

$14 b$

To a $25 \mathrm{~mL}$ Schlenk tube was added $\mathbf{S 1}$ (50.5 mg, $0.13 \mathrm{mmol}, 1.00$ equiv), Togni reagent II (50.2 mg, $0.16 \mathrm{mmol}, 1.2$ equiv) sodium hydride (60\% in oil, $6.4 \mathrm{mg}, 0.16 \mathrm{mmol}, 1.2$ equiv) and DCE $(2.0 \mathrm{~mL})$ under nitrogen atmosphere, the mixture was stirred at $80^{\circ} \mathrm{C}$ in oil bath for $22 \mathrm{~h}$. After complete consumption of starting material, the mixture was cooled to room temperature and then solvent was removed under vacuum, the residue was purified by flash column chromatography on silica gel (hexane/ethyl acetate $=3: 1$ ) to afford $\mathbf{1 4 b}(43.9 \mathrm{mg}, 74 \%$, 97\% ee, pale solid). $[\alpha]_{D}^{20}=+89\left(\mathrm{c}=1.23, \mathrm{CHCl}_{3}\right)$. HPLC conditions: Chiralpak AD-H, isopropanol/hexane $=5: 95$, flow: $1.0 \mathrm{~mL} / \mathrm{min}, \lambda=254 \mathrm{~nm} .{ }^{1} \mathbf{H} \mathbf{~ N M R}\left(500 \mathrm{MHz}, \mathrm{CDCl}_{3}\right) \delta 7.77$ $(\mathrm{d}, J=7.5 \mathrm{~Hz}, 1 \mathrm{H}), 7.34-7.26(\mathrm{~m}, 3 \mathrm{H}), 6.99(\mathrm{t}, J=7.5 \mathrm{~Hz}, 1 \mathrm{H}), 6.27(\mathrm{~s}, 1 \mathrm{H}), 2.03(\mathrm{~s}, 3 \mathrm{H}), 1.96$ $(\mathrm{s}, 3 \mathrm{H}), 1.88(\mathrm{~s}, 3 \mathrm{H}),{ }^{19} \mathbf{F}$ NMR $\left(471 \mathrm{MHz}, \mathrm{CDCl}_{3}\right) \delta-57.53 .{ }^{13} \mathbf{C}$ NMR $\left(126 \mathrm{MHz}, \mathrm{CDCl}_{3}\right) \delta$ $168.3,143.8,142.9,141.1,138.9,136.5,135.3,130.4,129.79,129.72,127.3,121.0,120.5$ (q, $\mathrm{J}=258.3 \mathrm{~Hz}$ ), 99.7, 22.8, 21.1, 19.3. HRMS (ESI) m/z: $[\mathrm{M}+\mathrm{Na}]^{+}$Calcd for $\mathrm{C}_{17} \mathrm{H}_{15} \mathrm{~F}_{3} \mathrm{INO}_{2} \mathrm{Na}$ 471.9997; Found 472.0000. 


\section{References}

1. Zhao, K.; Duan, L.; Xu, S.; Jiang, L.; Fu, Y.; Gu, Z. Chem 2018, 4, 599.

2. Feierfeil, J.; Magauer, T. Chem. Eur. J. 2018, 24, 1455.

3. Porzelle, A.; Woodrow, M. D.; Tomkinson, N. C. O. Eur. J. Org. Chem. 2008, 5135.

4. Hojczyk, K. N; Feng, P.; Zhan, C.; Ngai, M. Y. Angew. Chem. Int. Ed. 2014, 53, 14559.

\section{Method for crystal growth about 3a, 3o, 3u', 4t, 9a and 12a}

\section{For compound 3a, 3o, 3u', 4t, 12a:}

General procedure: The above compound (solid, $\sim 30 \mathrm{mg}$ ) was dissolved with minimal dichloromethane followed by about 1/5 (volume) hexane was added. The solvent was slowly volatizing under open air to afford crystalline, which was suitable for single crystal X-ray analysis.

\section{For compound 9a:}

General procedure: 9a (solid, $20 \mathrm{mg}$ ) was dissolved with minimal ethyl acetate followed by about 1/5 (volume) hexane was added. The solvent was slowly volatizing under open air to afford crystalline, which was suitable for single crystal X-ray analysis. 


\section{Crystal data and structure refinement for $3 a$}

Table S1 Crystal data and structure refinement for guj.

Identification code

guj

Empirical formula

$\mathrm{C}_{19} \mathrm{H}_{18} \mathrm{INO}_{5}$

Formula weight

467.24

Temperature/K

$100.00(10)$

Crystal system

monoclinic

Space group

$\mathrm{P} 2{ }_{1}$

$\mathrm{a} / \AA$

$10.1388(3)$

$\mathrm{b} / \AA$

$15.2537(4)$

c/A

$12.0272(4)$

$\alpha /{ }^{\circ}$

90

$\beta /{ }^{\circ}$

96.671(3)

$\gamma /{ }^{\circ}$

90

Volume $/ \AA^{3}$

1847.46(10)

$\mathrm{Z}$

4

$\rho_{\text {calc }} \mathrm{g} / \mathrm{cm}^{3}$

1.680

$\mu / \mathrm{mm}^{-1}$

1.763

$\mathrm{F}(000)$

928.0

Crystal size $/ \mathrm{mm}^{3}$

$0.11 \times 0.1 \times 0.08$

Radiation

$\operatorname{MoK} \alpha(\lambda=0.71073)$

$2 \Theta$ range for data collection $/{ }^{\circ} 4.044$ to 50

Index ranges

$-12 \leq \mathrm{h} \leq 12,-18 \leq \mathrm{k} \leq 18,-14 \leq 1 \leq 14$

Reflections collected

20197

Independent reflections

$6499\left[R_{\text {int }}=0.0408, R_{\text {sigma }}=0.0460\right]$

Data/restraints/parameters

$6499 / 1 / 459$

Goodness-of-fit on $\mathrm{F}^{2}$

1.058

Final $R$ indexes $[\mathrm{I}>=2 \sigma(\mathrm{I})] \quad \mathrm{R}_{1}=0.0280, \mathrm{wR}_{2}=0.0588$

Final $\mathrm{R}$ indexes [all data] $\quad \mathrm{R}_{1}=0.0297, \mathrm{wR}_{2}=0.0597$

Largest diff. peak/hole / e $\AA^{-3} 0.87 /-0.46$

Flack parameter

$-0.023(10)$ 


\section{Crystal data and structure refinement for 30}

Table S2 Crystal data and structure refinement for 3-152.

\begin{tabular}{|c|c|}
\hline Identification code & $3-152$ \\
\hline Empirical formula & $\mathrm{C}_{18} \mathrm{H}_{15} \mathrm{ClINO}_{5}$ \\
\hline Formula weight & 487.66 \\
\hline Temperature/K & $100.00(11)$ \\
\hline Crystal system & monoclinic \\
\hline Space group & $\mathrm{P} 2{ }_{1}$ \\
\hline $\mathrm{a} / \AA ̊$ & $8.6291(5)$ \\
\hline $\mathrm{b} / \AA ̊$ & $11.6603(6)$ \\
\hline$c / \AA$ & $18.9385(11)$ \\
\hline$\alpha /^{\circ}$ & 90 \\
\hline$\beta /{ }^{\circ}$ & $96.261(5)$ \\
\hline$\gamma /{ }^{\circ}$ & 90 \\
\hline Volume $/ \AA^{3}$ & 1894.19(18) \\
\hline $\mathrm{Z}$ & 4 \\
\hline$\rho_{\text {calc }} \mathrm{g} / \mathrm{cm}^{3}$ & 1.710 \\
\hline$\mu / \mathrm{mm}^{-1}$ & 1.860 \\
\hline $\mathrm{F}(000)$ & 960.0 \\
\hline Crystal size $/ \mathrm{mm}^{3}$ & $0.11 \times 0.1 \times 0.08$ \\
\hline Radiation & $\operatorname{Mo~K\alpha }(\lambda=0.71073)$ \\
\hline \multicolumn{2}{|c|}{$2 \Theta$ range for data collection $/{ }^{\circ} 4.108$ to 59.238} \\
\hline Index ranges & $-11 \leqslant \mathrm{~h} \leqslant 11,-15 \leqslant \mathrm{k} \leqslant 14,-25 \leqslant 1 \leqslant 22$ \\
\hline Reflections collected & 10987 \\
\hline Independent reflections & $7270\left[R_{\text {int }}=0.0476, R_{\text {sigma }}=0.0969\right]$ \\
\hline Data/restraints/parameters & $7270 / 50 / 475$ \\
\hline Goodness-of-fit on $\mathrm{F}^{2}$ & 1.038 \\
\hline Final $R$ indexes $[\mathrm{I}>=2 \sigma(\mathrm{I})]$ & $\mathrm{R}_{1}=0.0544, \mathrm{w}_{2}=0.1146$ \\
\hline Final $R$ indexes [all data] & $\mathrm{R}_{1}=0.0661, \mathrm{wR}_{2}=0.1253$ \\
\hline \multicolumn{2}{|c|}{ Largest diff. peak/hole / e $\AA^{-3} 1.82 /-1.13$} \\
\hline Flack parameter & $0.01(3)$ \\
\hline
\end{tabular}


Crystal data and structure refinement for $3 u^{\text {' }}$

Table S3 Crystal data and structure refinement for $3 Q$.

Identification code

3Q

Empirical formula

$\mathrm{C}_{16} \mathrm{H}_{16} \mathrm{INO}$

Formula weight

365.20

Temperature/K

$100.0(2)$

Crystal system

orthorhombic

Space group

$\mathrm{P} 2{ }_{1} 2_{1} 2_{1}$

$\mathrm{a} / \AA$

$8.8325(3)$

$\mathrm{b} / \AA$

$15.6906(5)$

c/Å

21.3761(8)

$\alpha /{ }^{\circ}$

90

$\beta /{ }^{\circ}$

90

$\gamma /{ }^{\circ}$

90

Volume $/ \AA^{3}$

2962.45(18)

Z

8

$\rho_{\text {calc }} \mathrm{g} / \mathrm{cm}^{3}$

1.638

$\mu / \mathrm{mm}^{-1}$

16.912

$\mathrm{F}(000)$

1440.0

Crystal size $/ \mathrm{mm}^{3}$

$0.13 \times 0.12 \times 0.1$

Radiation

$\mathrm{Cu} \mathrm{K \alpha}(\lambda=1.54184)$

$2 \Theta$ range for data collection $/{ }^{\circ} 6.988$ to 147.16

Index ranges

$-7 \leq \mathrm{h} \leq 10,-19 \leq \mathrm{k} \leq 13,-24 \leq 1 \leq 25$

Reflections collected

9291

Independent reflections

$5138\left[\mathrm{R}_{\text {int }}=0.0753, \mathrm{R}_{\text {sigma }}=0.0970\right]$

Data/restraints/parameters

$5138 / 19 / 337$

Goodness-of-fit on $\mathrm{F}^{2}$

1.045

Final R indexes $[\mathrm{I}>=2 \sigma(\mathrm{I})] \quad \mathrm{R}_{1}=0.0782, \mathrm{wR}_{2}=0.2028$

Final $\mathrm{R}$ indexes [all data] $\quad \mathrm{R}_{1}=0.0856, \mathrm{wR}_{2}=0.2166$

Largest diff. peak/hole / e $\AA^{-3} 0.92 /-1.11$

Flack parameter

$0.001(13)$ 


\section{Crystal data and structure refinement for $4 t$}

Table S4 Crystal data and structure refinement for 3-75.

\begin{tabular}{|c|c|}
\hline Identification code & $3-75$ \\
\hline Empirical formula & $\mathrm{C}_{31} \mathrm{H}_{26} \mathrm{INO}_{11} \mathrm{~S}_{2}$ \\
\hline Formula weight & 779.55 \\
\hline Temperature/K & $100.0(2)$ \\
\hline Crystal system & triclinic \\
\hline Space group & $\mathrm{P}-1$ \\
\hline $\mathrm{a} / \AA$ & $10.4982(8)$ \\
\hline $\mathrm{b} / \AA$ & $11.1323(9)$ \\
\hline$c / \AA$ & $15.0792(12)$ \\
\hline$\alpha /^{\circ}$ & $89.769(6)$ \\
\hline$\beta /{ }^{\circ}$ & $83.394(6)$ \\
\hline$\gamma /{ }^{\circ}$ & $69.155(7)$ \\
\hline Volume $/ \AA^{3}$ & $1634.7(2)$ \\
\hline $\mathrm{Z}$ & 2 \\
\hline$\rho_{\text {calc }} \mathrm{g} / \mathrm{cm}^{3}$ & 1.584 \\
\hline$\mu / \mathrm{mm}^{-1}$ & 1.168 \\
\hline $\mathrm{F}(000)$ & 784.0 \\
\hline Crystal size $/ \mathrm{mm}^{3}$ & $0.13 \times 0.12 \times 0.1$ \\
\hline Radiation & Mo $\mathrm{K} \alpha(\lambda=0.71073)$ \\
\hline \multicolumn{2}{|c|}{$2 \Theta$ range for data collection $/{ }^{\circ} 4.182$ to 50} \\
\hline Index ranges & $-10 \leqslant \mathrm{~h} \leqslant 12,-10 \leqslant \mathrm{k} \leqslant 13,-15 \leqslant 1 \leqslant 17$ \\
\hline Reflections collected & 10562 \\
\hline Independent reflections & $5721\left[\mathrm{R}_{\mathrm{int}}=0.0755, \mathrm{R}_{\text {sigma }}=0.0961\right]$ \\
\hline Data/restraints/parameters & $5721 / 7 / 419$ \\
\hline Goodness-of-fit on $\mathrm{F}^{2}$ & 1.022 \\
\hline Final $R$ indexes $[I>=2 \sigma(I)]$ & $\mathrm{R}_{1}=0.0500, \mathrm{wR}_{2}=0.1096$ \\
\hline Final $\mathrm{R}$ indexes [all data] & $\mathrm{R}_{1}=0.0586, \mathrm{wR}_{2}=0.1176$ \\
\hline Largest diff. peak/hole / e $\AA$ & $0.94 /-0.86$ \\
\hline
\end{tabular}




\section{Crystal data and structure refinement for 9a}

Table S5 Crystal data and structure refinement for 2-200.

\begin{tabular}{|c|c|}
\hline Identification code & $2-200$ \\
\hline Empirical formula & $\mathrm{C}_{30} \mathrm{H}_{28} \mathrm{INO}_{7}$ \\
\hline Formula weight & 641.43 \\
\hline Temperature/K & $100.01(10)$ \\
\hline Crystal system & triclinic \\
\hline Space group & $\mathrm{P}-1$ \\
\hline $\mathrm{a} / \AA ̊$ & $8.8149(5)$ \\
\hline $\mathrm{b} / \AA$ & $10.5473(6)$ \\
\hline $\mathrm{c} / \AA ̊$ & $15.6537(11)$ \\
\hline$\alpha /^{\circ}$ & $72.987(6)$ \\
\hline$\beta /{ }^{\circ}$ & $81.827(5)$ \\
\hline$\gamma /{ }^{\circ}$ & $80.495(5)$ \\
\hline Volume $/ \AA^{3}$ & $1365.78(15)$ \\
\hline $\mathrm{Z}$ & 2 \\
\hline$\rho_{\text {calc }} \mathrm{g} / \mathrm{cm}^{3}$ & 1.560 \\
\hline$\mu / \mathrm{mm}^{-1}$ & 1.221 \\
\hline $\mathrm{F}(000)$ & 648.0 \\
\hline Crystal size $/ \mathrm{mm}^{3}$ & $0.12 \times 0.11 \times 0.1$ \\
\hline Radiation & $\operatorname{Mo} \mathrm{K} \alpha(\lambda=0.71073)$ \\
\hline \multicolumn{2}{|c|}{$2 \Theta$ range for data collection $/{ }^{\circ} 4.234$ to 50} \\
\hline Index ranges & $-8 \leqslant \mathrm{~h} \leqslant 10,-12 \leqslant \mathrm{k} \leqslant 11,-17 \leqslant 1 \leqslant 18$ \\
\hline Reflections collected & 10106 \\
\hline Independent reflections & $4818\left[R_{\text {int }}=0.0334, R_{\text {sigma }}=0.0526\right]$ \\
\hline Data/restraints/parameters & $4818 / 0 / 357$ \\
\hline Goodness-of-fit on $\mathrm{F}^{2}$ & 1.054 \\
\hline Final R indexes $[\mathrm{I}>=2 \sigma(\mathrm{I})]$ & $\mathrm{R}_{1}=0.0304, \mathrm{wR}_{2}=0.0636$ \\
\hline Final R indexes [all data] & $\mathrm{R}_{1}=0.0350, \mathrm{wR}_{2}=0.0664$ \\
\hline Largest diff. peak/hole / e & $0.40 /-0.53$ \\
\hline
\end{tabular}




\section{Crystal data and structure refinement for $\mathbf{1 2 a}$}

Table S6 Crystal data and structure refinement for 134-2.

\begin{tabular}{|c|c|}
\hline Identification code & $134-2$ \\
\hline Empirical formula & $\mathrm{C}_{36} \mathrm{H}_{28} \mathrm{IN}$ \\
\hline Formula weight & 601.49 \\
\hline Temperature/K & $150.00(17)$ \\
\hline Crystal system & orthorhombic \\
\hline Space group & $\mathrm{P} 2{ }_{1} 2_{1} 2_{1}$ \\
\hline $\mathrm{a} / \AA ̊$ & $8.3055(12)$ \\
\hline $\mathrm{b} / \AA$ & $11.4354(13)$ \\
\hline$c / \AA$ & $28.367(3)$ \\
\hline$\alpha /^{\circ}$ & 90 \\
\hline$\beta /{ }^{\circ}$ & 90 \\
\hline$\gamma /{ }^{\circ}$ & 90 \\
\hline Volume $/ \AA^{3}$ & $2694.2(6)$ \\
\hline $\mathrm{Z}$ & 4 \\
\hline$\rho_{\text {calc }} \mathrm{g} / \mathrm{cm}^{3}$ & 1.483 \\
\hline$\mu / \mathrm{mm}^{-1}$ & 1.215 \\
\hline $\mathrm{F}(000)$ & 1216.0 \\
\hline Crystal size $/ \mathrm{mm}^{3}$ & $0.13 \times 0.12 \times 0.11$ \\
\hline Radiation & $\operatorname{Mo} K \alpha(\lambda=0.71073)$ \\
\hline \multicolumn{2}{|c|}{$2 \Theta$ range for data collection $/{ }^{\circ} 4.576$ to 59.058} \\
\hline Index ranges & $-8 \leqslant h \leqslant 11,-11 \leqslant \mathrm{k} \leqslant 15,-36 \leqslant 1 \leqslant 24$ \\
\hline Reflections collected & 14488 \\
\hline Independent reflections & $6320\left[R_{\text {int }}=0.0805, R_{\text {sigma }}=0.1026\right]$ \\
\hline Data/restraints/parameters & $6320 / 32 / 333$ \\
\hline Goodness-of-fit on $\mathrm{F}^{2}$ & 1.036 \\
\hline Final R indexes $[\mathrm{I}>=2 \sigma(\mathrm{I})]$ & $\mathrm{R}_{1}=0.0898, \mathrm{wR}_{2}=0.2175$ \\
\hline Final $\mathrm{R}$ indexes [all data] & $\mathrm{R}_{1}=0.1146, \mathrm{wR}_{2}=0.2476$ \\
\hline \multicolumn{2}{|c|}{ Largest diff. peak/hole / e $\AA^{-3} 1.98 /-1.48$} \\
\hline Flack/Hooft parameter & $0.01(3) / 0.05(2)$ \\
\hline
\end{tabular}


Thermal ellipsoid plot for the crystal structure for 3a, probability levels (50\%)

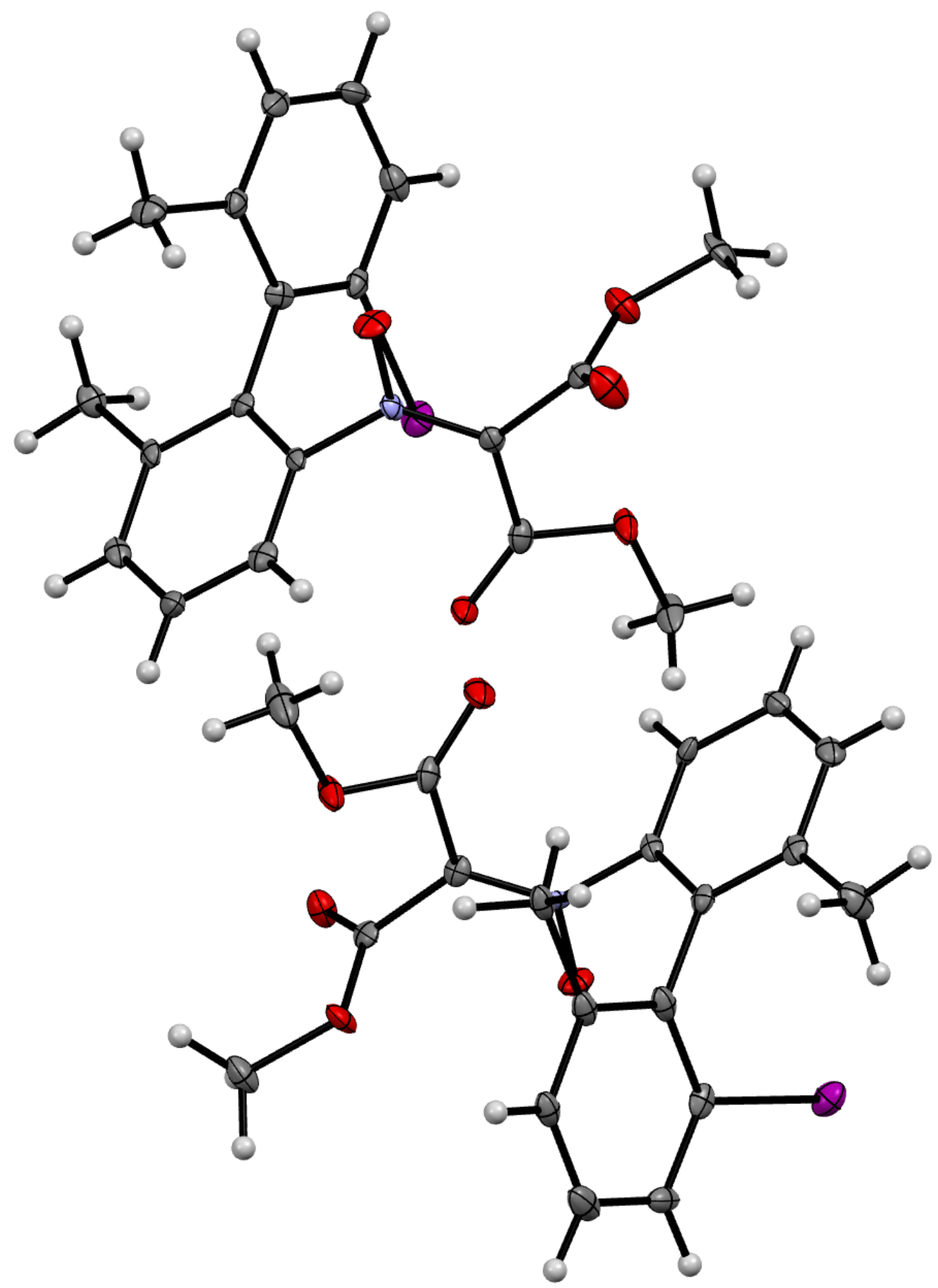


Thermal ellipsoid plot for the crystal structure for 3o, probability levels (50\%)

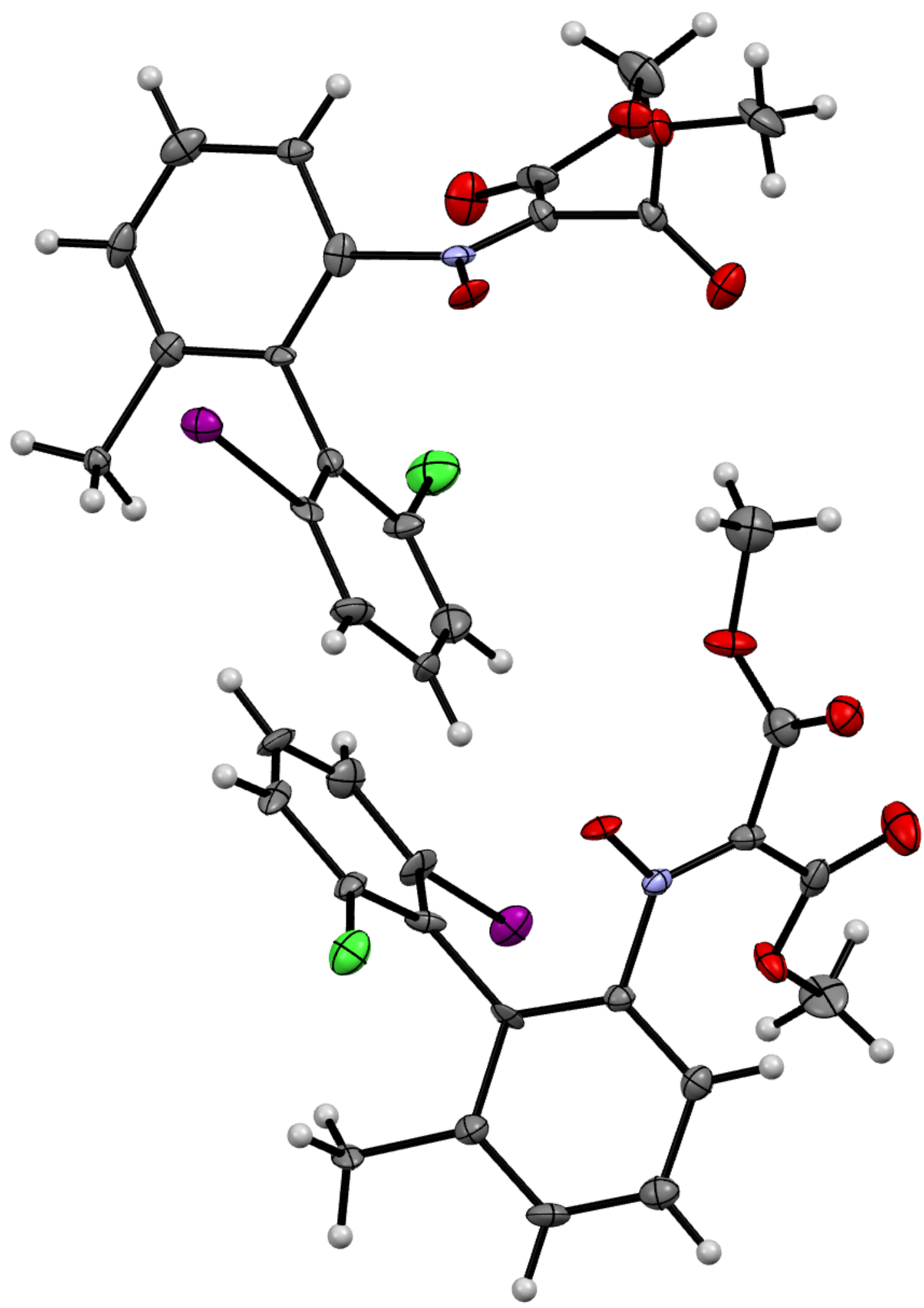


Thermal ellipsoid plot for the crystal structure for 3u', probability levels (50\%)

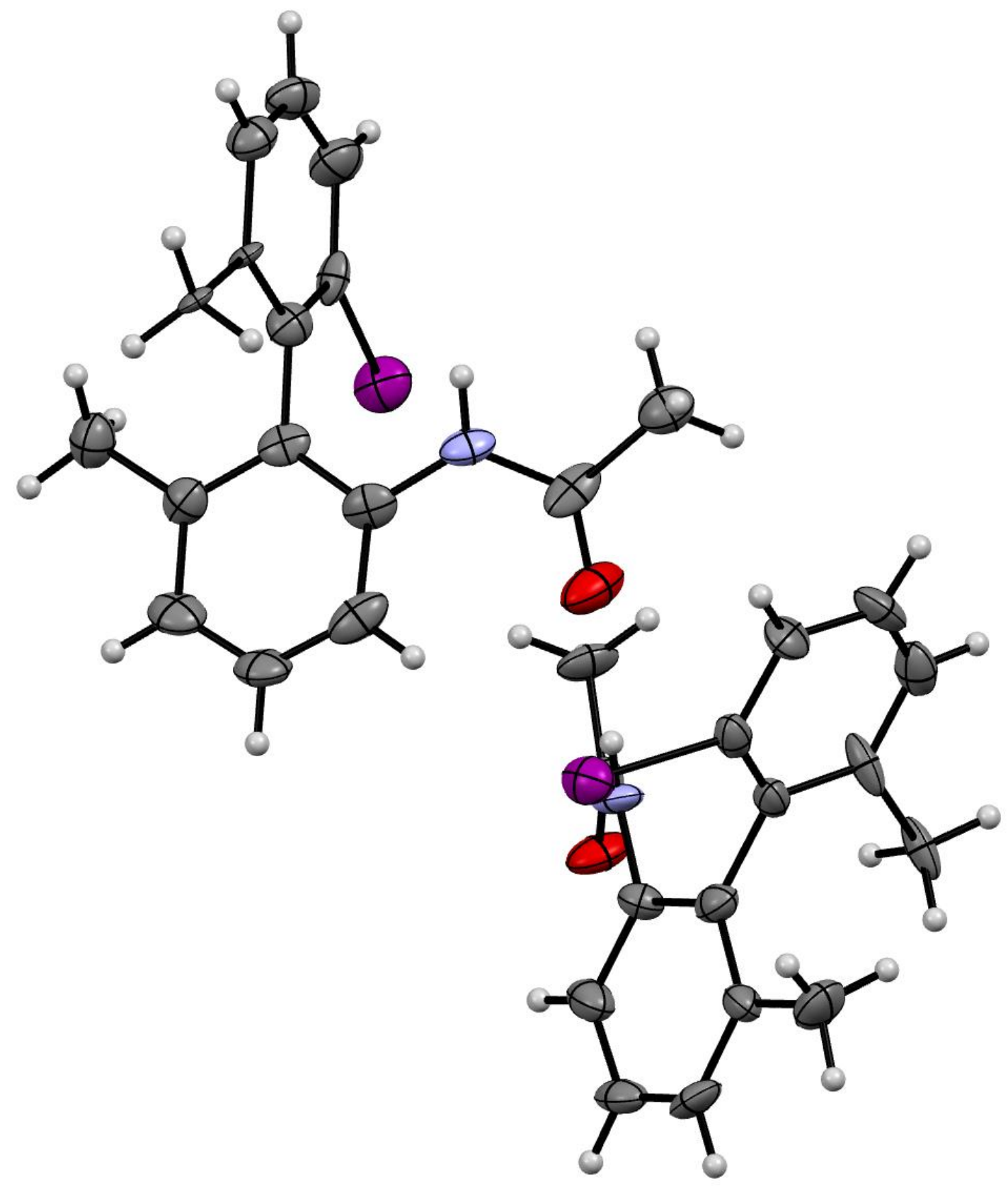


Thermal ellipsoid plot for the crystal structure for 4 t, probability levels (50\%)

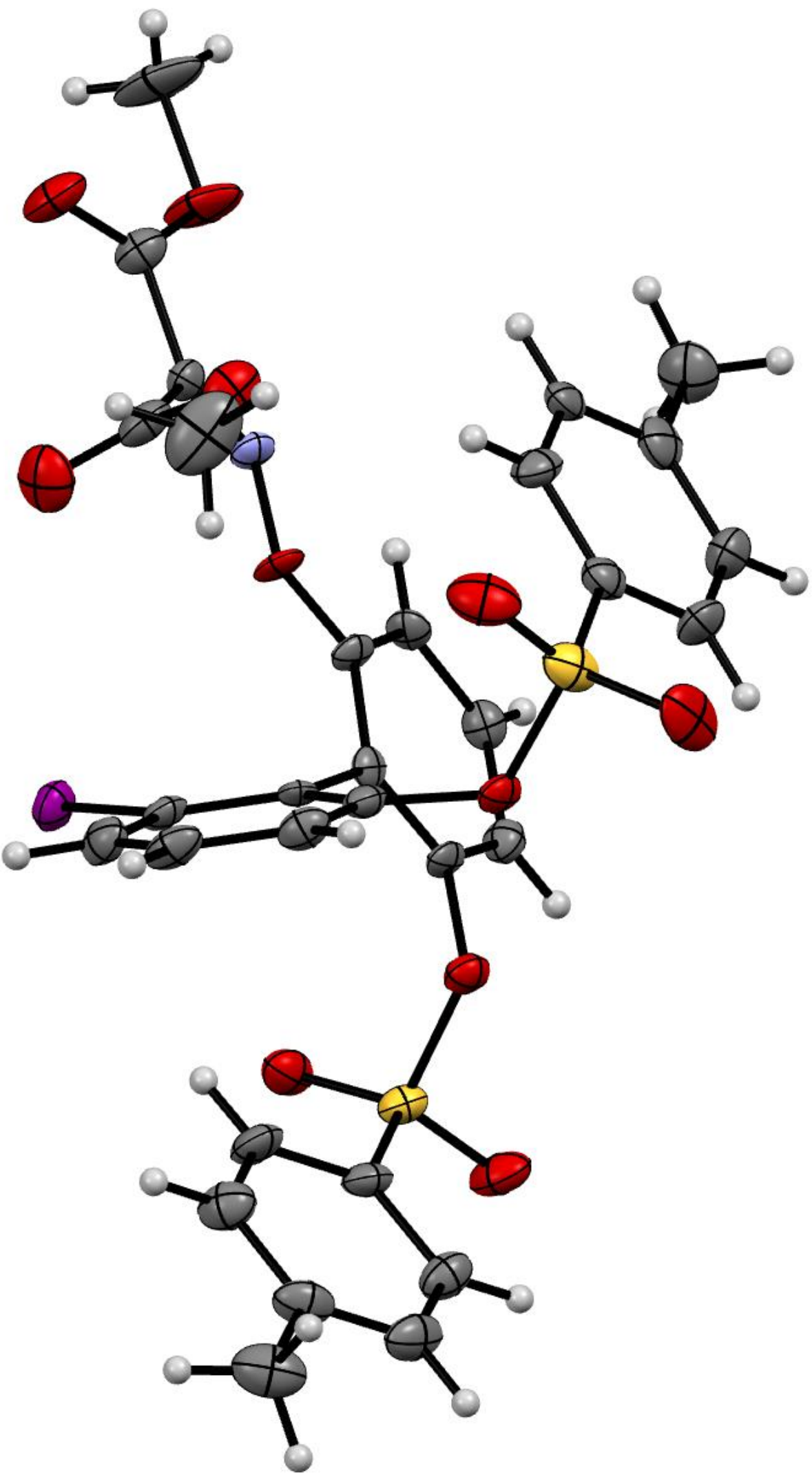


Thermal ellipsoid plot for the crystal structure for 9a, probability levels $(50 \%)$

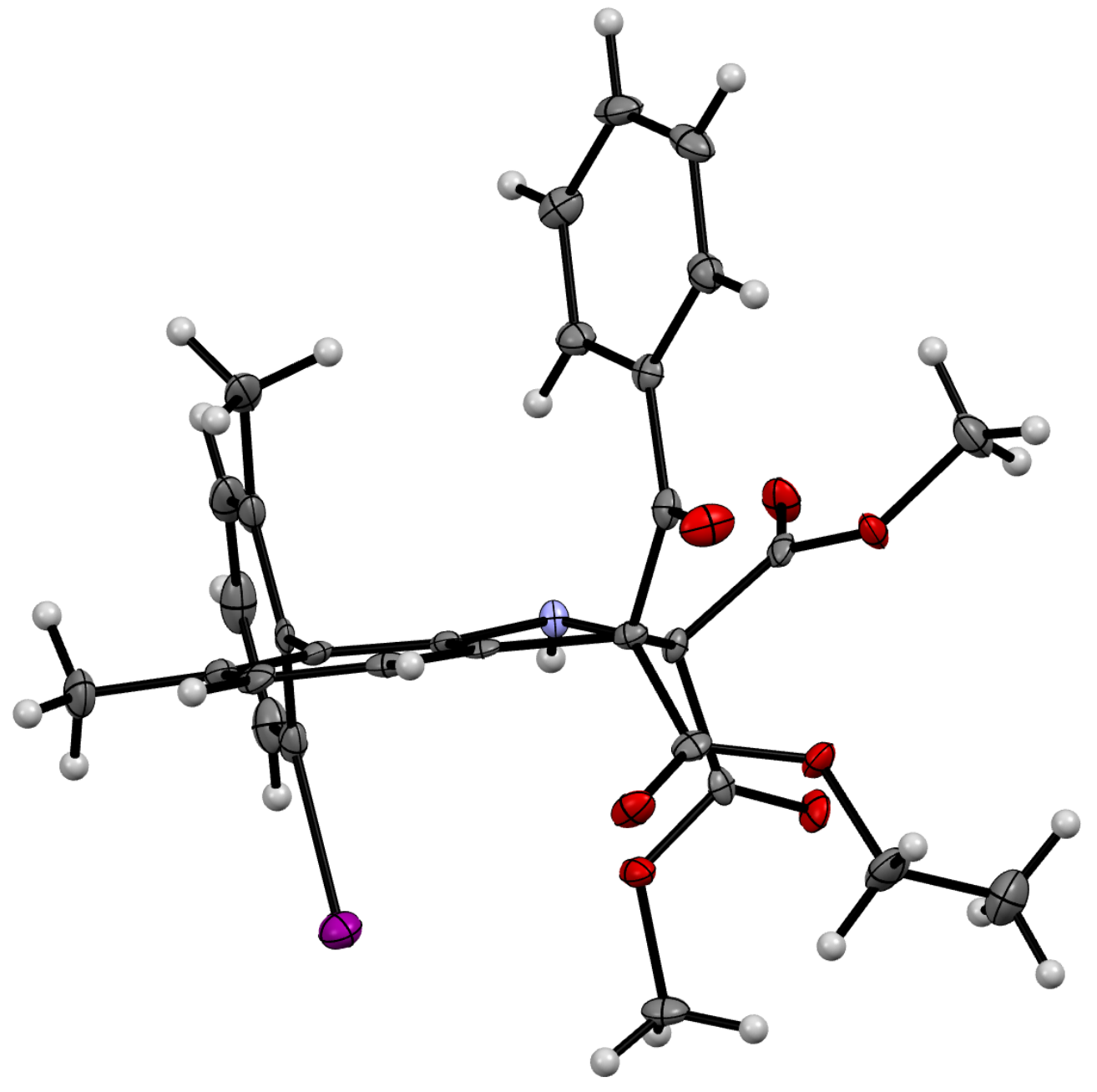


Thermal ellipsoid plot for the crystal structure for 12a, probability levels (50\%)

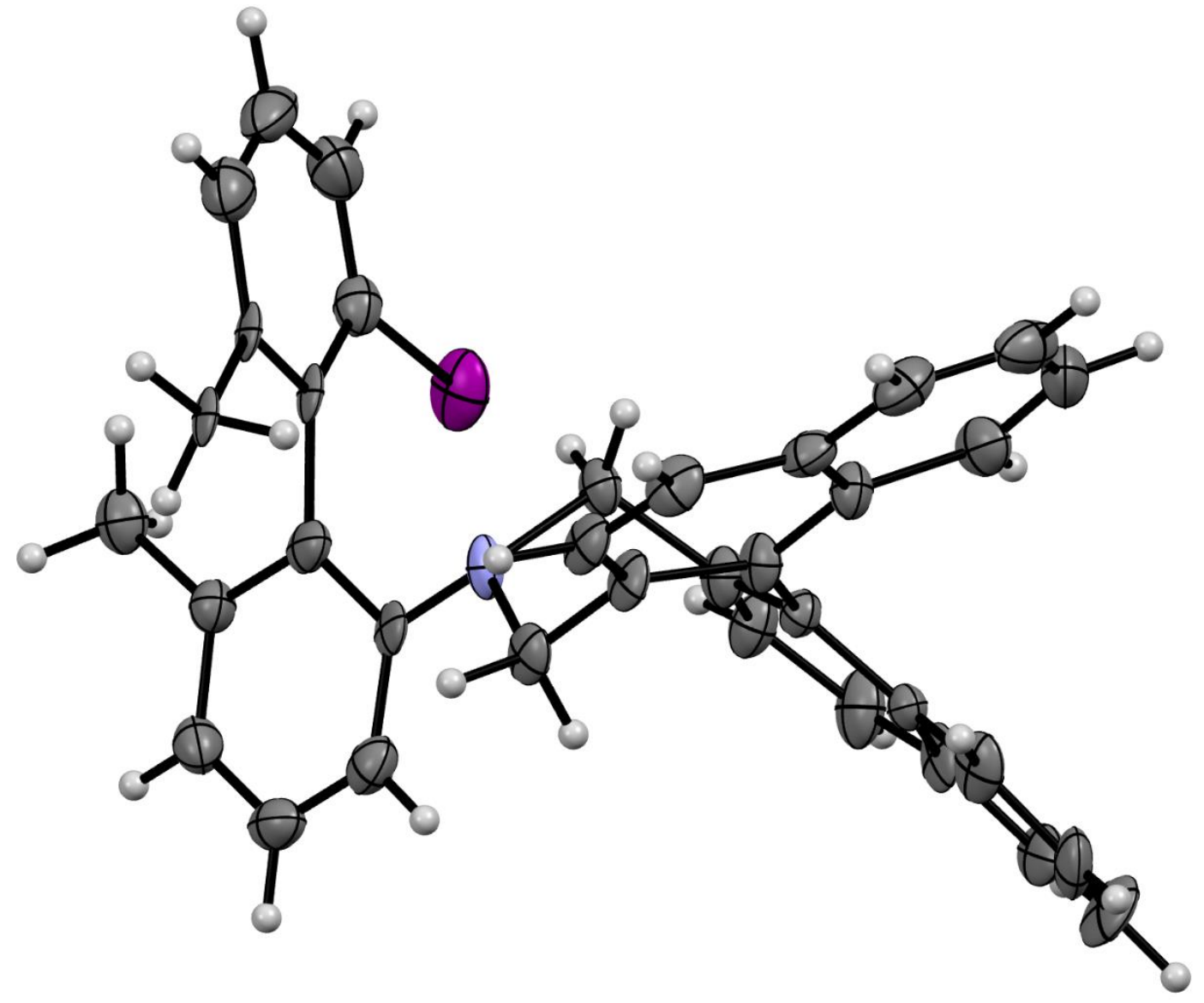




\section{Copies of NMR Spectroscopies}

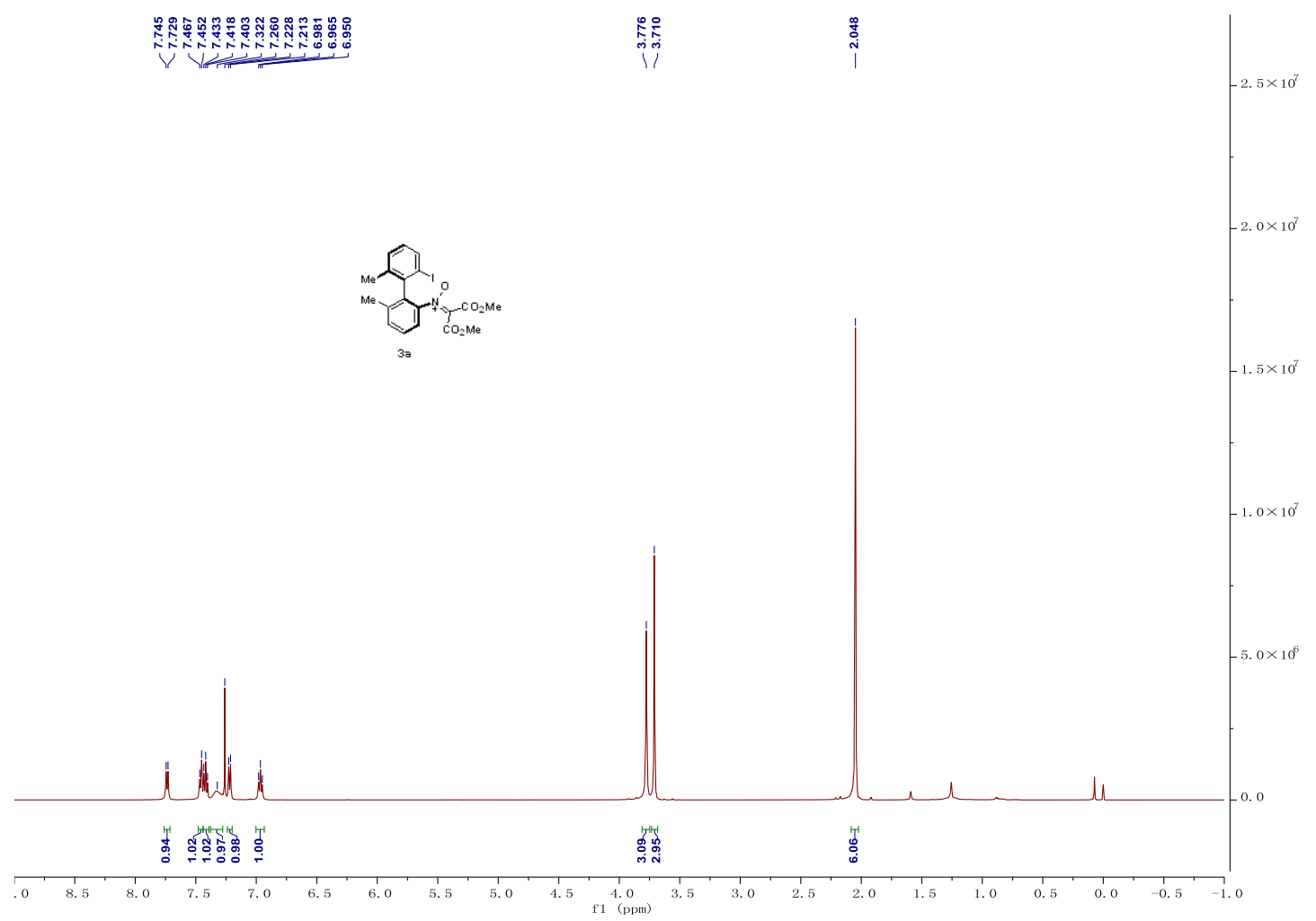

Figure S1. ${ }^{1} \mathrm{H}$ NMR spectra $\left(500 \mathrm{MHz}, \mathrm{CDCl}_{3}\right)$ of $\mathbf{3 a}$

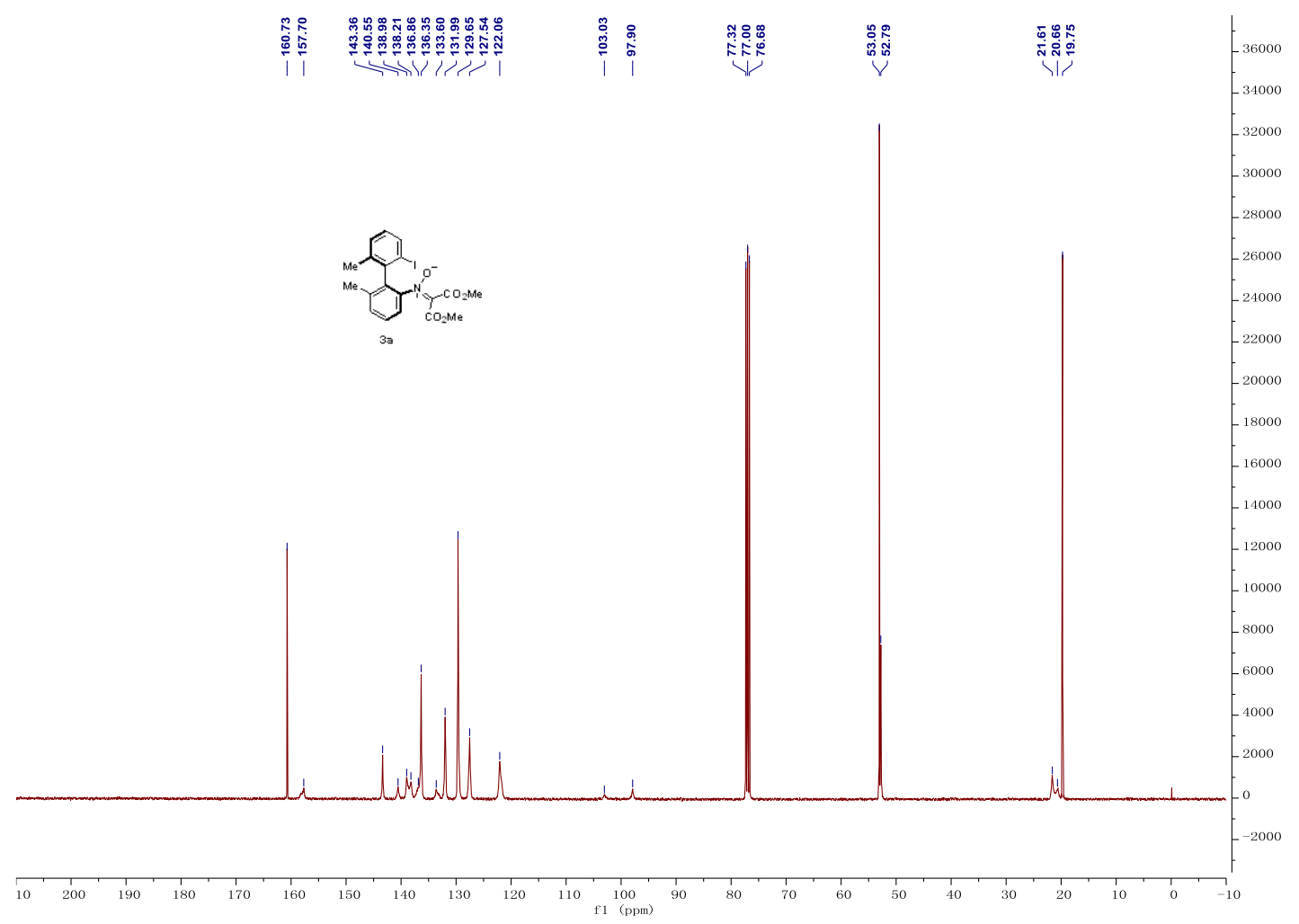

Figure S2. ${ }^{13} \mathrm{C}$ NMR spectra $\left(101 \mathrm{MHz}, \mathrm{CDCl}_{3}\right)$ of $\mathbf{3 a}$ 


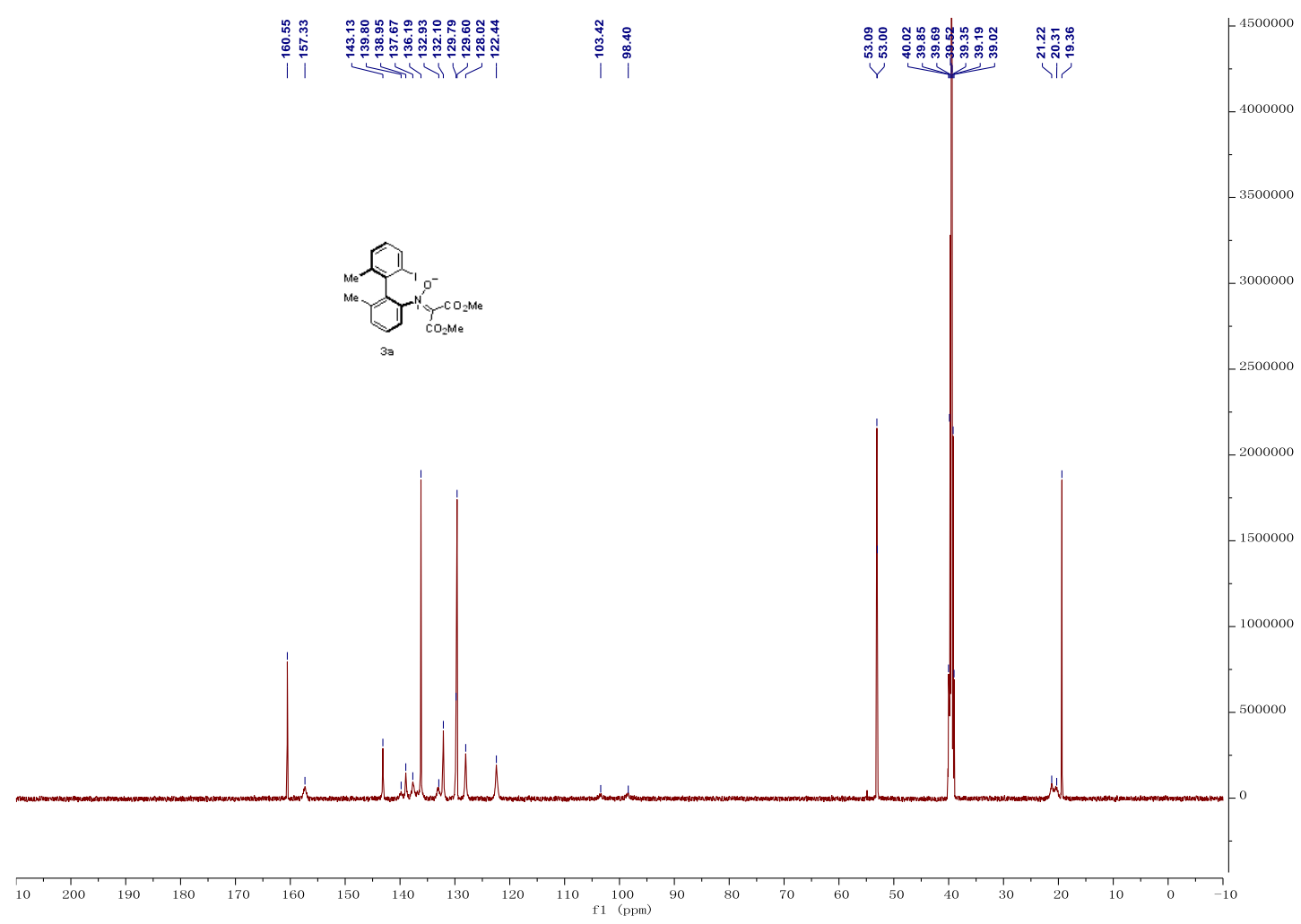

Figure S3. ${ }^{13} \mathrm{C}$ NMR spectra $\left(126 \mathrm{MHz}, \mathrm{DMSO}-d_{6}\right)$ of 3a

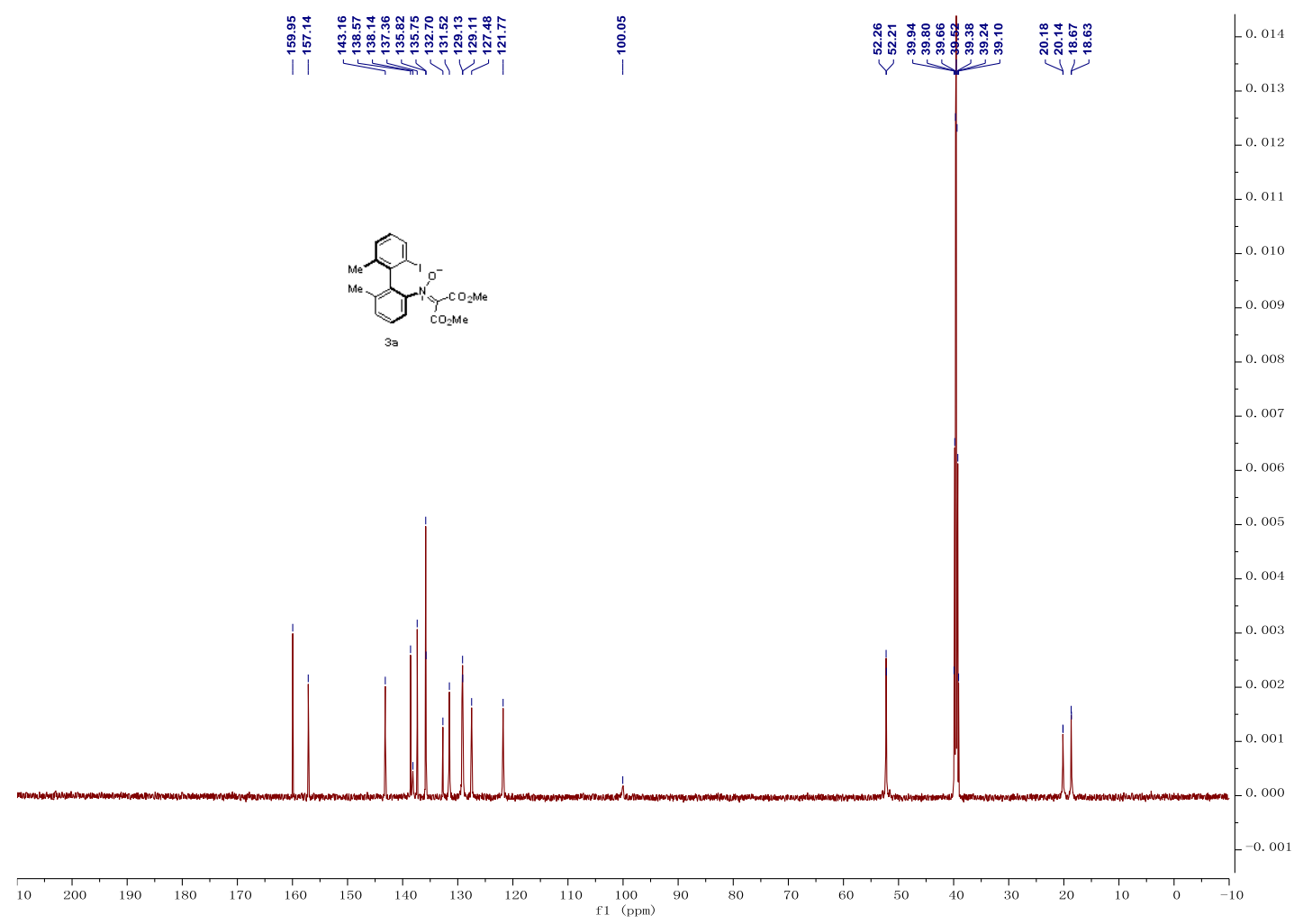

Figure S4. ${ }^{13} \mathrm{C}$ NMR spectra $\left(100{ }^{\circ} \mathbf{C}, 151 \mathrm{MHz}, \mathrm{DMSO}-d_{6}\right)$ of $\mathbf{3 a}$ 


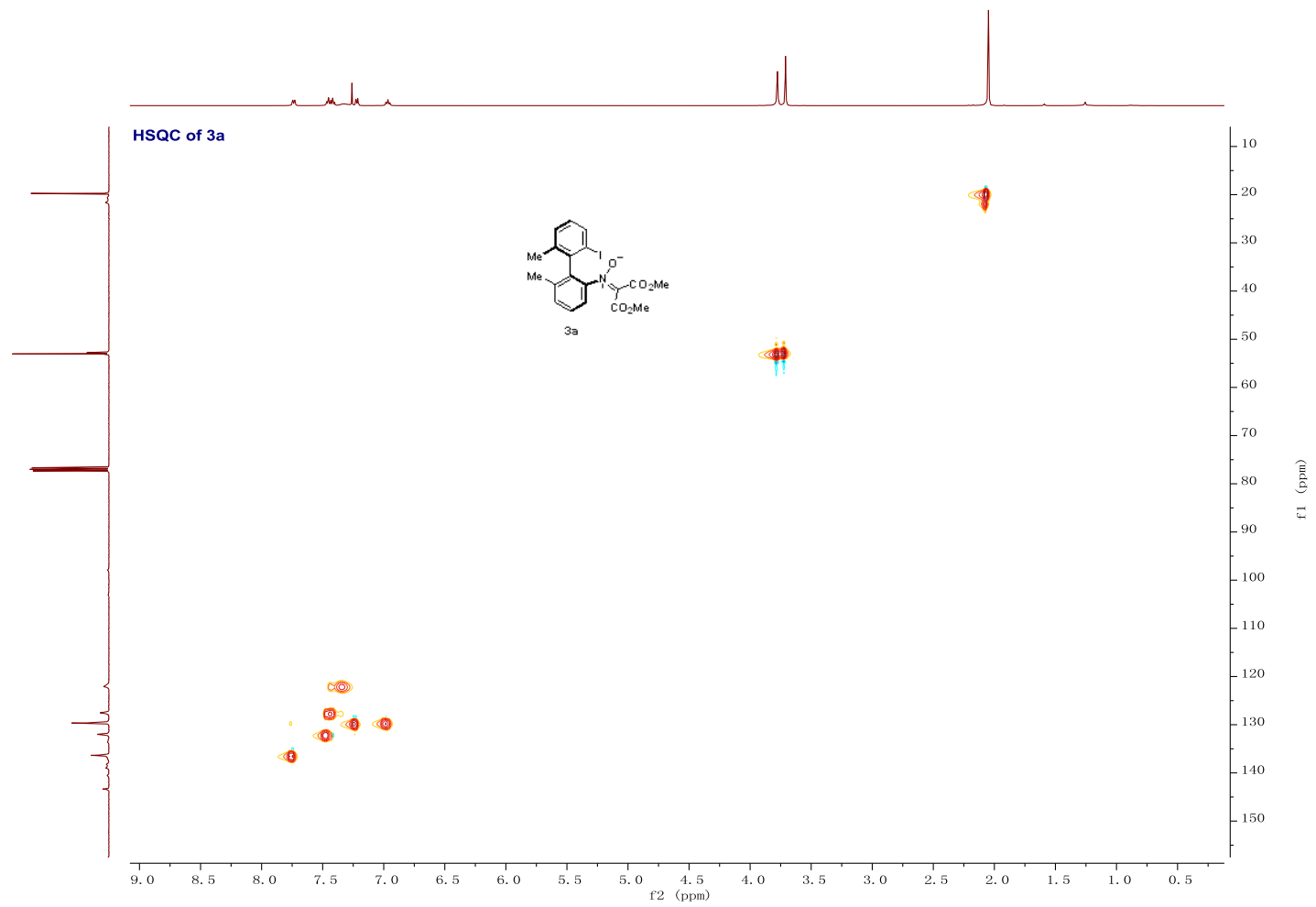

Figure S5. HSQC NMR spectra $\left(400 \mathrm{MHz}, 101 \mathrm{MHz}, \mathrm{CDCl}_{3}\right)$ of $\mathbf{3 a}$

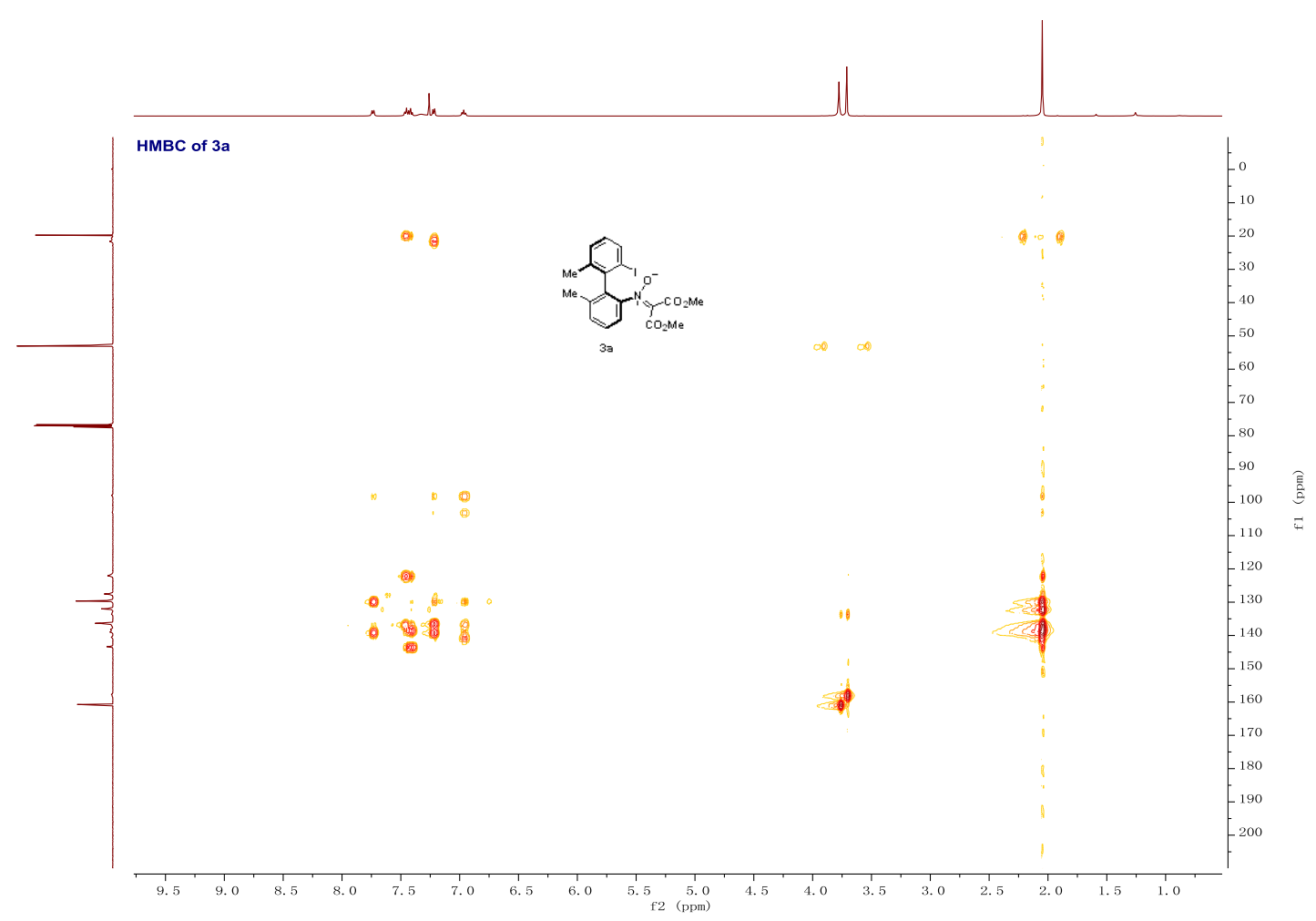

Figure S6. HMBC NMR spectra (400 MHz, $101 \mathrm{MHz}, \mathrm{CDCl}_{3}$ ) of 3a 


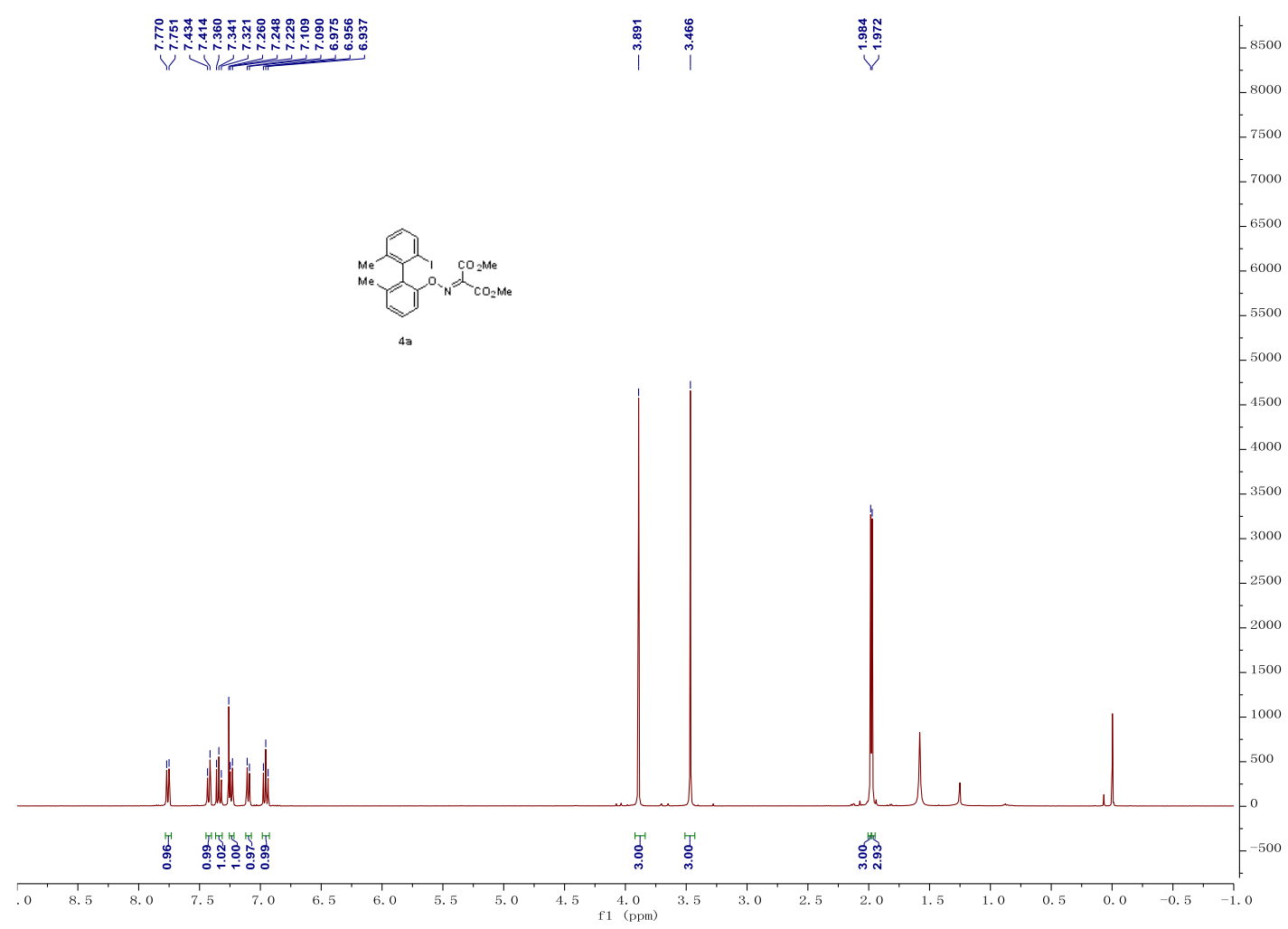

Figure S7. ${ }^{1} \mathrm{H}$ NMR spectra $\left(400 \mathrm{MHz}, \mathrm{CDCl}_{3}\right)$ of $\mathbf{4 a}$

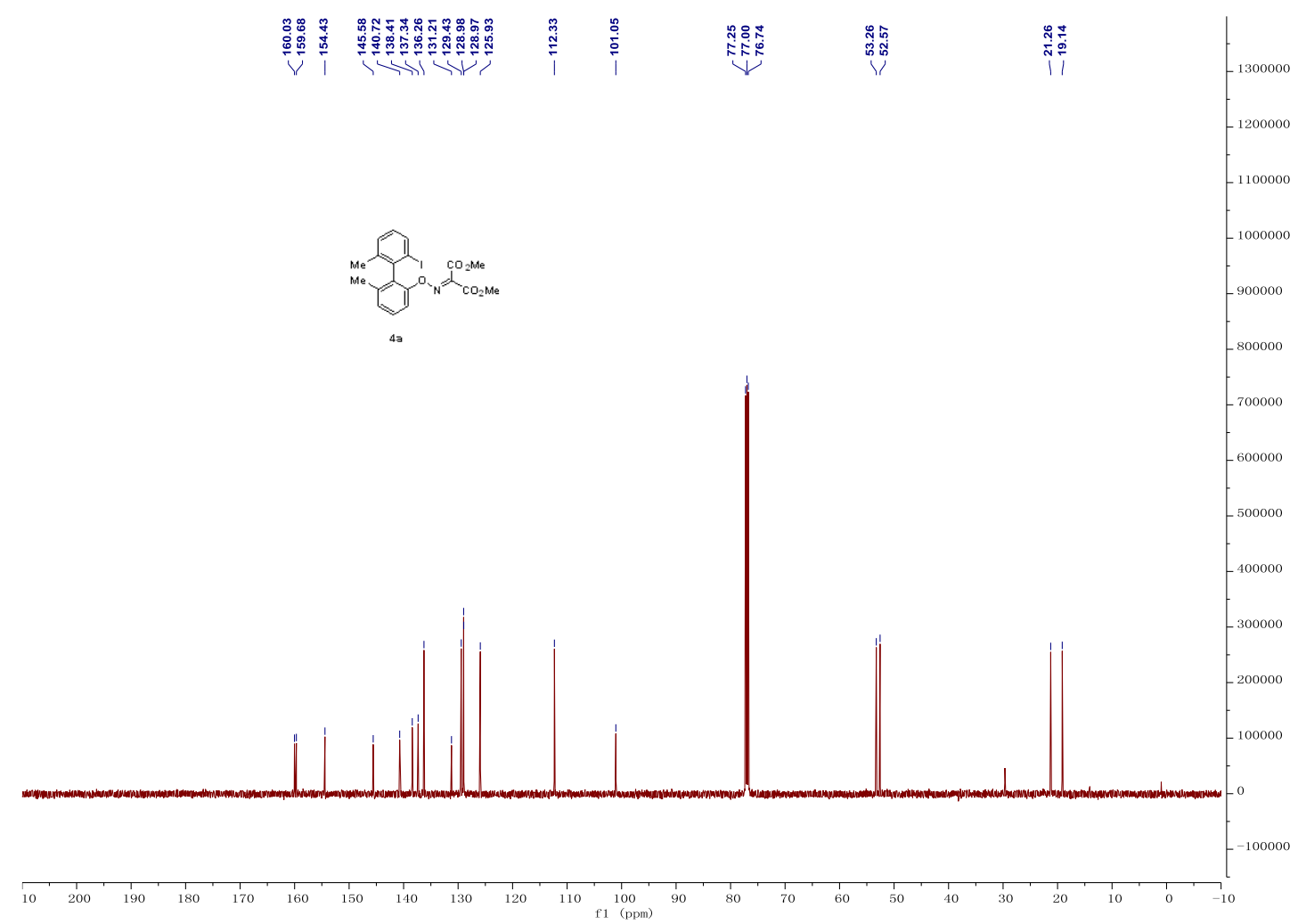

Figure S8. ${ }^{13} \mathrm{C}$ NMR spectra $\left(126 \mathrm{MHz}, \mathrm{CDCl}_{3}\right)$ of $\mathbf{4 a}$ 


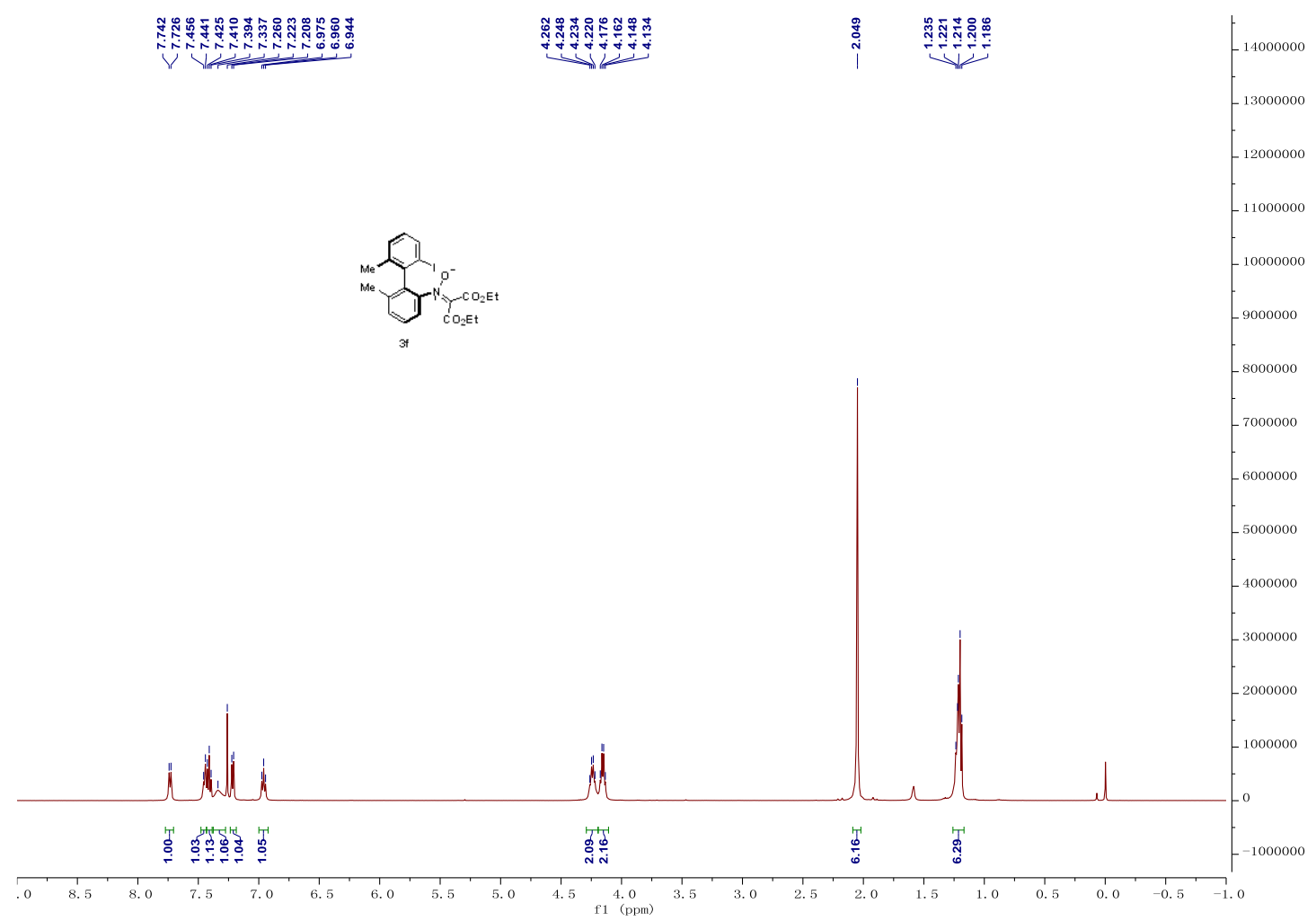

Figure S9. ${ }^{1} \mathrm{H}$ NMR spectra $\left(500 \mathrm{MHz}, \mathrm{CDCl}_{3}\right)$ of $\mathbf{3 f}$

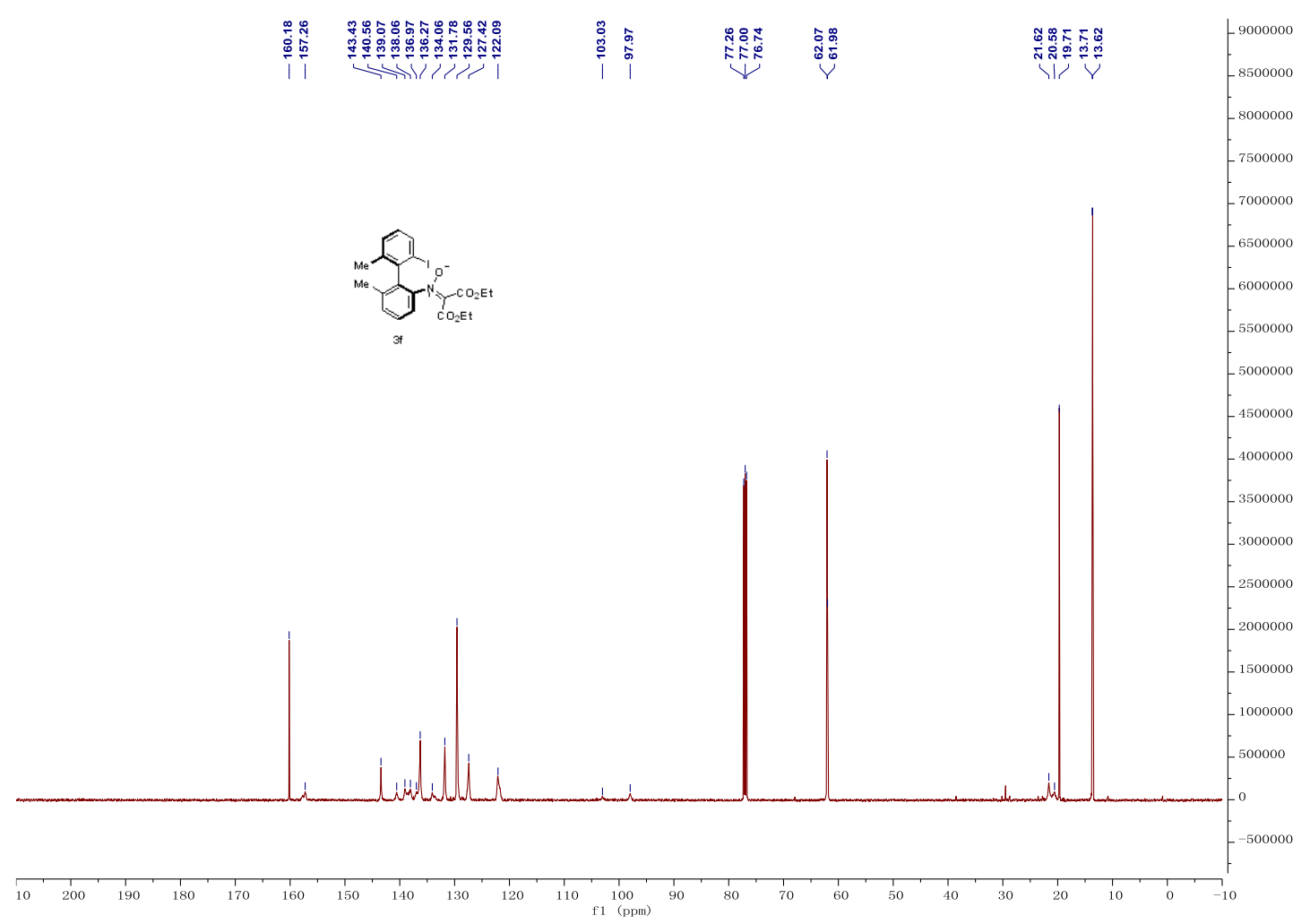

Figure S10. ${ }^{13} \mathrm{C}$ NMR spectra $\left(126 \mathrm{MHz}, \mathrm{CDCl}_{3}\right)$ of $\mathbf{3 f}$ 


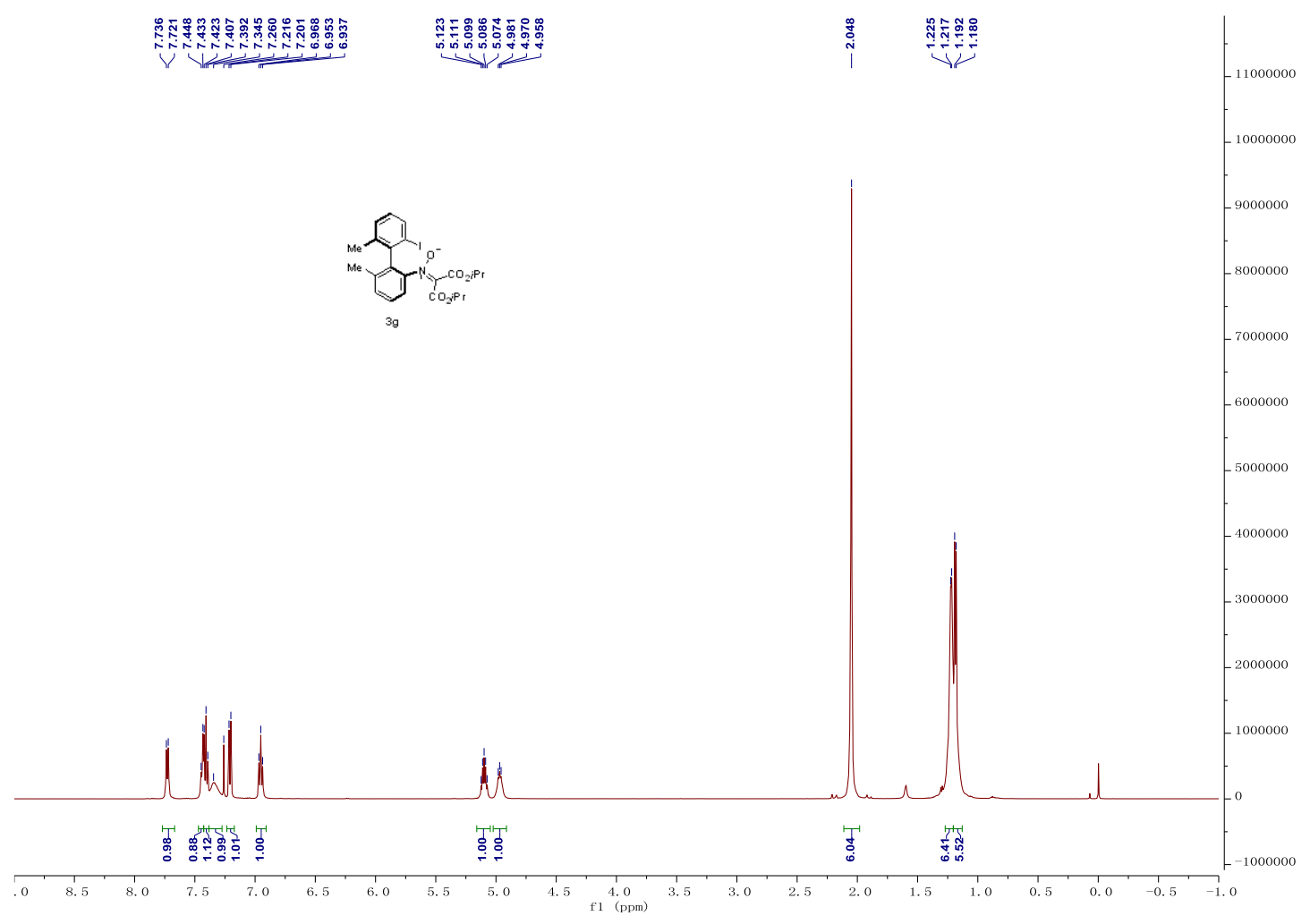

Figure S11. ${ }^{1} \mathrm{H}$ NMR spectra $\left(500 \mathrm{MHz}, \mathrm{CDCl}_{3}\right)$ of $\mathbf{3 g}$

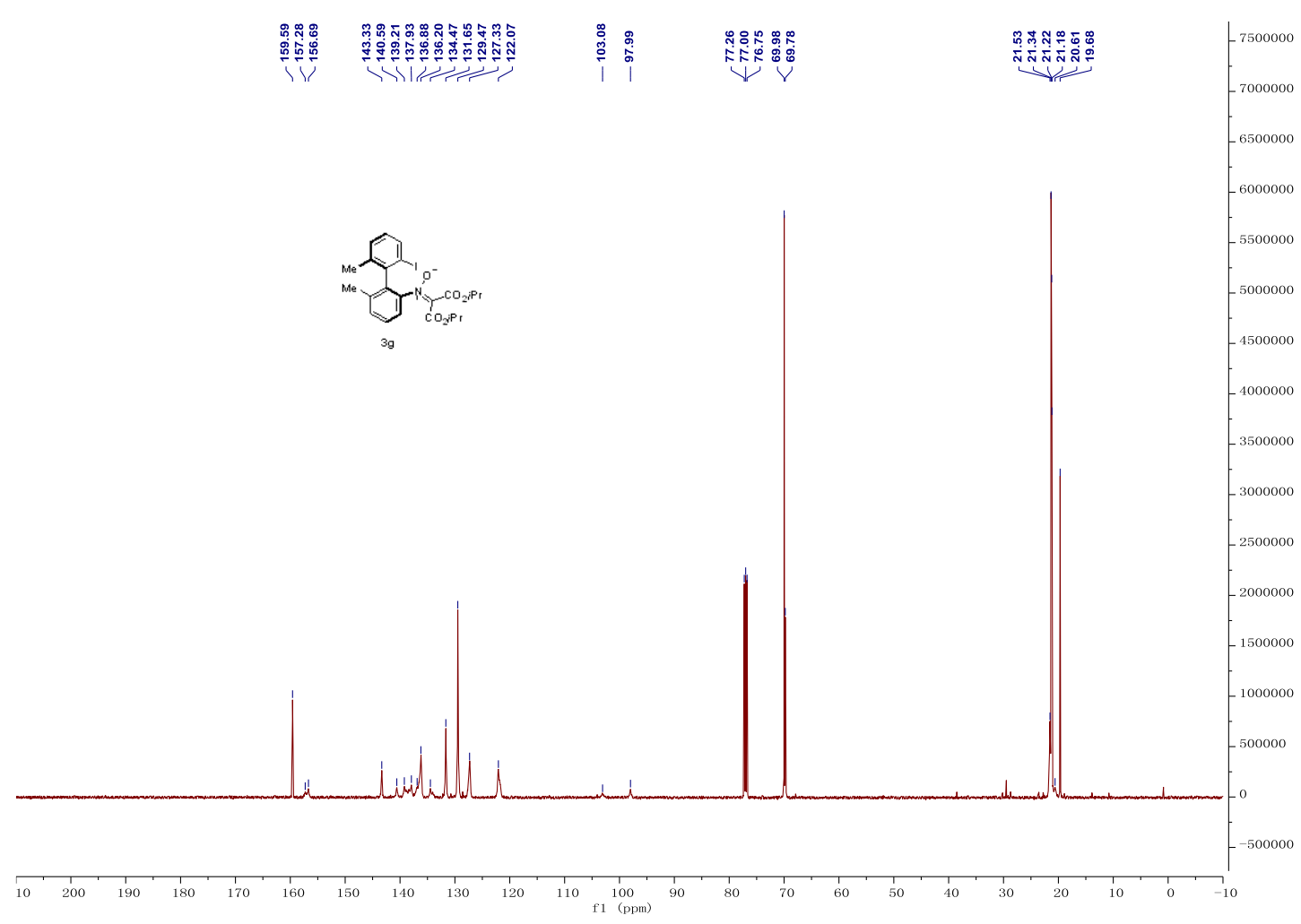

Figure S12. ${ }^{13} \mathrm{C}$ NMR spectra $\left(126 \mathrm{MHz}, \mathrm{CDCl}_{3}\right)$ of $\mathbf{3 g}$ 


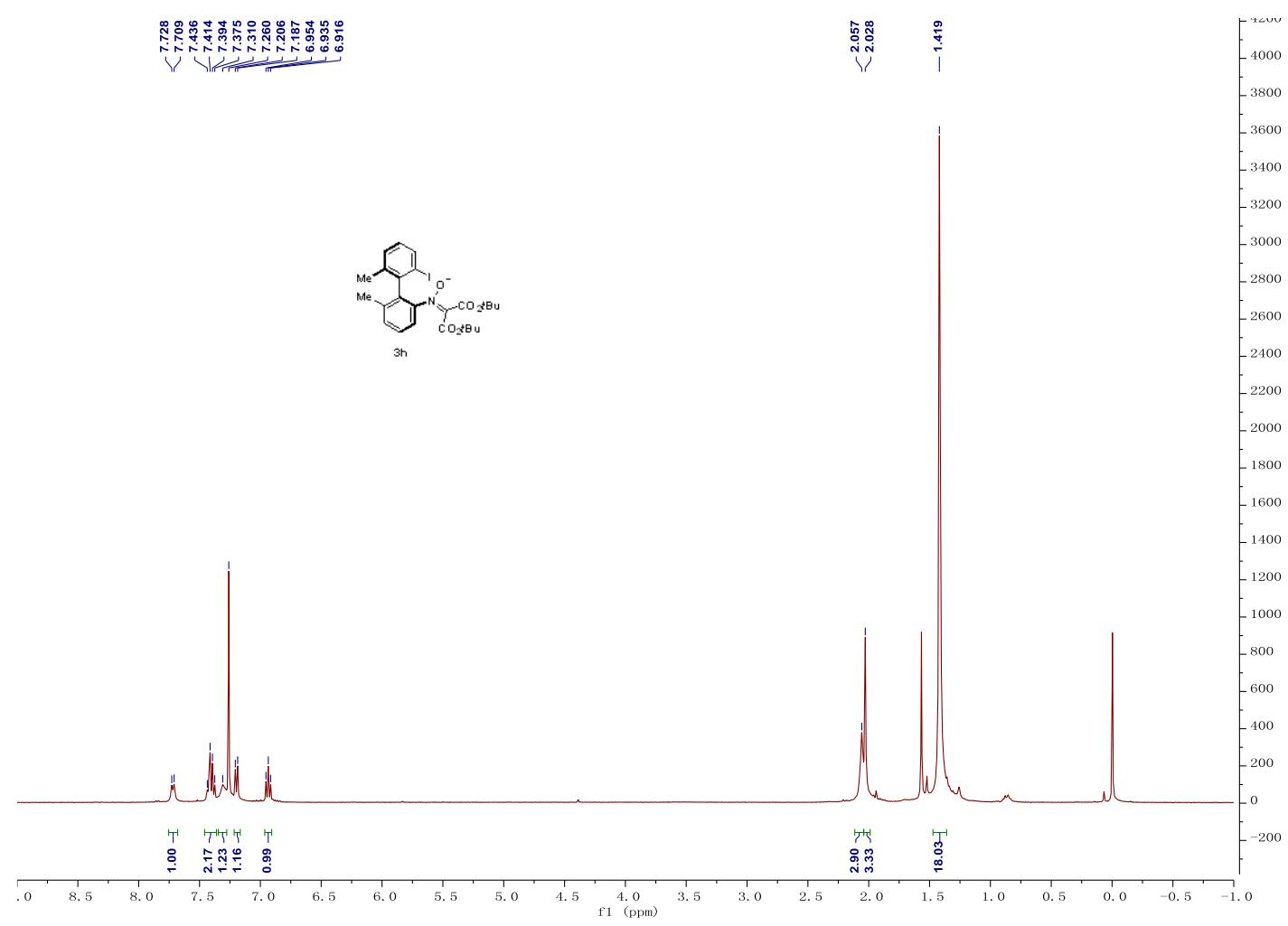

Figure S13. ${ }^{1} \mathrm{H}$ NMR spectra $\left(400 \mathrm{MHz}, \mathrm{CDCl}_{3}\right)$ of $\mathbf{3 h}$

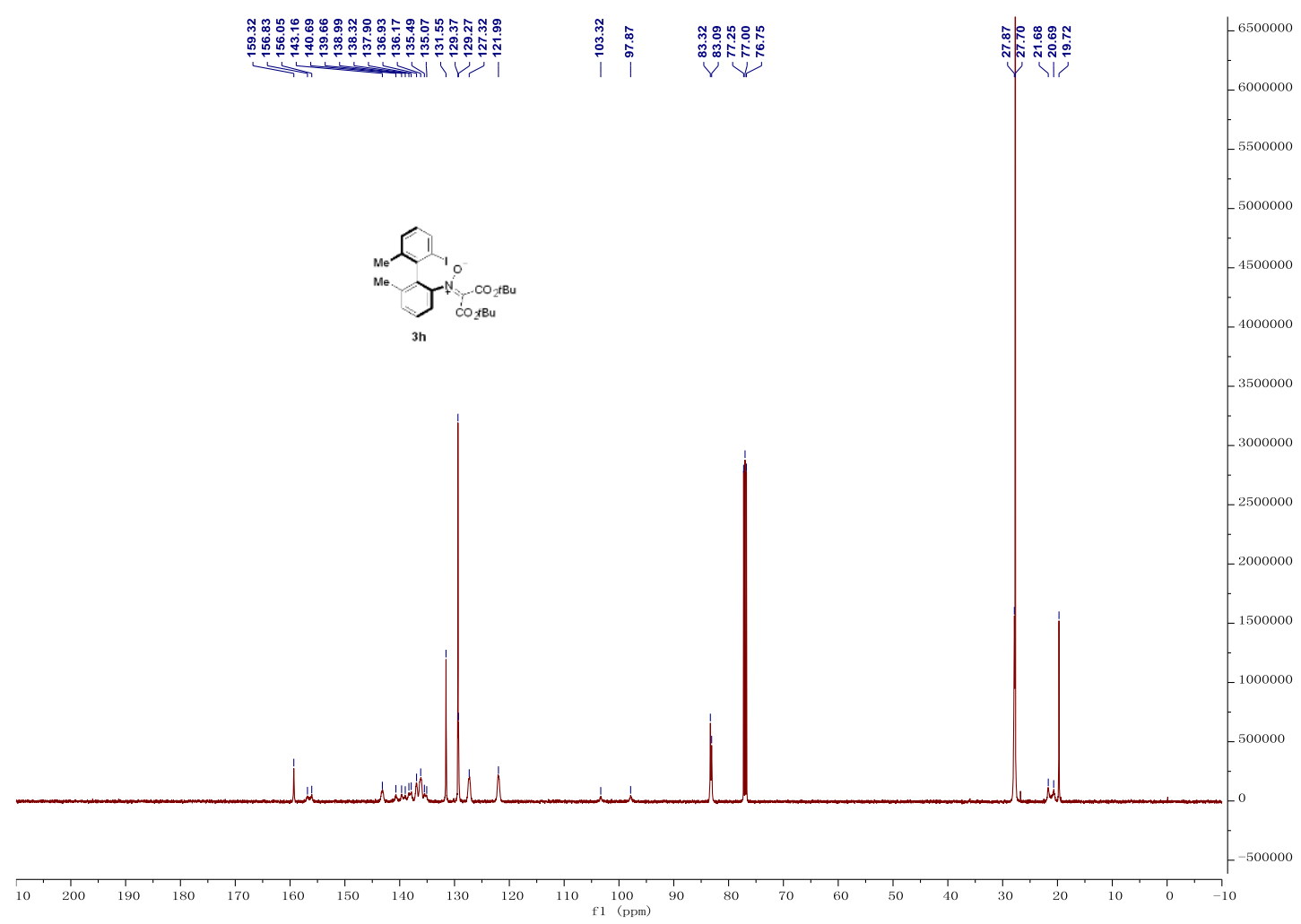

Figure S14. ${ }^{13} \mathrm{C}$ NMR spectra $\left(126 \mathrm{MHz}, \mathrm{CDCl}_{3}\right)$ of $\mathbf{3 h}$ 


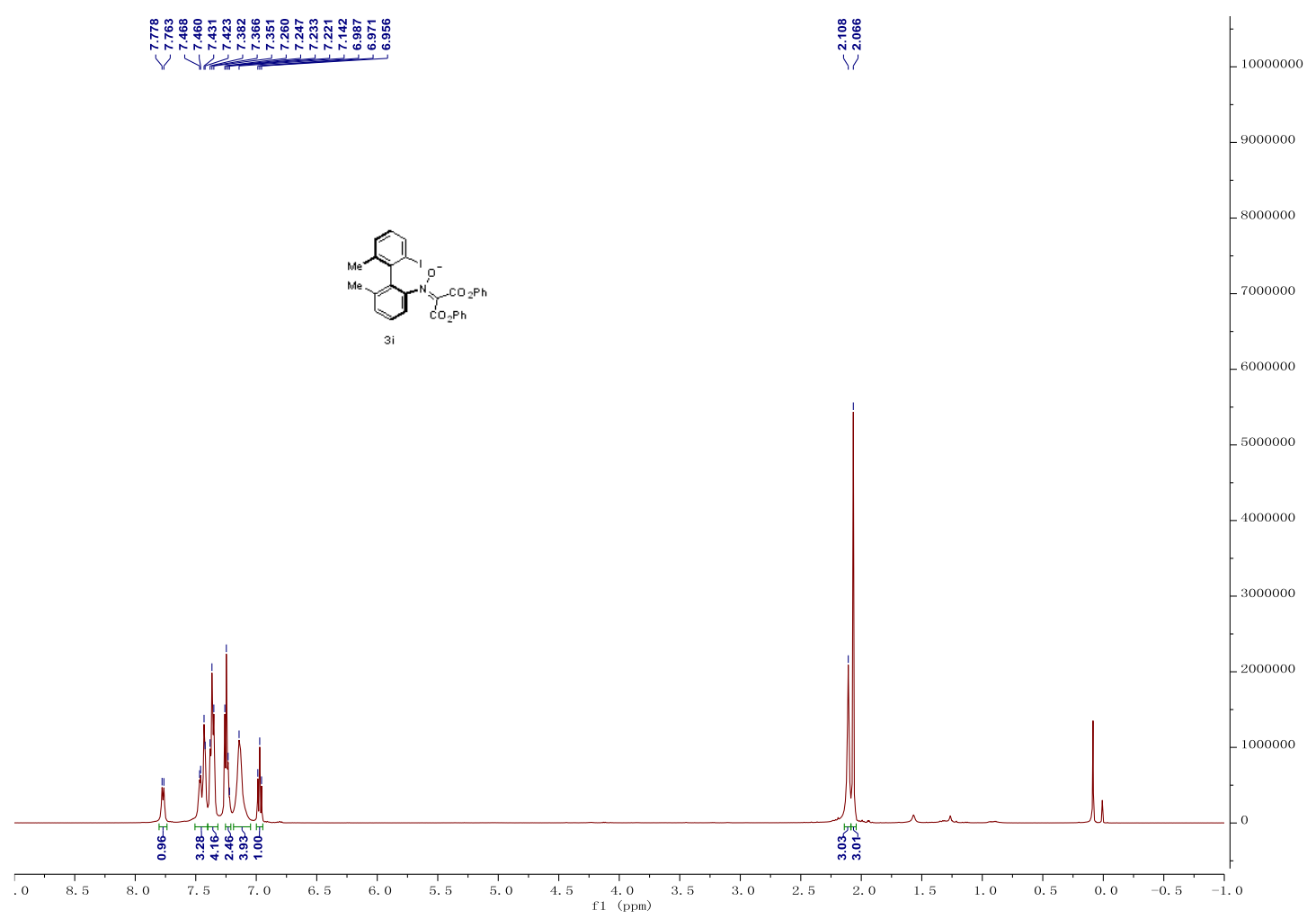

Figure S15. ${ }^{1} \mathrm{H}$ NMR spectra $\left(500 \mathrm{MHz}, \mathrm{CDCl}_{3}\right)$ of $\mathbf{3 i}$

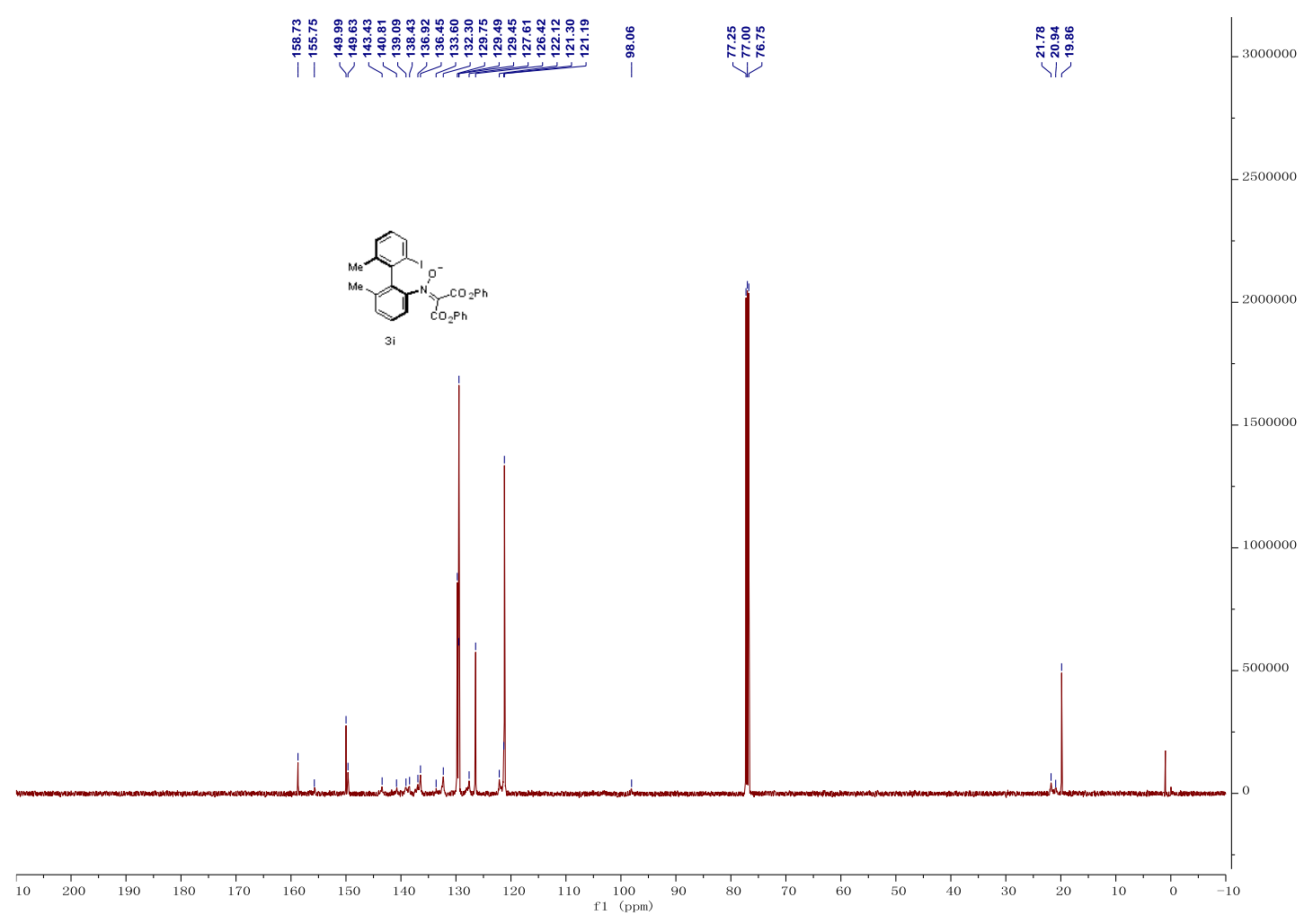

Figure S16. ${ }^{13} \mathrm{C}$ NMR spectra $\left(126 \mathrm{MHz}, \mathrm{CDCl}_{3}\right)$ of $\mathbf{3 i}$ 


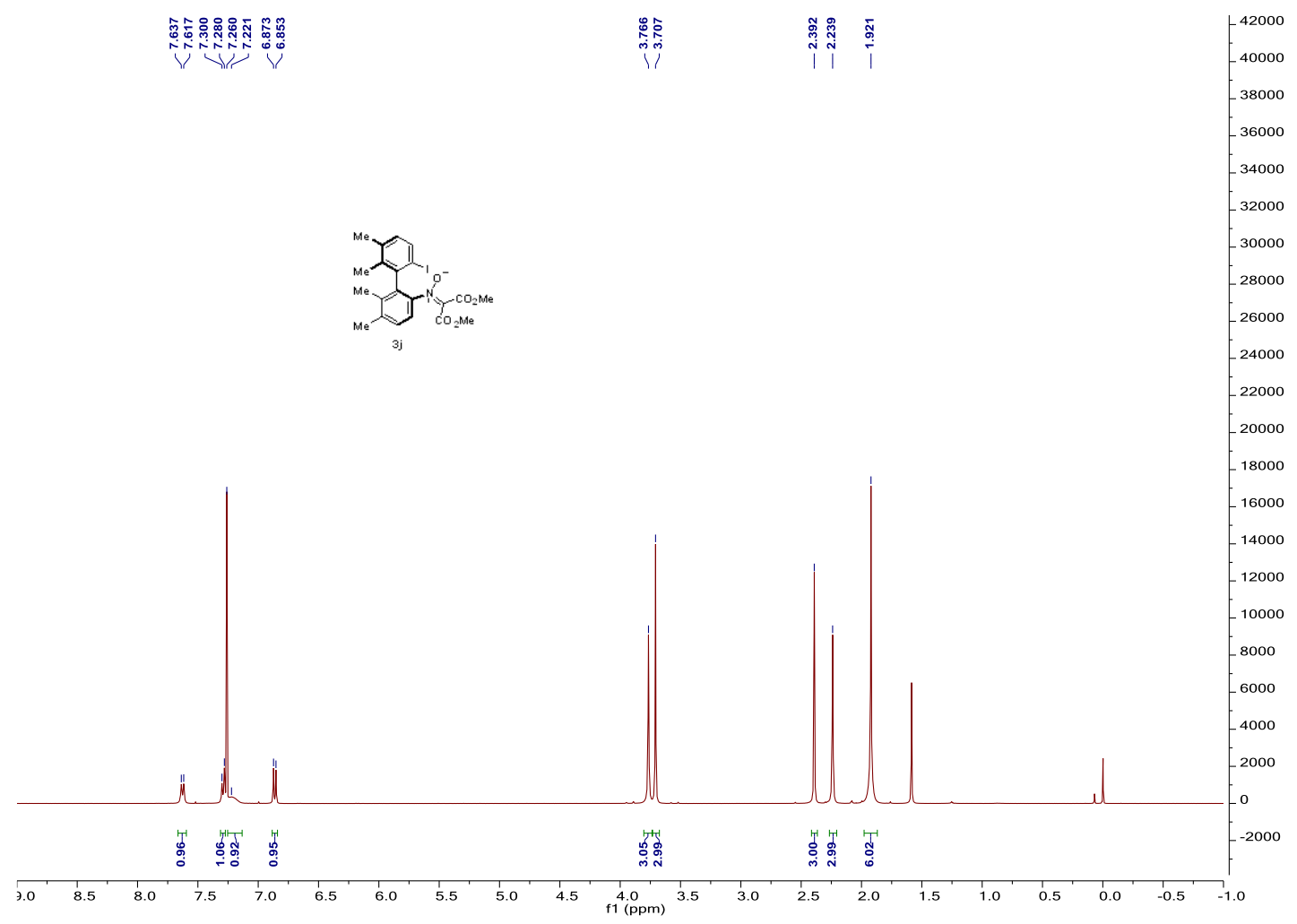

Figure S17. ${ }^{1} \mathrm{H}$ NMR spectra $\left(400 \mathrm{MHz}, \mathrm{CDCl}_{3}\right)$ of $\mathbf{3 j}$

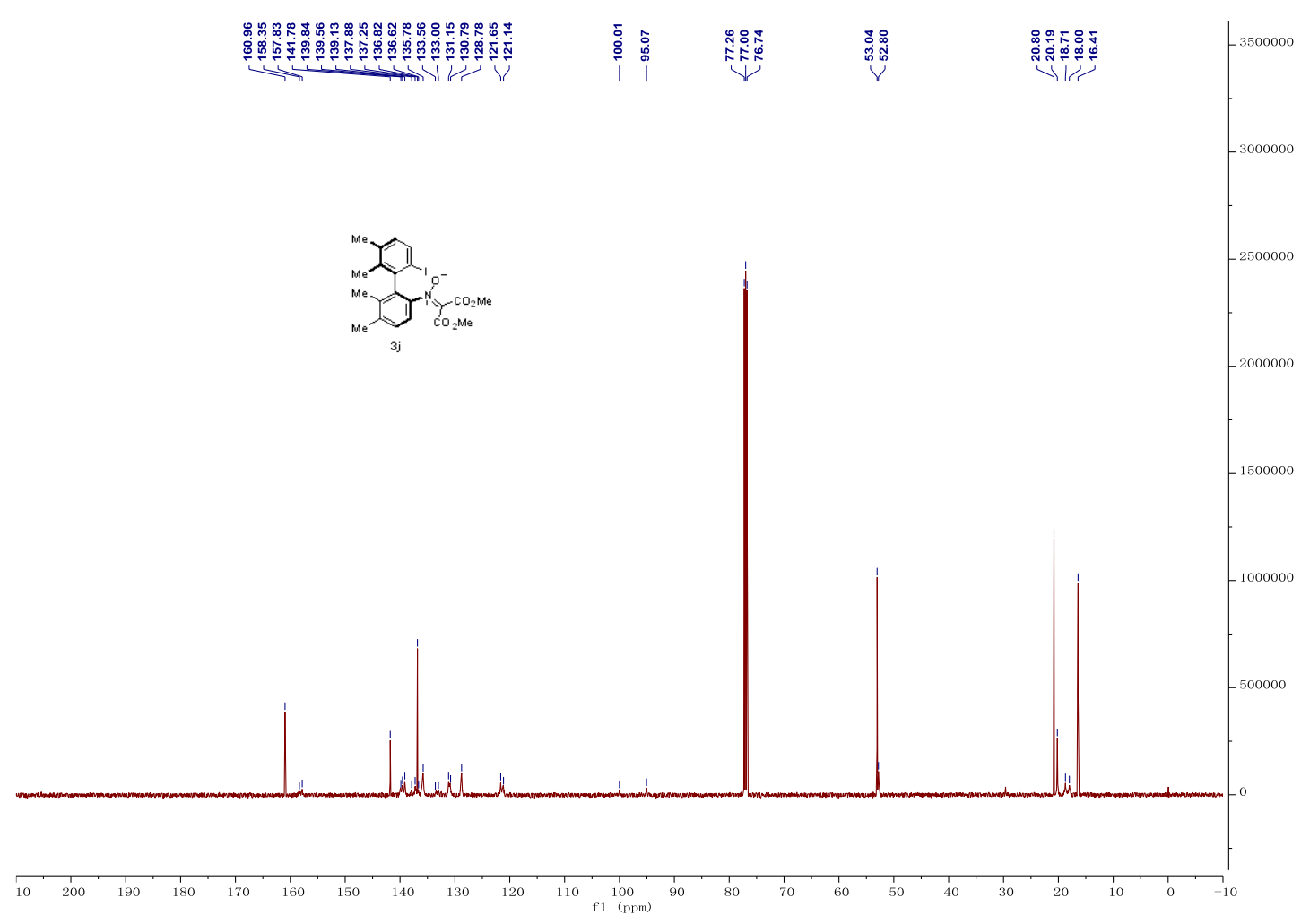

Figure S18. ${ }^{13} \mathrm{C}$ NMR spectra $\left(126 \mathrm{MHz}, \mathrm{CDCl}_{3}\right)$ of $\mathbf{3 j}$ 


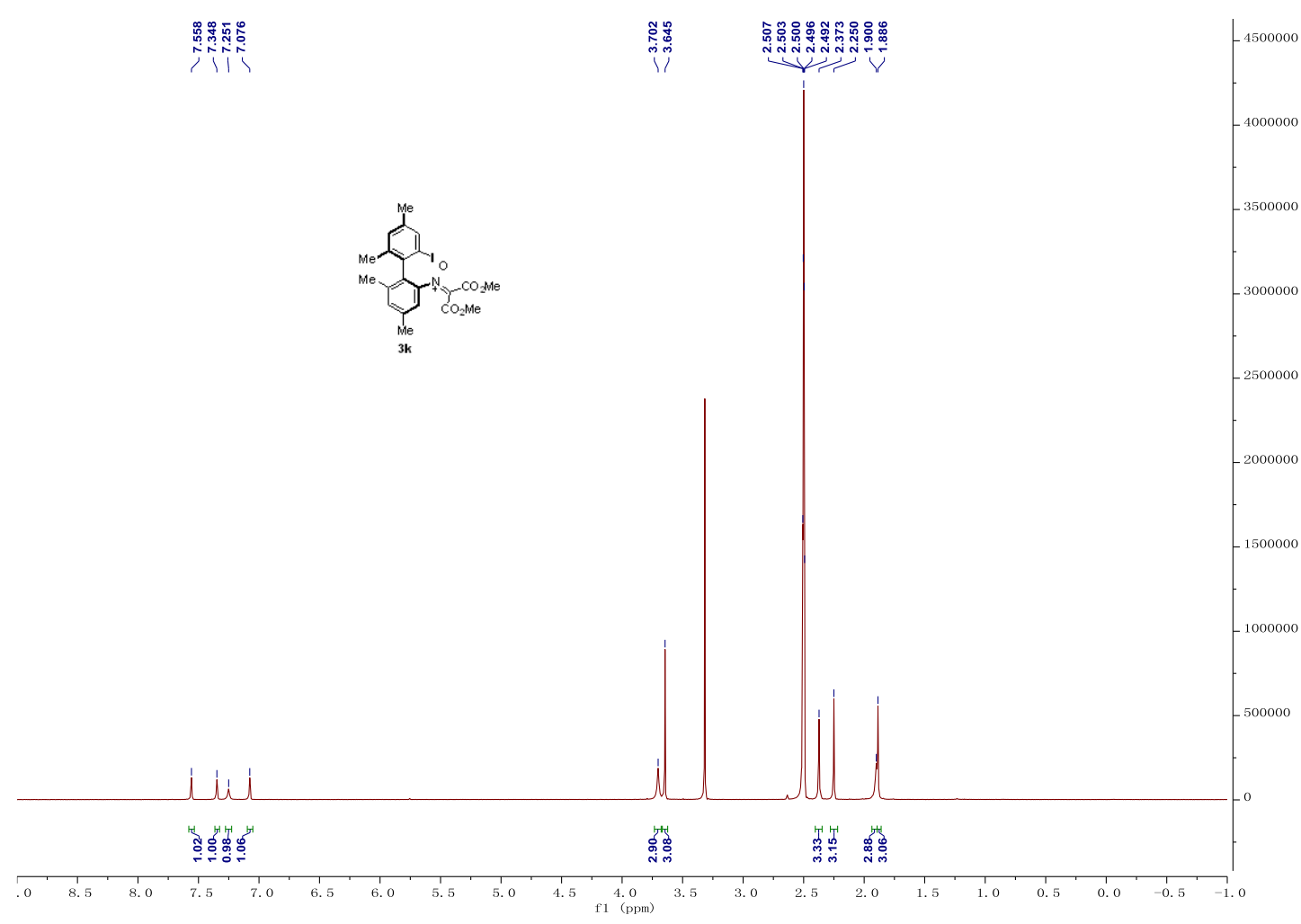

Figure S19. ${ }^{1} \mathrm{H}$ NMR spectra $\left(500 \mathrm{MHz}, \mathrm{DMSO}-d_{6}\right)$ of $\mathbf{3 k}$

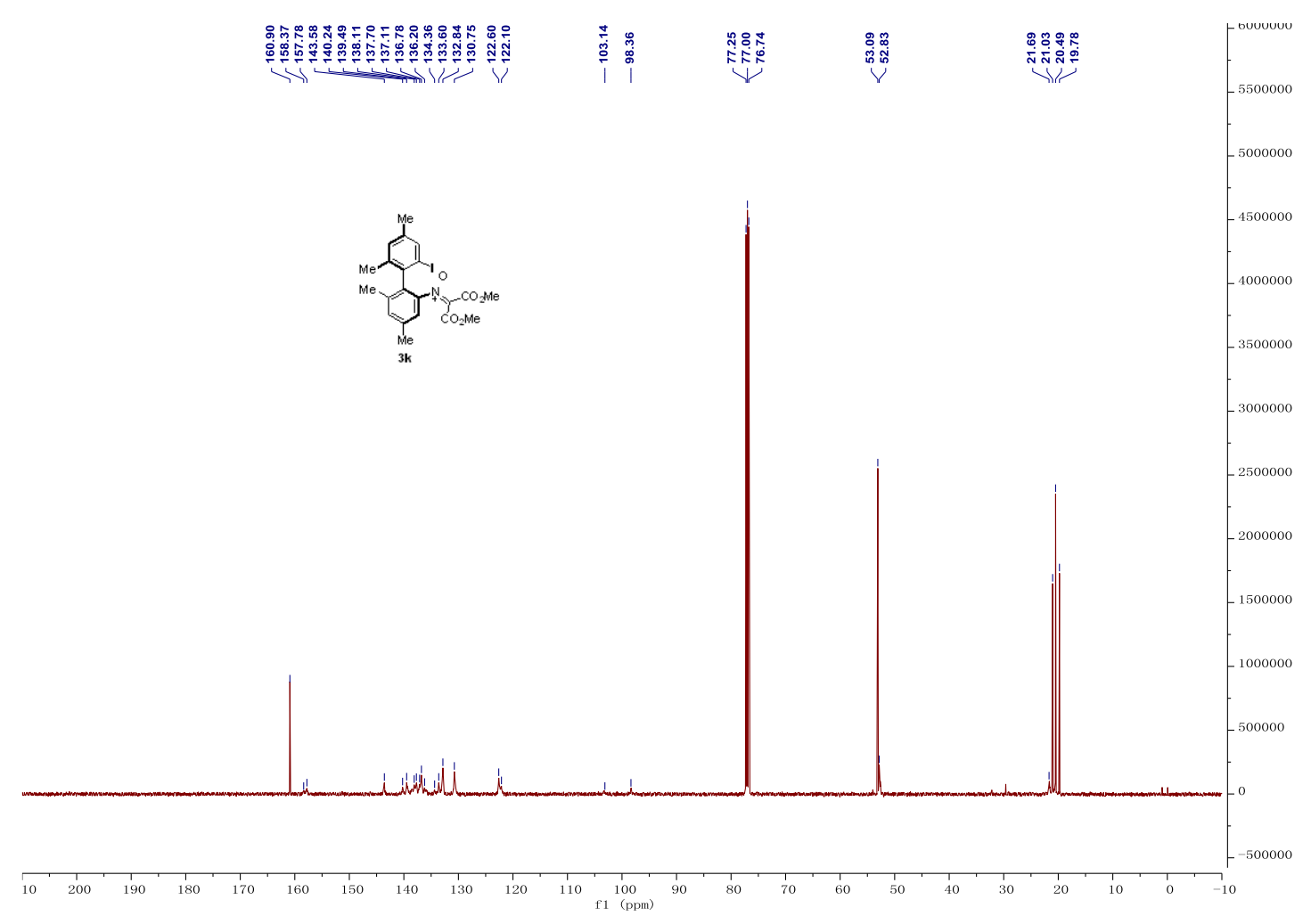

Figure S20. ${ }^{13} \mathrm{C}$ NMR spectra $\left(126 \mathrm{MHz}, \mathrm{CDCl}_{3}\right)$ of $\mathbf{3 k}$ 


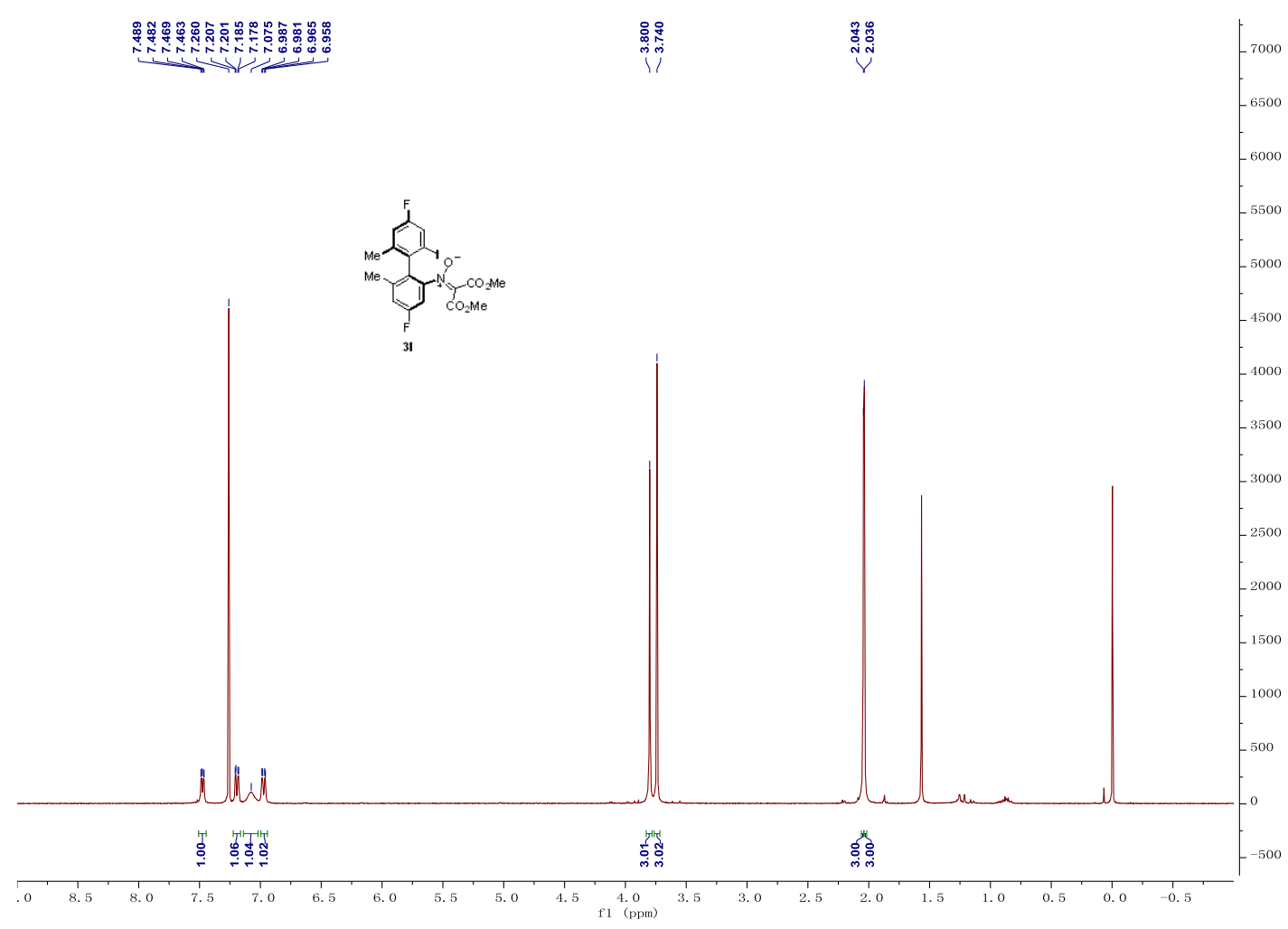

Figure S21. ${ }^{1} \mathrm{H}$ NMR spectra $\left(400 \mathrm{MHz}, \mathrm{CDCl}_{3}\right)$ of $\mathbf{3 l}$

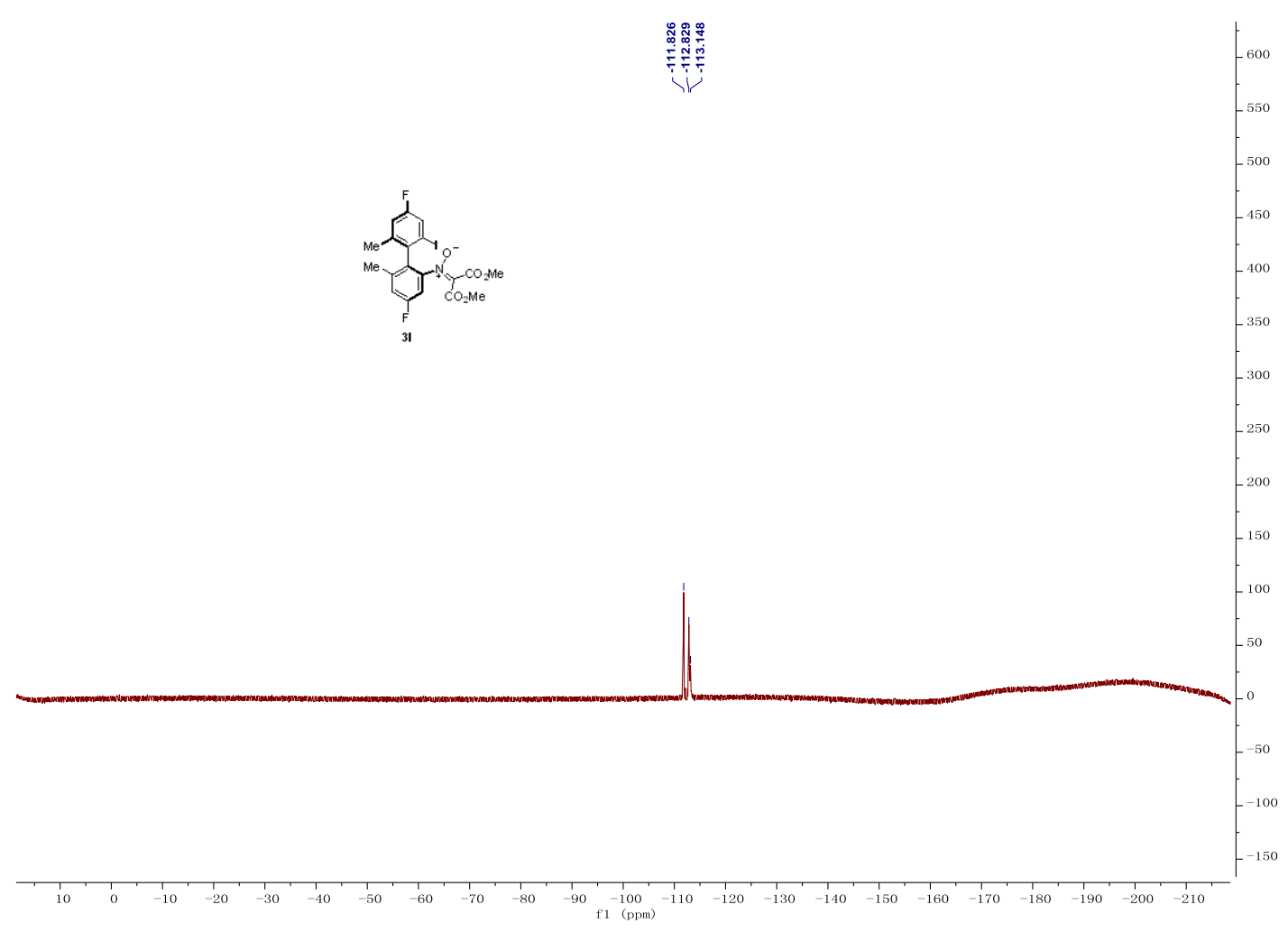

Figure S22. ${ }^{19} \mathrm{~F}$ NMR spectra $\left(376 \mathrm{MHz}, \mathrm{CDCl}_{3}\right)$ of $\mathbf{3 l}$ 


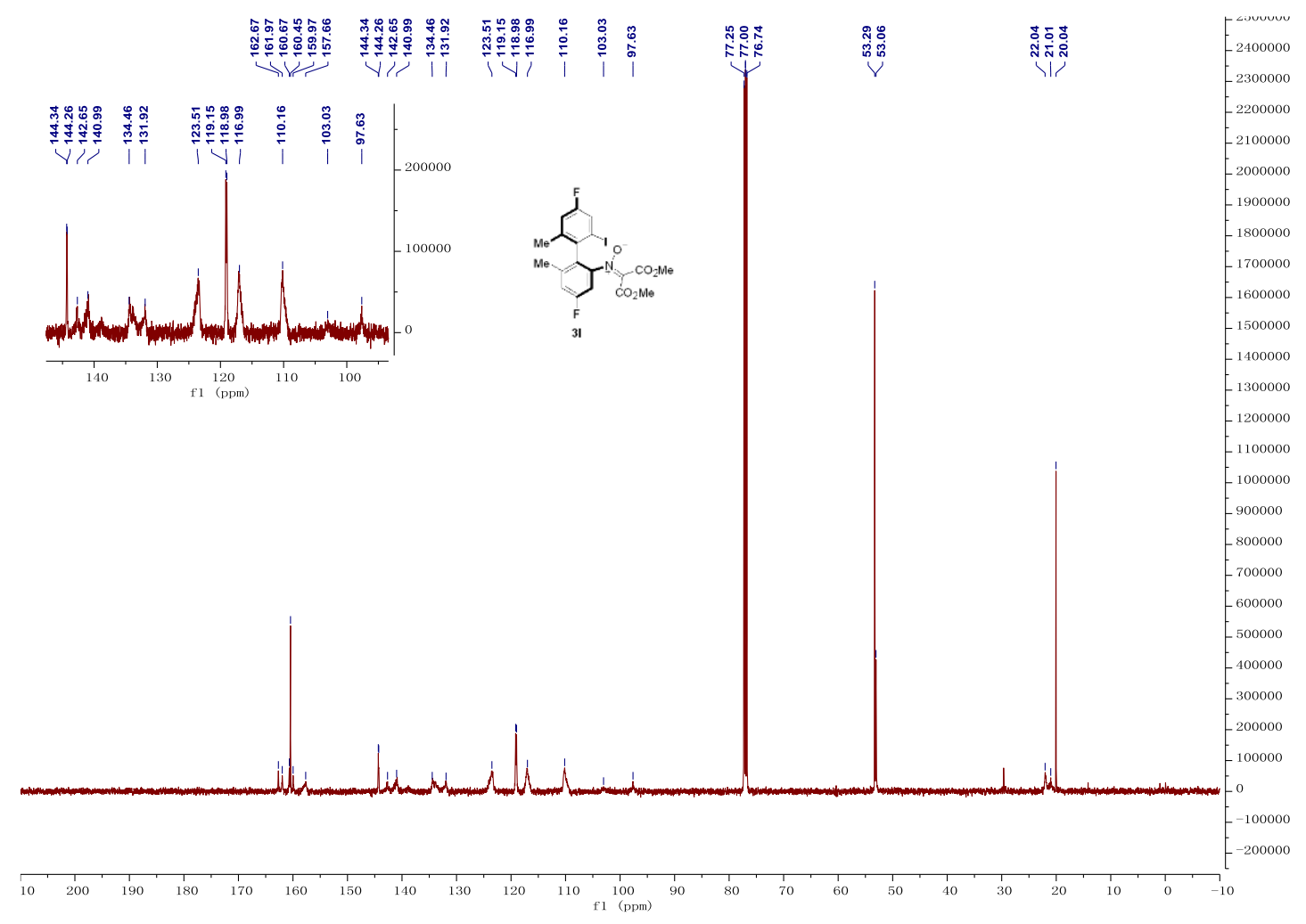

Figure S23. ${ }^{13} \mathrm{C}$ NMR spectra $\left(126 \mathrm{MHz}, \mathrm{CDCl}_{3}\right)$ of $\mathbf{3 l}$

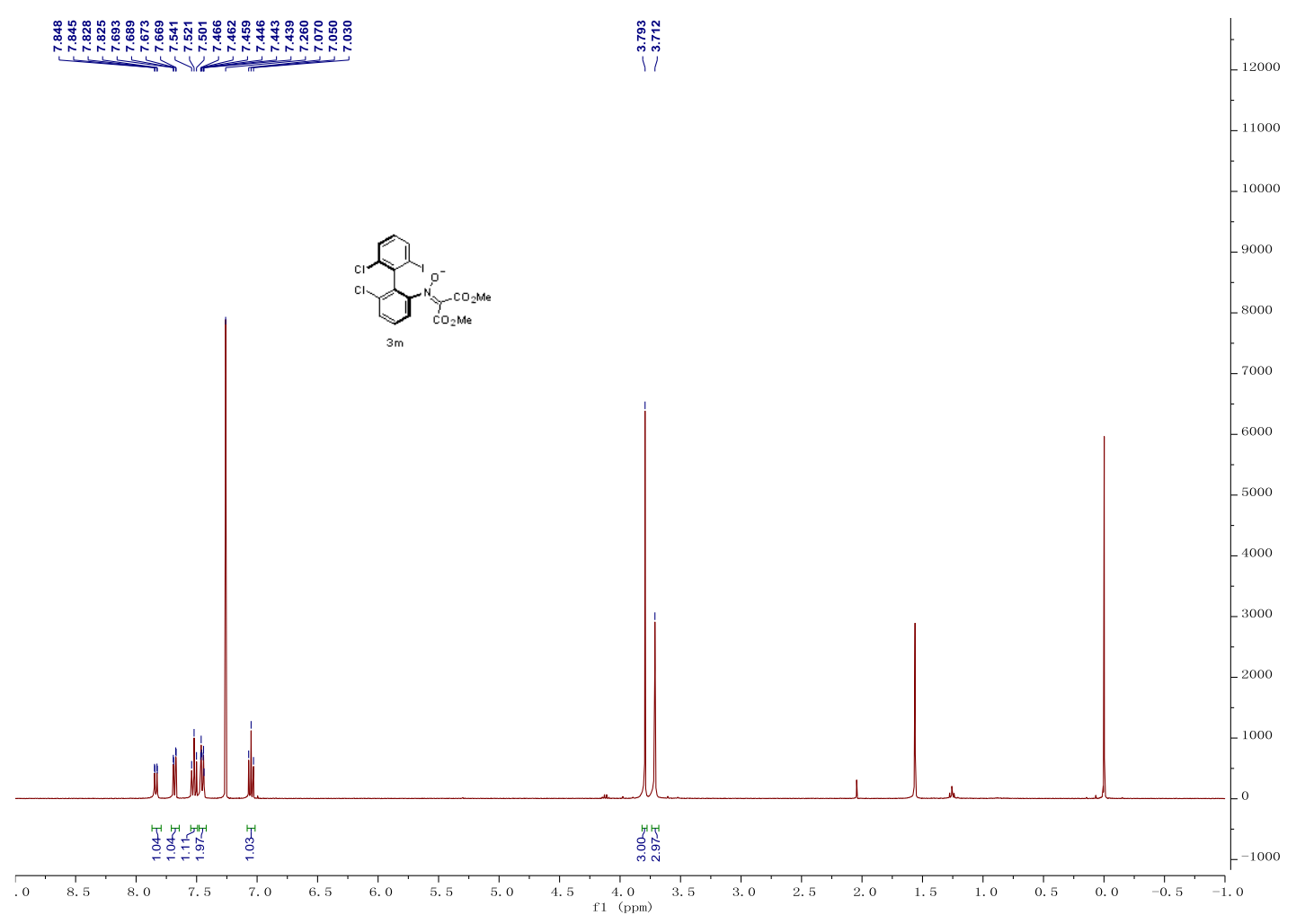

Figure S24. ${ }^{1} \mathrm{H}$ NMR spectra $\left(400 \mathrm{MHz}, \mathrm{CDCl}_{3}\right)$ of $\mathbf{3 m}$ 


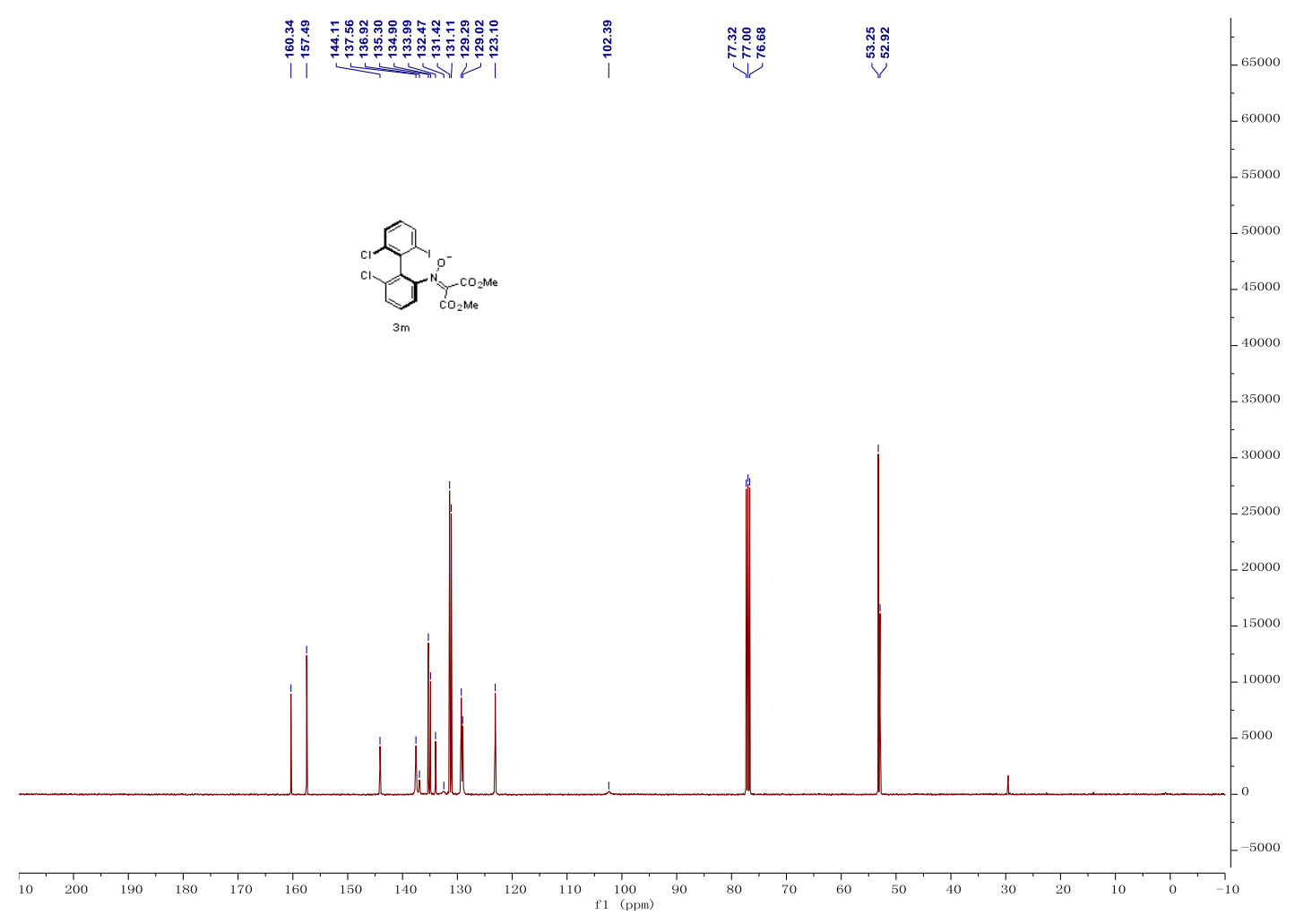

Figure S25. ${ }^{13} \mathrm{C}$ NMR spectra $\left(101 \mathrm{MHz}, \mathrm{CDCl}_{3}\right)$ of $\mathbf{3 m}$

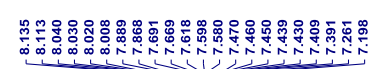
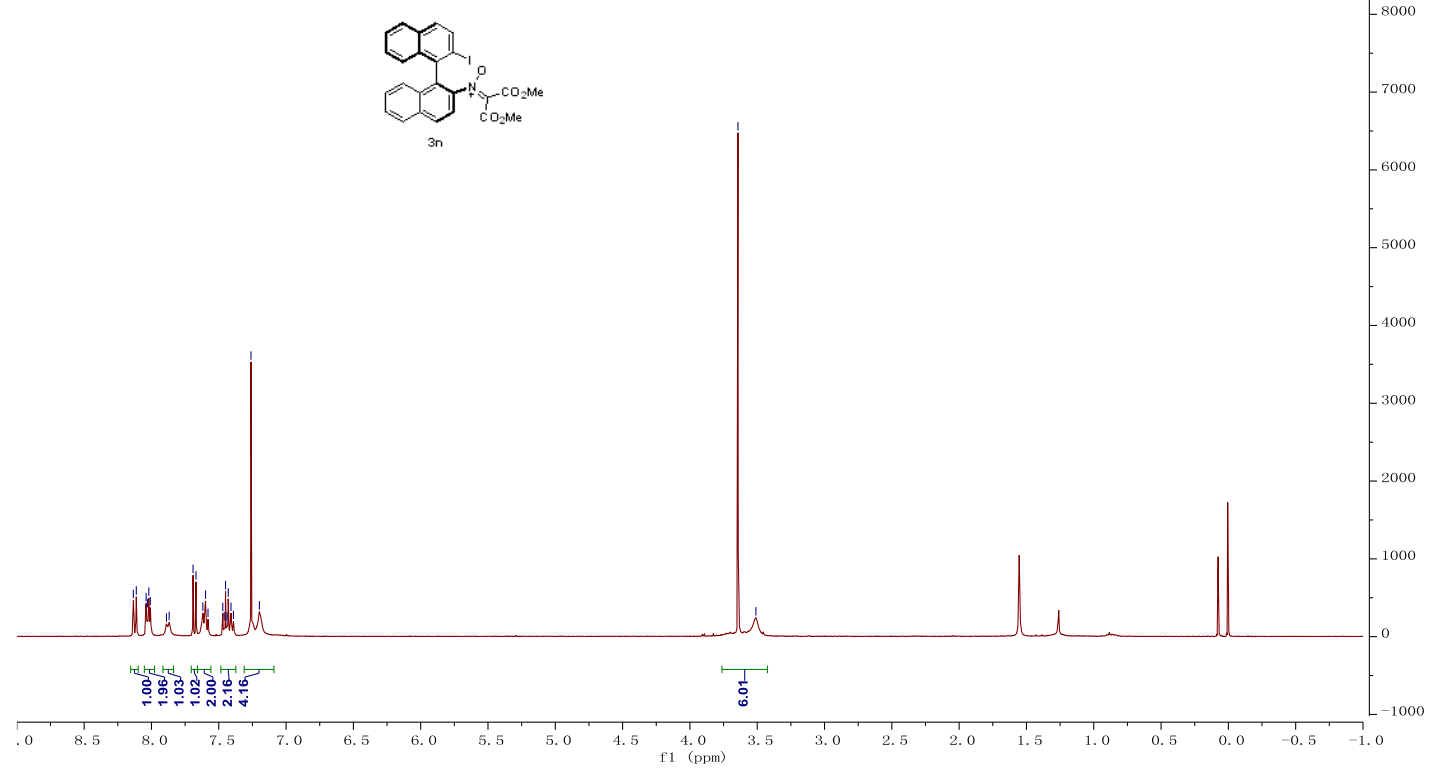

Figure S26. ${ }^{1} \mathrm{H}$ NMR spectra $\left(400 \mathrm{MHz}, \mathrm{CDCl}_{3}\right)$ of $\mathbf{3 n}$ 


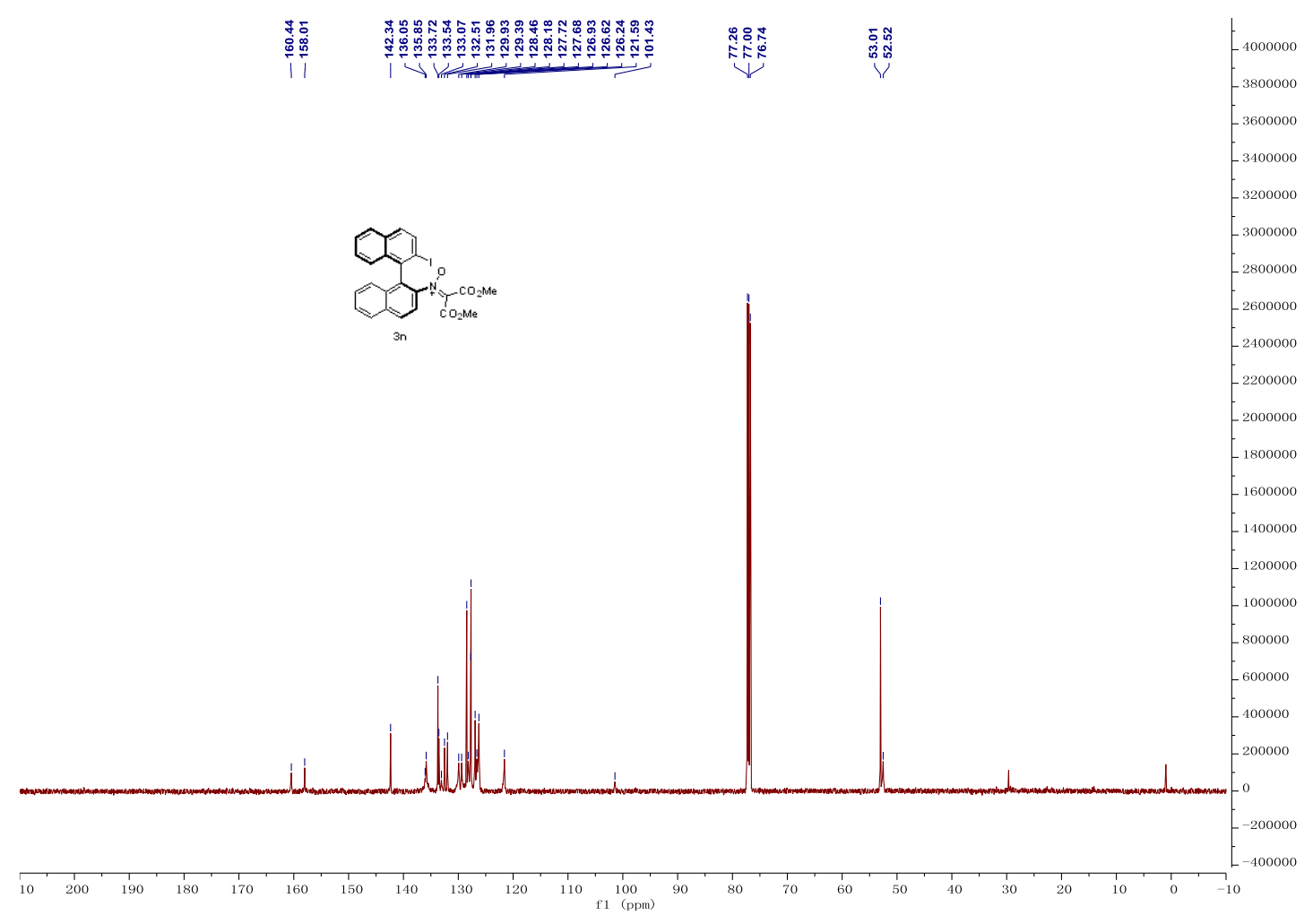

Figure S27. ${ }^{13} \mathrm{C}$ NMR spectra $\left(126 \mathrm{MHz}, \mathrm{CDCl}_{3}\right)$ of $\mathbf{3 n}$

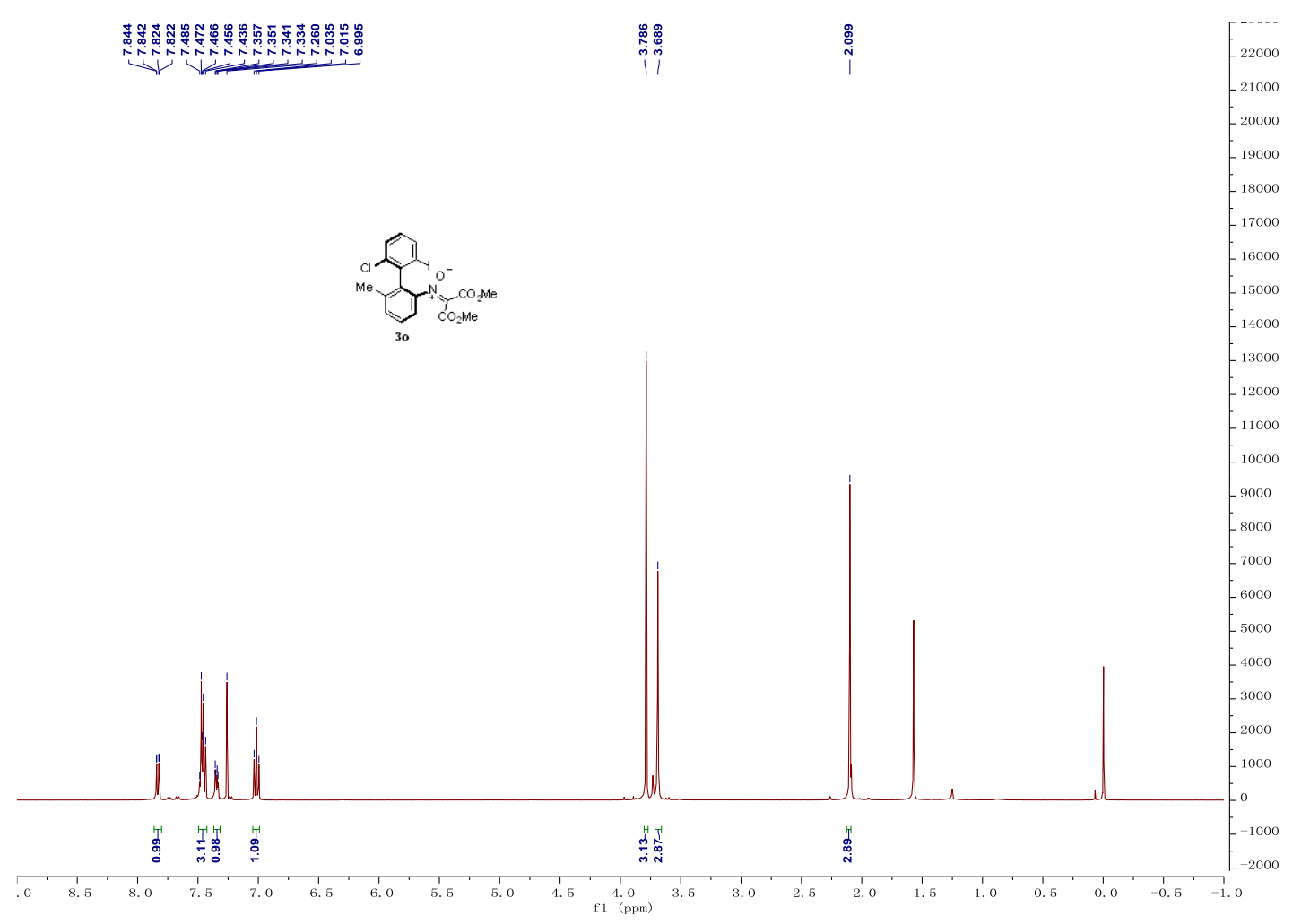

Figure S28. ${ }^{1} \mathrm{H}$ NMR spectra $\left(400 \mathrm{MHz}, \mathrm{CDCl}_{3}\right)$ of 30 


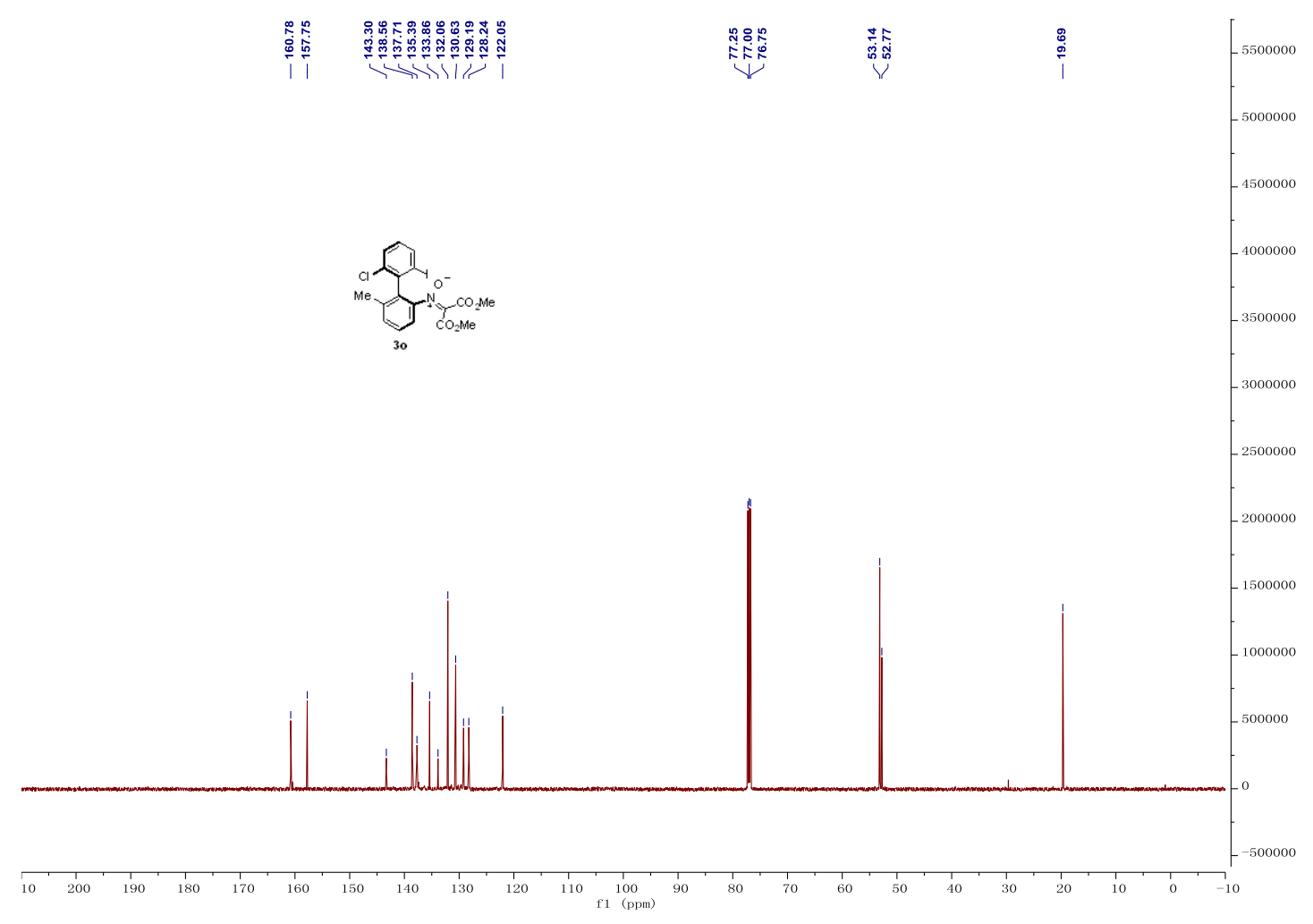

Figure S29. ${ }^{13} \mathrm{C}$ NMR spectra $\left(126 \mathrm{MHz}, \mathrm{CDCl}_{3}\right)$ of $\mathbf{3 o}$

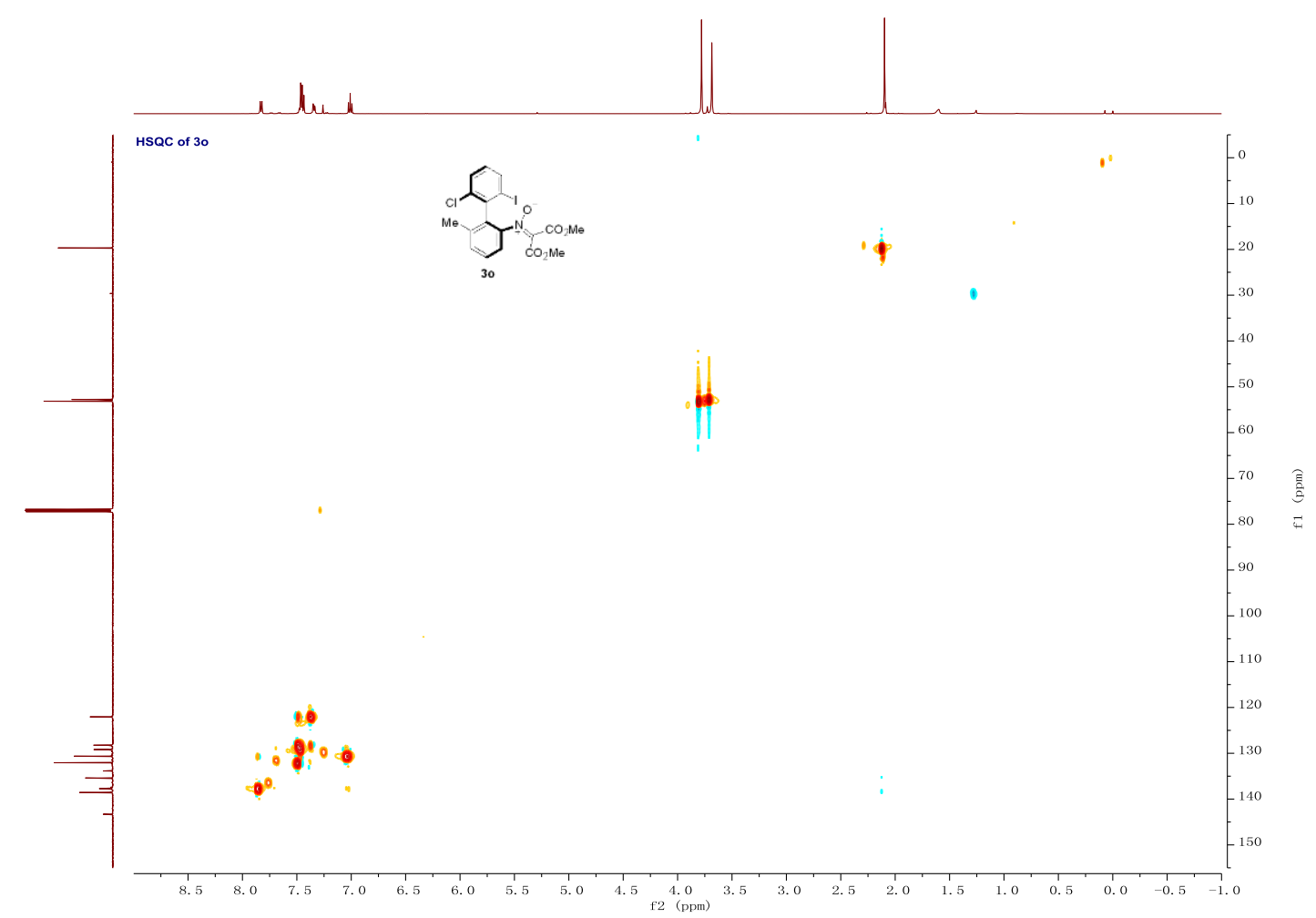

Figure S30. HSQC NMR spectra (400 MHz, $\left.101 \mathrm{MHz}, \mathrm{CDCl}_{3}\right)$ of $\mathbf{3 o}$ 


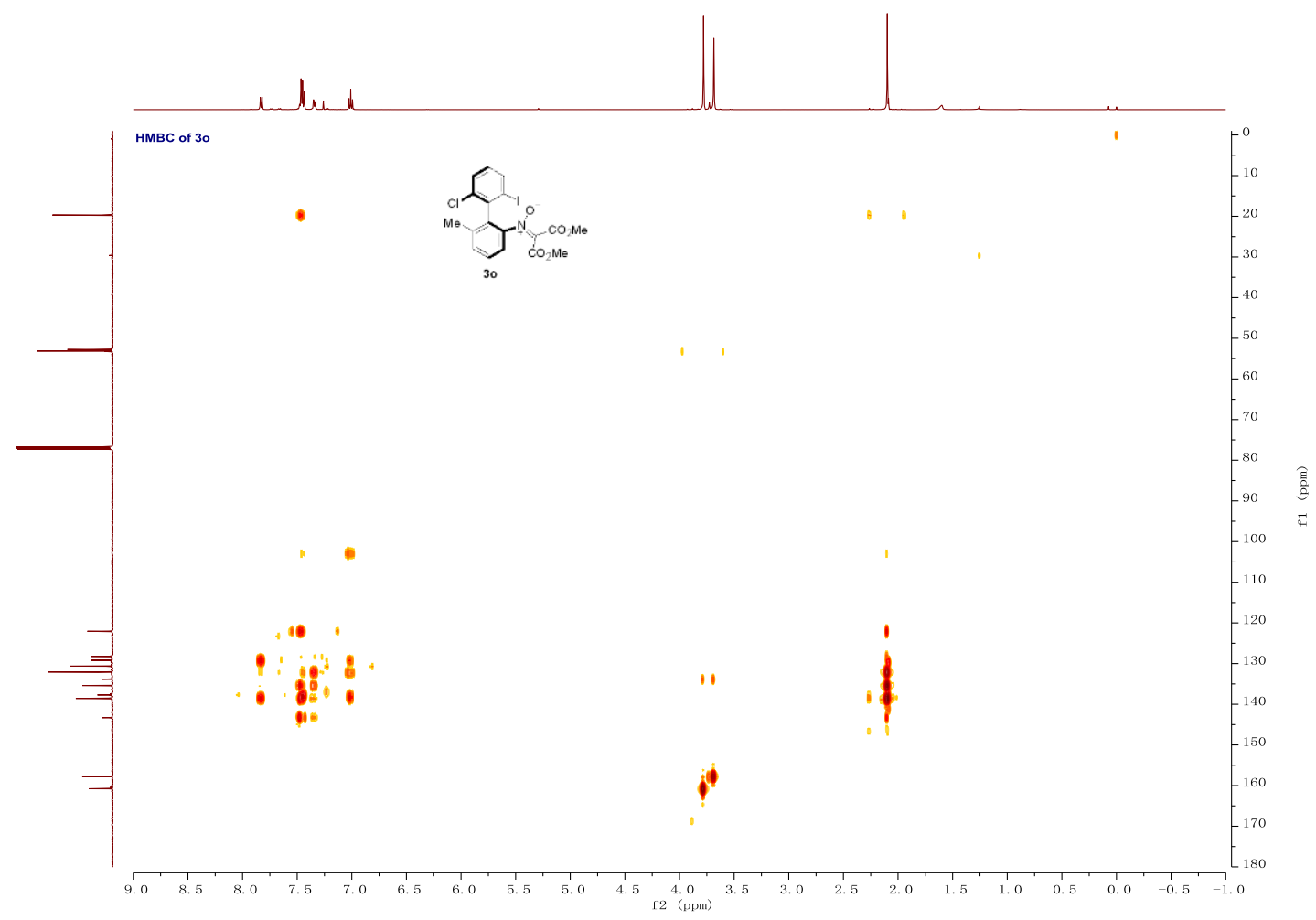

Figure S31. HMBC NMR spectra (400 MHz, $\left.101 \mathrm{MHz}, \mathrm{CDCl}_{3}\right)$ of $\mathbf{3 o}$

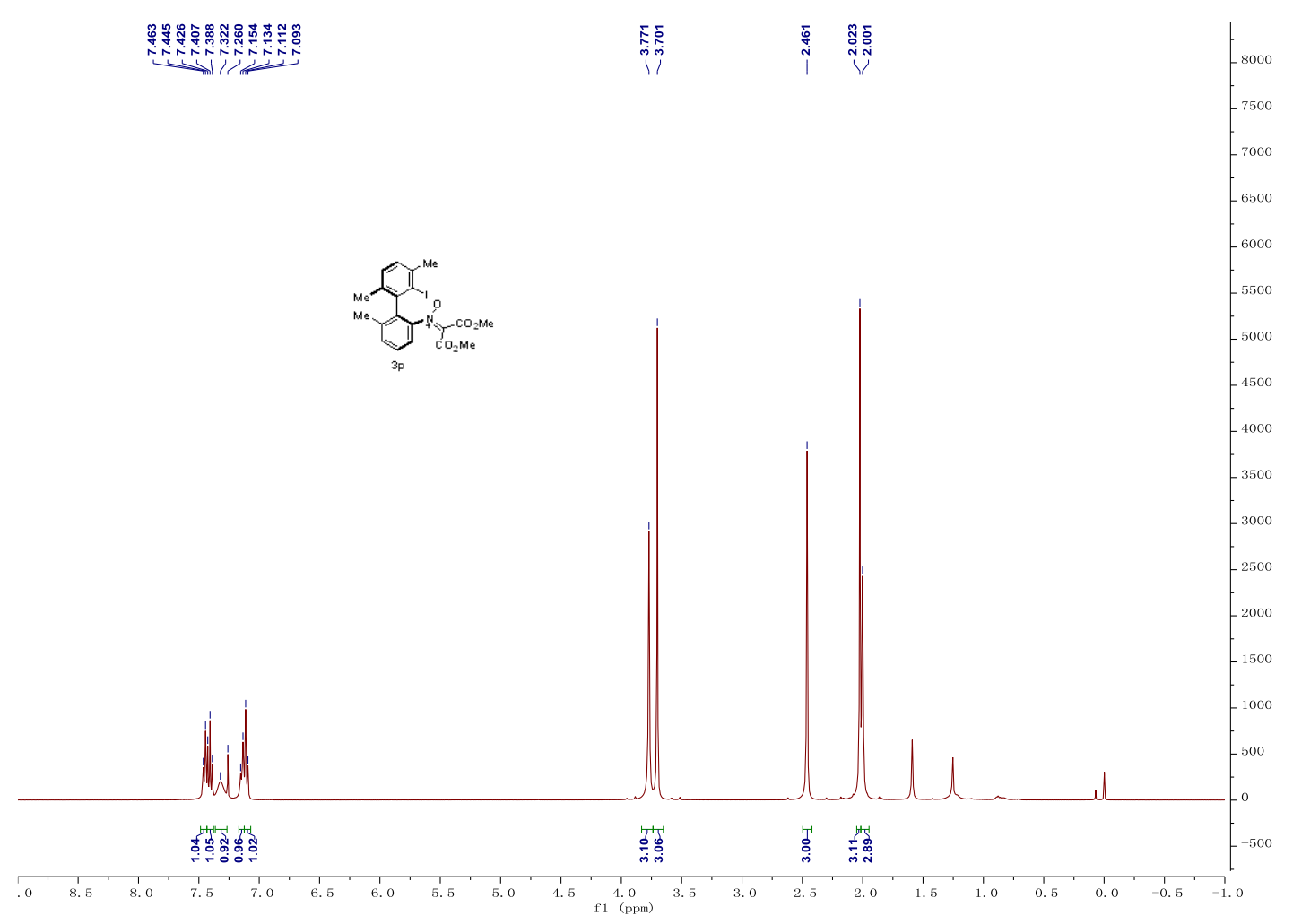

Figure S32. ${ }^{1} \mathrm{H}$ NMR spectra $\left(400 \mathrm{MHz}, \mathrm{CDCl}_{3}\right)$ of $\mathbf{3 p}$ 


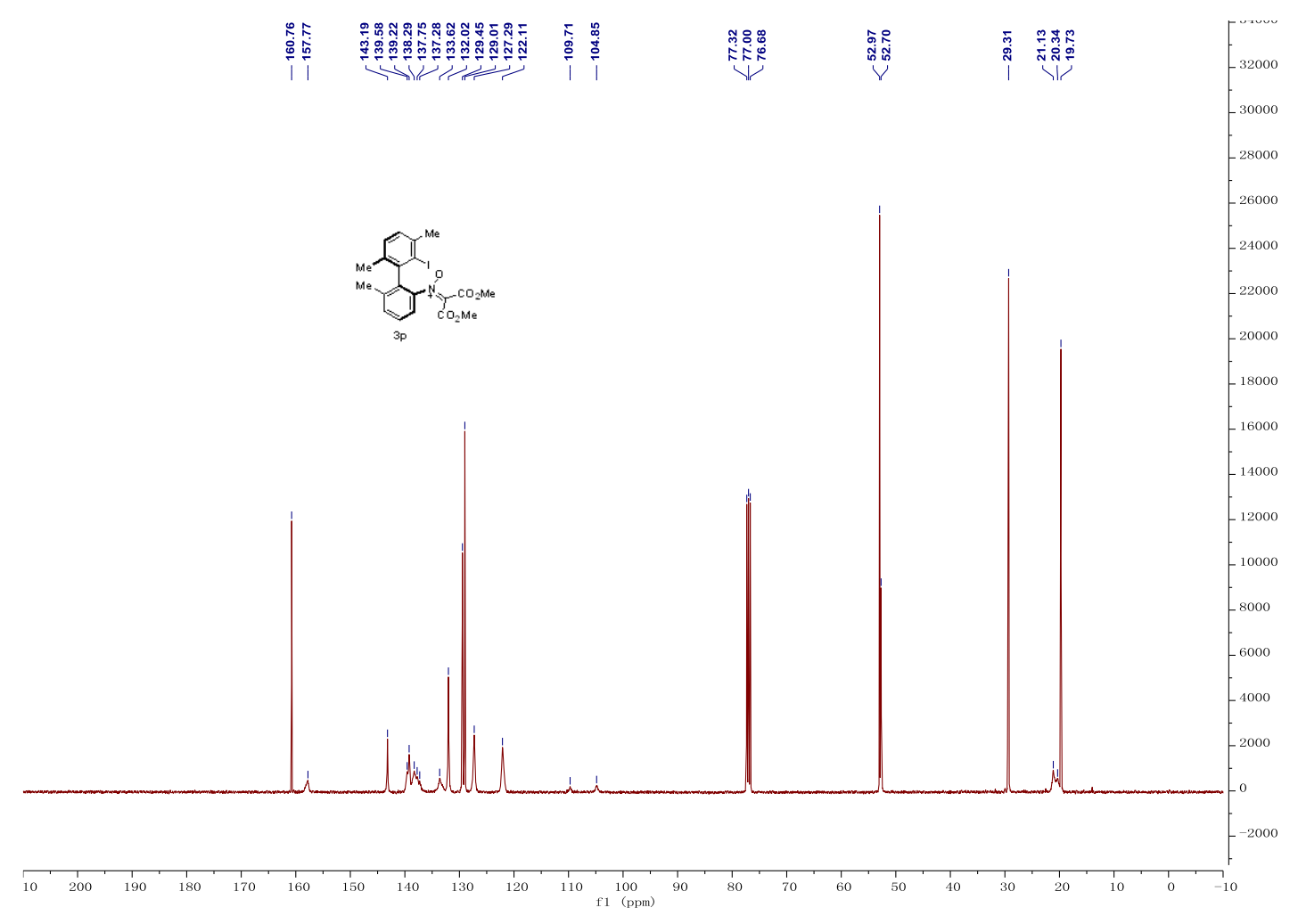

Figure S33. ${ }^{13} \mathrm{C}$ NMR spectra $\left(101 \mathrm{MHz}, \mathrm{CDCl}_{3}\right)$ of $\mathbf{3 p}$

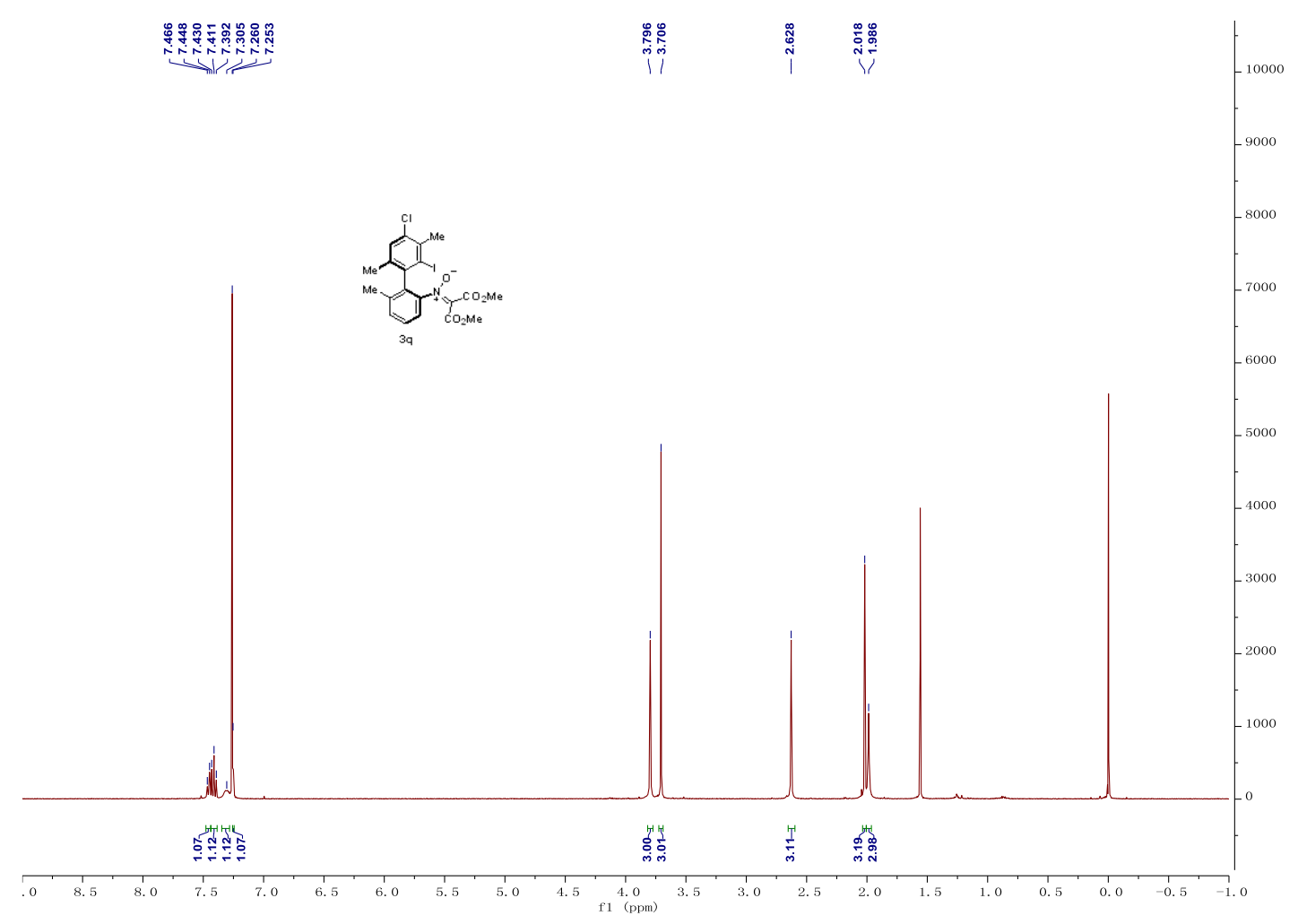

Figure S34. ${ }^{1} \mathrm{H}$ NMR spectra $\left(400 \mathrm{MHz}, \mathrm{CDCl}_{3}\right)$ of $\mathbf{3 q}$ 


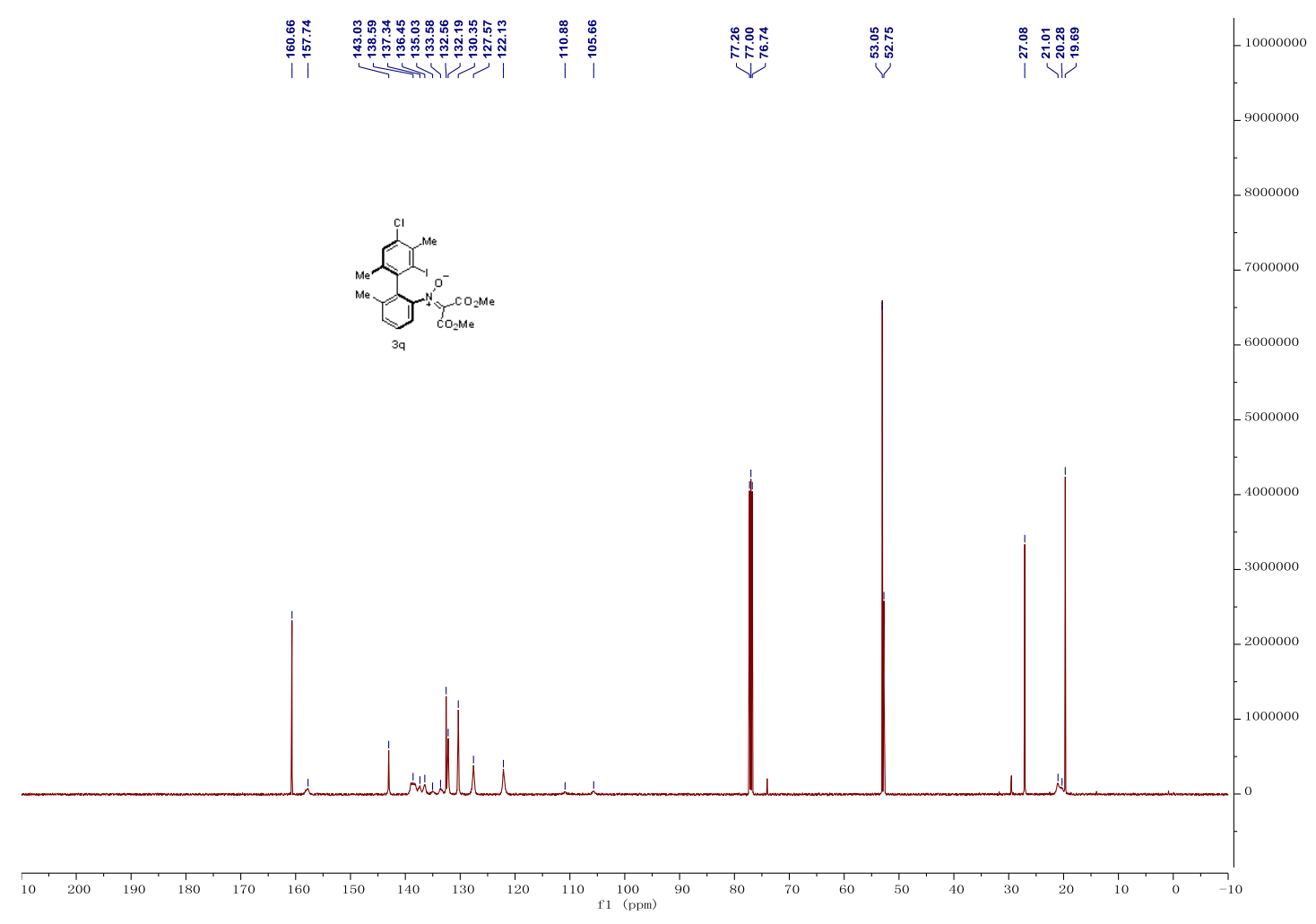

Figure S35. ${ }^{13} \mathrm{C}$ NMR spectra $\left(126 \mathrm{MHz}, \mathrm{CDCl}_{3}\right)$ of $\mathbf{3 q}$

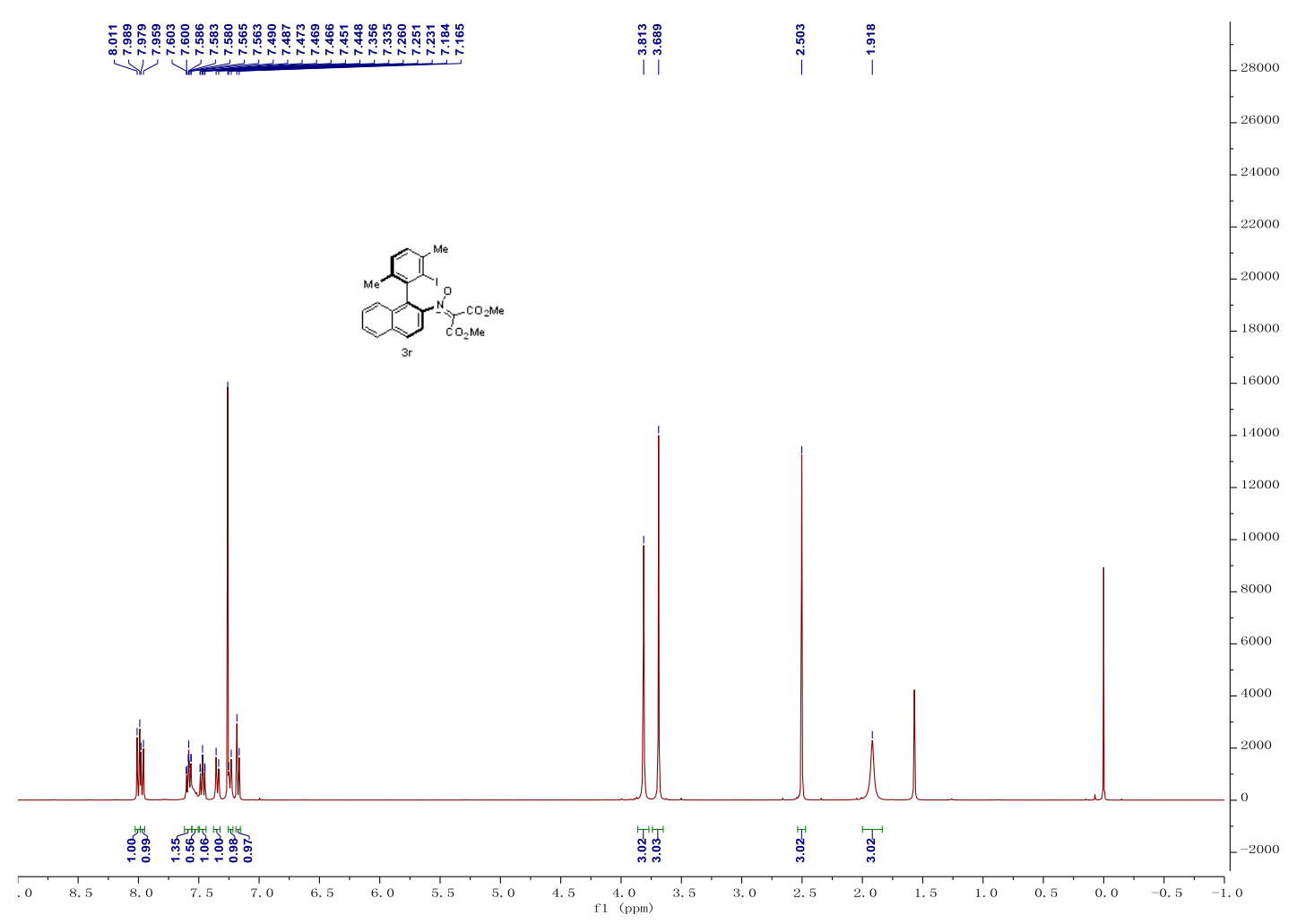

Figure S36. ${ }^{1} \mathrm{H}$ NMR spectra $\left(400 \mathrm{MHz}, \mathrm{CDCl}_{3}\right)$ of $\mathbf{3 r}$ 


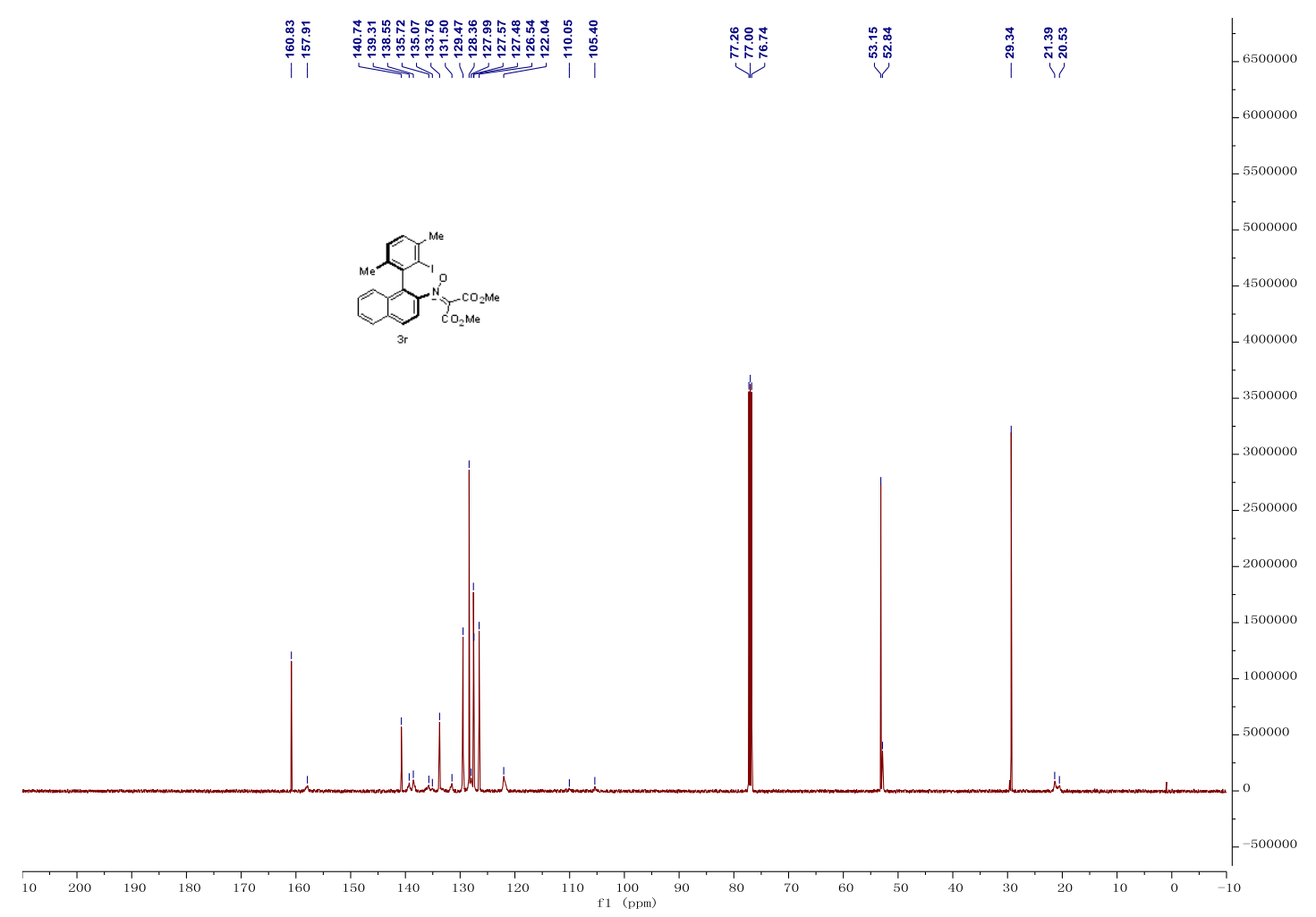

Figure S37. ${ }^{13} \mathrm{C}$ NMR spectra $\left(126 \mathrm{MHz}, \mathrm{CDCl}_{3}\right)$ of $\mathbf{3 r}$

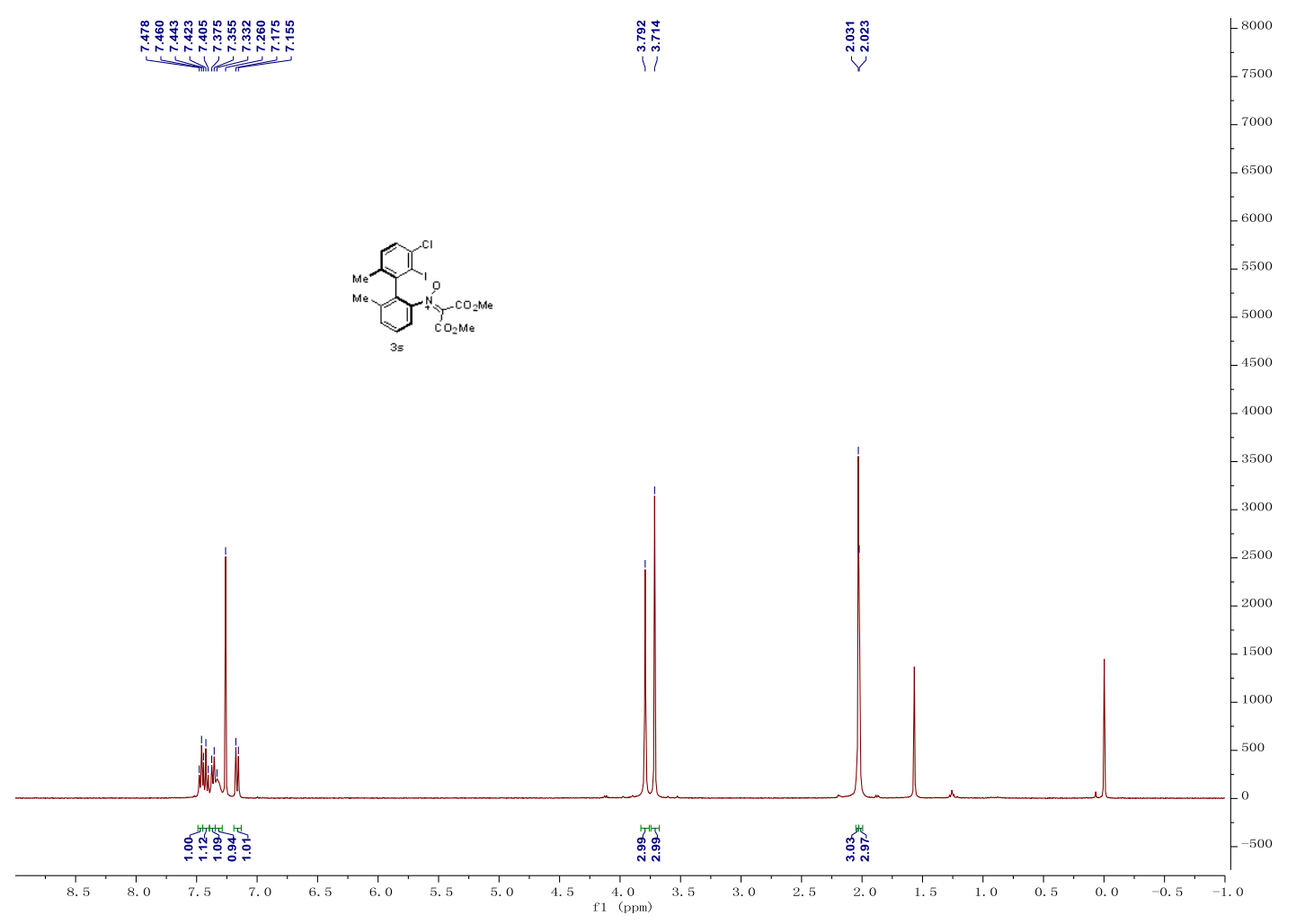

Figure S38. ${ }^{1} \mathrm{H}$ NMR spectra $\left(400 \mathrm{MHz}, \mathrm{CDCl}_{3}\right)$ of $\mathbf{3 s}$ 


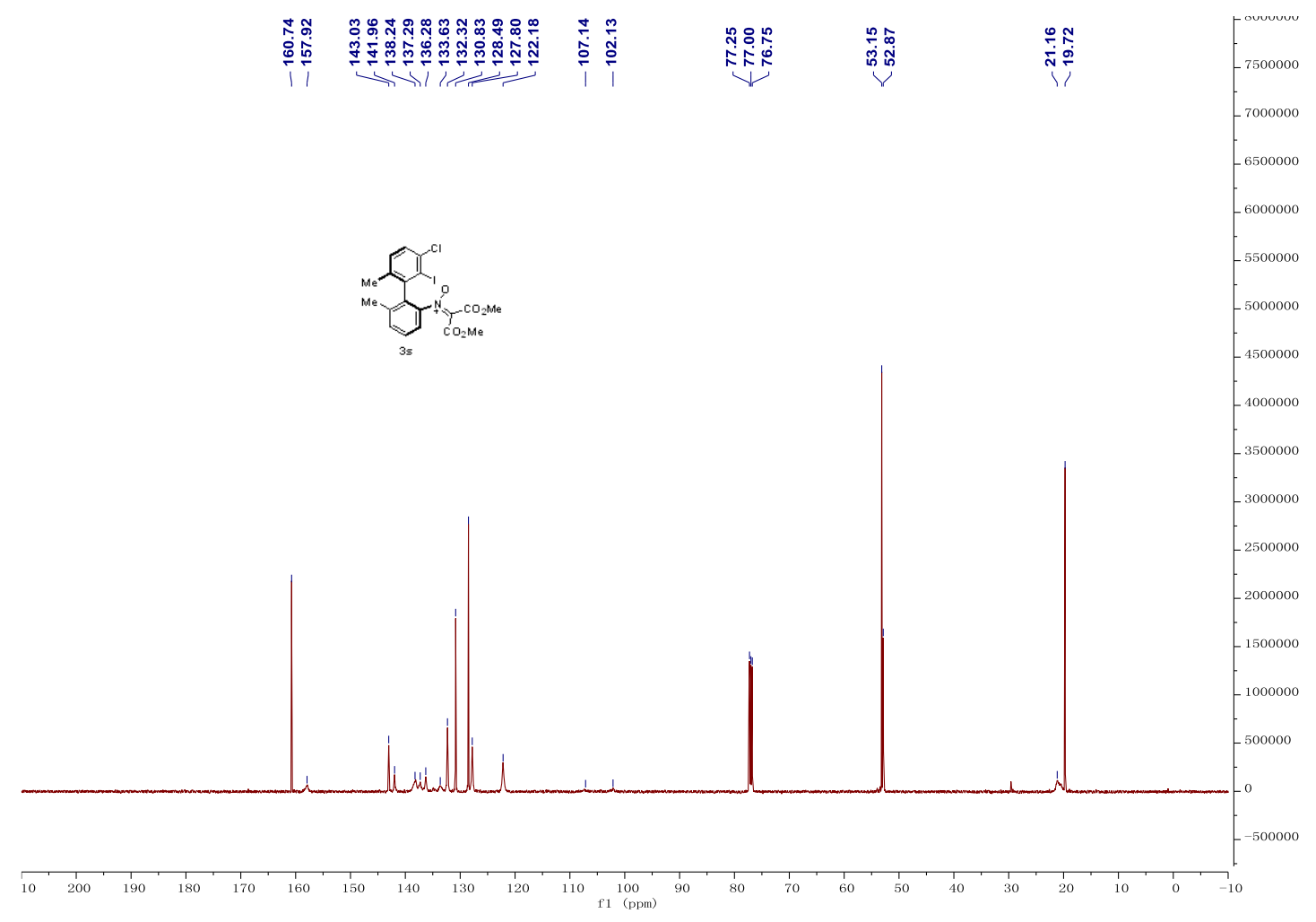

Figure S39. ${ }^{13} \mathrm{C}$ NMR spectra $\left(126 \mathrm{MHz}, \mathrm{CDCl}_{3}\right)$ of $3 \mathbf{s}$

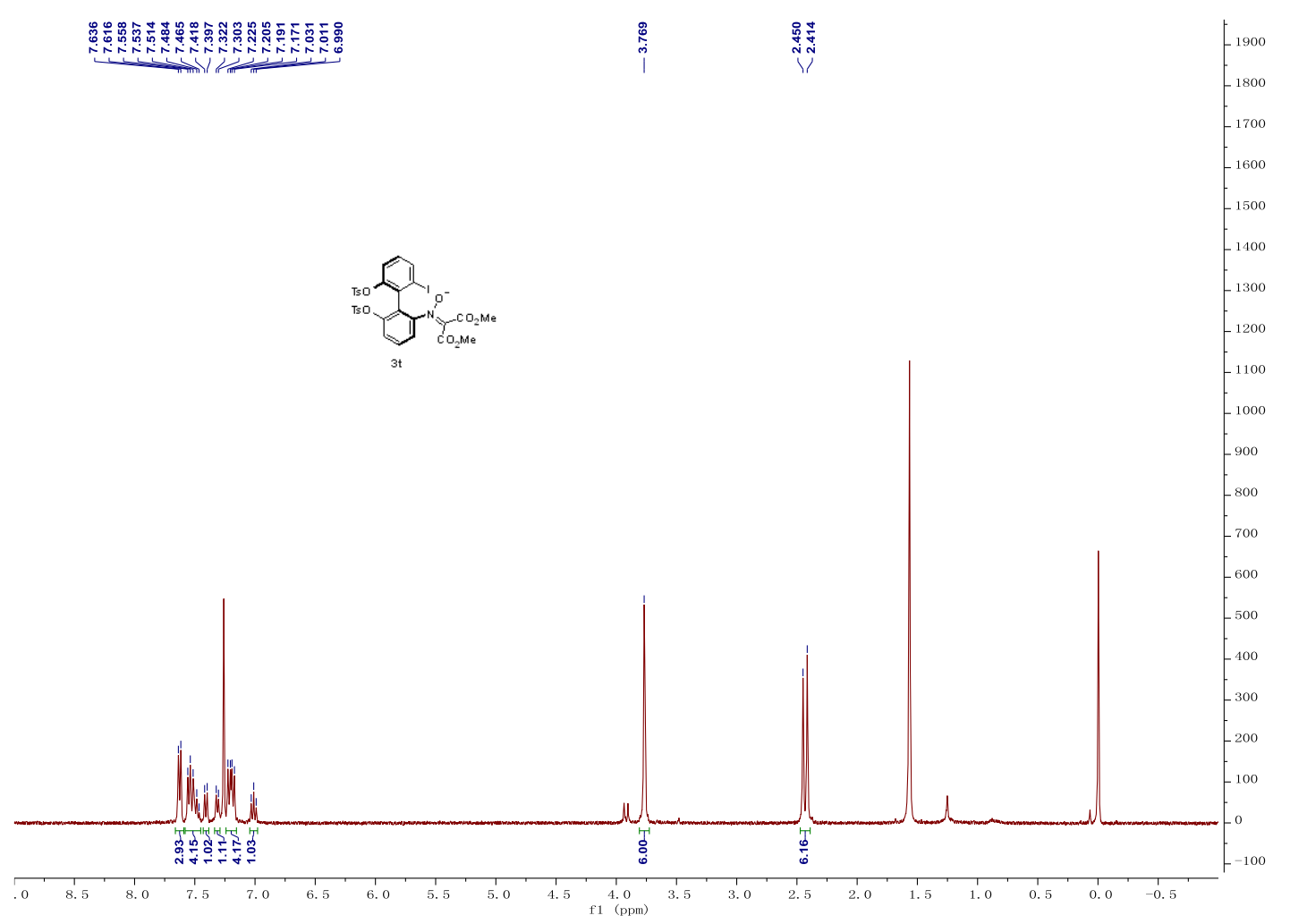

Figure S40. ${ }^{1} \mathrm{H}$ NMR spectra (400 $\mathrm{MHz}, \mathrm{CDCl}_{3}$ ) of $\mathbf{3 t}$ 


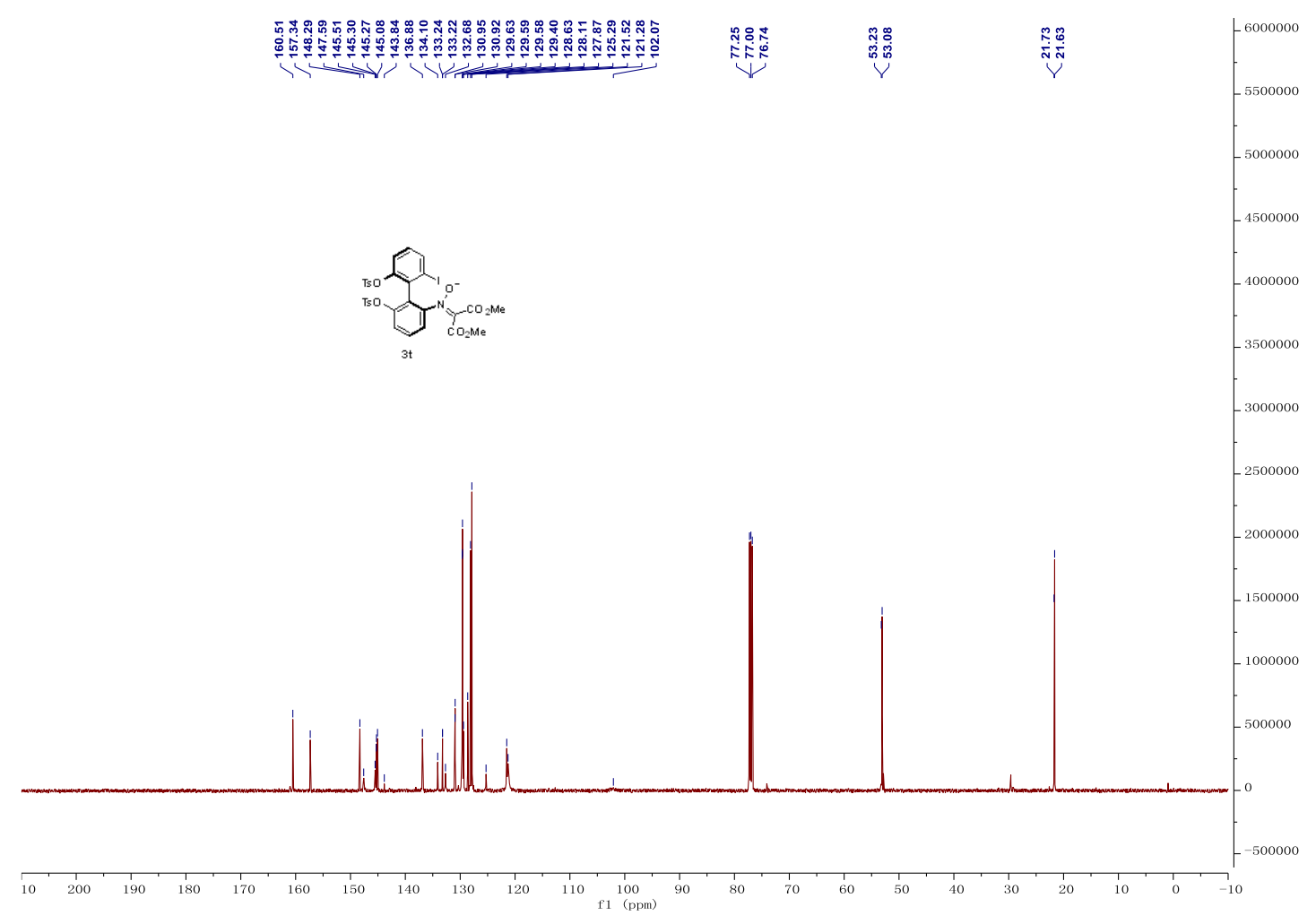

Figure S41. ${ }^{13} \mathrm{C}$ NMR spectra $\left(126 \mathrm{MHz}, \mathrm{CDCl}_{3}\right)$ of $\mathbf{3 t}$

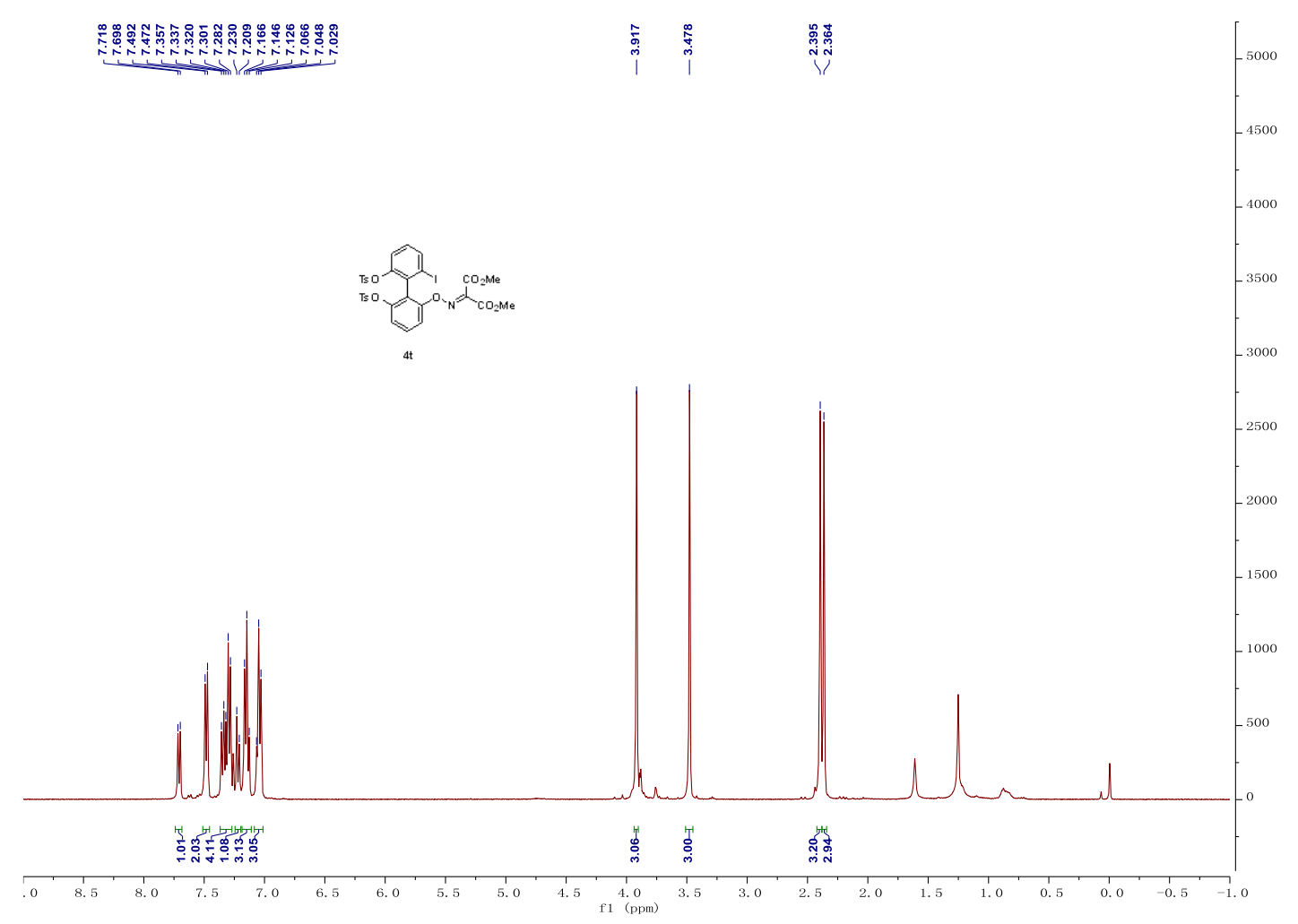

Figure $\mathbf{S 4 2} .{ }^{1} \mathrm{H}$ NMR spectra $\left(400 \mathrm{MHz}, \mathrm{CDCl}_{3}\right.$ ) of $\mathbf{4 t}$ 


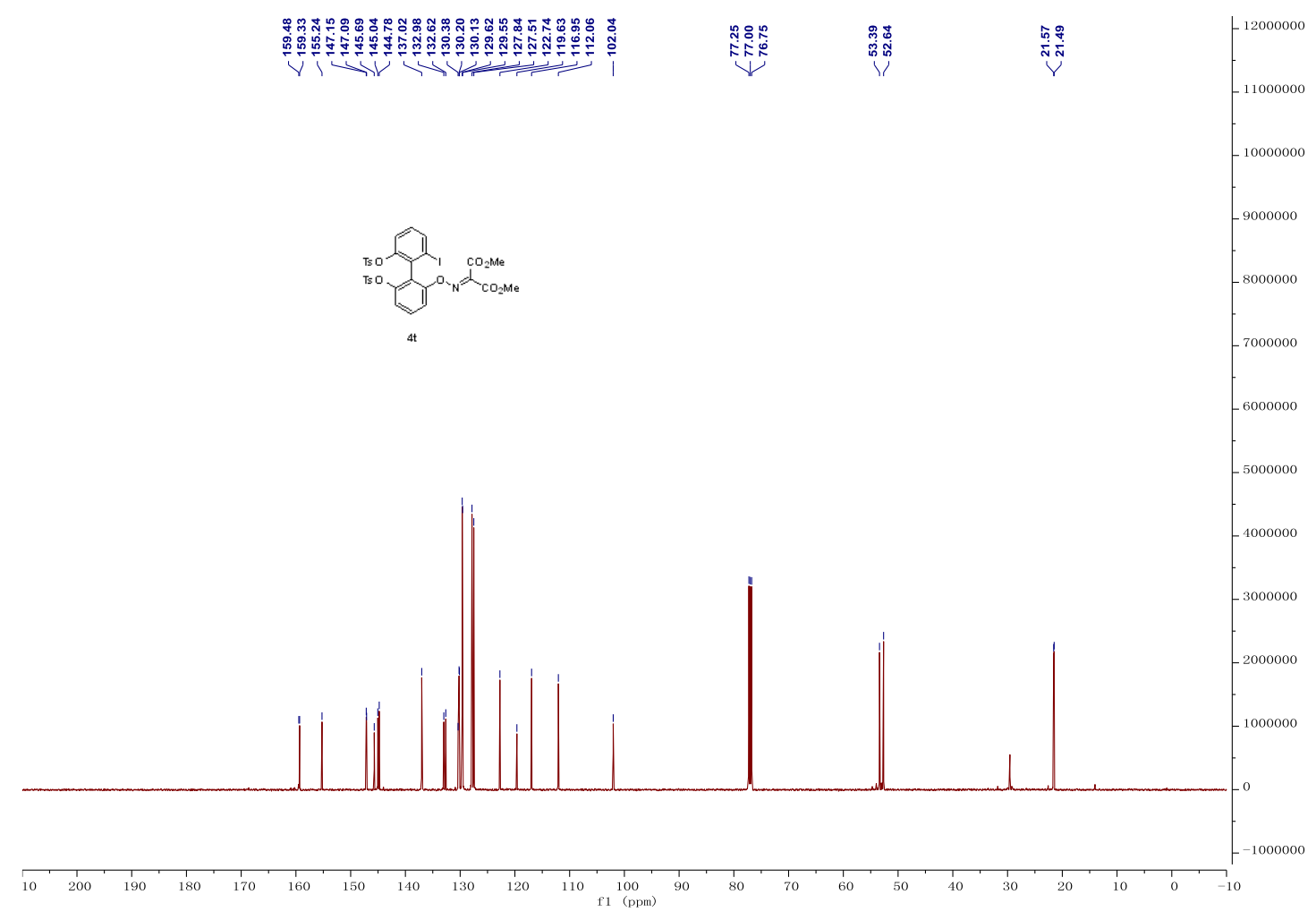

Figure $\mathbf{S 4 3} .{ }^{13} \mathrm{C}$ NMR spectra $\left(126 \mathrm{MHz}, \mathrm{CDCl}_{3}\right)$ of $\mathbf{4 t}$

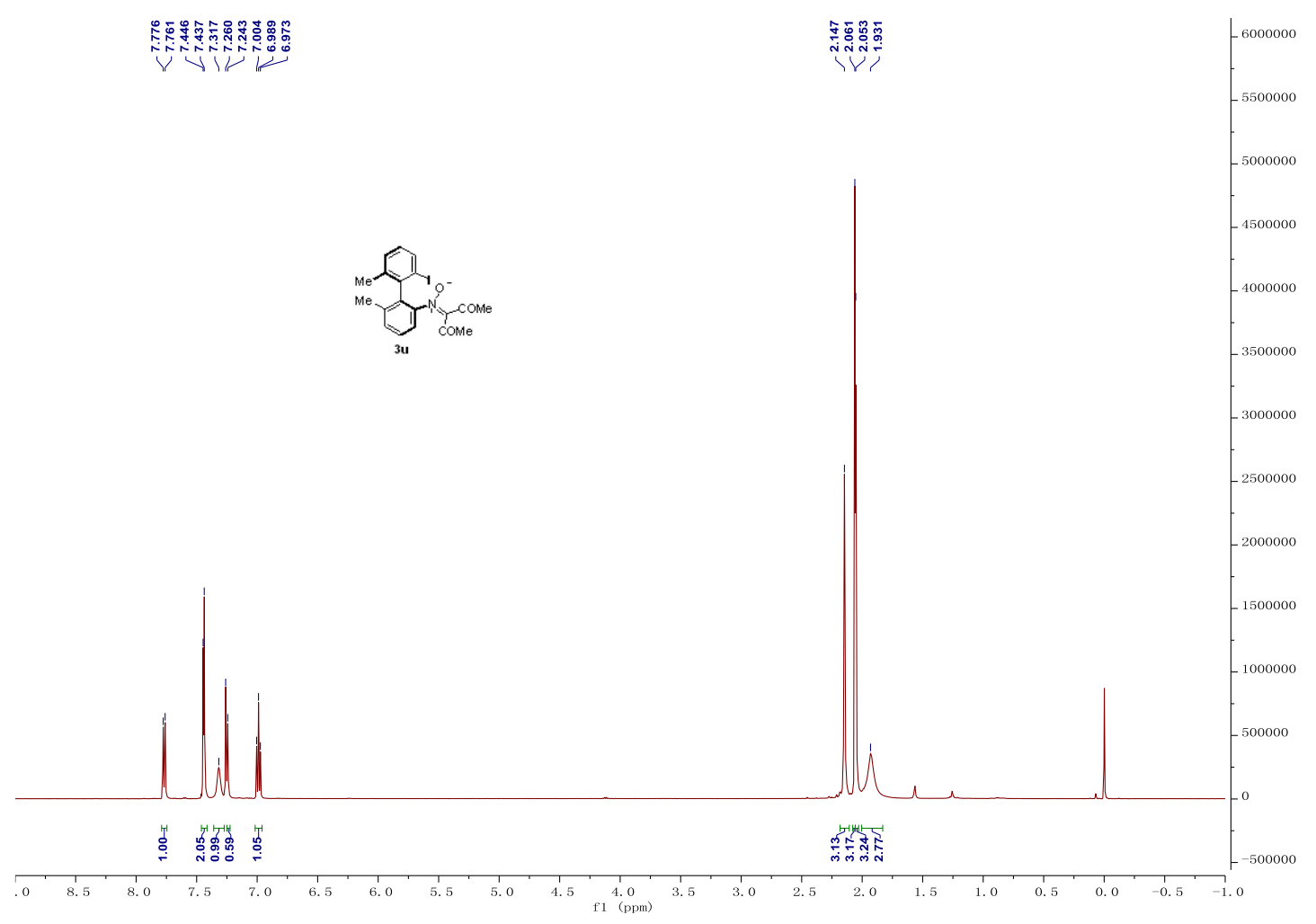

Figure S44. ${ }^{1} \mathrm{H}$ NMR spectra $\left(500 \mathrm{MHz}, \mathrm{CDCl}_{3}\right)$ of $\mathbf{3 u}$ 


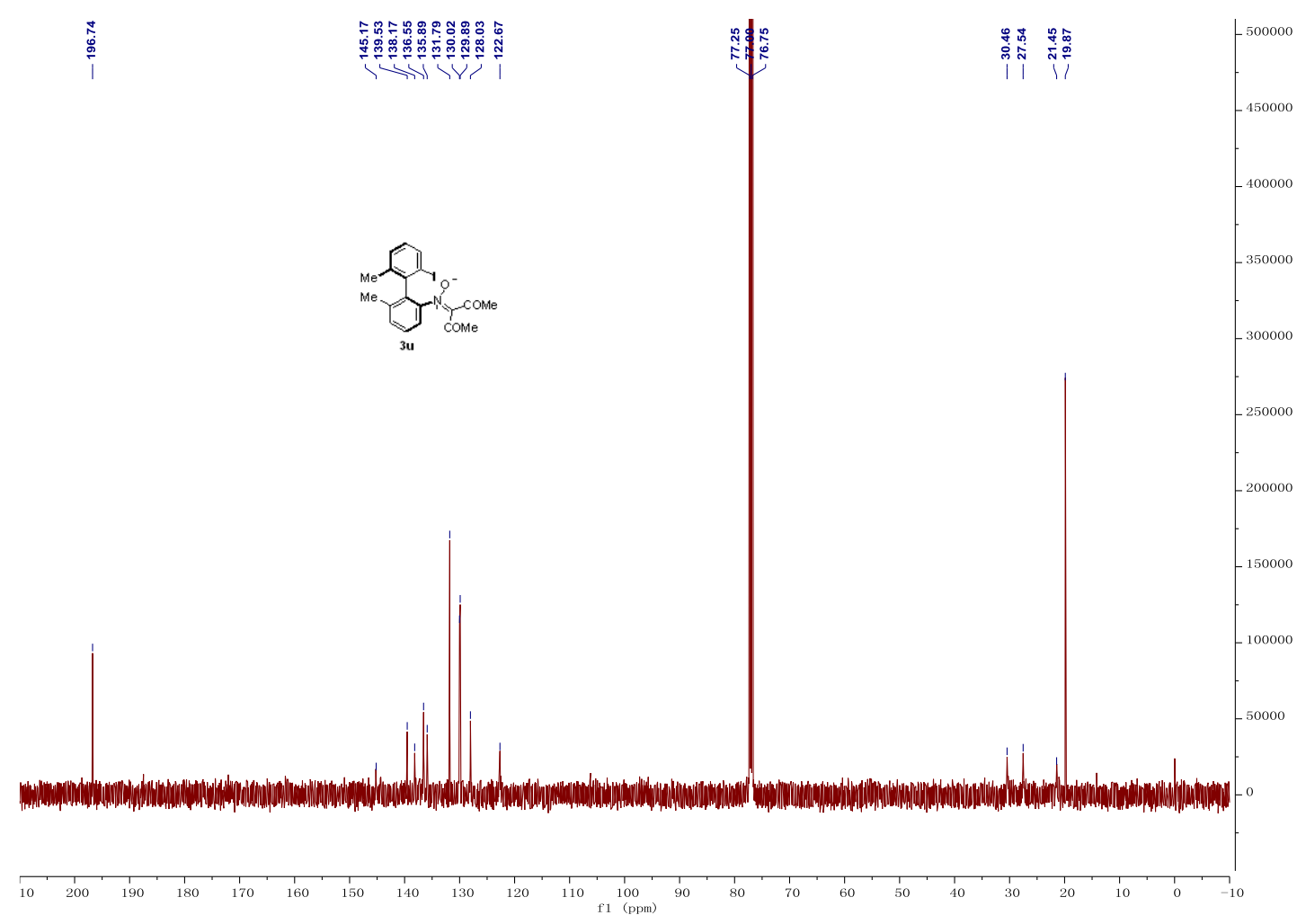

Figure $\mathbf{S 4 5} .{ }^{13} \mathrm{C}$ NMR spectra $\left(126 \mathrm{MHz}, \mathrm{CDCl}_{3}\right)$ of $\mathbf{3 u}$

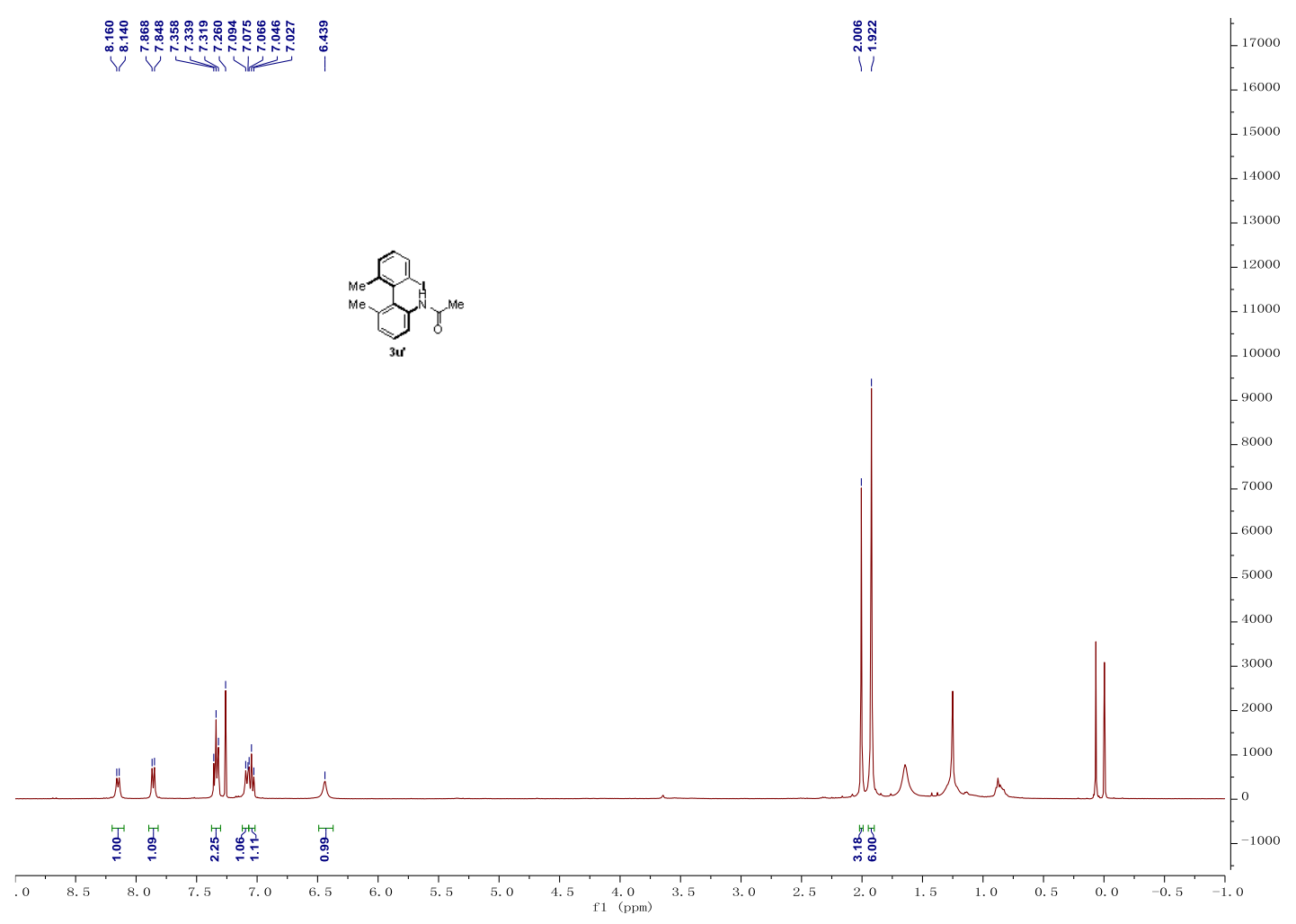

Figure S46. ${ }^{1} \mathrm{H}$ NMR spectra (400 $\mathrm{MHz}, \mathrm{CDCl}_{3}$ ) of 3u' 


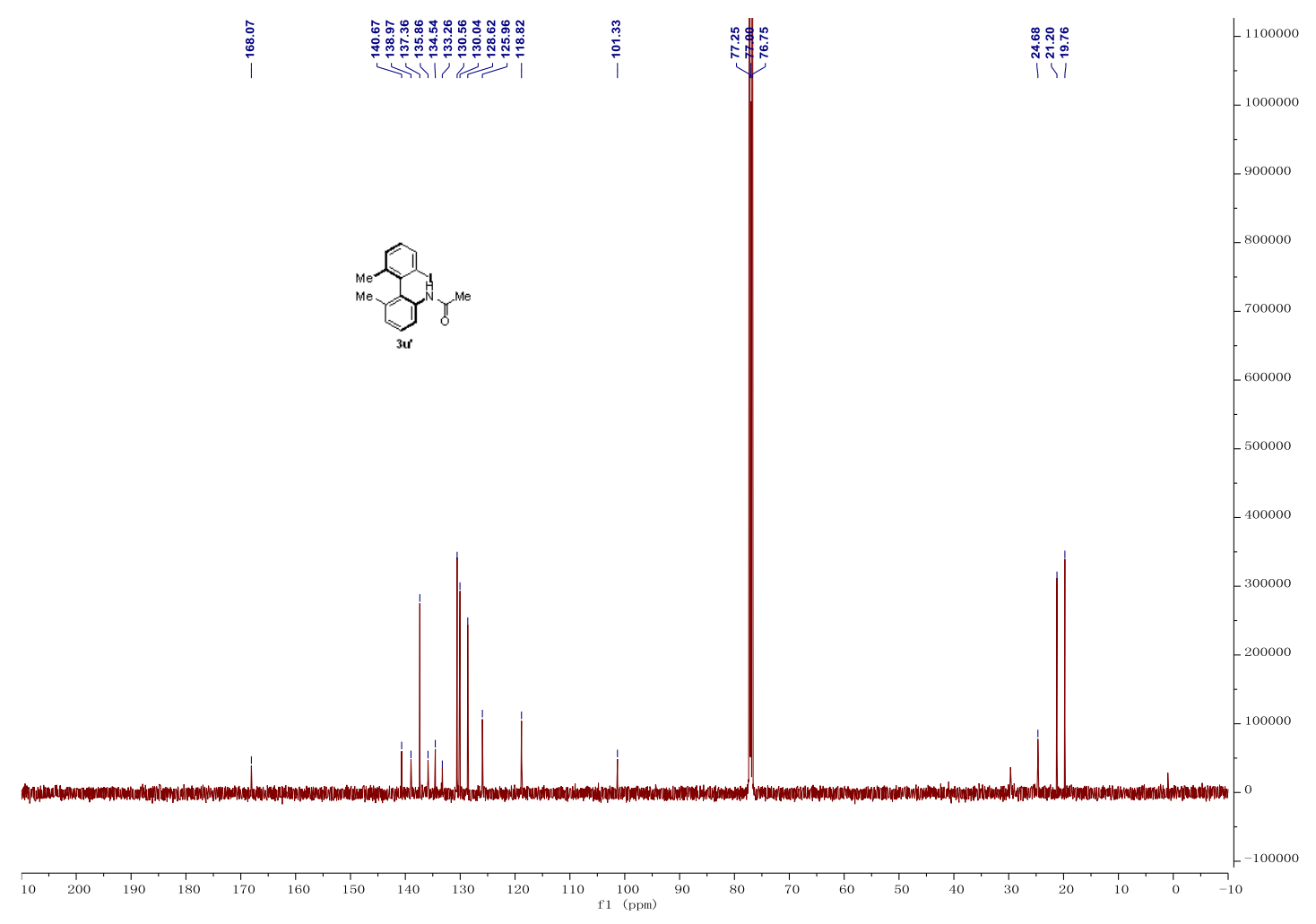

Figure S47. ${ }^{13} \mathrm{C}$ NMR spectra $\left(126 \mathrm{MHz}, \mathrm{CDCl}_{3}\right)$ of $\mathbf{3 u}$ '

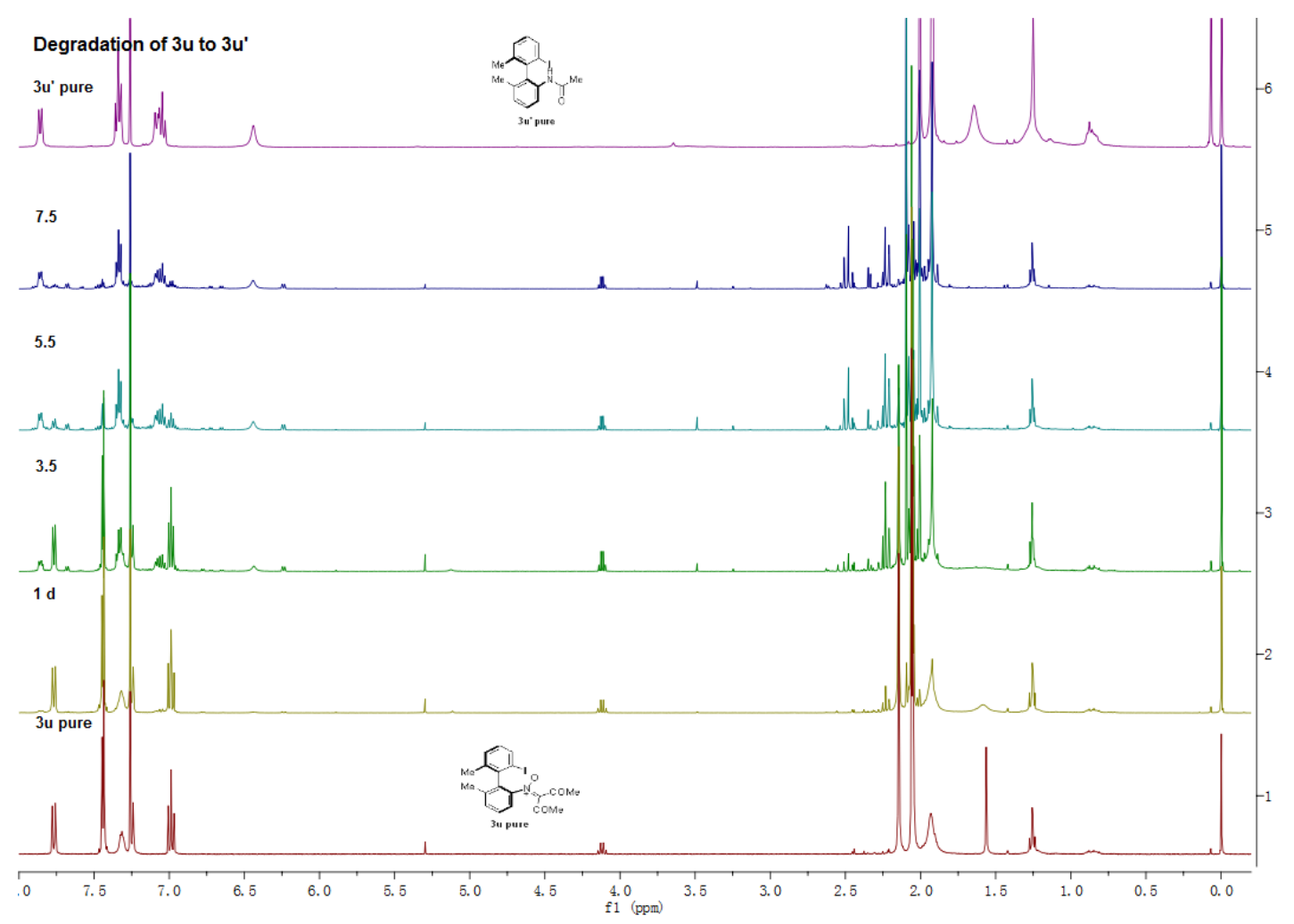

Figure S48. ${ }^{1} \mathrm{H}$ NMR spectra (400 or $500 \mathrm{MHz}, \mathrm{CDCl}_{3}$ ) of degradation of $\mathbf{3 u}$ to $\mathbf{3 u}$ ' 


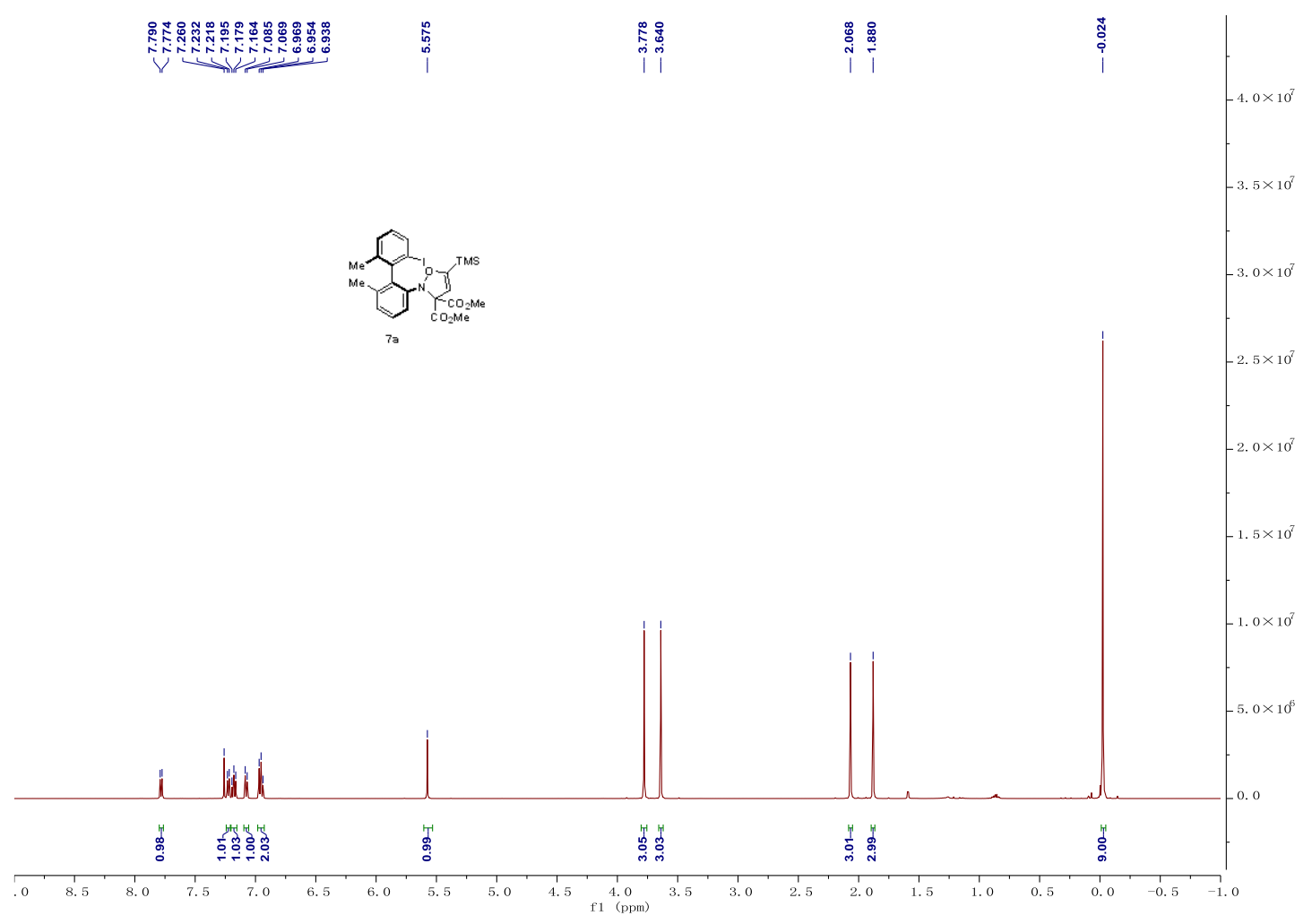

Figure S49. ${ }^{1} \mathrm{H}$ NMR spectra $\left(500 \mathrm{MHz}, \mathrm{CDCl}_{3}\right)$ of $\mathbf{7 a}$

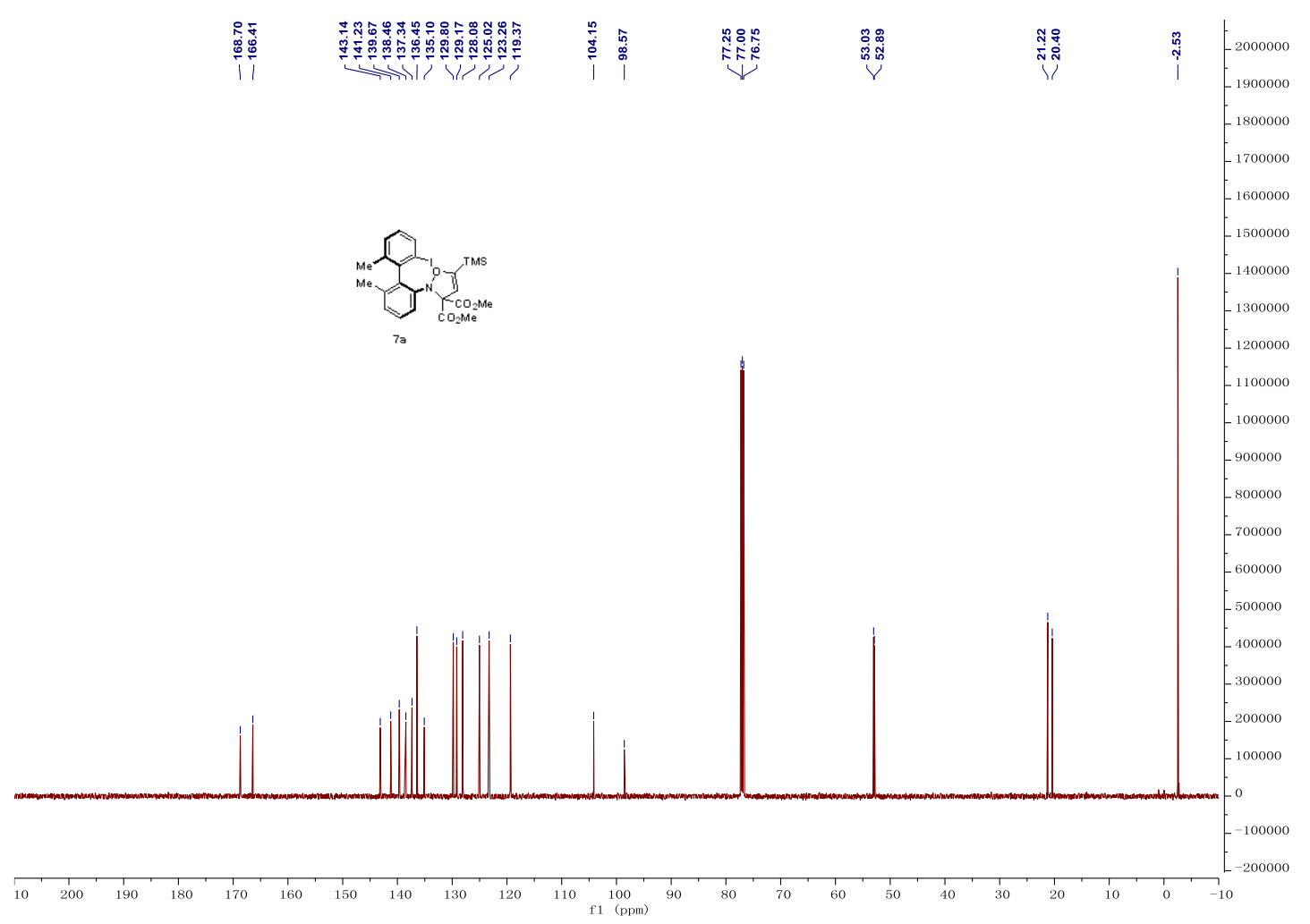

Figure S50. ${ }^{13} \mathrm{C}$ NMR spectra $\left(126 \mathrm{MHz}, \mathrm{CDCl}_{3}\right)$ of $\mathbf{7 a}$ 


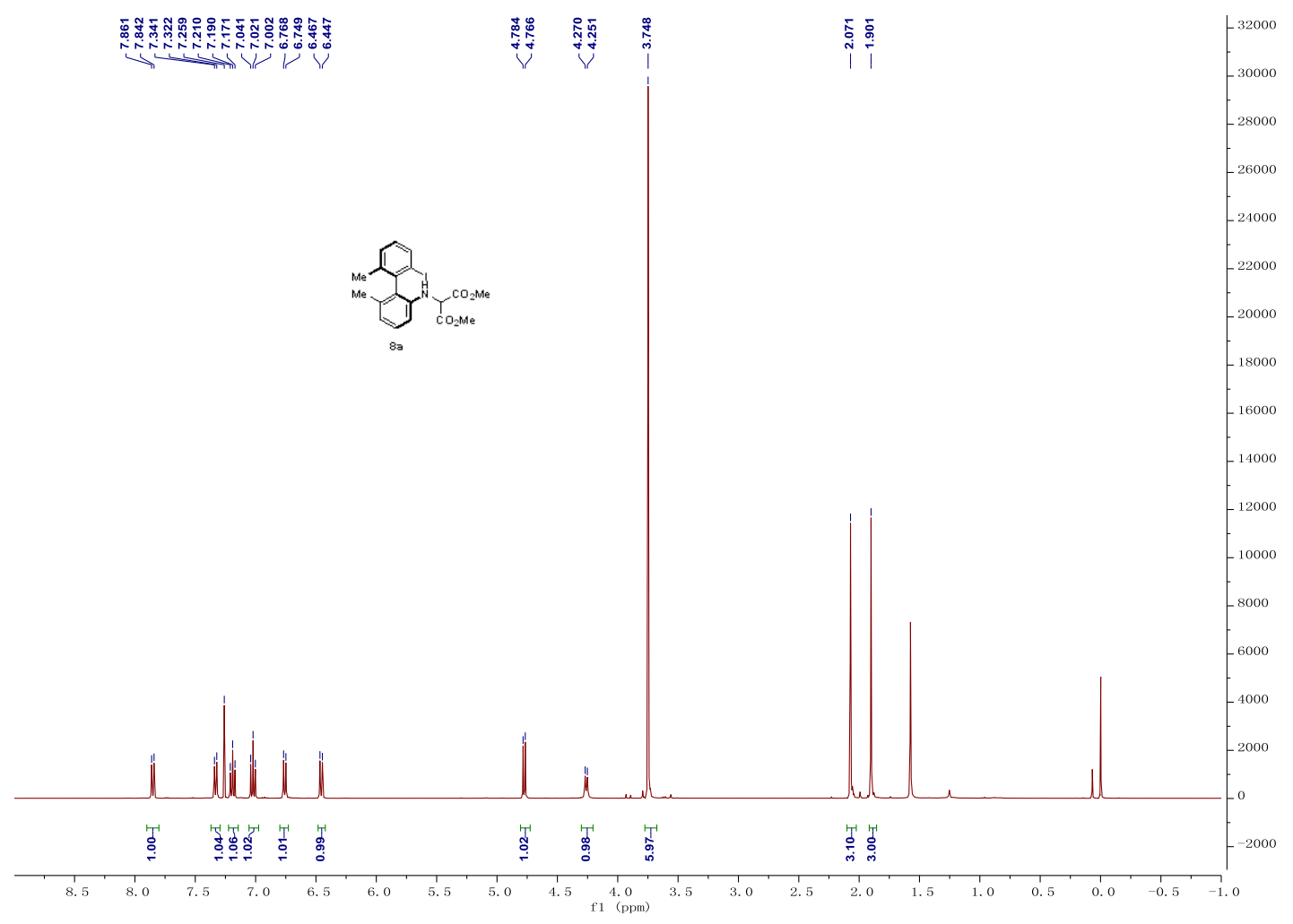

Figure S51. ${ }^{1} \mathrm{H}$ NMR spectra $\left(400 \mathrm{MHz}, \mathrm{CDCl}_{3}\right)$ of $\mathbf{8 a}$

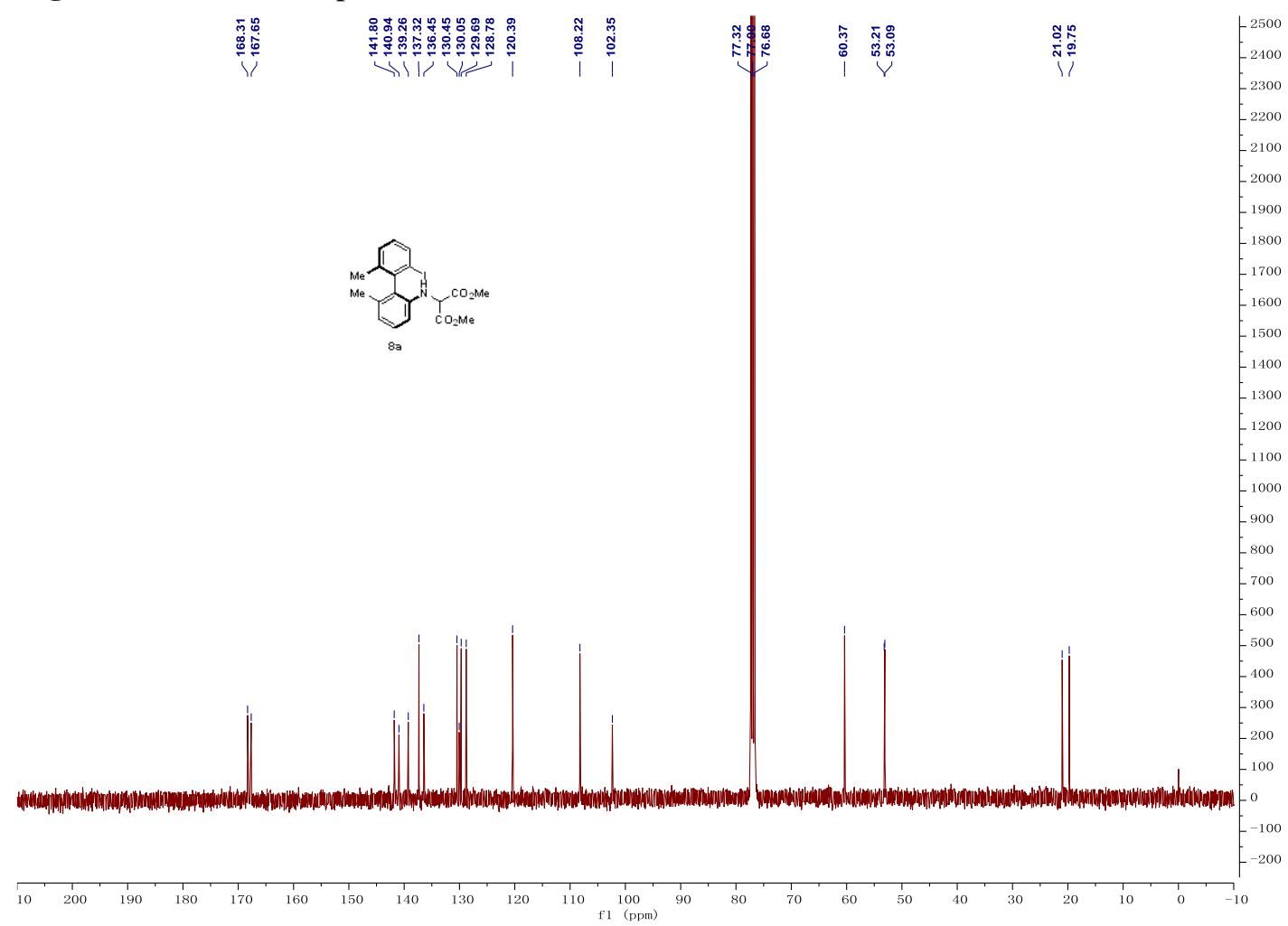

Figure S52. ${ }^{13} \mathrm{C}$ NMR spectra $\left(101 \mathrm{MHz}, \mathrm{CDCl}_{3}\right)$ of $\mathbf{8 a}$ 


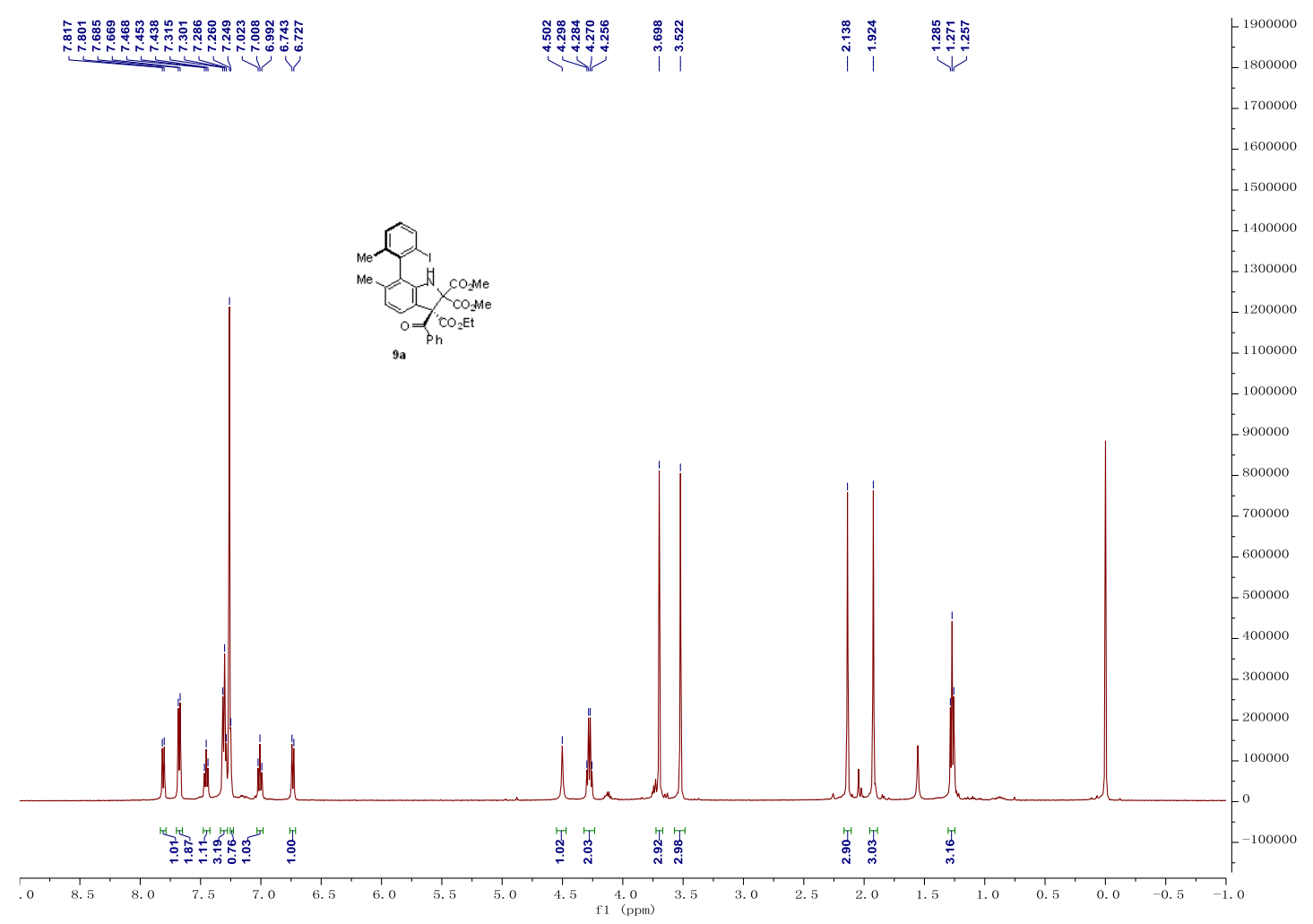

Figure S53. ${ }^{1} \mathrm{H}$ NMR spectra $\left(500 \mathrm{MHz}, \mathrm{CDCl}_{3}\right)$ of $\mathbf{9 a}$

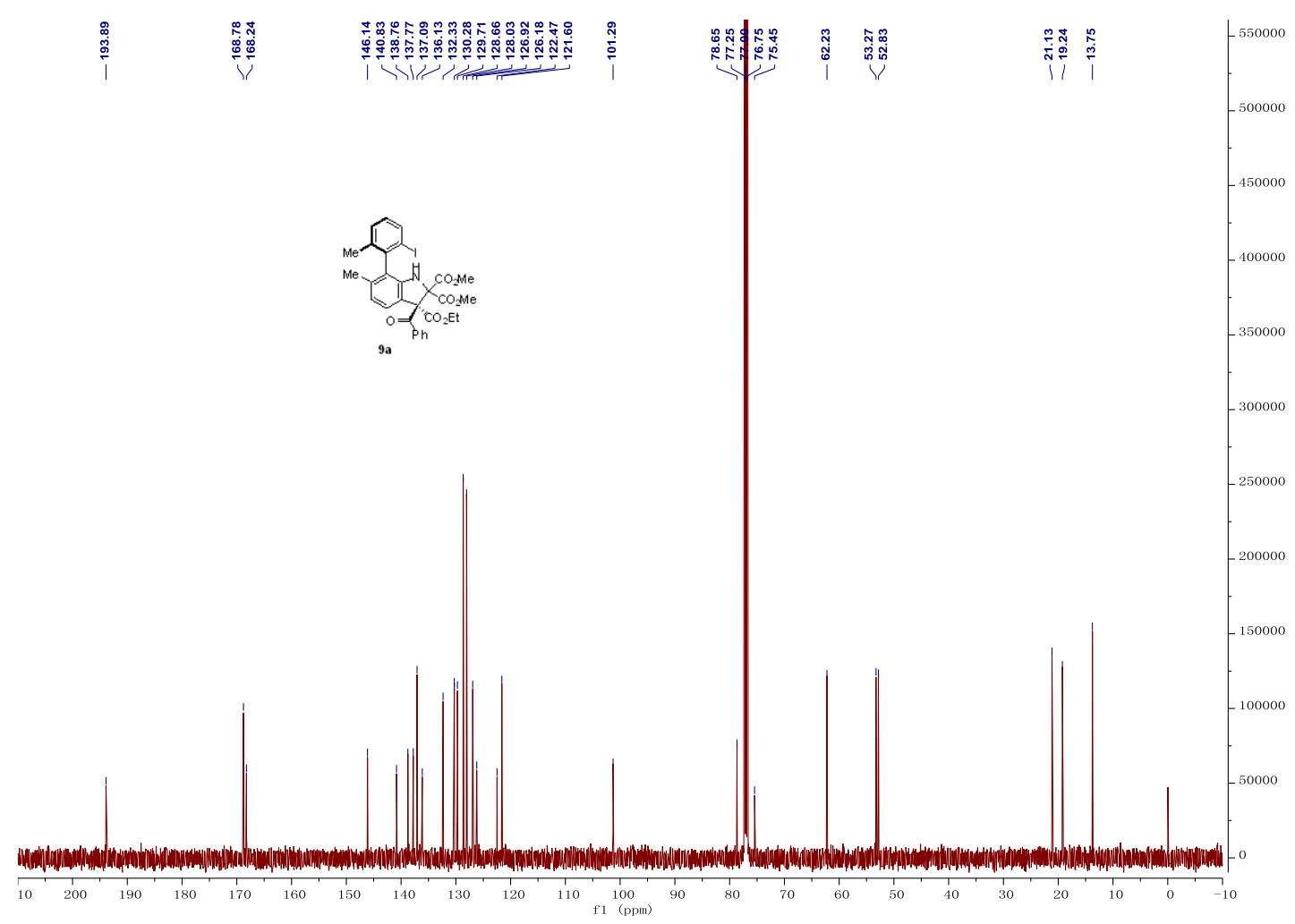

Figure S54. ${ }^{13} \mathrm{C}$ NMR spectra $\left(126 \mathrm{MHz}, \mathrm{CDCl}_{3}\right)$ of $9 \mathbf{a}$ 


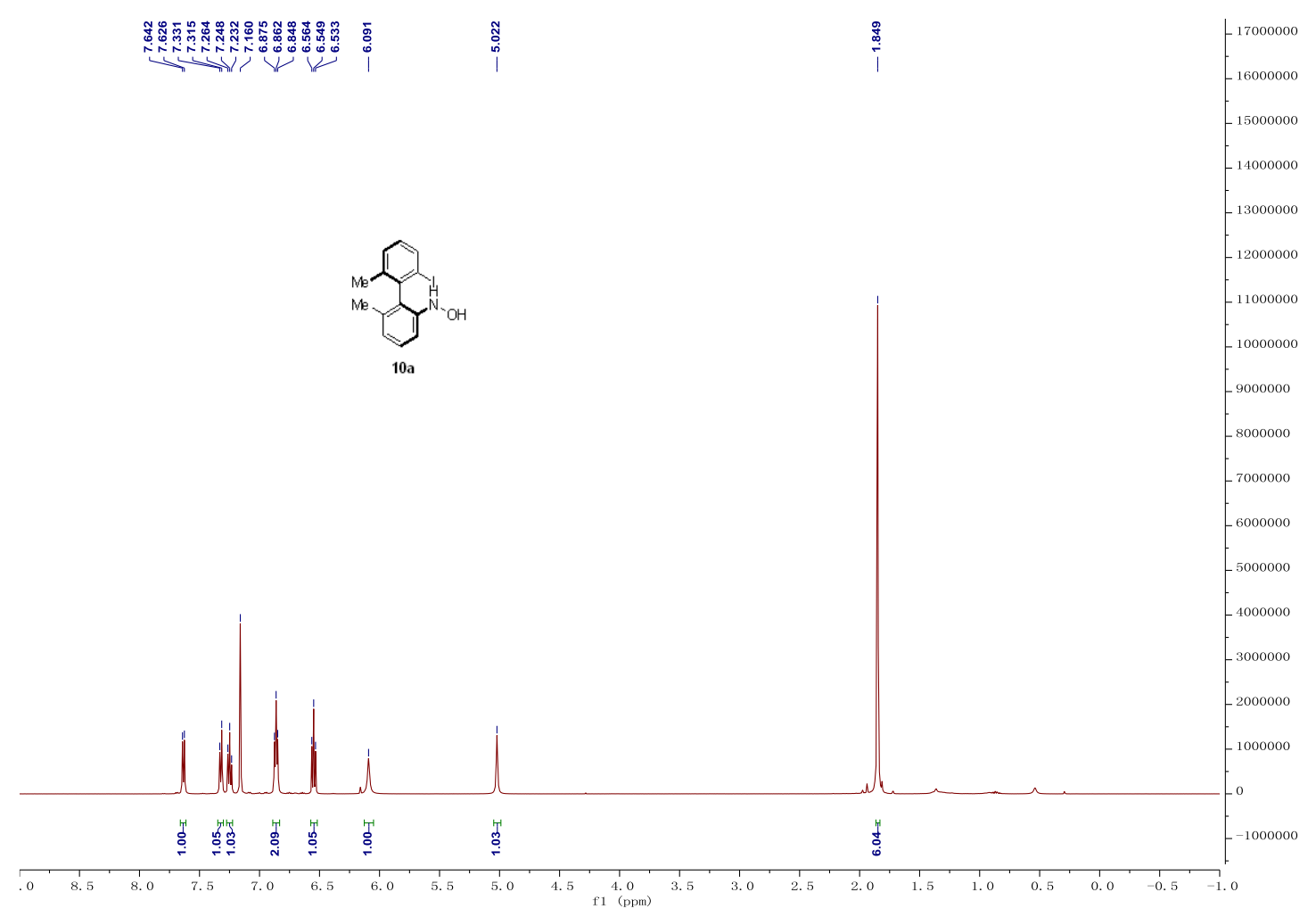

Figure S55. ${ }^{1} \mathrm{H}$ NMR spectra $\left(500 \mathrm{MHz}, \mathrm{C}_{6} \mathrm{D}_{6}\right)$ of 10a

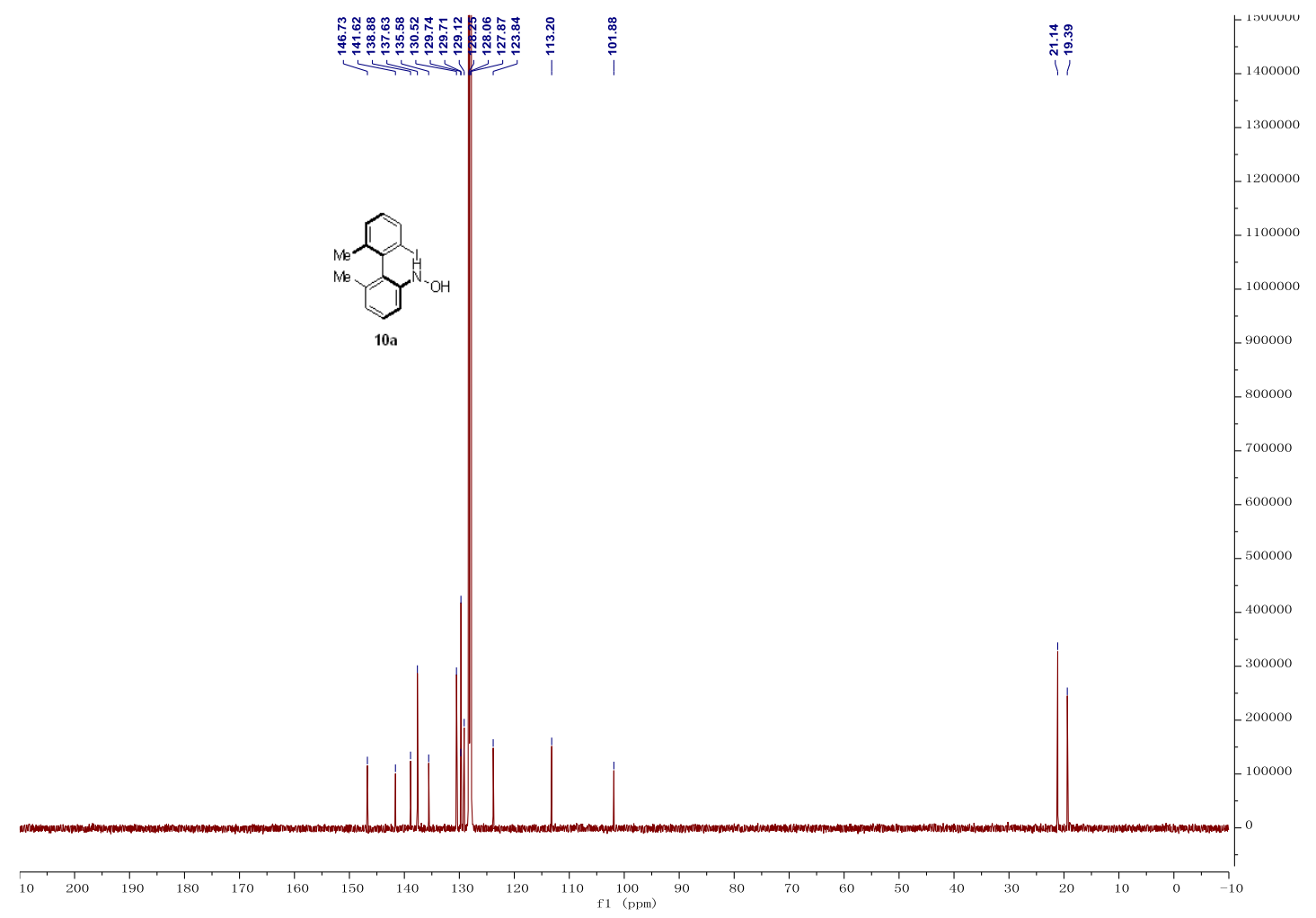

Figure S56. ${ }^{13} \mathrm{C}$ NMR spectra $\left(126 \mathrm{MHz}, \mathrm{C}_{6} \mathrm{D}_{6}\right)$ of $\mathbf{1 0 a}$ 


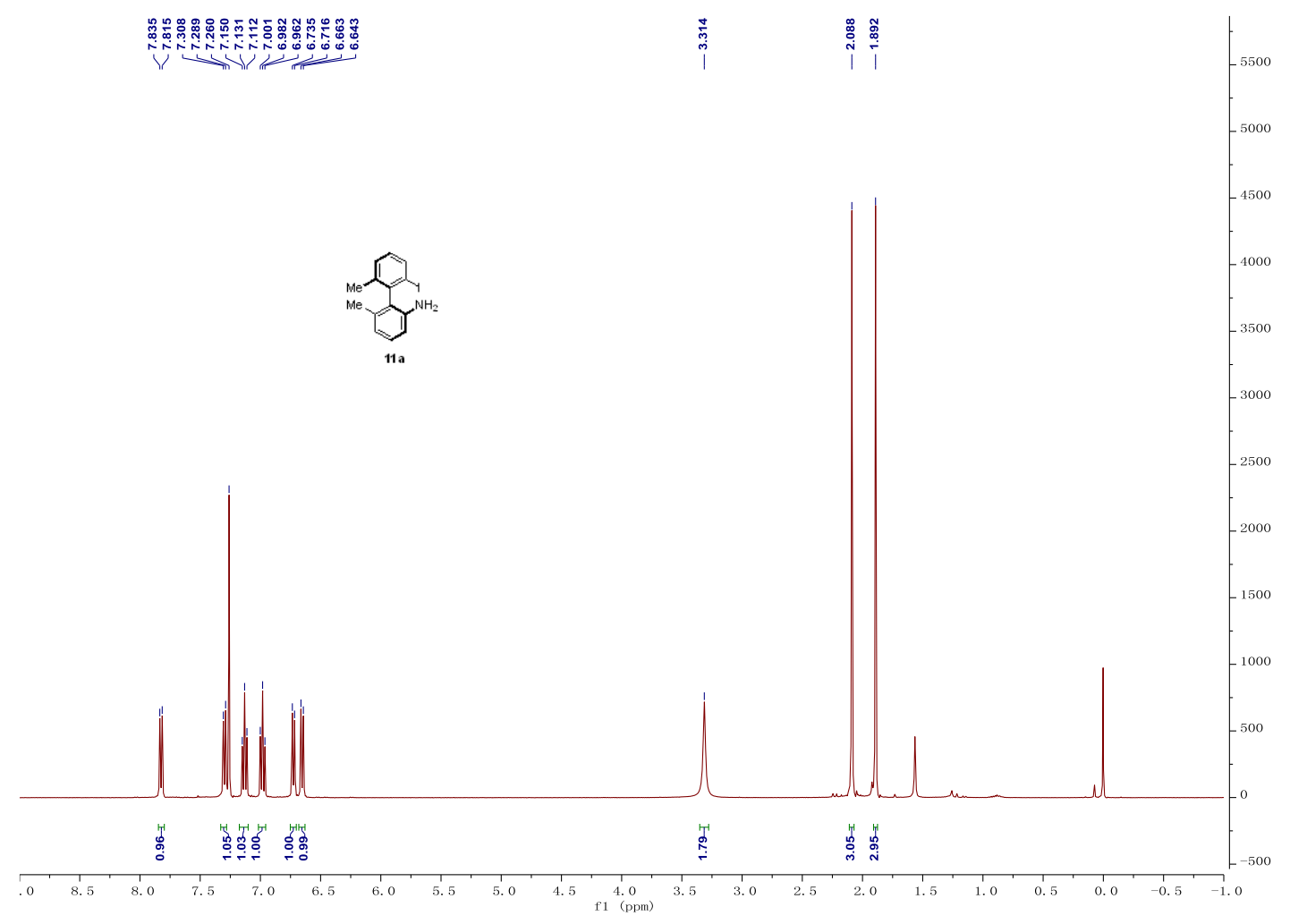

Figure S57. ${ }^{1} \mathrm{H}$ NMR spectra $\left(400 \mathrm{MHz}, \mathrm{CDCl}_{3}\right)$ of $\mathbf{1 1 a}$

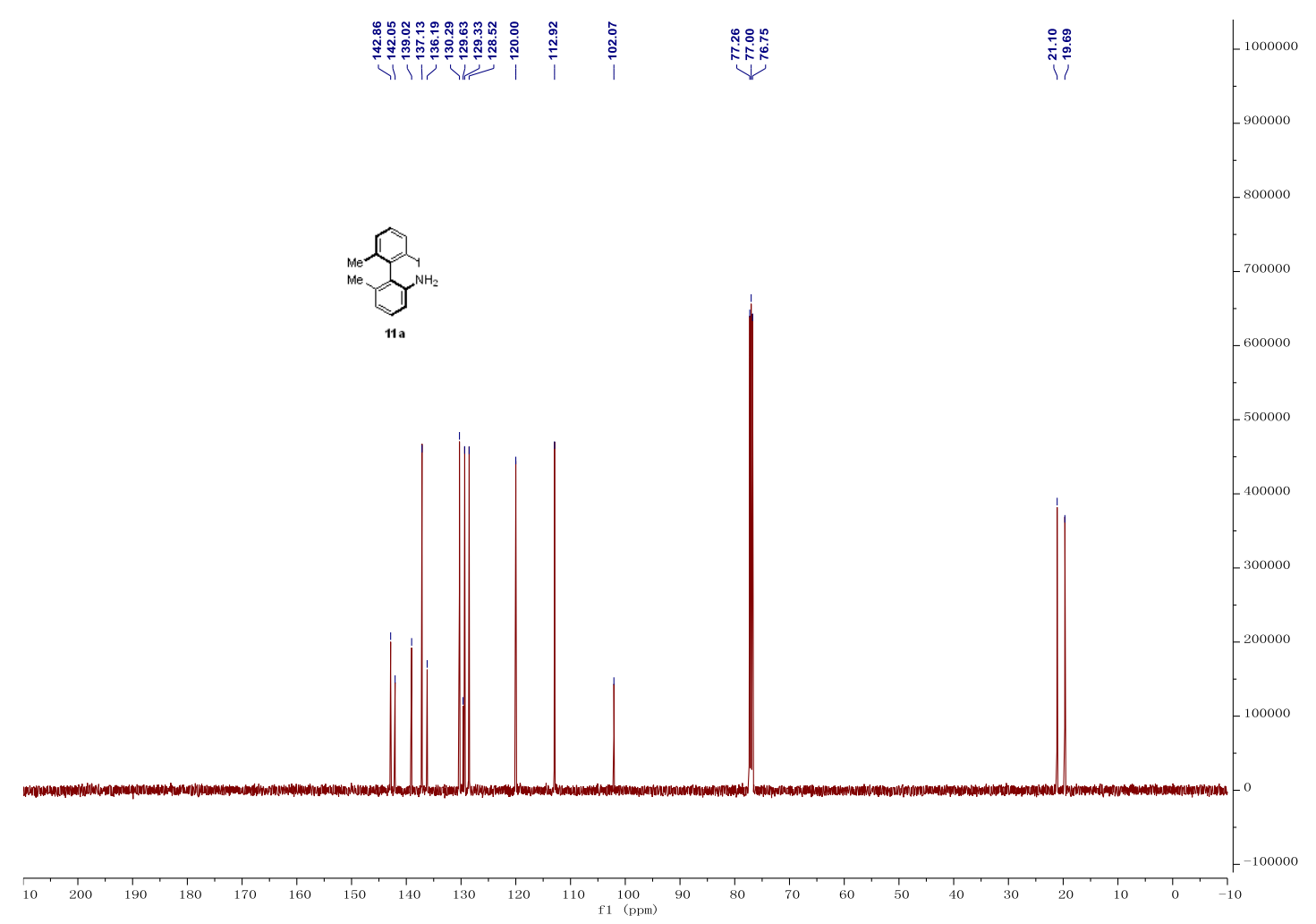

Figure S58. ${ }^{13} \mathrm{C}$ NMR spectra $\left(126 \mathrm{MHz}, \mathrm{CDCl}_{3}\right)$ of 11a 


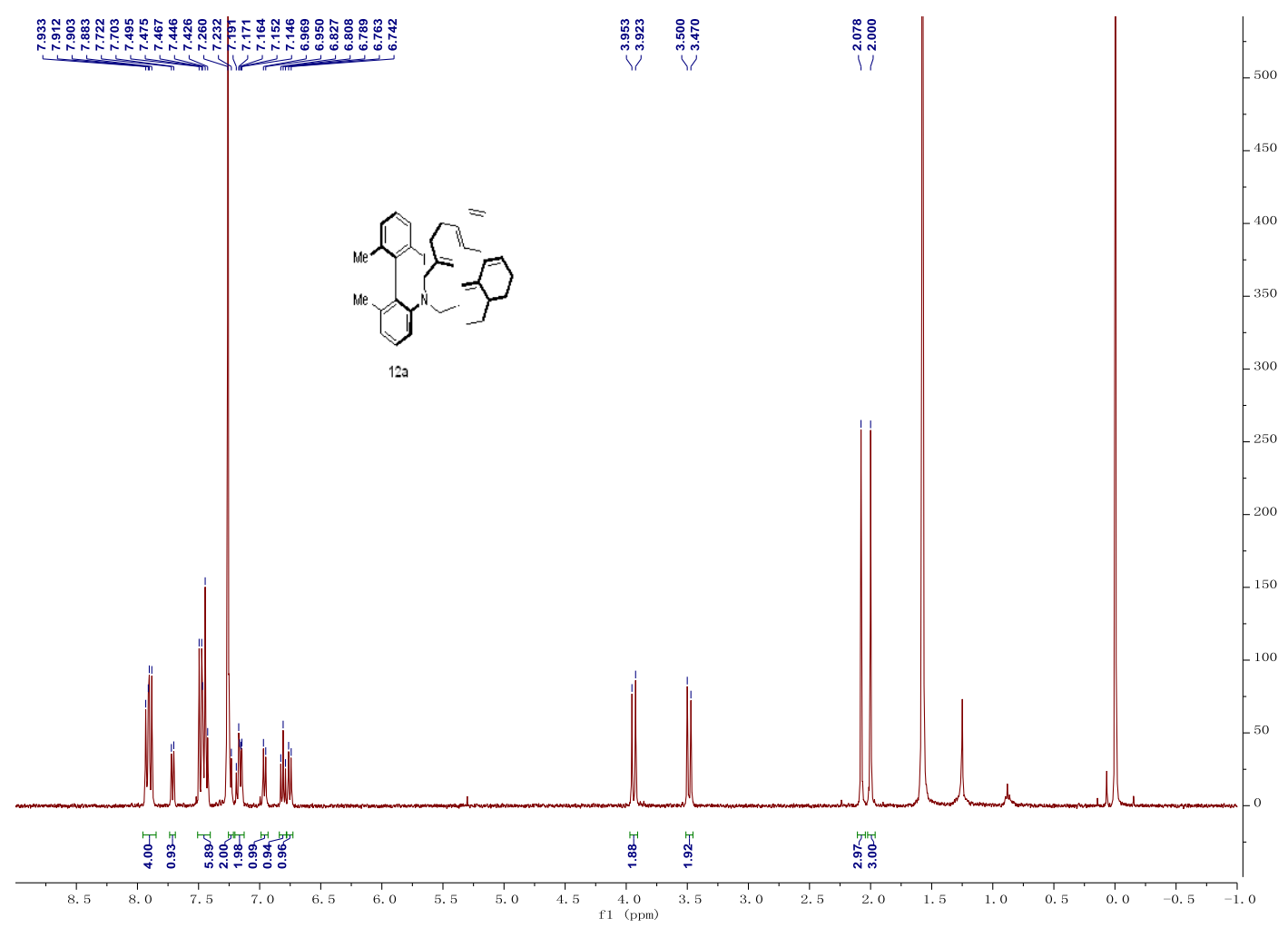

Figure S59. ${ }^{1} \mathrm{H}$ NMR spectra $\left(400 \mathrm{MHz}, \mathrm{CDCl}_{3}\right)$ of $\mathbf{1 2 a}$

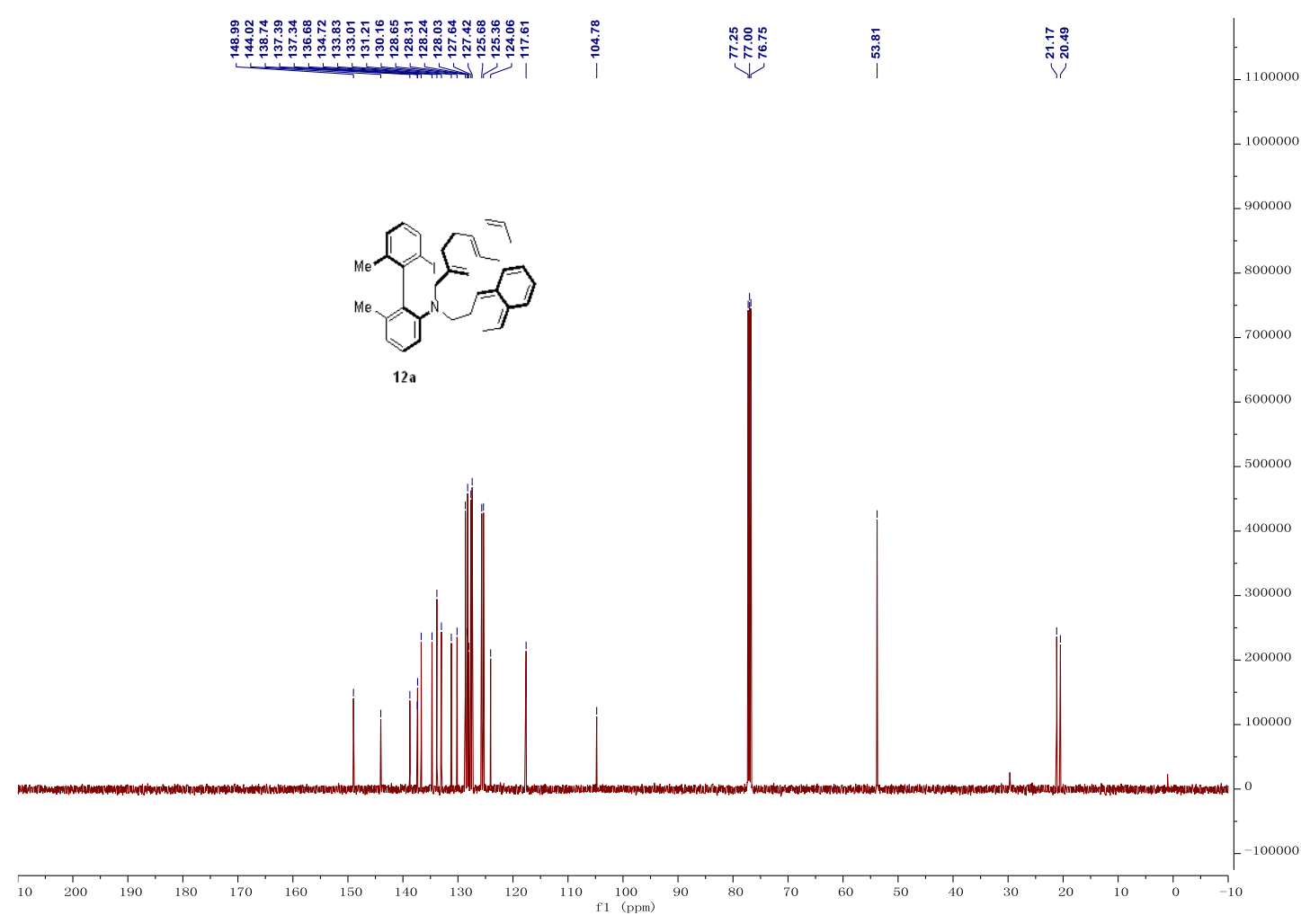

Figure S60. ${ }^{13} \mathrm{C}$ NMR spectra $\left(126 \mathrm{MHz}, \mathrm{CDCl}_{3}\right)$ of $\mathbf{1 2 a}$ 


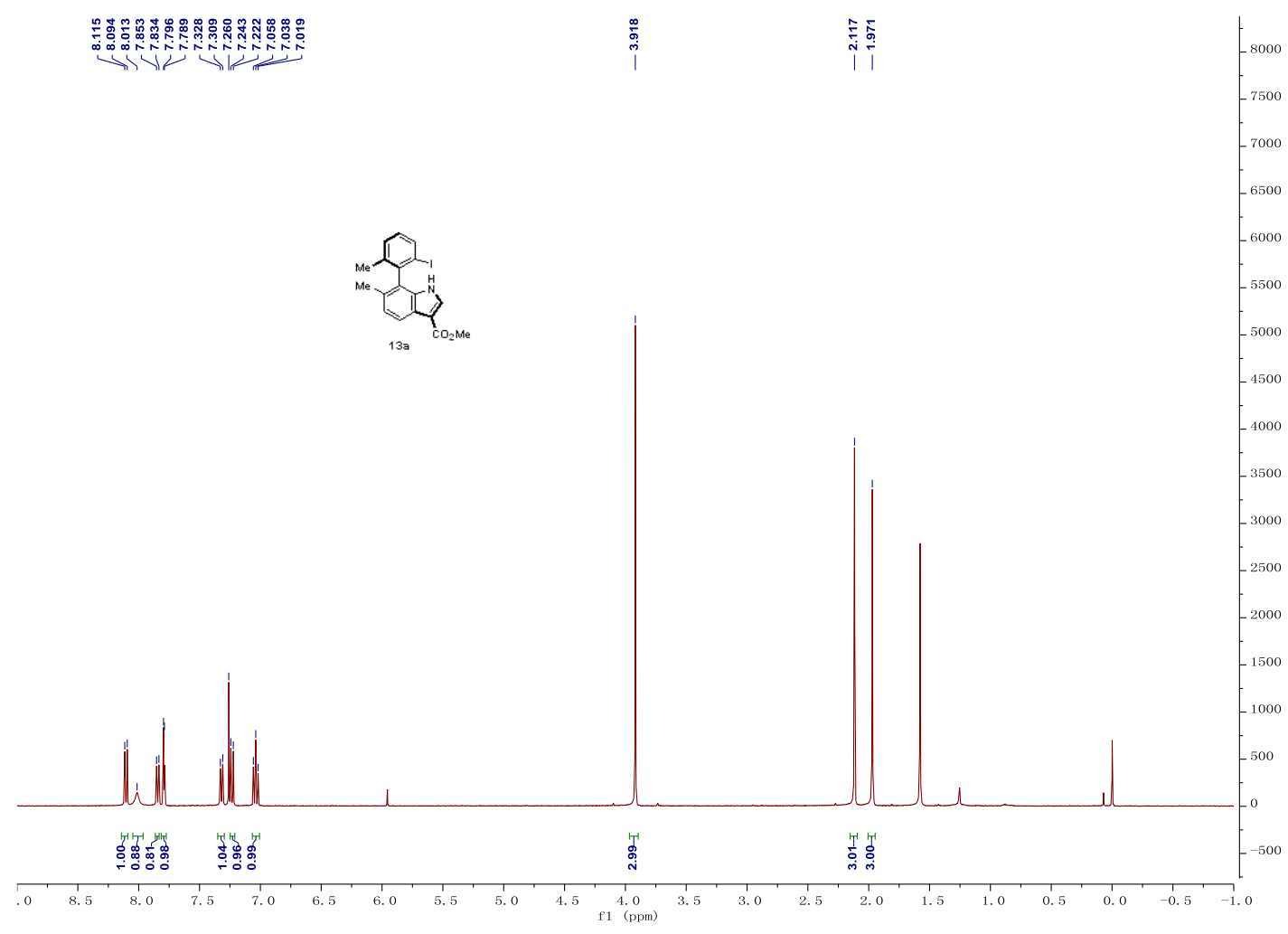

Figure S61. ${ }^{1} \mathrm{H}$ NMR spectra $\left(400 \mathrm{MHz}, \mathrm{CDCl}_{3}\right)$ of 13a

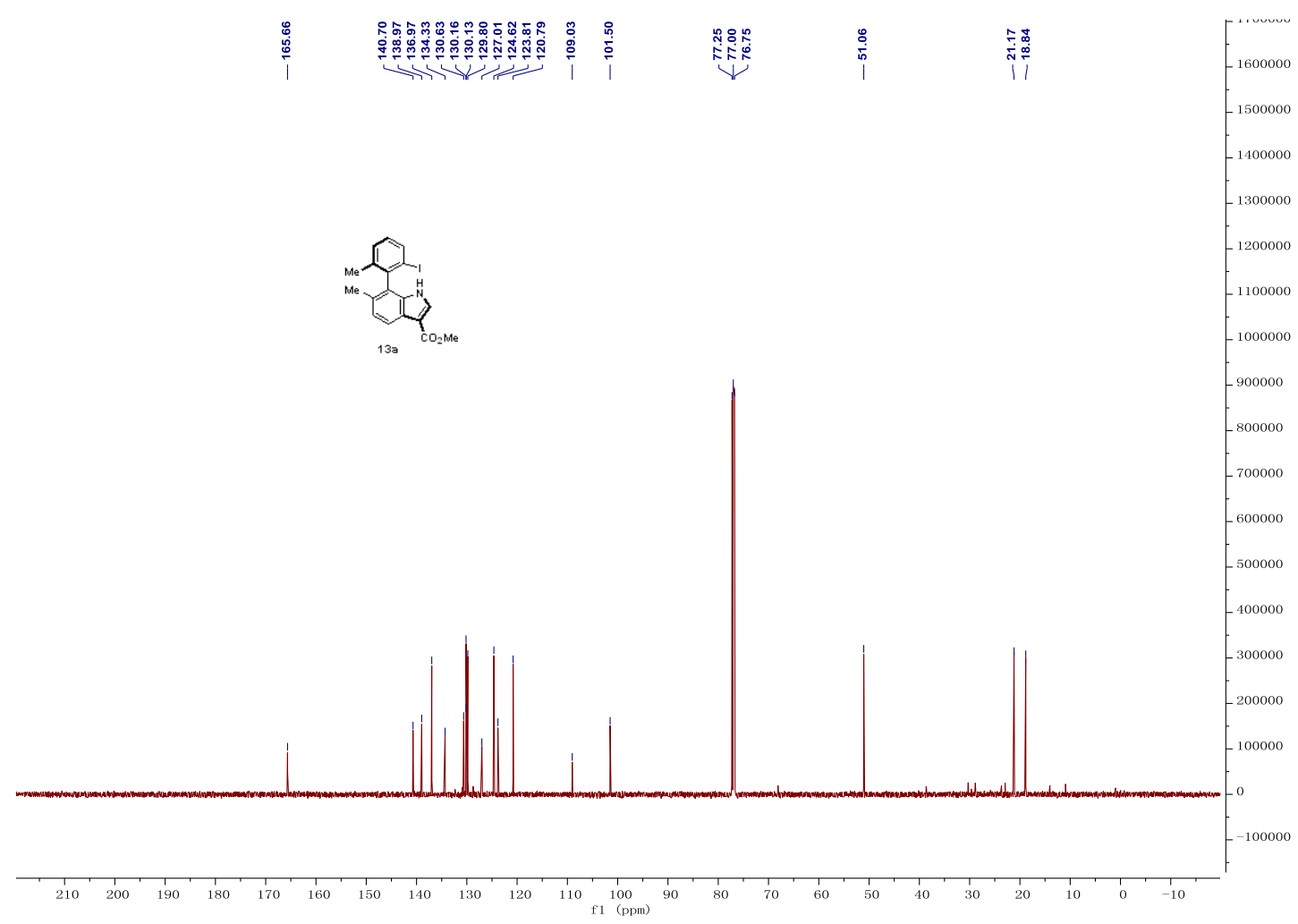

Figure S62. ${ }^{13} \mathrm{C}$ NMR spectra $\left(126 \mathrm{MHz}, \mathrm{CDCl}_{3}\right)$ of $\mathbf{1 3 a}$ 


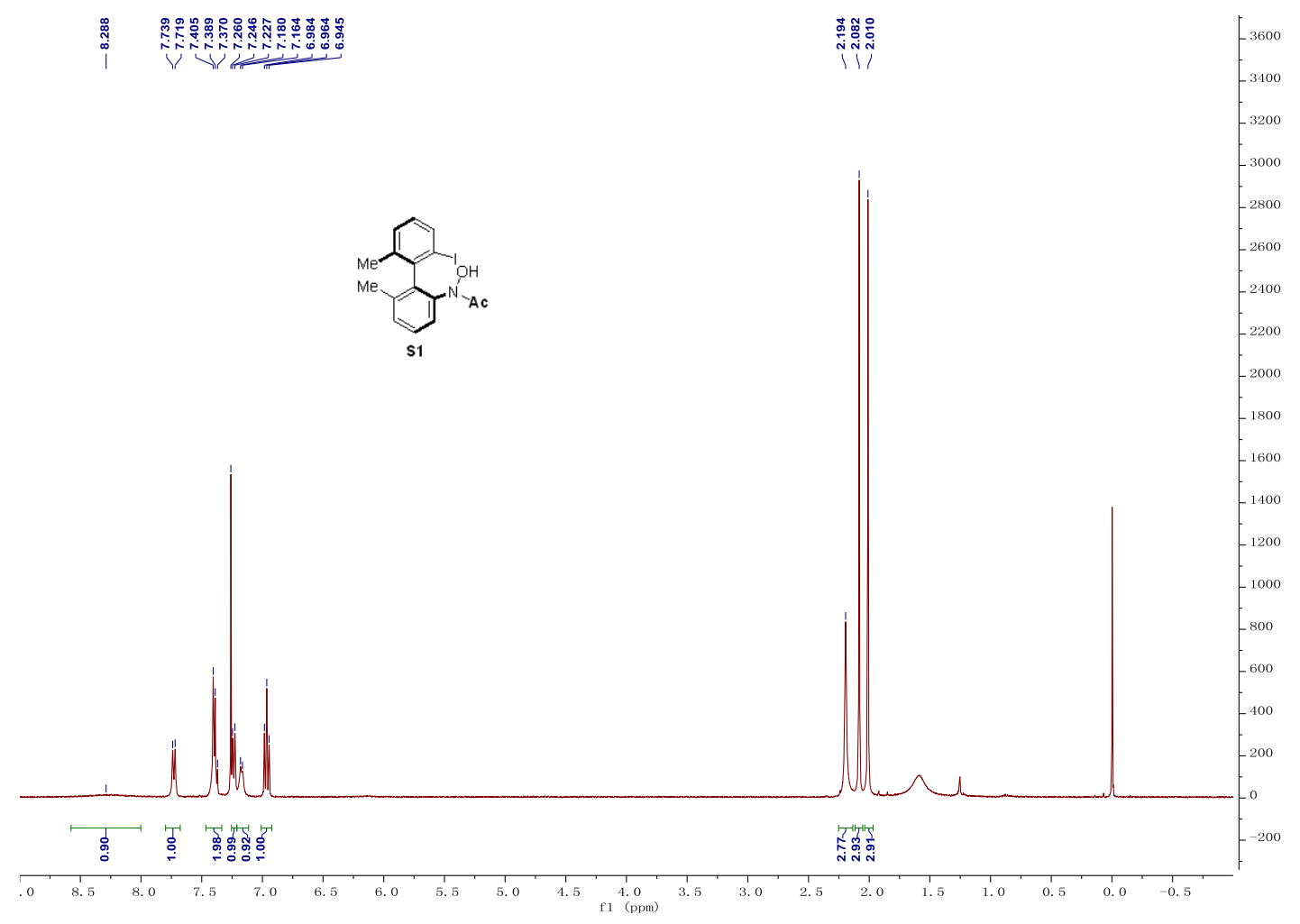

Figure S63. ${ }^{1} \mathrm{H}$ NMR spectra $\left(400 \mathrm{MHz}, \mathrm{CDCl}_{3}\right)$ of $\mathbf{S 1}$

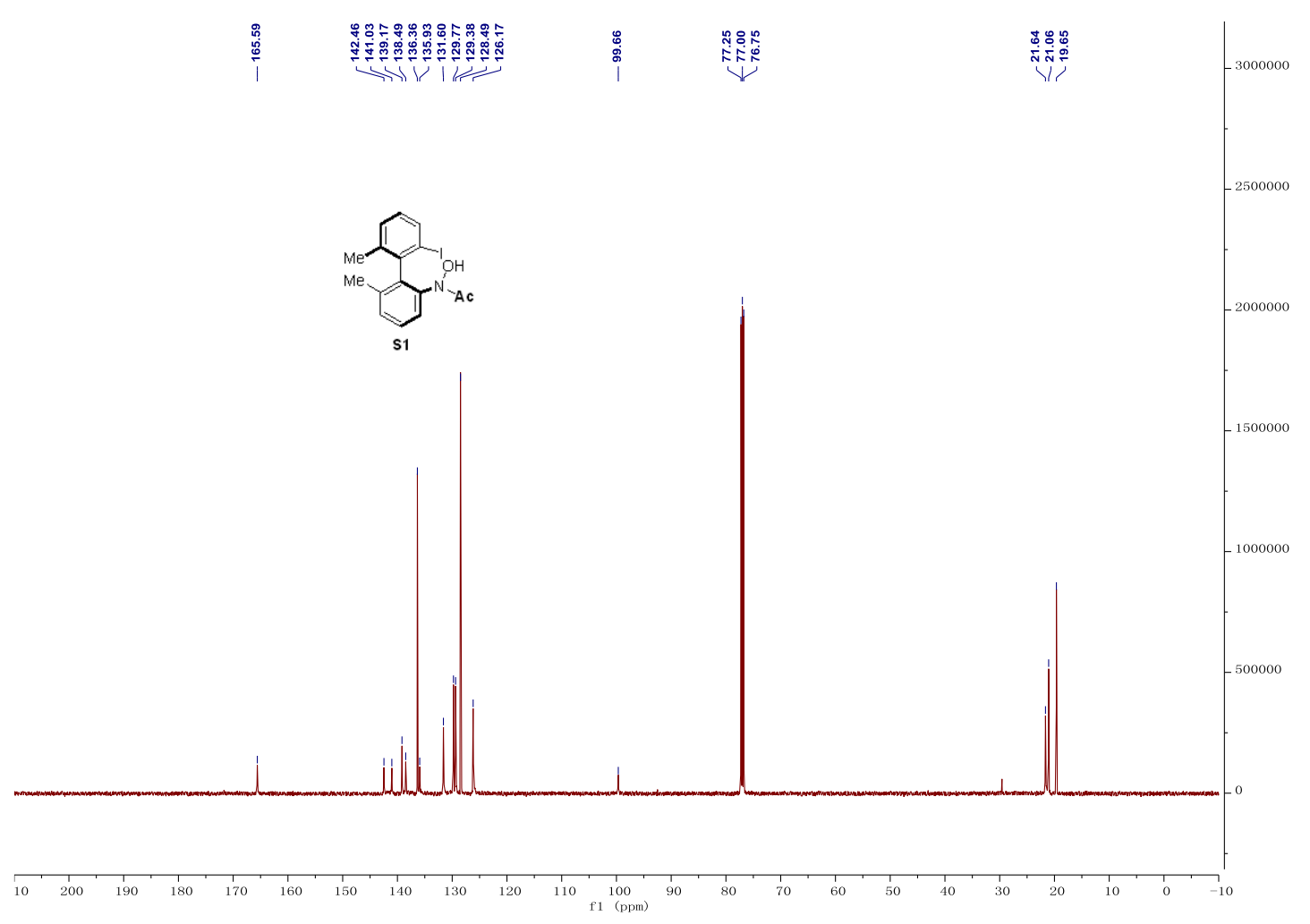

Figure S64. ${ }^{13} \mathrm{C}$ NMR spectra $\left(126 \mathrm{MHz}, \mathrm{CDCl}_{3}\right)$ of $\mathbf{S 1}$ 


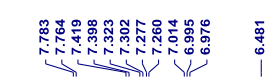

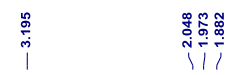
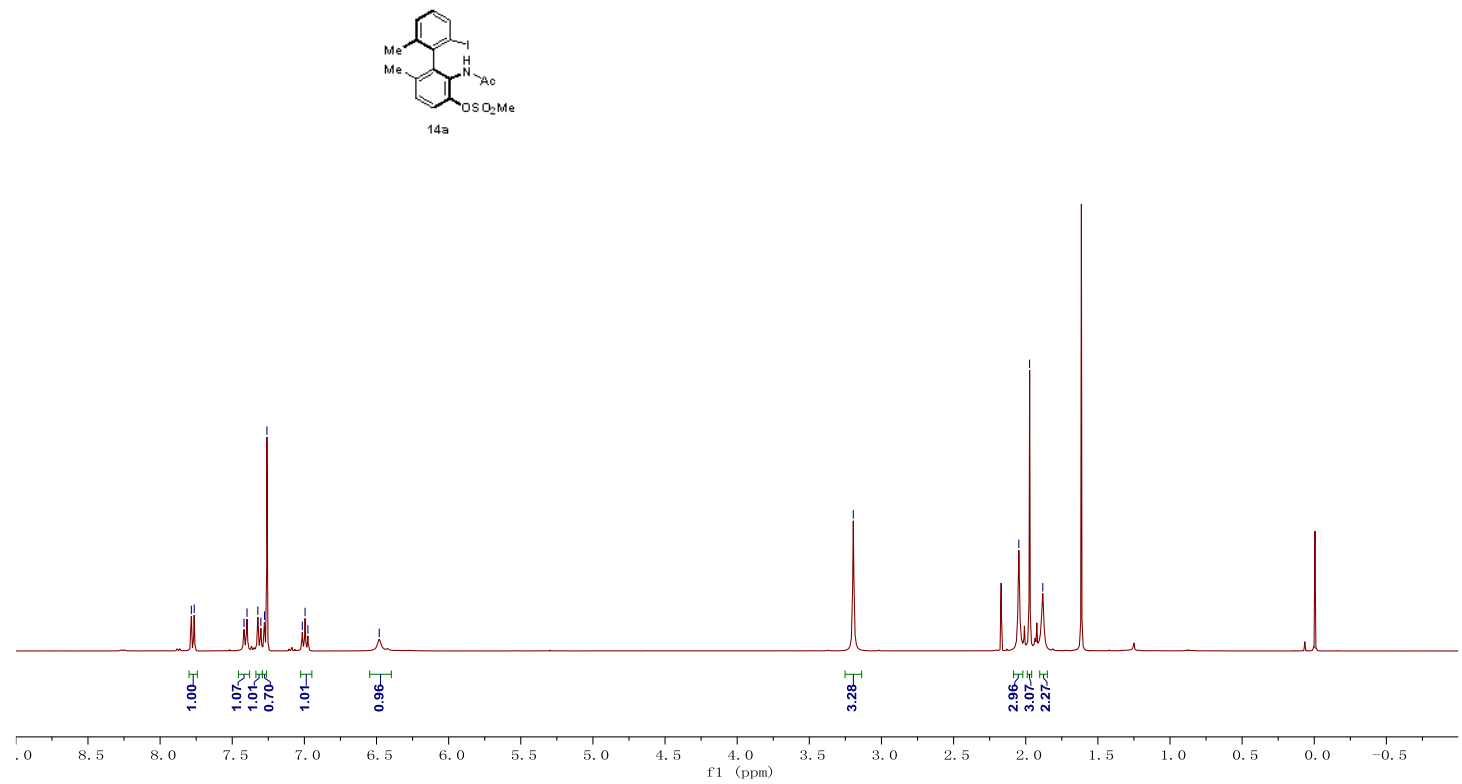

Figure $\mathbf{S 6 5} .{ }^{1} \mathrm{H}$ NMR spectra $\left(400 \mathrm{MHz}, \mathrm{CDCl}_{3}\right)$ of $\mathbf{1 4 a}$

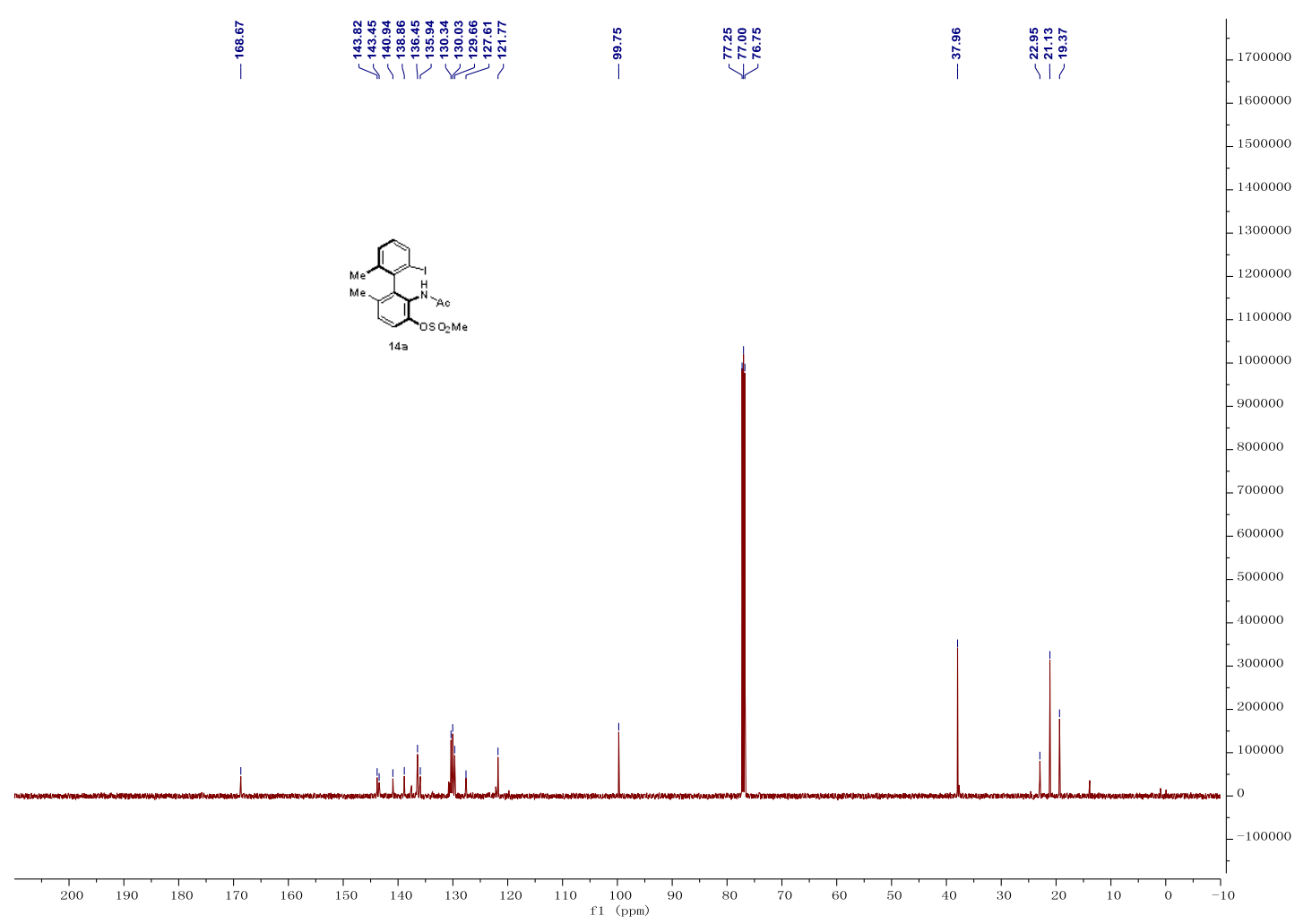

Figure S66. ${ }^{13} \mathrm{C}$ NMR spectra $\left(126 \mathrm{MHz}, \mathrm{CDCl}_{3}\right)$ of $\mathbf{1 4 a}$ 


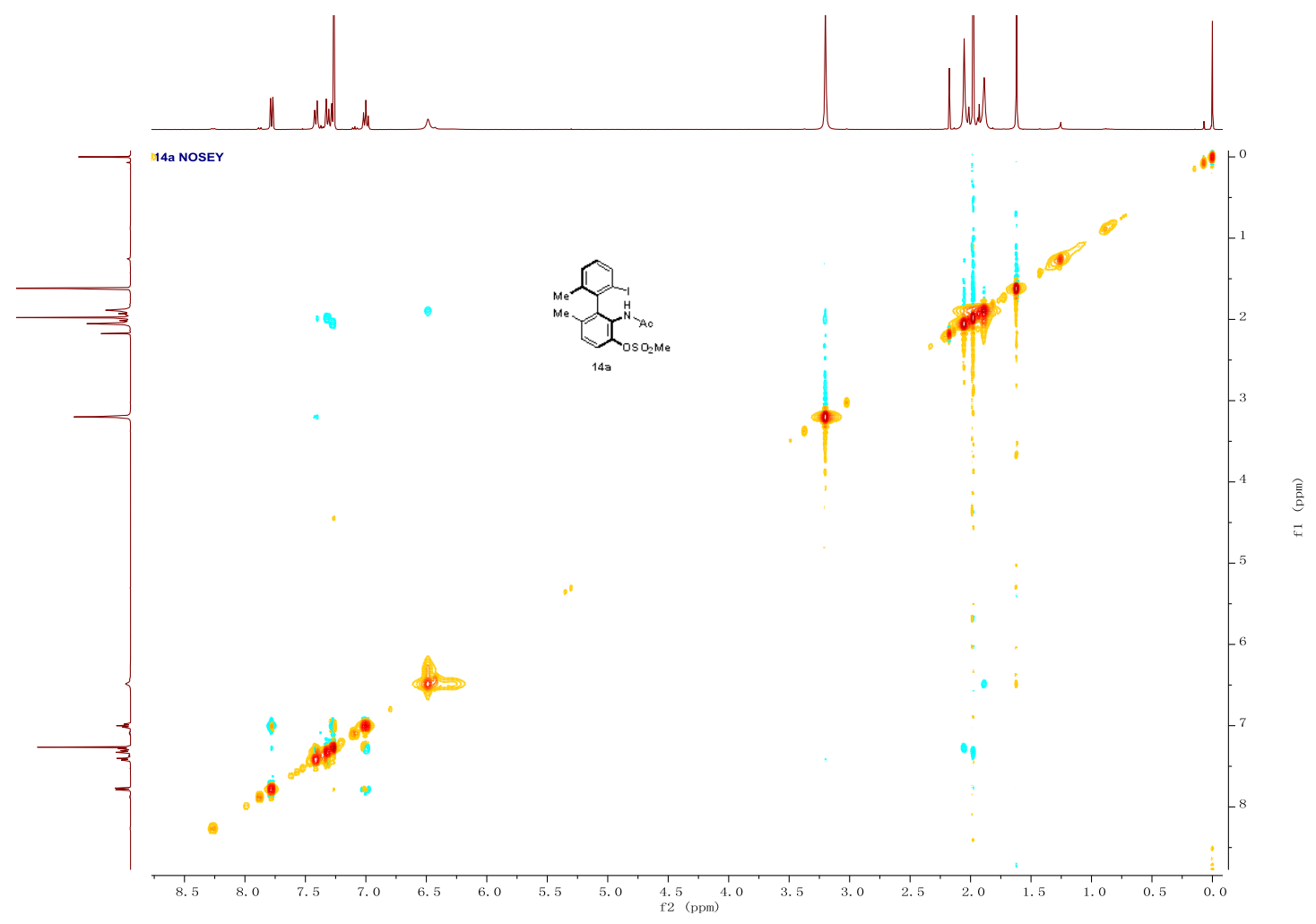

Figure S67. NOSEY NMR spectra (400 MHz, $\left.\mathrm{CDCl}_{3}\right)$ of $\mathbf{1 4 a}$

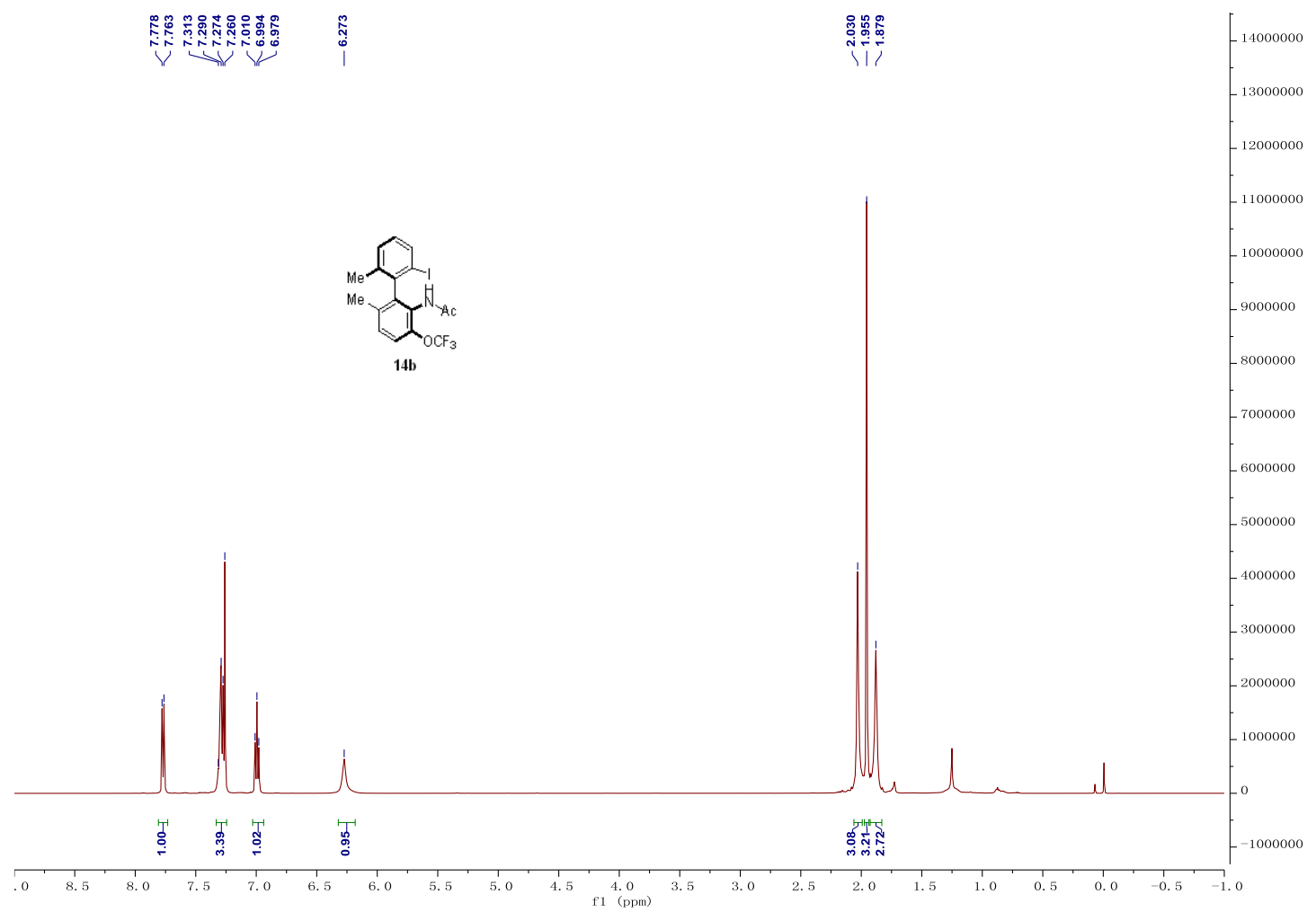

Figure S68. ${ }^{1} \mathrm{H}$ NMR spectra $\left(500 \mathrm{MHz}, \mathrm{CDCl}_{3}\right)$ of $\mathbf{1 4 b}$ 


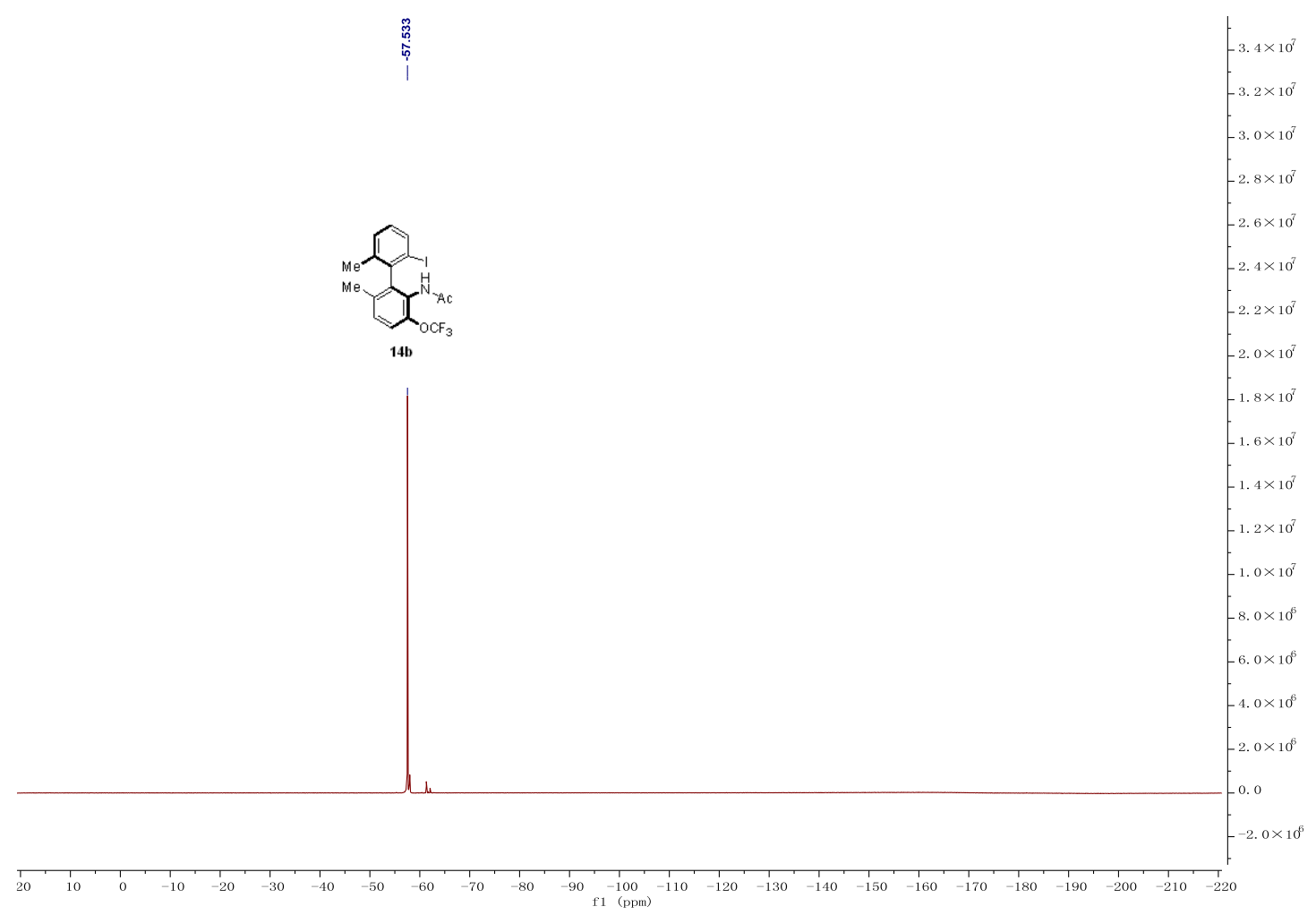

Figure S69. ${ }^{19} \mathrm{~F}$ NMR spectra $\left(471 \mathrm{MHz}, \mathrm{CDCl}_{3}\right)$ of $\mathbf{1 4 b}$

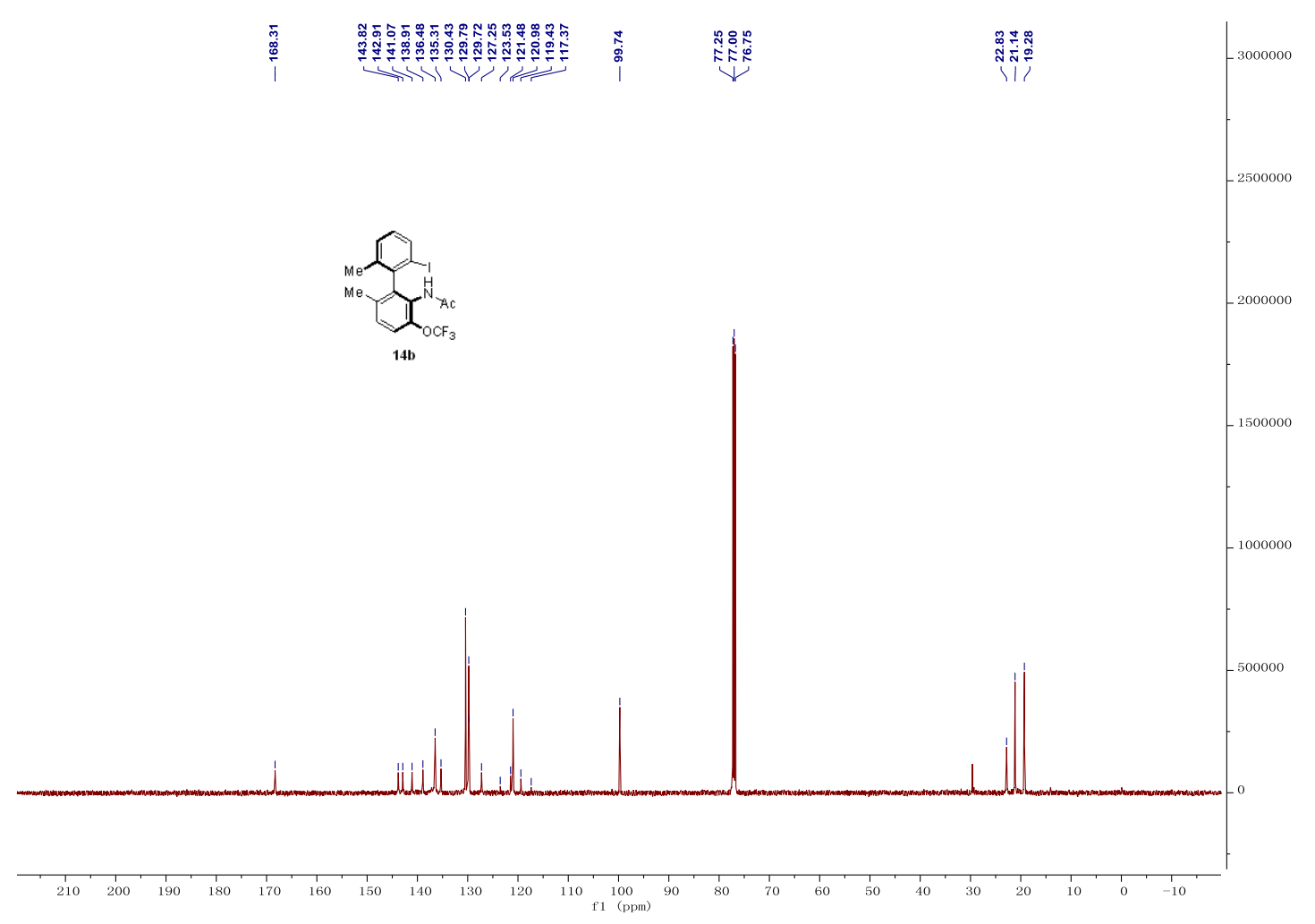

Figure S70. ${ }^{13} \mathrm{C}$ NMR spectra $\left(126 \mathrm{MHz}, \mathrm{CDCl}_{3}\right)$ of $\mathbf{1 4 b}$ 


\section{Copies of HPLC Traces}

\section{<Chromatogram>}

$\mathrm{mV} \quad$ Chiralpak AD-H, isopropanol/hexane $=10: 90$, flow: $1.0 \mathrm{~mL} / \mathrm{min}, \lambda=254 \mathrm{~nm}$

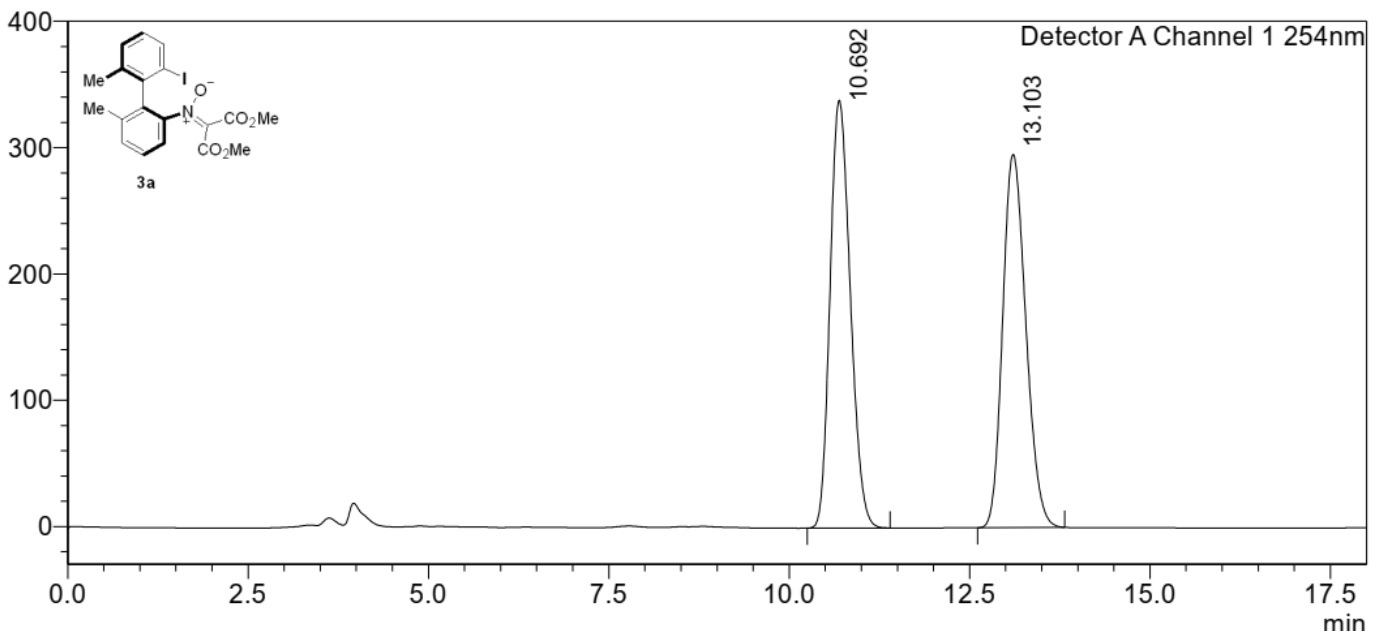

<Peak Table>

Detector A Channel $1254 \mathrm{~nm}$

\begin{tabular}{|r|r|r|r|r|r|r|r|}
\hline Peak\# & Ret. Time & \multicolumn{1}{c|}{ Area } & Height & Conc. & Unit & Mark & Name \\
\hline 1 & 10.692 & 6618309 & 338765 & 50.058 & & $\mathrm{M}$ & \\
\hline 2 & 13.103 & 6602880 & 295523 & 49.942 & & $\mathrm{M}$ & \\
\hline Total & & 13221189 & 634288 & & & & \\
\hline
\end{tabular}

Figure S71. HPLC spectra of 3a-rac

\section{$<$ Chromatogram>}

$\mathrm{mV}$ Chiralpak AD-H, isopropanol/hexane $=10: 90$, flow: $1.0 \mathrm{~mL} / \mathrm{min}, \lambda=254 \mathrm{~nm}$

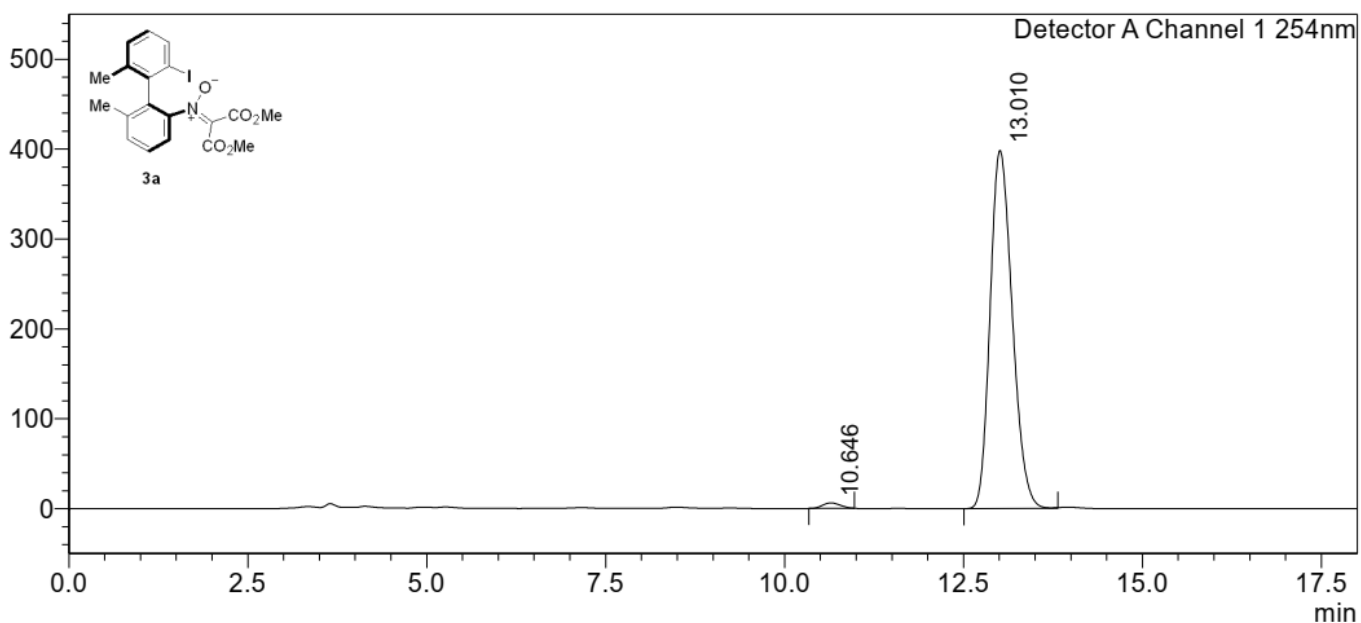

<Peak Table>

Detector A Channel $1254 \mathrm{~nm}$

\begin{tabular}{|r|r|r|r|r|r|r|r|}
\hline Peak\# & Ret. Time & \multicolumn{1}{c|}{ Area } & Height & Conc. & Unit & Mark & Name \\
\hline 1 & 10.646 & 106338 & 6131 & 1.224 & & $\mathrm{M}$ & \\
\hline 2 & 13.010 & 8582633 & 398602 & 98.776 & & $\mathrm{M}$ & \\
\hline Total & & 8688971 & 404733 & & & &
\end{tabular}

Figure S72. HPLC spectra of 3a 


\section{<Chromatogram>}

$\mathrm{mV}$

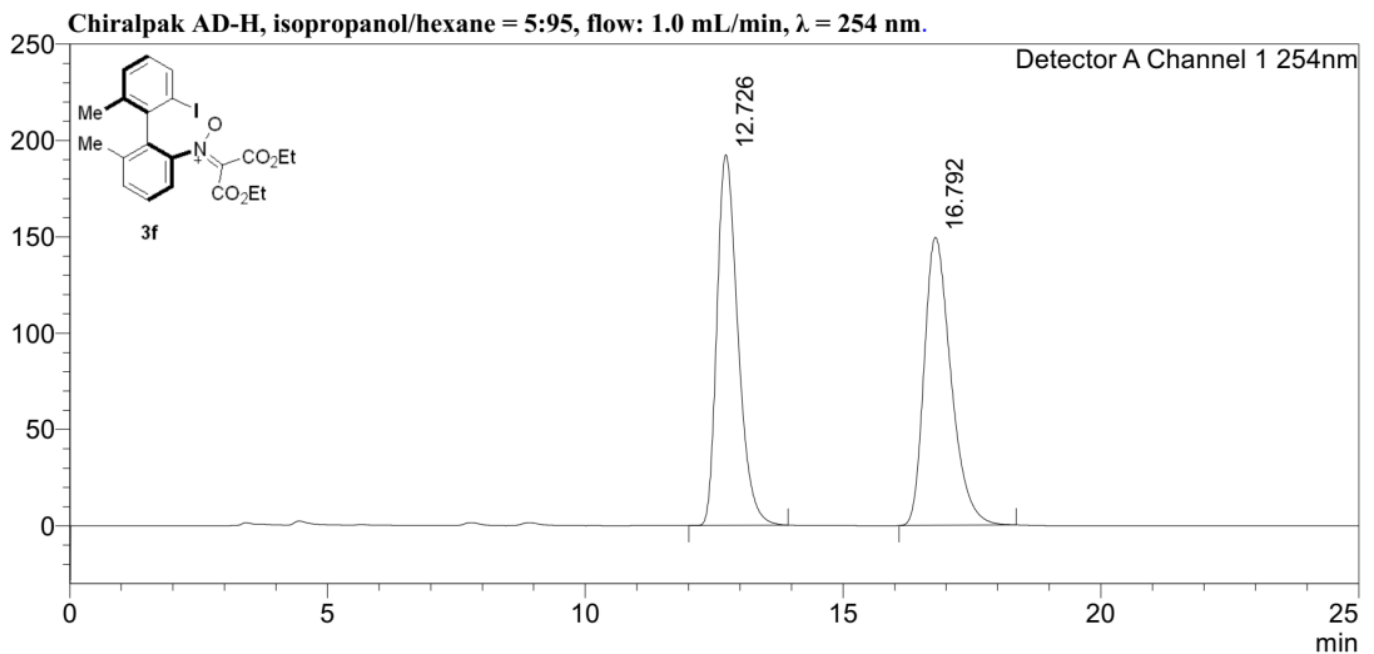

<Peak Table>

Detector A Channel $1254 n m$

\begin{tabular}{|r|r|r|r|r|r|r|r|}
\hline Peak\# & Ret. Time & \multicolumn{1}{|c|}{ Area } & Height & Conc. & Unit & Mark & Name \\
\hline 1 & 12.726 & 5432334 & 192438 & 50.129 & & $\mathrm{M}$ & \\
\hline 2 & 16.792 & 5404360 & 149490 & 49.871 & & $\mathrm{M}$ & \\
\hline Total & & 10836693 & 341928 & & & & \\
\hline
\end{tabular}

Figure S73. HPLC spectra of $\mathbf{3 f}$-rac

\section{$<$ Chromatogram $>$}

$\mathrm{mV}$

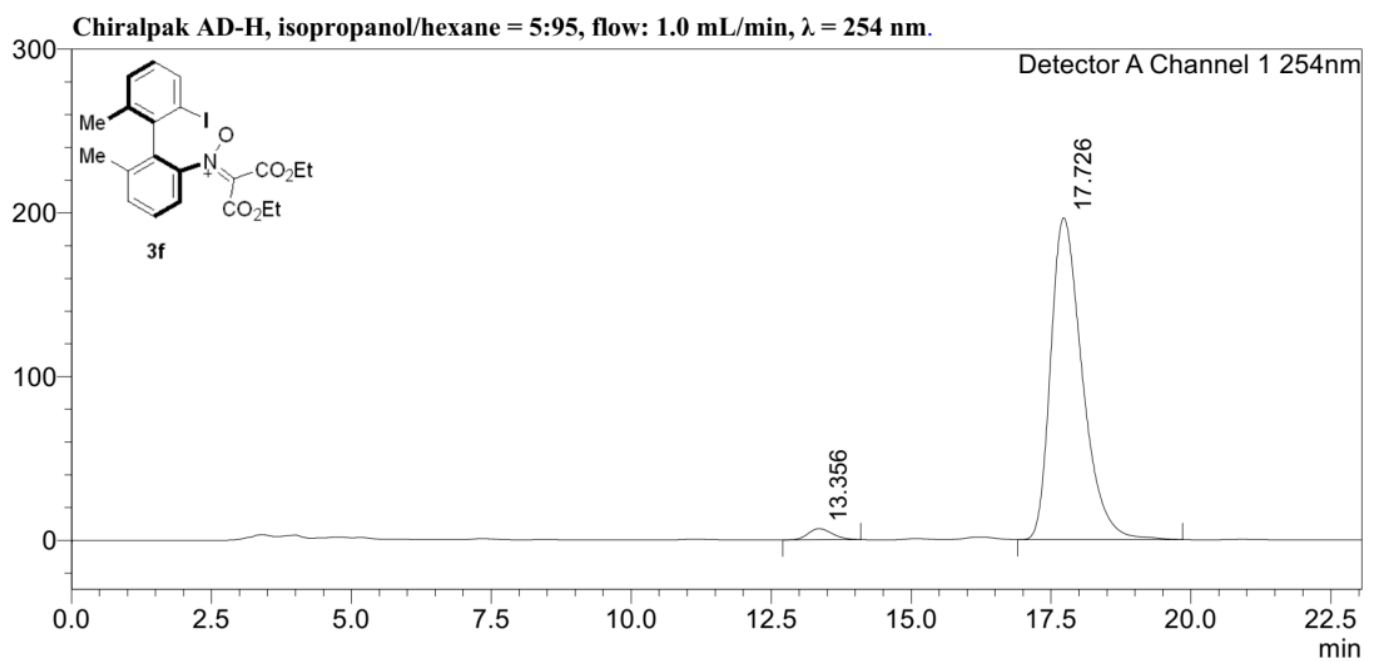

\section{<Peak Table>}

Detector A Channel $1254 \mathrm{~nm}$

\begin{tabular}{|r|r|r|r|r|r|r|r|}
\hline Peak\# & Ret. Time & \multicolumn{1}{|c|}{ Area } & Height & Conc. & Unit & Mark & Name \\
\hline 1 & 13.356 & 205862 & 6821 & 2.590 & & M & \\
\hline 2 & 17.726 & 7740965 & 196760 & 97.410 & & M & \\
\hline Total & & 7946827 & 203581 & & & & \\
\hline
\end{tabular}

Figure S74. HPLC spectra of $\mathbf{3 f}$ 


\section{<Chromatogram>}

$\mathrm{mV}$

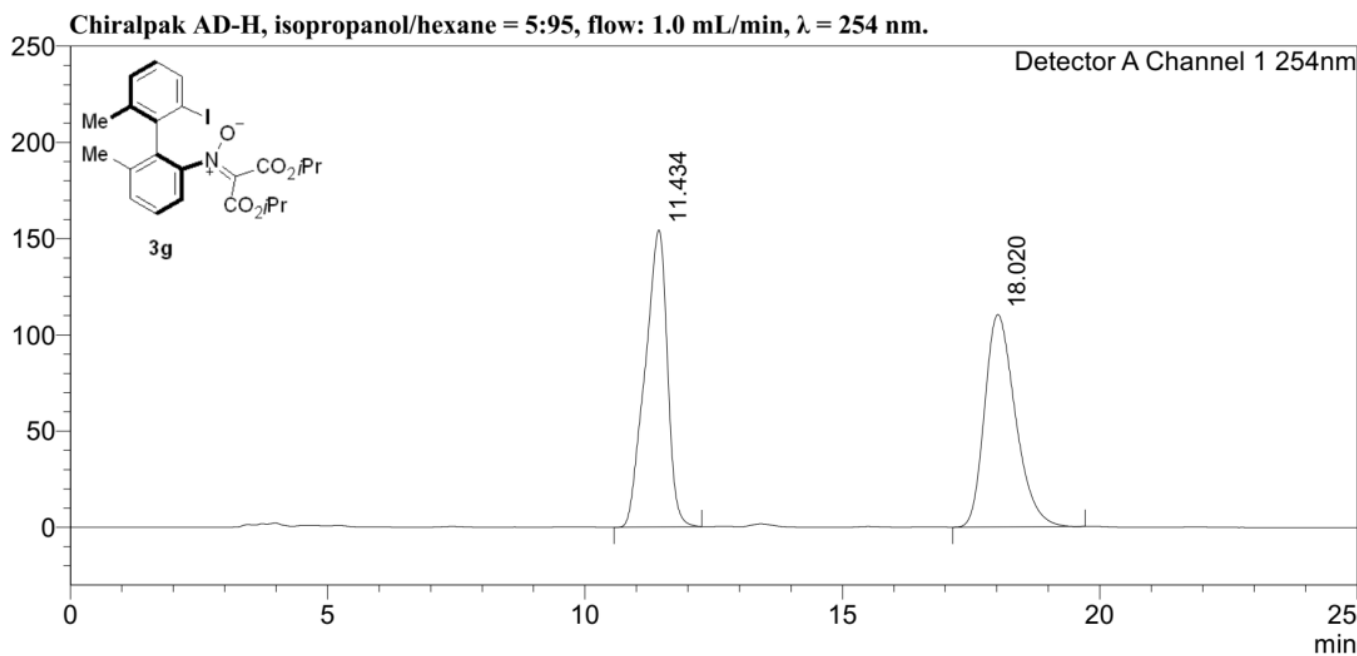

<Peak Table>

Detector A Channel 1 254nm

\begin{tabular}{|r|r|r|r|r|r|r|r|}
\hline Peak\# Ret. Time & Area & Height & Conc. & Unit & Mark & Name \\
\hline 1 & 11.434 & 4628563 & 154349 & 50.191 & & $\mathrm{M}$ & \\
\hline 2 & 18.020 & 4593287 & 110351 & 49.809 & & $\mathrm{M}$ & \\
\hline Total & & 9221850 & 264699 & & & & \\
\hline
\end{tabular}

Figure S75. HPLC spectra of 3g-rac

\section{$<$ Chromatogram $>$}

$\mathrm{mV}$

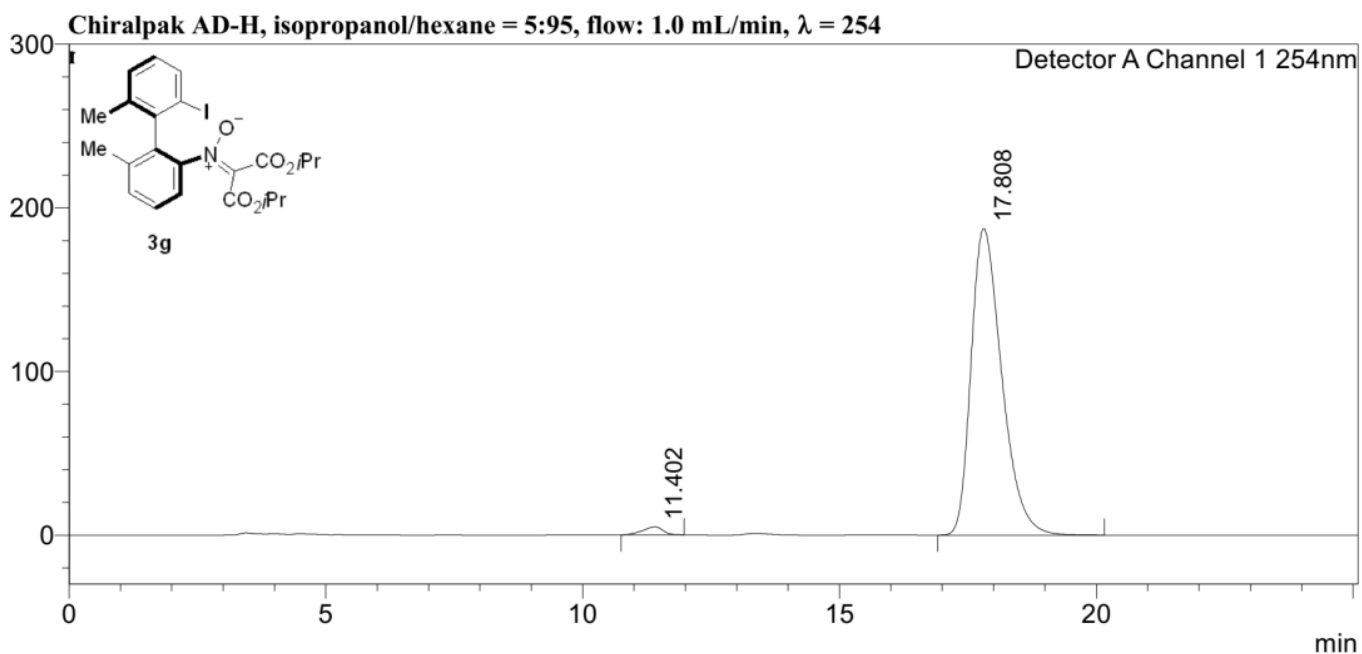

<Peak Table>

Detector A Channel $1254 \mathrm{~nm}$

\begin{tabular}{|r|r|r|r|r|r|r|r|}
\hline Peak\# Ret. Time & \multicolumn{1}{|c|}{ Area } & \multicolumn{1}{|c|}{ Height } & \multicolumn{1}{c|}{ Conc. } & Unit & Mark & Name \\
\hline 1 & 11.402 & 133294 & 4909 & 1.683 & & $\mathrm{M}$ & \\
\hline 2 & 17.808 & 7788412 & 187448 & 98.317 & & $\mathrm{M}$ & \\
\hline Total & & 7921706 & 192356 & & & & \\
\hline
\end{tabular}

Figure S76. HPLC spectra of $\mathbf{3 g}$ 
<Chromatogram>

$\mathrm{mV}$

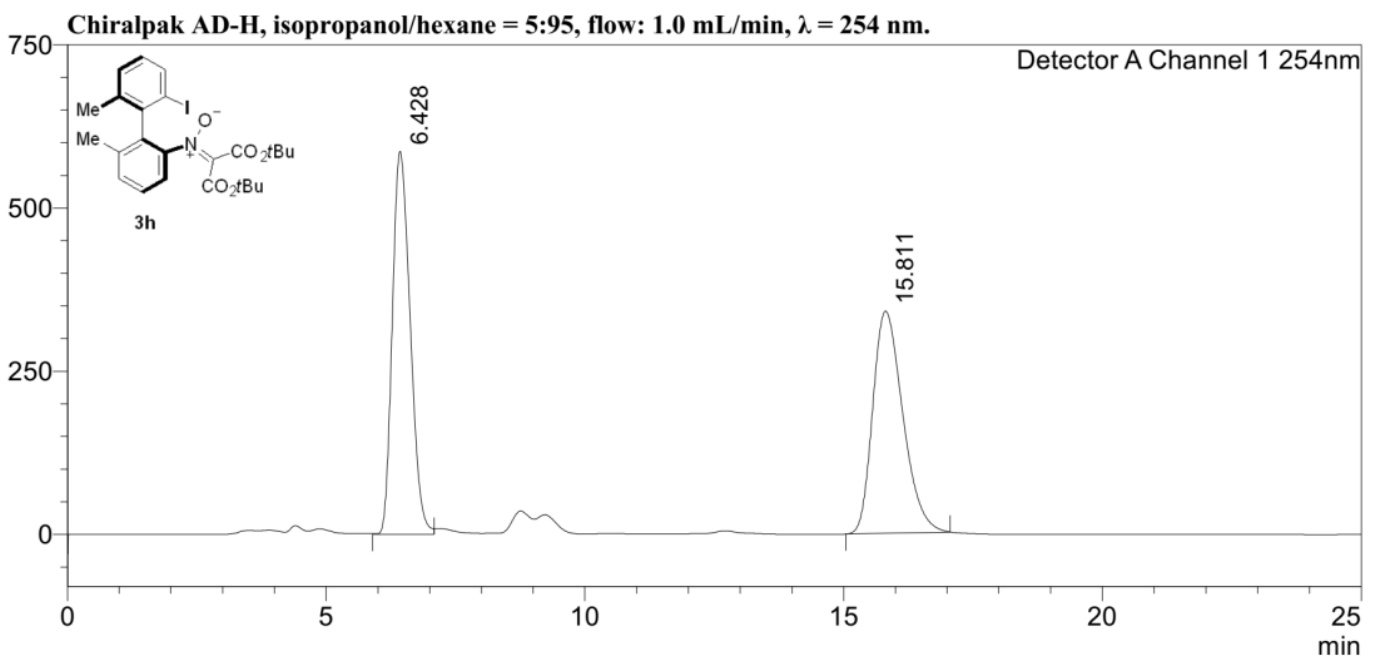

<Peak Table>

Detector A Channel $1254 \mathrm{~nm}$

\begin{tabular}{|r|r|r|r|r|r|r|r|}
\hline Peak\# Ret. Time & \multicolumn{1}{|c|}{ Area } & Height & \multicolumn{1}{c|}{ Conc. } & Unit & Mark & Name \\
\hline 1 & 6.428 & 14032171 & 586231 & 50.501 & & $\mathrm{M}$ & \\
\hline 2 & 15.811 & 13753986 & 340335 & 49.499 & & $\mathrm{M}$ & \\
\hline Total & & 27786157 & 926566 & & & & \\
\hline
\end{tabular}

Figure S77. HPLC spectra of $\mathbf{3 h}$-rac

\section{$<$ Chromatogram $>$}

$\mathrm{mV}$

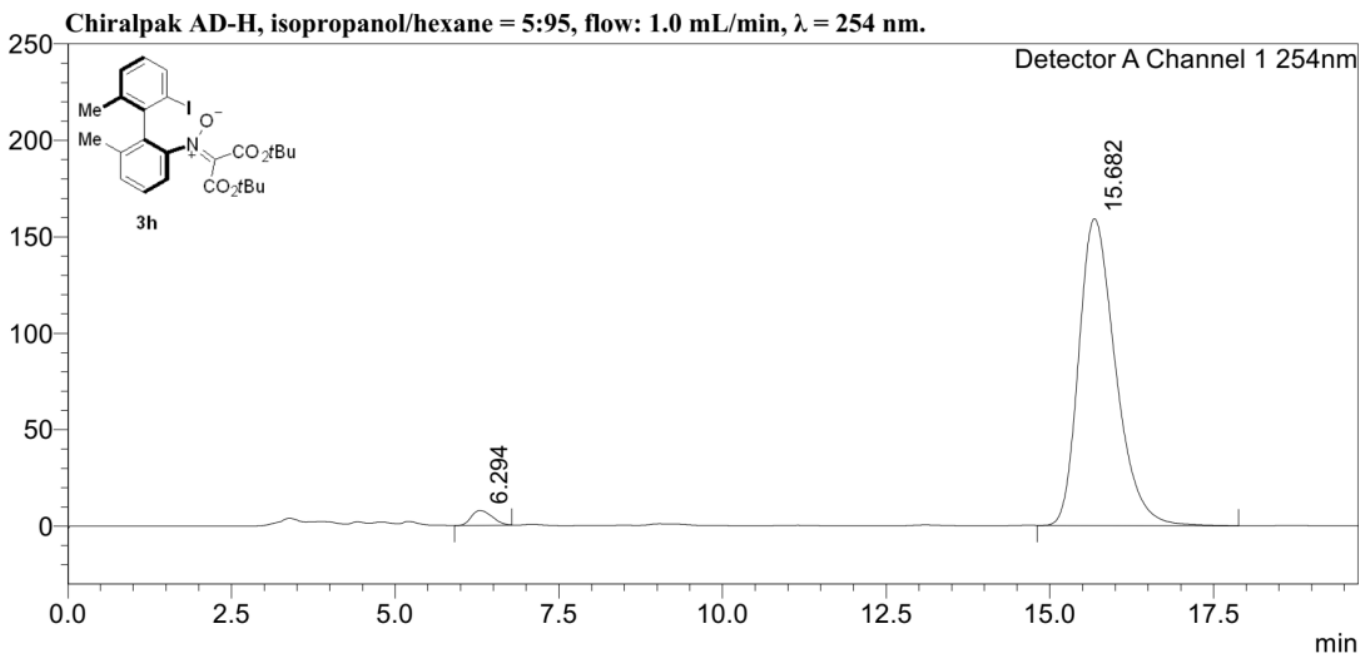

<Peak Table>

Detector A Channel $1254 \mathrm{~nm}$

\begin{tabular}{|r|r|r|r|r|r|r|r|}
\hline Peak\# Ret. Time & \multicolumn{1}{|c|}{ Area } & Height & Conc. & Unit & Mark & Name \\
\hline 1 & 6.294 & 167729 & 7689 & 2.634 & & $\mathrm{M}$ & \\
\hline 2 & 15.682 & 6200916 & 159102 & 97.366 & & $\mathrm{M}$ & \\
\hline Total & & 6368644 & 166791 & & & & \\
\hline
\end{tabular}

Figure S78. HPLC spectra of $\mathbf{3 h}$ 


\section{<Chromatogram>}

$\mathrm{mV}$

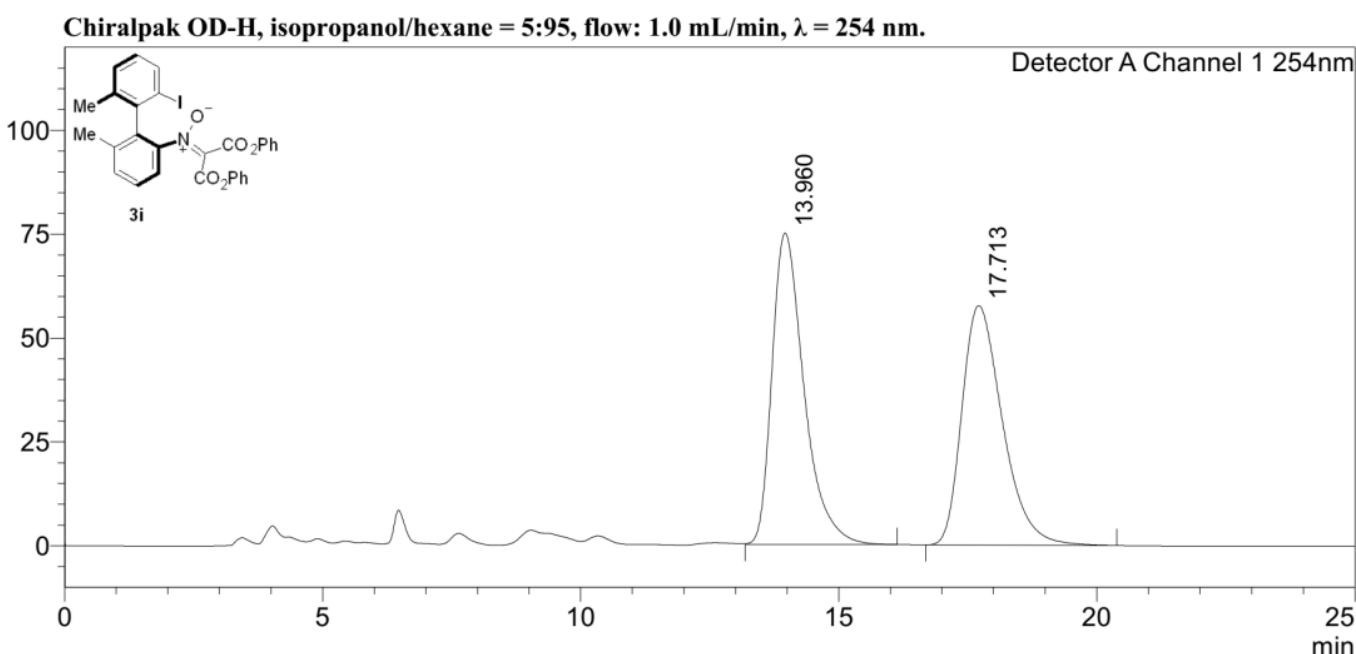

<Peak Table>

Detector A Channel $1254 \mathrm{~nm}$

\begin{tabular}{|r|r|r|r|r|r|r|r|}
\hline Peak\# & Ret. Time & Area & Height & \multicolumn{1}{c|}{ Conc. } & Unit & Mark & Name \\
\hline 1 & 13.960 & 3237077 & 75006 & 50.713 & & $\mathrm{M}$ & \\
\hline 2 & 17.713 & 3146082 & 57646 & 49.287 & & $\mathrm{M}$ & \\
\hline Total & & 6383159 & 132652 & & & & \\
\hline
\end{tabular}

Figure S79. HPLC spectra of 3i-rac

\section{<Chromatogram>}

$\mathrm{mV}$

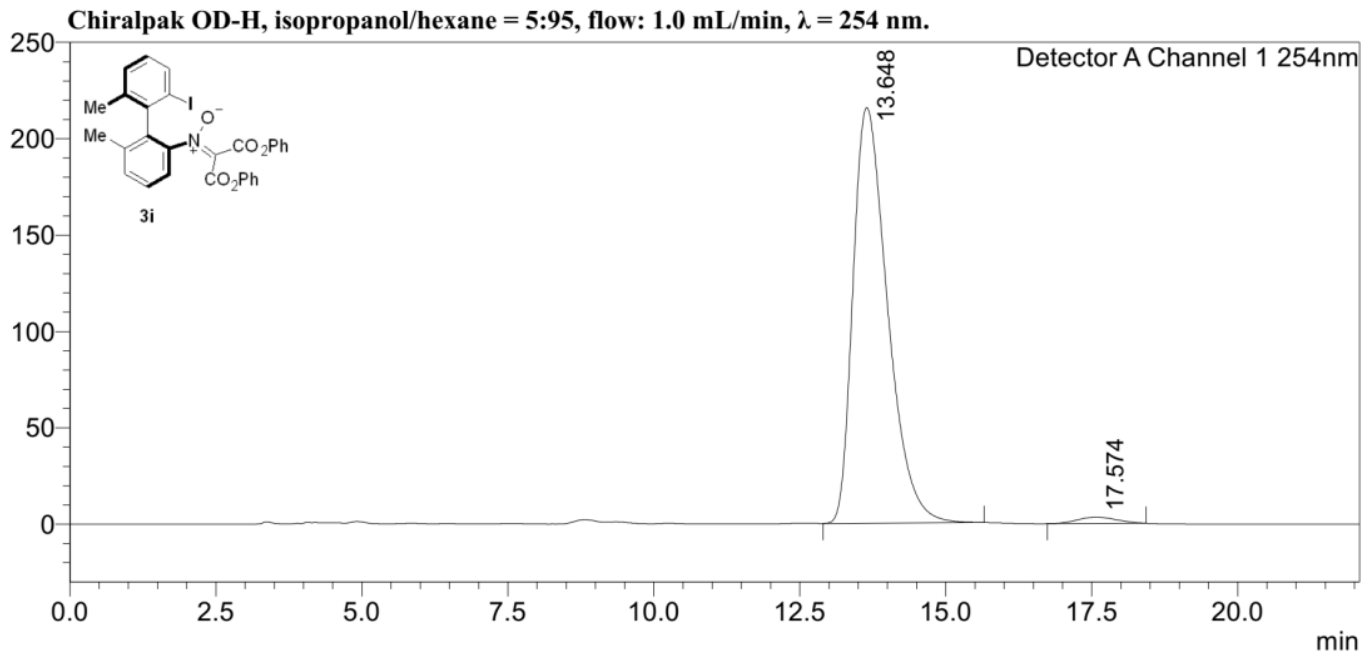

<Peak Table>

Detector A Channel $1254 \mathrm{~nm}$

\begin{tabular}{|r|r|r|r|r|r|r|r|}
\hline Peak\# Ret. Time & \multicolumn{1}{|c|}{ Area } & \multicolumn{1}{|c|}{ Height } & \multicolumn{1}{c|}{ Conc. } & Unit & Mark & Name \\
\hline 1 & 13.648 & 8859470 & 215639 & 98.283 & & $\mathrm{M}$ & \\
\hline 2 & 17.574 & 154793 & 3308 & 1.717 & & $\mathrm{M}$ & \\
\hline Total & & 9014264 & 218947 & & & & \\
\end{tabular}

Figure S80. HPLC spectra of $\mathbf{3 i}$ 

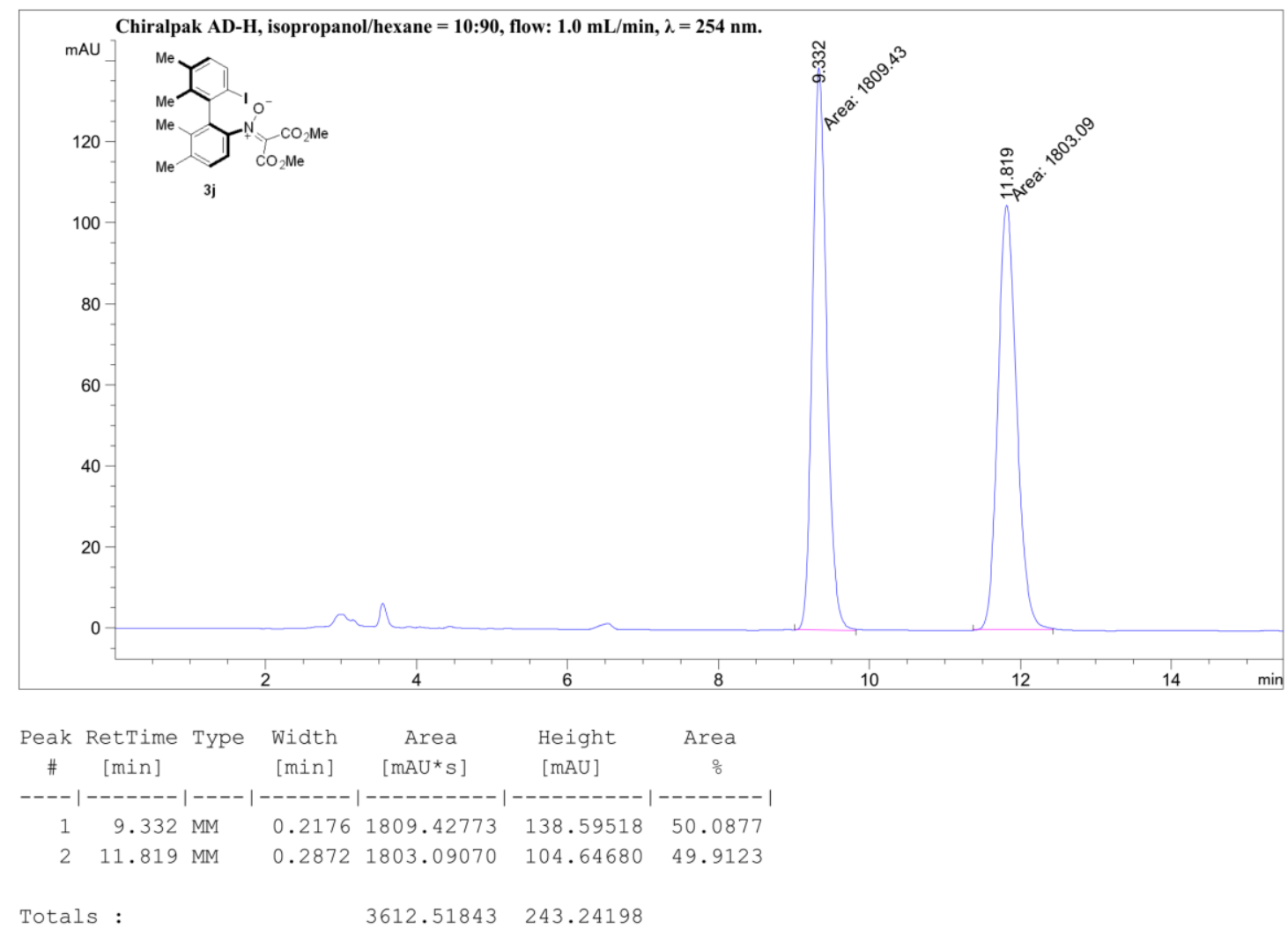

Figure S81. HPLC spectra of $\mathbf{3 j}$-rac

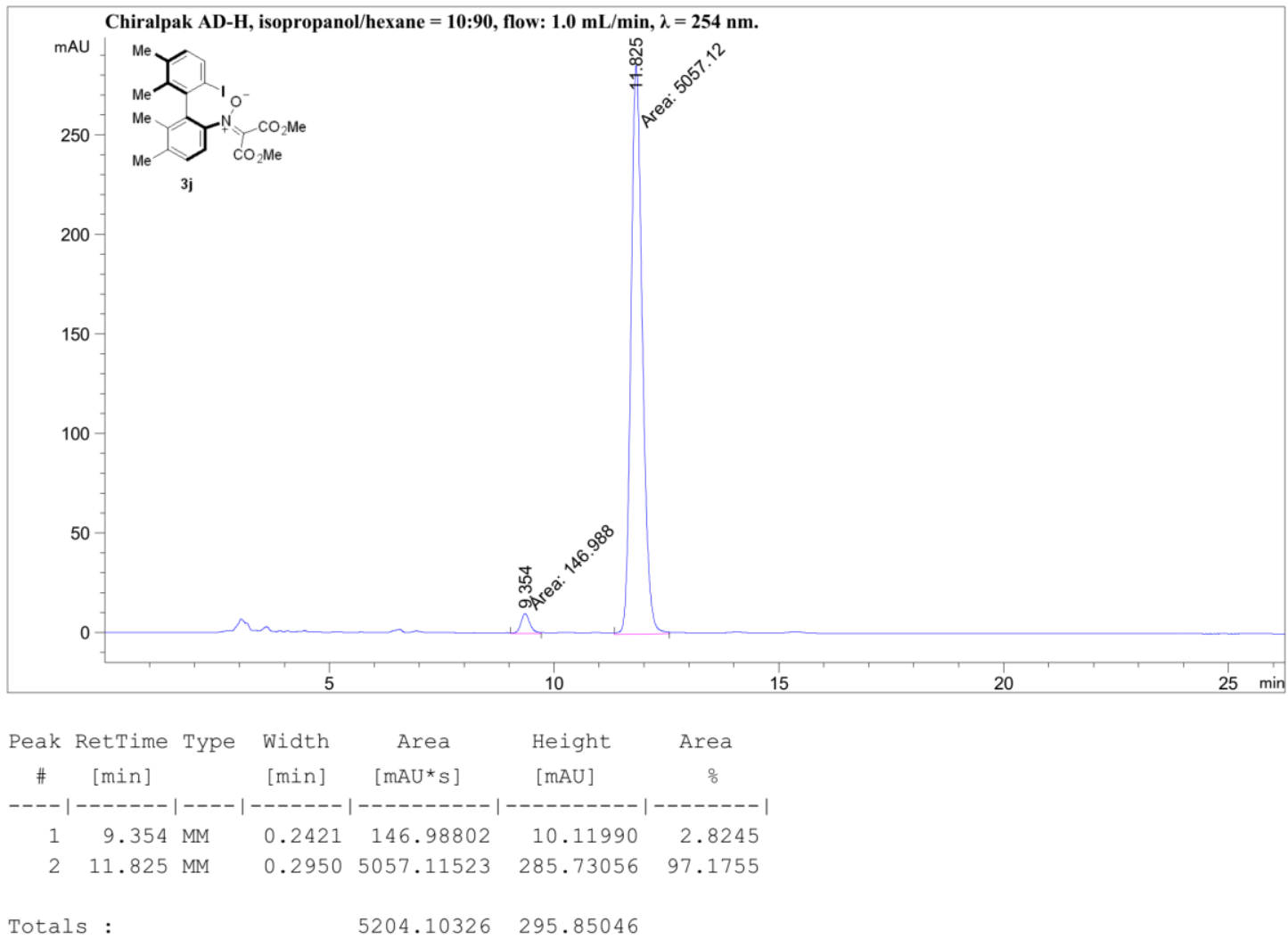

Figure S82. HPLC spectra of $\mathbf{3 j}$ 


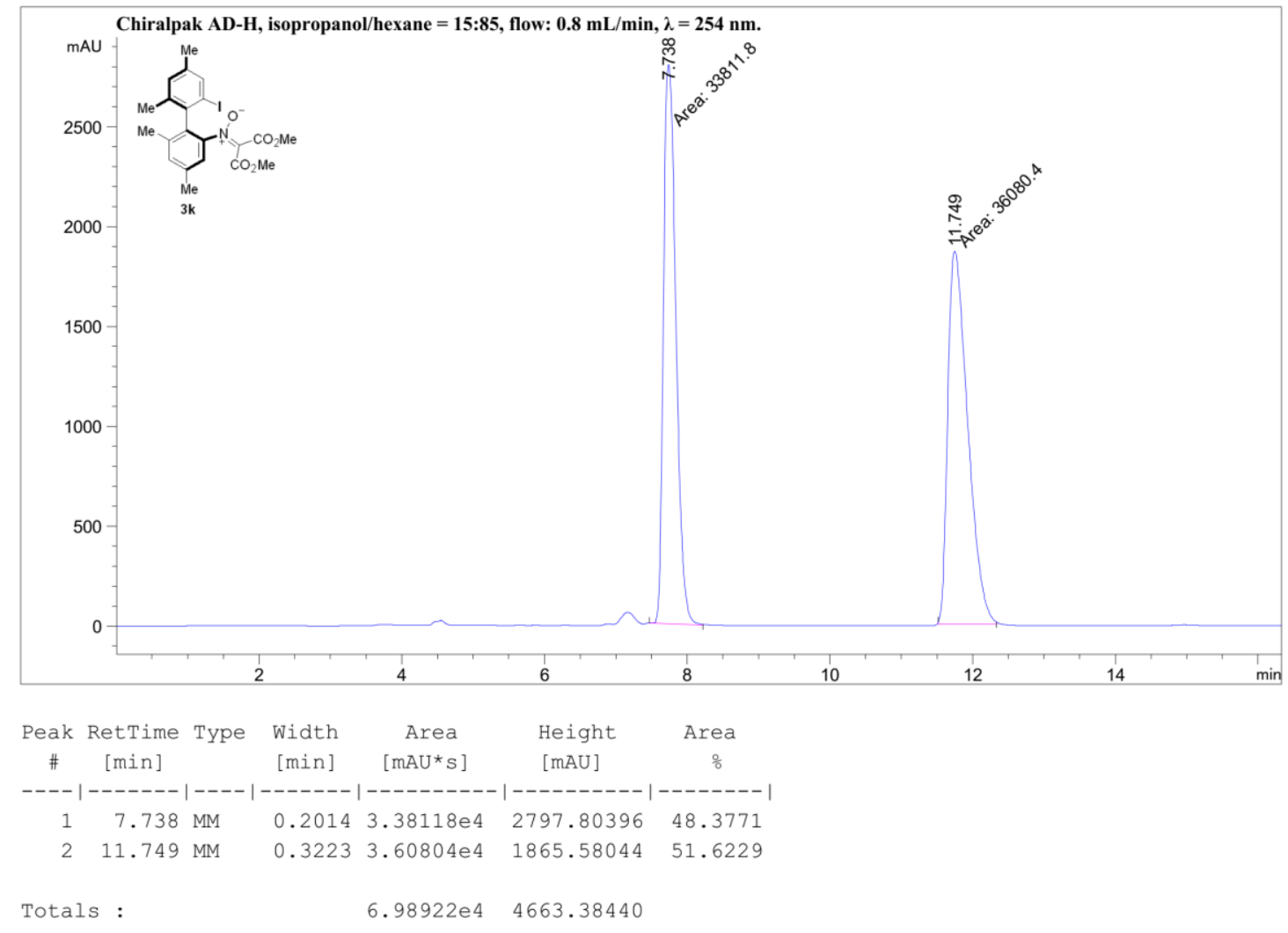

Figure S83. HPLC spectra of 3k-rac

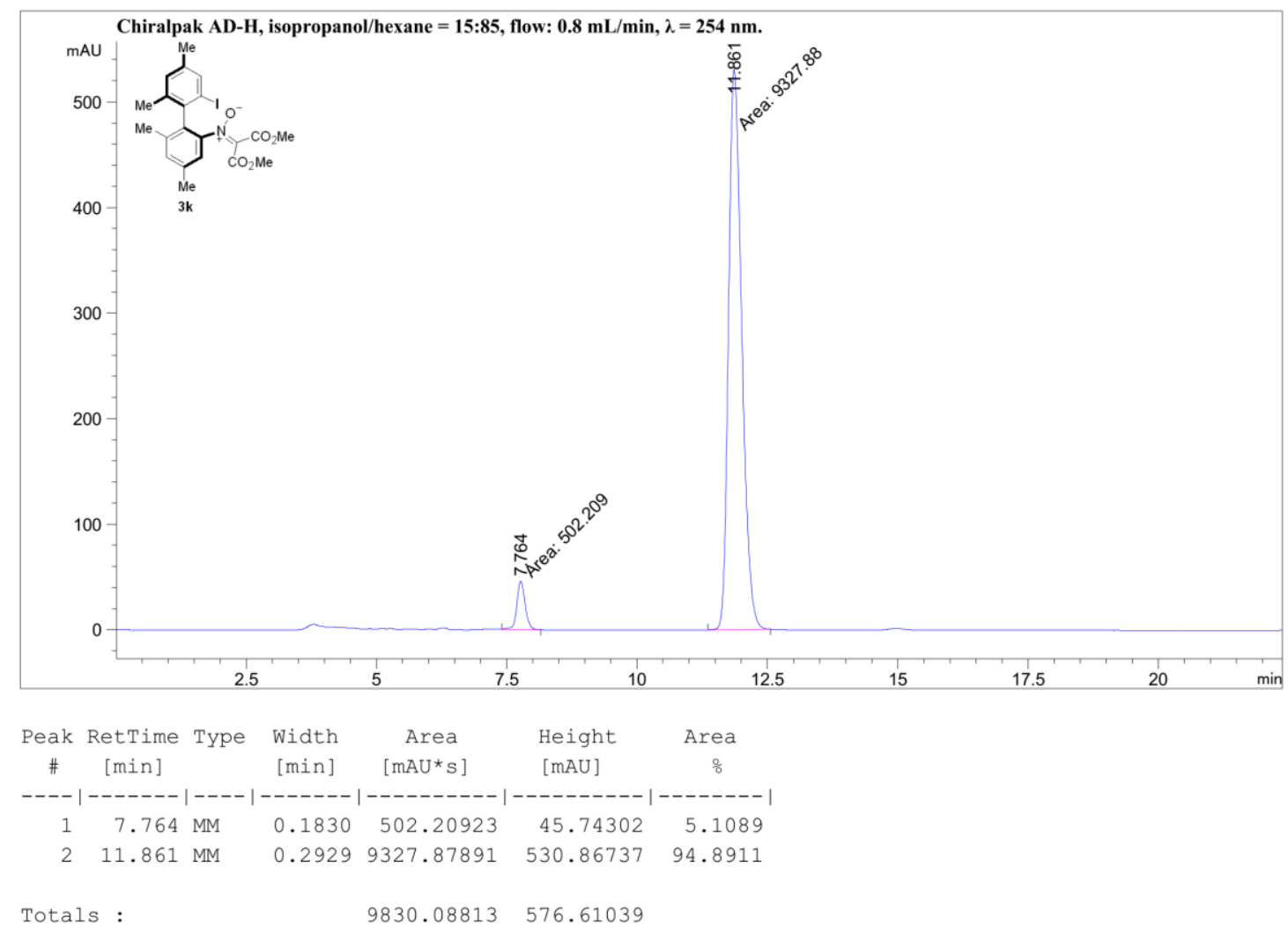

Figure S84. HPLC spectra of 3k 


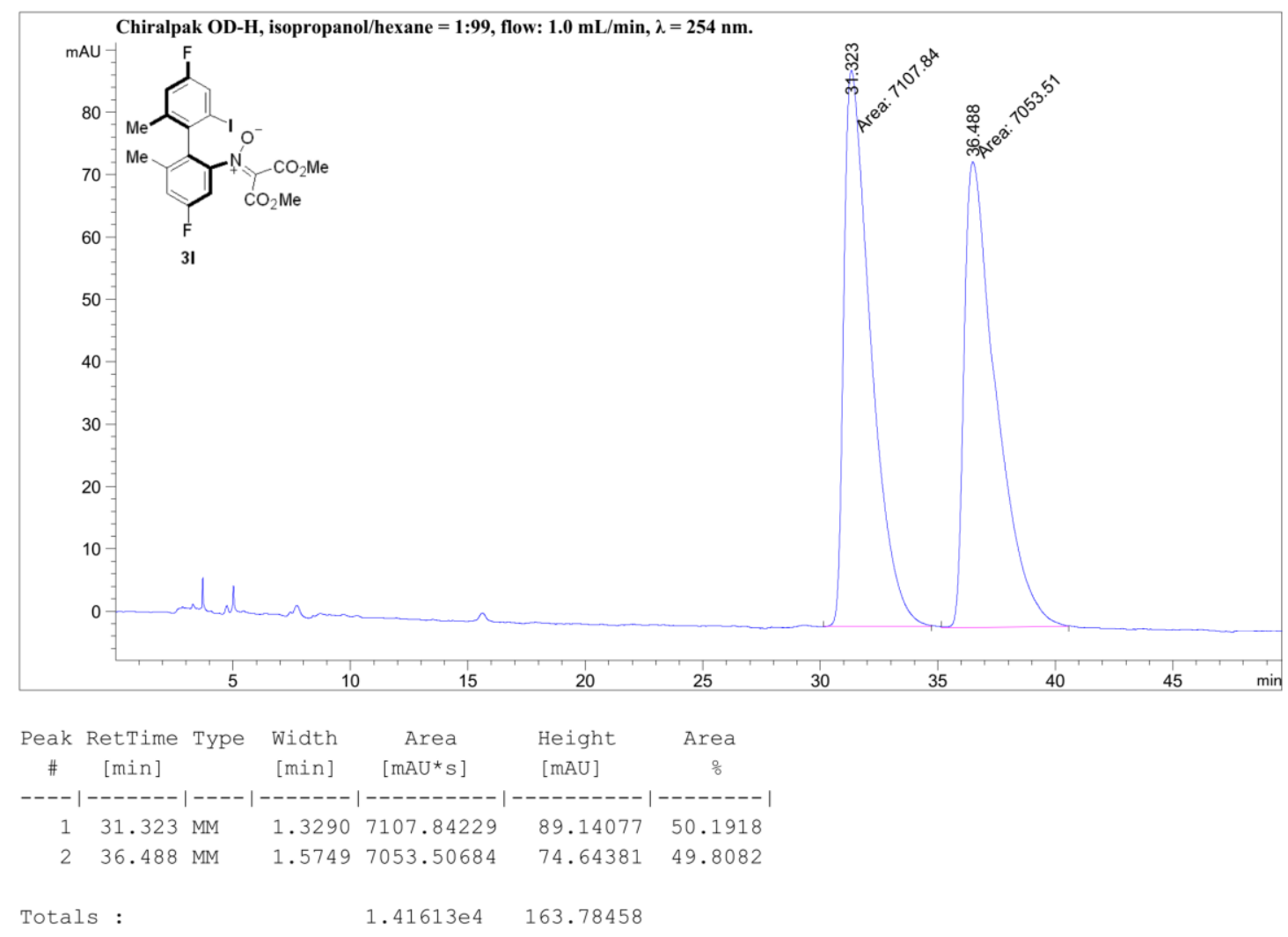

Figure S85. HPLC spectra of 3l-rac

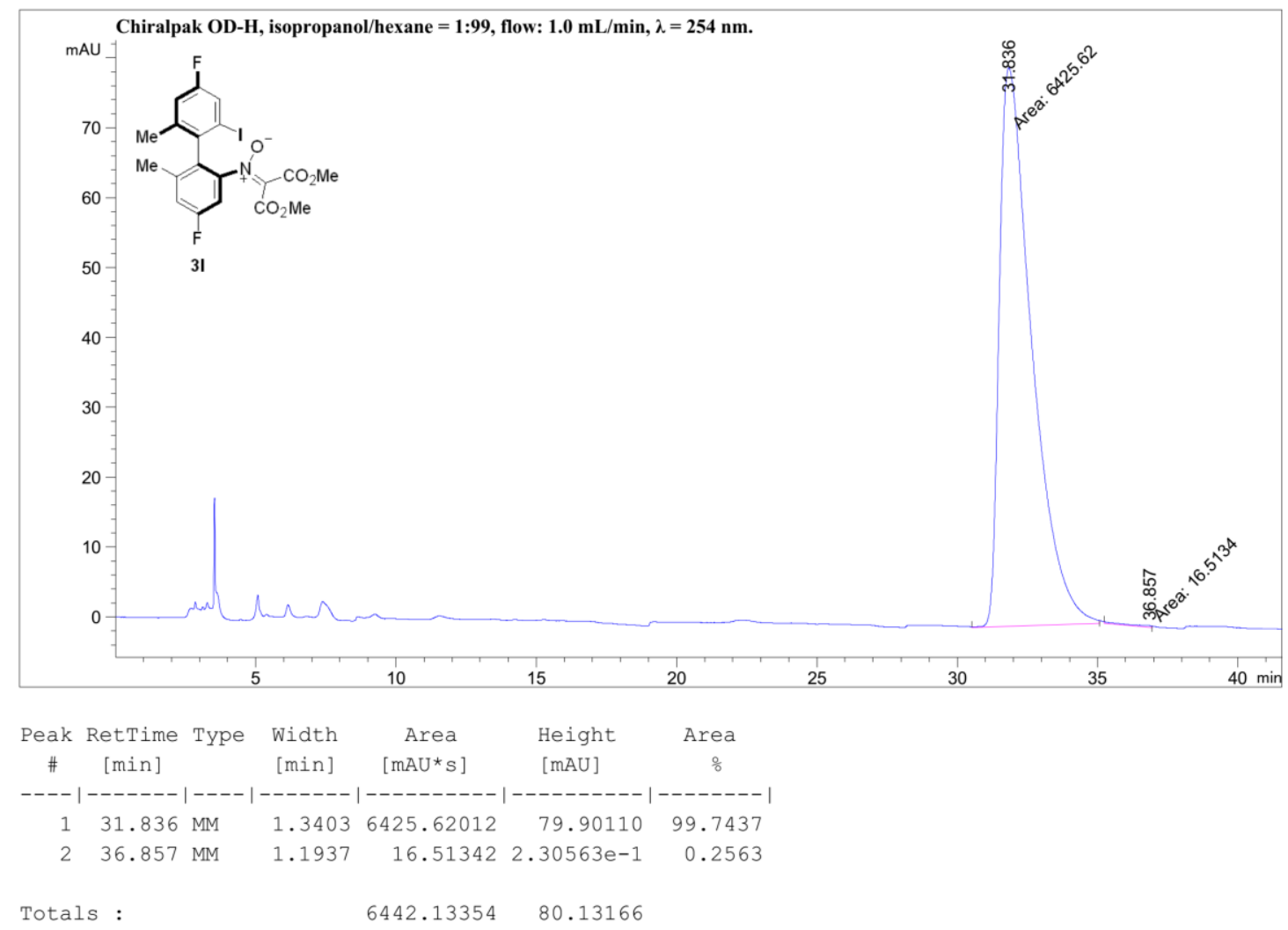

Figure S86. HPLC spectra of $\mathbf{3 1}$ 


\section{$<$ Chromatogram $>$}

$\mathrm{mV}$

Chiralpak IA, isopropanol $/$ hexane $=30: 70$, flow: $1.0 \mathrm{~mL} / \mathrm{min}, \lambda=254 \mathrm{~nm}$.

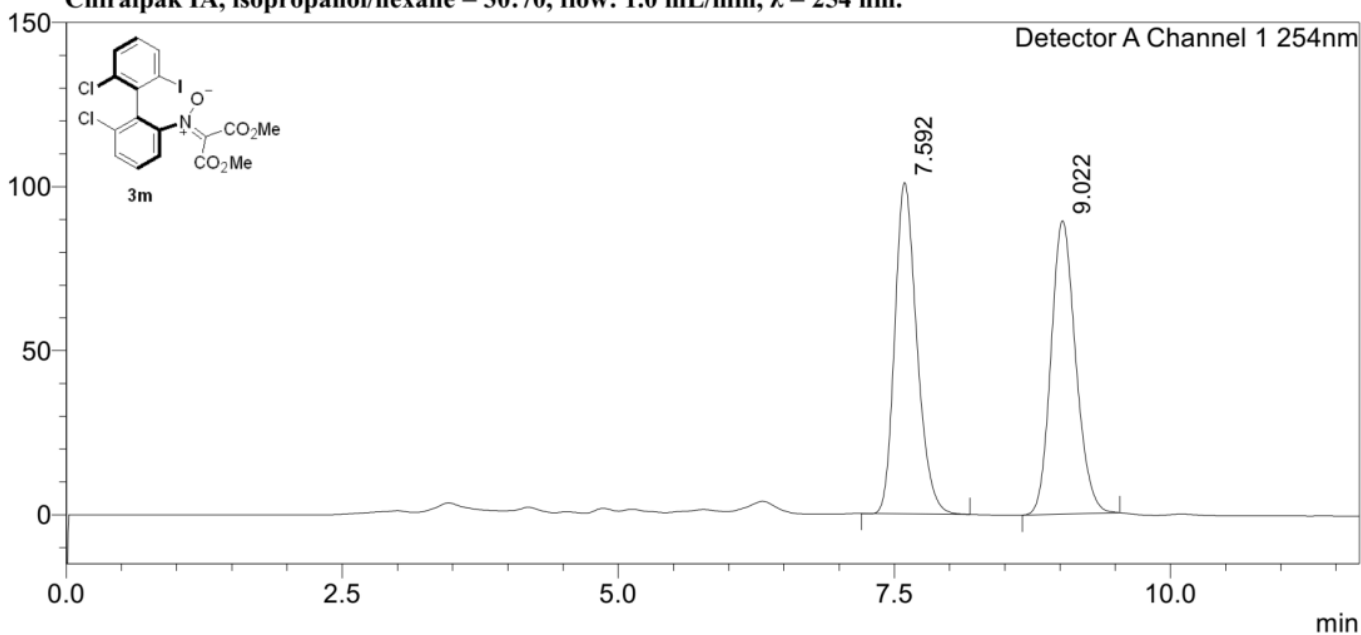

<Peak Table>

Detector A Channel $1254 \mathrm{~nm}$

\begin{tabular}{|r|r|r|r|r|r|r|r|}
\hline Peak\# Ret. Time & \multicolumn{1}{|c|}{ Area } & Height & Conc. & Unit & Mark & Name \\
\hline 1 & 7.592 & 1406214 & 100995 & 50.276 & & $\mathrm{M}$ & \\
\hline 2 & 9.022 & 1390797 & 89463 & 49.724 & & $\mathrm{M}$ & \\
\hline Total & & 2797011 & 190458 & & & & \\
\hline
\end{tabular}

Figure S87. HPLC spectra of 3m-rac

\section{$<$ Chromatogram>}

$\mathrm{mV}$

Chiralpak IA, isopropanol $/$ hexane $=30: 70$, flow: $1.0 \mathrm{~mL} / \mathrm{min}, \lambda=254 \mathrm{~nm}$.

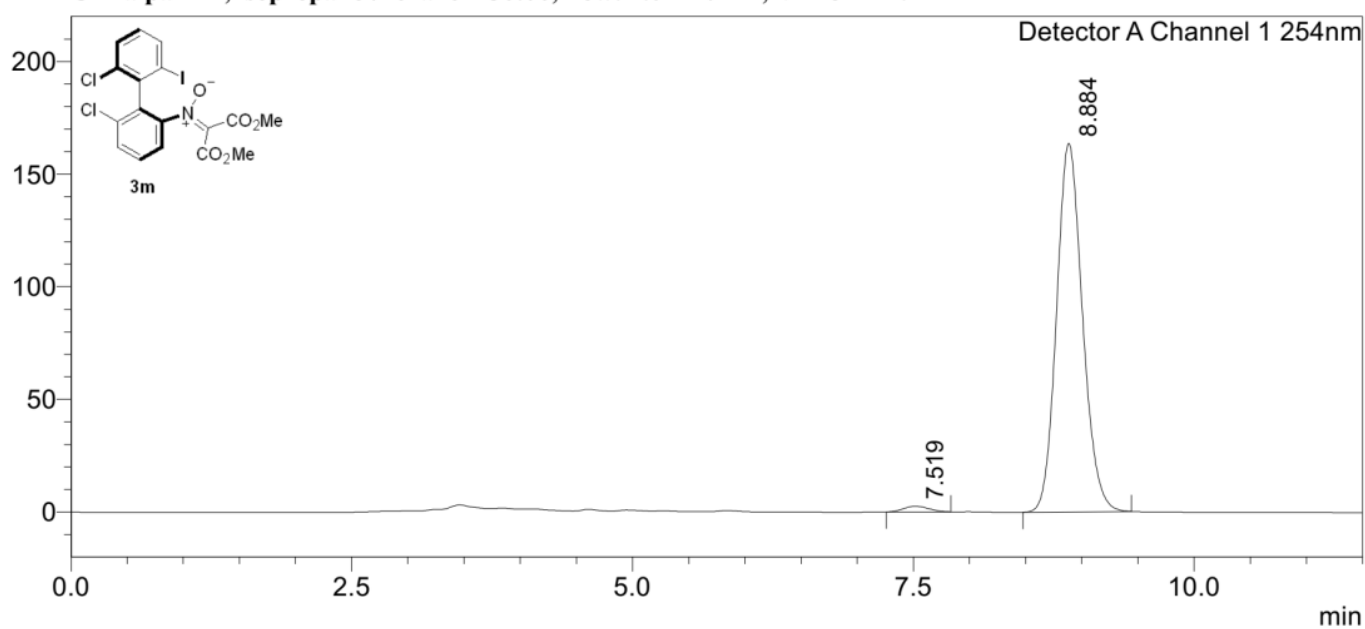

<Peak Table>

Detector A Channel $1254 \mathrm{~nm}$

\begin{tabular}{|r|r|r|r|r|r|r|r|}
\hline Peak\# Ret. Time & \multicolumn{1}{|c|}{ Area } & Height & \multicolumn{1}{|c|}{ Conc. } & Unit & Mark & Name \\
\hline 1 & 7.519 & 39346 & 2514 & 1.476 & & $\mathrm{M}$ & \\
\hline 2 & 8.884 & 2626881 & 163608 & 98.524 & & $\mathrm{M}$ & \\
\hline Total & & 2666227 & 166122 & & & & \\
\hline
\end{tabular}

Figure S88. HPLC spectra of 3m 
$<$ Chromatogram $>$

$\mathrm{mV}$

Chiralpak IA, isopropanol/hexane $=30: 70$, flow: $1.0 \mathrm{~mL} / \mathrm{min}, \lambda=254 \mathrm{~nm}$.

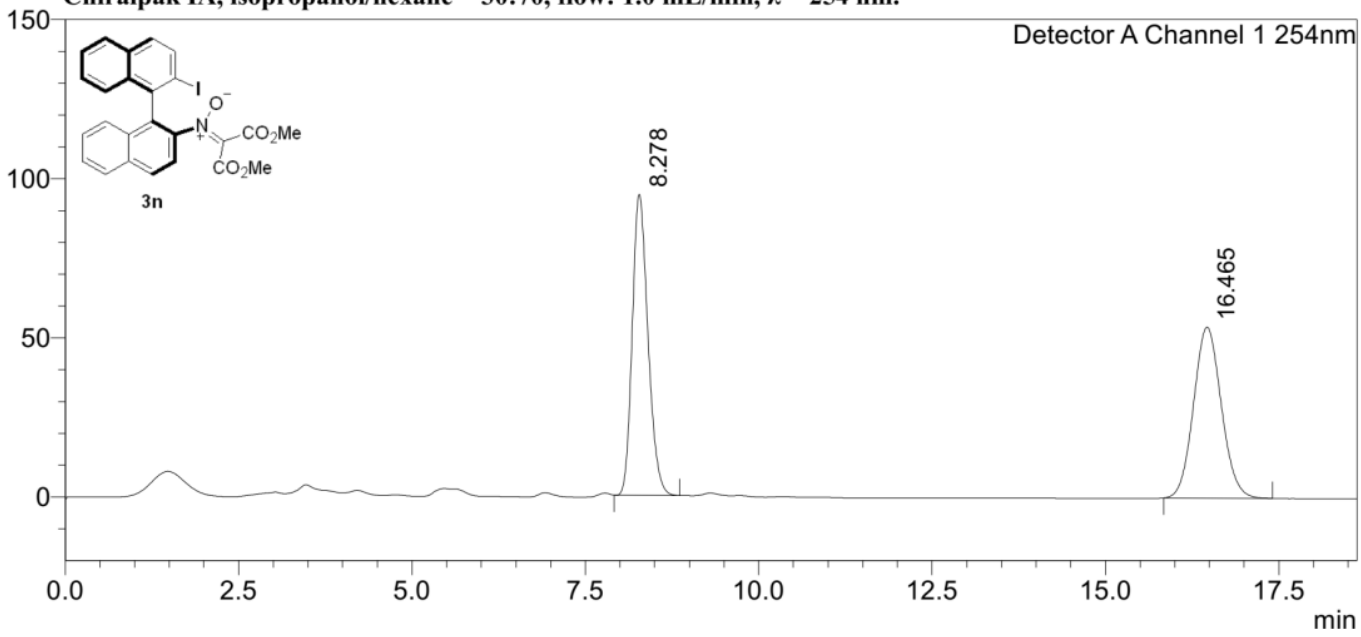

<Peak Table>

Detector A Channel $1254 \mathrm{~nm}$

\begin{tabular}{|r|r|r|r|r|r|r|r|}
\hline Peak\# & Ret. Time & Area & Height & Conc. & Unit & Mark & Name \\
\hline 1 & 8.278 & 1490918 & 94559 & 49.916 & & $\mathrm{M}$ & \\
\hline 2 & 16.465 & 1495918 & 53736 & 50.084 & & $\mathrm{M}$ & \\
\hline Total & & 2986835 & 148296 & & & & \\
\hline
\end{tabular}

Figure S89. HPLC spectra of 3n-rac

\section{$<$ Chromatogram $>$}

$\mathrm{mV}$

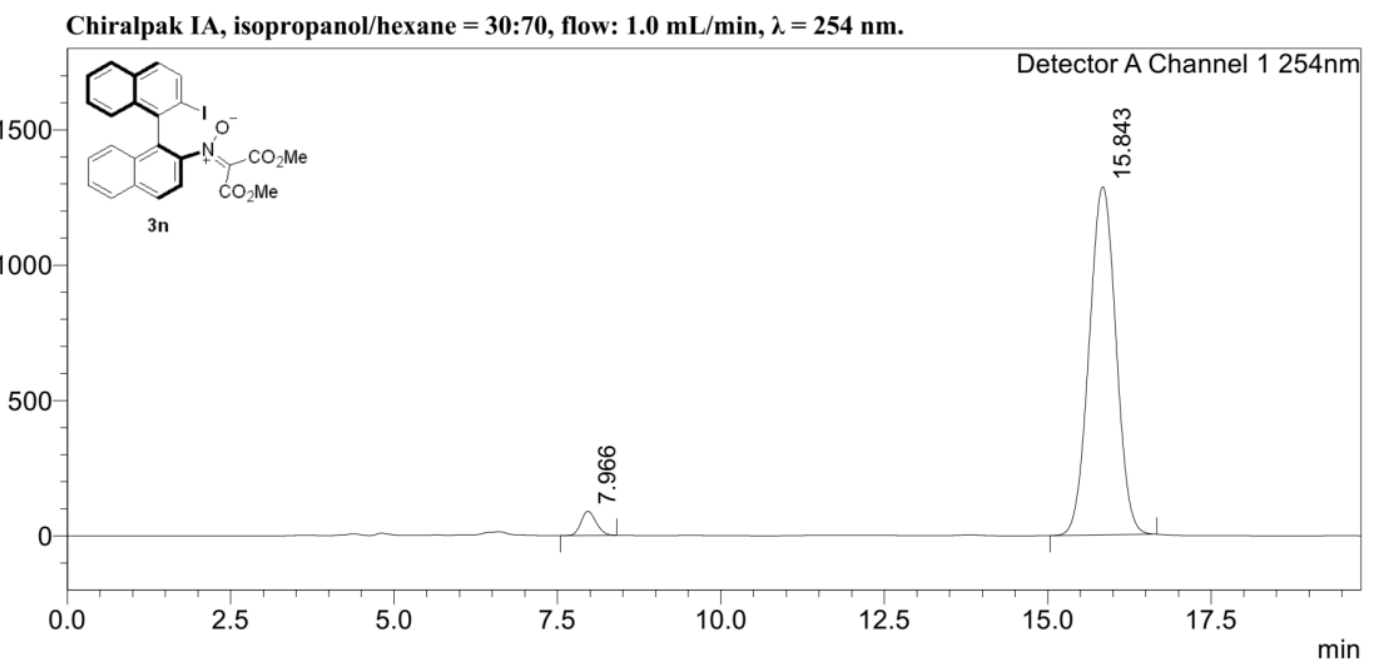

<Peak Table>

Detector A Channel 1 254nm Peak\# Ret. Time Area

\begin{tabular}{|r|r|r|r|r|r|r|r|}
\hline Peak\# & Ret. Time & \multicolumn{1}{|c|}{ Area } & Height & \multicolumn{1}{c|}{ Conc. } & Unit & Mark & Name \\
\hline 1 & 7.966 & 1422512 & 89368 & 3.730 & & $\mathrm{M}$ & \\
\hline 2 & 15.843 & 36709623 & 1287292 & 96.270 & & $\mathrm{M}$ & \\
\hline Total & & 38132135 & 1376660 & & & & \\
\hline
\end{tabular}

Figure S90. HPLC spectra of 3n 


\section{$<$ Chromatogram>}

$\mathrm{mV}$

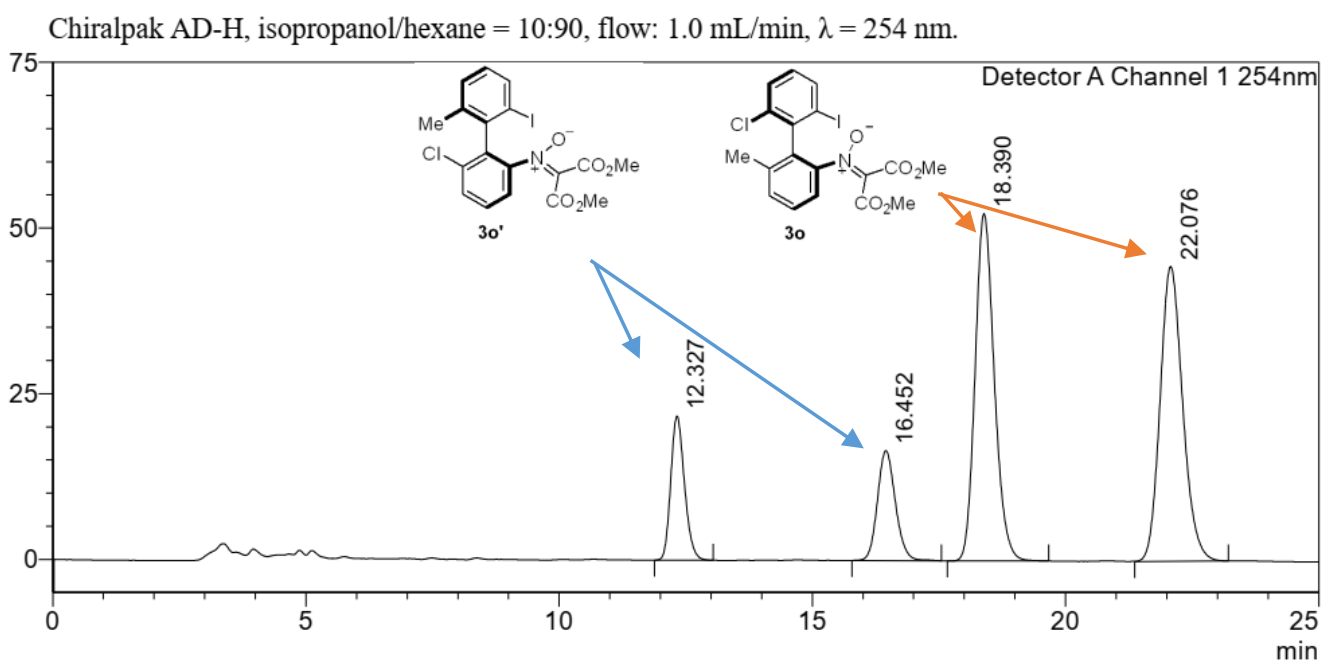

<Peak Table>

Detector A Channel $1254 \mathrm{~nm}$

\begin{tabular}{|r|r|r|r|r|r|r|r|}
\hline Peak\# & Ret. Time & \multicolumn{1}{c|}{ Area } & Height & Conc. & Unit & Mark & Name \\
\hline 1 & 12.327 & 410181 & 21736 & 11.447 & & V & \\
\hline 2 & 16.452 & 410259 & 16603 & 11.449 & & V & \\
\hline 3 & 18.390 & 1381399 & 52416 & 38.551 & & & \\
\hline 4 & 22.076 & 1381489 & 44486 & 38.553 & & V & \\
\hline Total & & 3583328 & 135241 & & & & \\
\hline
\end{tabular}

Figure S91. HPLC spectra of 3o\&30'-rac 


\section{$<$ Chromatogram $>$}

$\mathrm{mV}$

Chiralpak AD-H, isopropanol $/$ hexane $=10: 90$, flow: $1.0 \mathrm{~mL} / \mathrm{min}, \lambda=254 \mathrm{~nm}$.

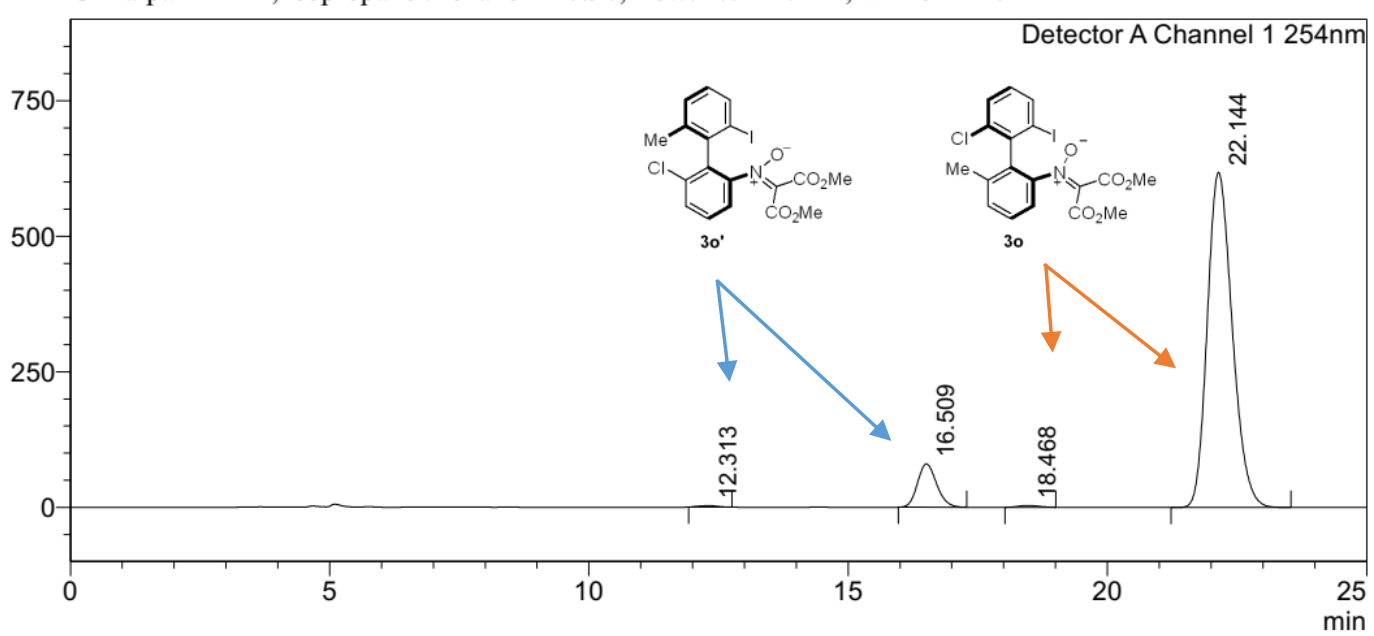

<Peak Table>

Detector A Channel $1254 n m$

\begin{tabular}{|r|r|r|r|r|r|r|r|}
\hline Peak\# & Ret. Time & \multicolumn{1}{c|}{ Area } & \multicolumn{1}{c|}{ Height } & Conc. & Unit & Mark & Name \\
\hline 1 & 12.313 & 67387 & 2752 & 0.295 & & $\mathrm{M}$ & \\
\hline 2 & 16.509 & 2049812 & 79951 & 8.962 & & $\mathrm{M}$ & \\
\hline 3 & 18.468 & 88586 & 3299 & 0.387 & & $\mathrm{M}$ & \\
\hline 4 & 22.144 & 20667097 & 617936 & 90.356 & & $\mathrm{M}$ & \\
\hline Total & & 22872882 & 703939 & & & & \\
\hline
\end{tabular}

Normalization

30'

\begin{tabular}{|r|r|r|r|r|r|r|r|}
\hline Peak\# & Ret. Time & \multicolumn{1}{|c|}{ Area } & \multicolumn{1}{c|}{ Height } & Conc. & Unit & Mark & Name \\
\hline 1 & 12.313 & 67387 & 2752 & 3.183 & & $\mathrm{M}$ & \\
\hline 2 & 16.509 & 2049812 & 79951 & 96.817 & & $\mathrm{M}$ & \\
\hline Total & & 2117199 & 82703 & & & & \\
\hline
\end{tabular}

30

\begin{tabular}{|r|r|r|r|r|r|r|r|}
\hline Peak\# & Ret. Time & \multicolumn{1}{|c|}{ Area } & Height & Conc. & Unit & Mark & Name \\
\hline 3 & 18.468 & 88586 & 3299 & 0.427 & & $\mathrm{M}$ & \\
\hline 4 & 22.144 & 20667097 & 617936 & 99.573 & & $\mathrm{M}$ & \\
\hline Total & & 20755683 & 621236 & & & &
\end{tabular}

Figure S92. HPLC spectra of 3o\&3o' 
<Chromatogram>

$\mathrm{mV}$

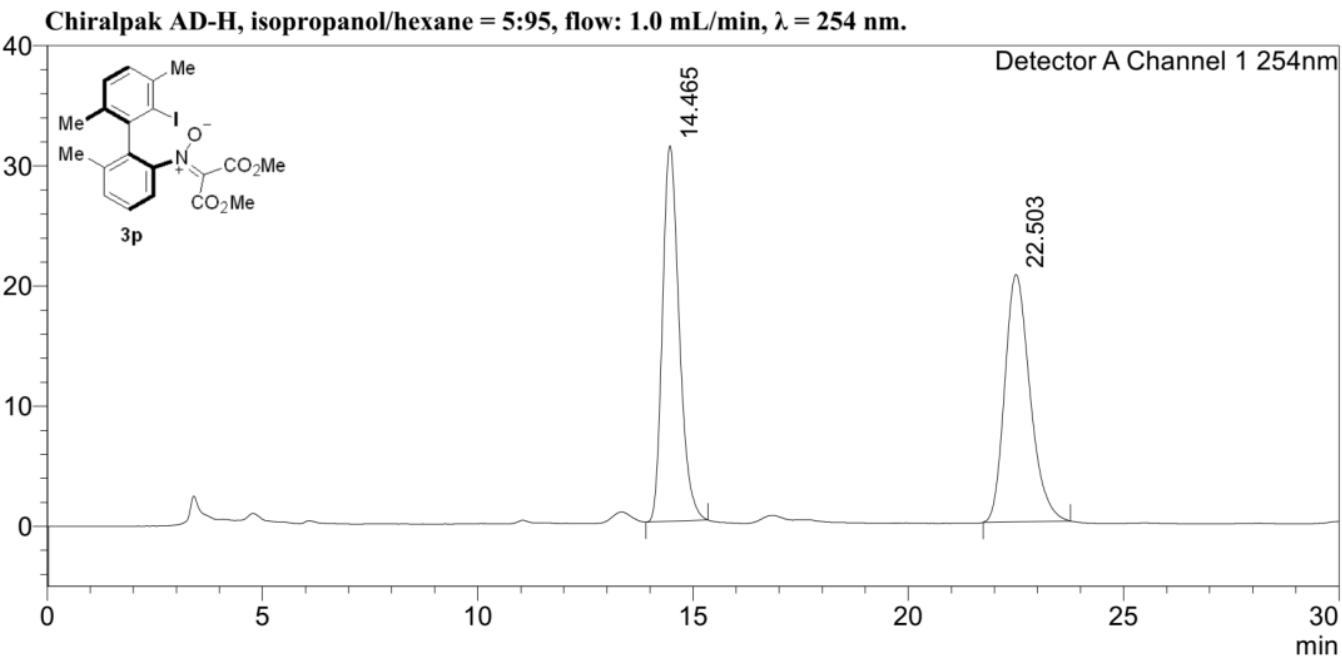

<Peak Table>

Detector A Channel $1254 \mathrm{~nm}$

\begin{tabular}{|r|r|r|r|r|r|r|r|}
\hline Peak\# Ret. Time & \multicolumn{1}{|c|}{ Area } & \multicolumn{1}{|c|}{ Height } & \multicolumn{1}{c|}{ Conc. } & Unit & Mark & Name \\
\hline 1 & 14.465 & 846903 & 31262 & 50.120 & & $\mathrm{M}$ & \\
\hline 2 & 22.503 & 842860 & 20589 & 49.880 & & $\mathrm{M}$ & \\
\hline Total & & 1689763 & 51851 & & & & \\
\hline
\end{tabular}

Figure S93. HPLC spectra of 3p-rac

\section{<Chromatogram>}

$\mathrm{mV}$

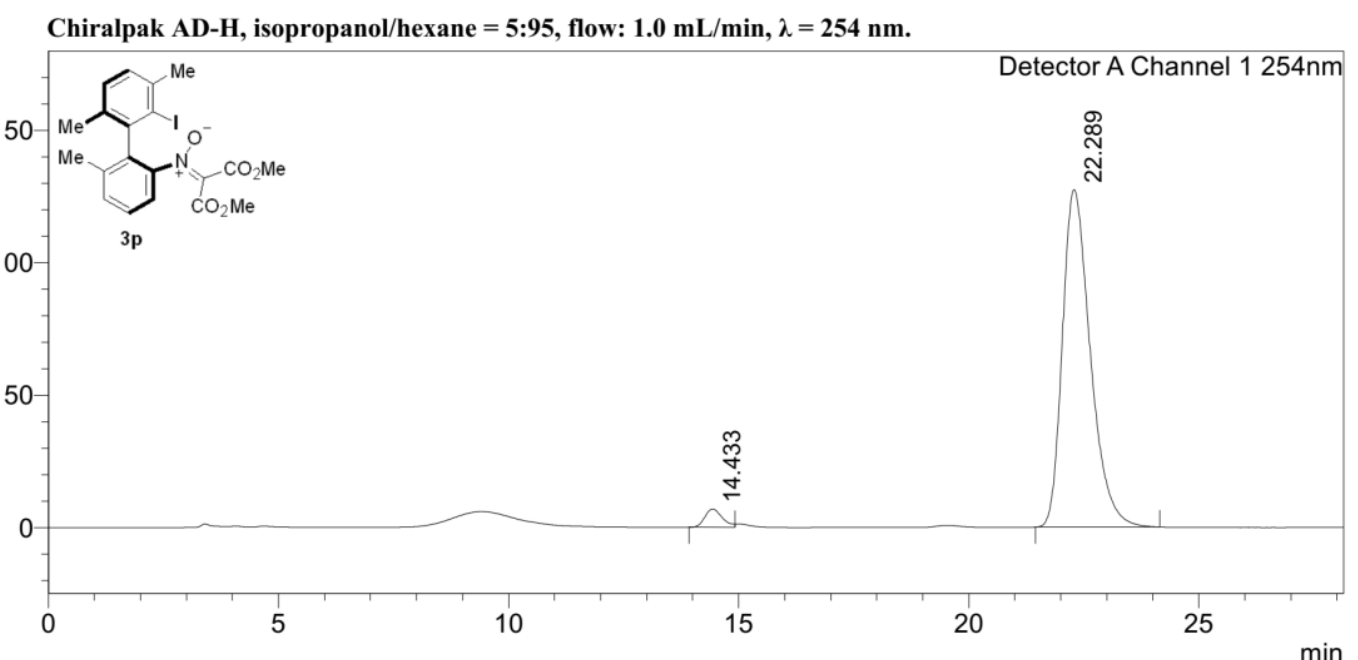

<Peak Table>

Detector A Channel $1254 \mathrm{~nm}$

\begin{tabular}{|r|r|r|r|r|r|r|r|}
\hline Peak\# & Ret. Time & \multicolumn{1}{|c|}{ Area } & \multicolumn{1}{|c|}{ Height } & \multicolumn{1}{c|}{ Conc. } & Unit & Mark & Name \\
\hline 1 & 14.433 & 180373 & 6882 & 3.245 & & $\mathrm{M}$ & \\
\hline 2 & 22.289 & 5378129 & 127484 & 96.755 & & $\mathrm{M}$ & \\
\hline Total & & 5558502 & 134366 & & & & \\
\hline
\end{tabular}

Figure S94. HPLC spectra of 3p 
<Chromatogram>

$\mathrm{mV}$

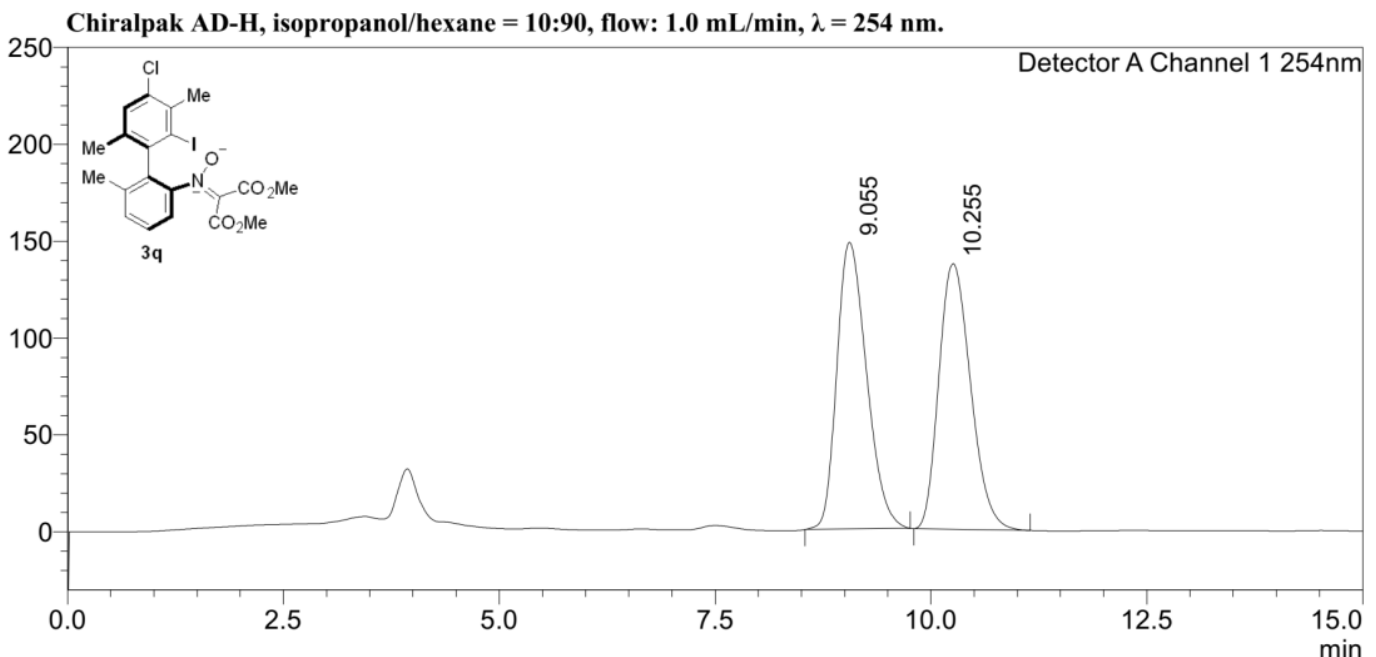

<Peak Table>

Detector A Channel $1254 \mathrm{~nm}$

\begin{tabular}{|r|r|r|r|r|r|r|r|}
\hline Peak\# & Ret. Time & Area & Height & \multicolumn{1}{c|}{ Conc. } & Unit & Mark & Name \\
\hline 1 & 9.055 & 3552504 & 148003 & 50.380 & & $\mathrm{M}$ & \\
\hline 2 & 10.255 & 3498886 & 137139 & 49.620 & & $\mathrm{M}$ & \\
\hline Total & & 7051390 & 285143 & & & & \\
\hline
\end{tabular}

Figure S95. HPLC spectra of 3q-rac

\section{$<$ Chromatogram $>$}

$\mathrm{mV}$

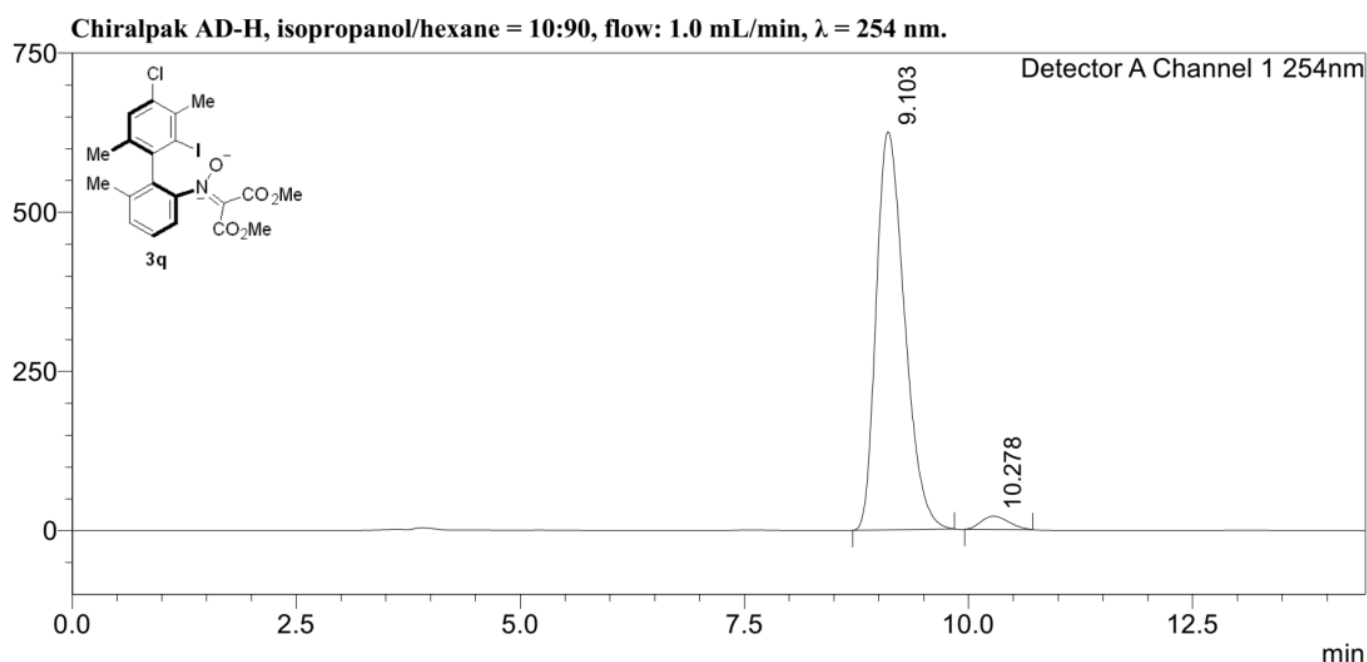

<Peak Table>

Detector A Channel $1254 \mathrm{~nm}$

\begin{tabular}{|r|r|r|r|r|r|r|r|}
\hline Peak\# Ret. Time & \multicolumn{1}{|c|}{ Area } & \multicolumn{1}{c|}{ Height } & \multicolumn{1}{c|}{ Conc. } & Unit & Mark & Name \\
\hline 1 & 9.103 & 13744503 & 624771 & 96.860 & & $\mathrm{M}$ & \\
\hline 2 & 10.278 & 445567 & 20760 & 3.140 & & $\mathrm{M}$ & \\
\hline Total & & 14190070 & 645531 & & & & \\
\hline
\end{tabular}

Figure S96. HPLC spectra of 3q 


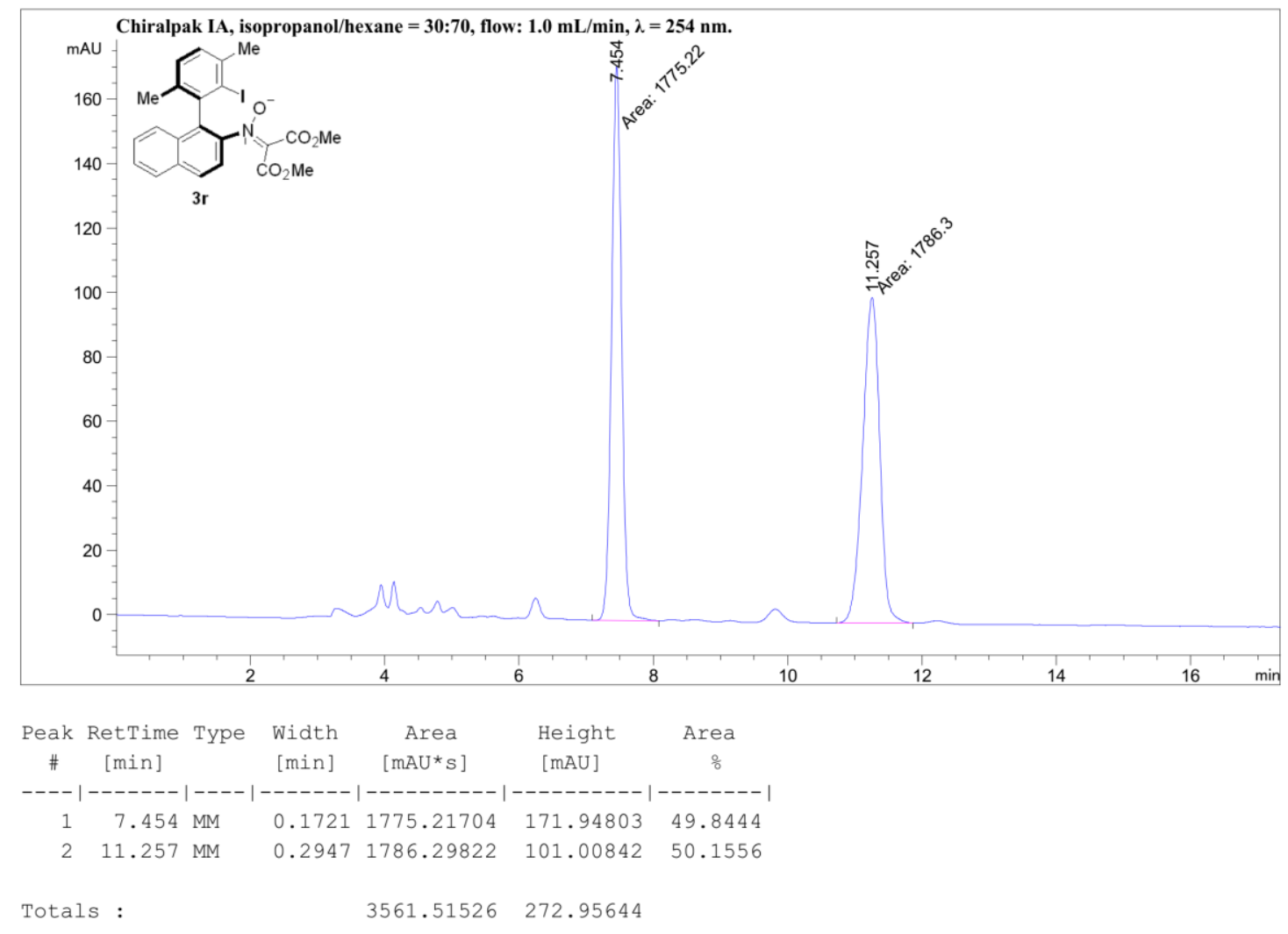

Figure S97. HPLC spectra of 3r-rac

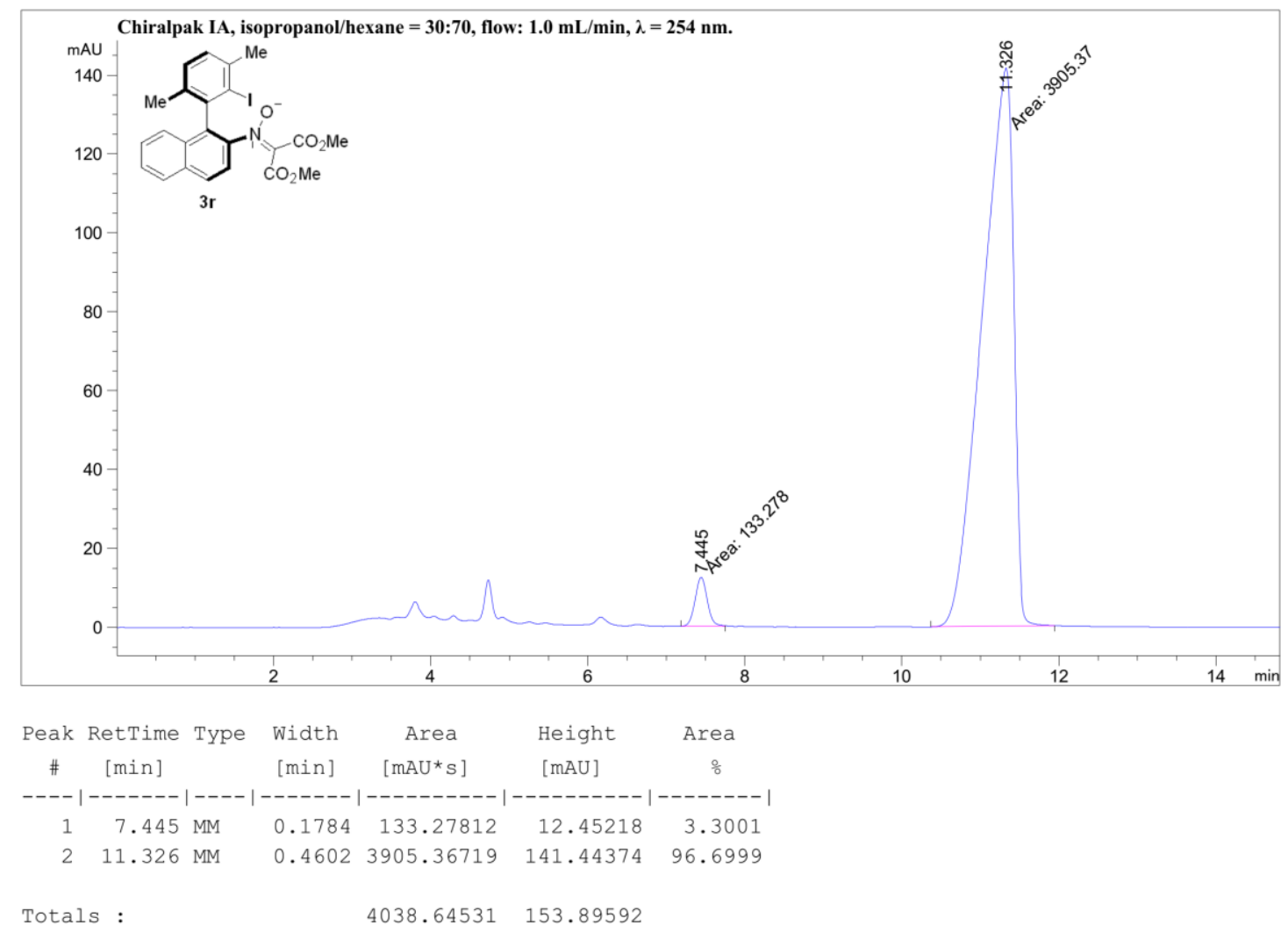

Figure S98. HPLC spectra of $\mathbf{3 r}$ 


\section{$<$ Chromatogram>}

$\mathrm{mV}$ Chiralpak AD-H, isopropanol/hexane $=5: 95$, flow: $1.0 \mathrm{~mL} / \mathrm{min}, \lambda=254 \mathrm{~nm}$.

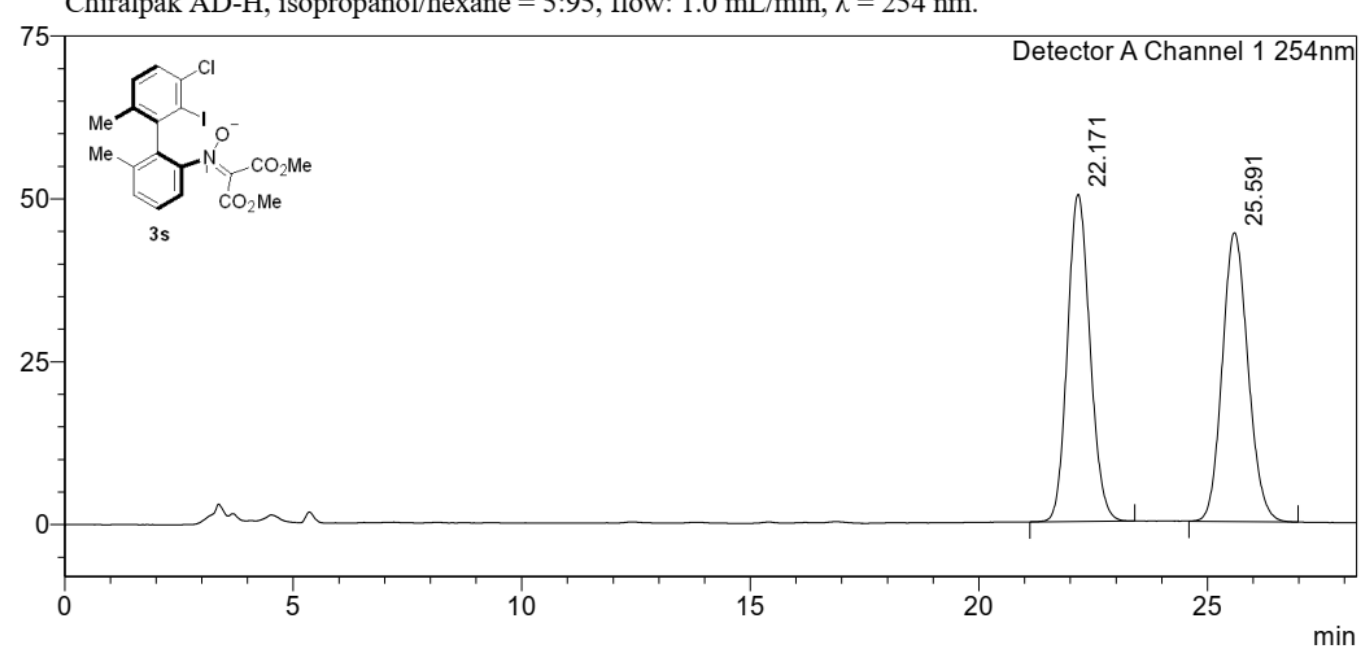

<Peak Table>

Detector A Channel $1254 \mathrm{~nm}$

\begin{tabular}{|r|r|r|r|r|r|r|r|}
\hline Peak\# & Ret. Time & Area & Height & Conc. & Unit & Mark & Name \\
\hline 1 & 22.171 & 1722332 & 50230 & 49.760 & & SV & \\
\hline 2 & 25.591 & 1738966 & 44359 & 50.240 & & V & \\
\hline Total & & 3461299 & 94589 & & & &
\end{tabular}

Figure S99. HPLC spectra of 3s-rac

\section{<Chromatogram>}

$\mathrm{mV}$ Chiralpak AD-H, isopropanol/hexane $=5: 95$, flow: $1.0 \mathrm{~mL} / \mathrm{min}, \lambda=254 \mathrm{~nm}$.

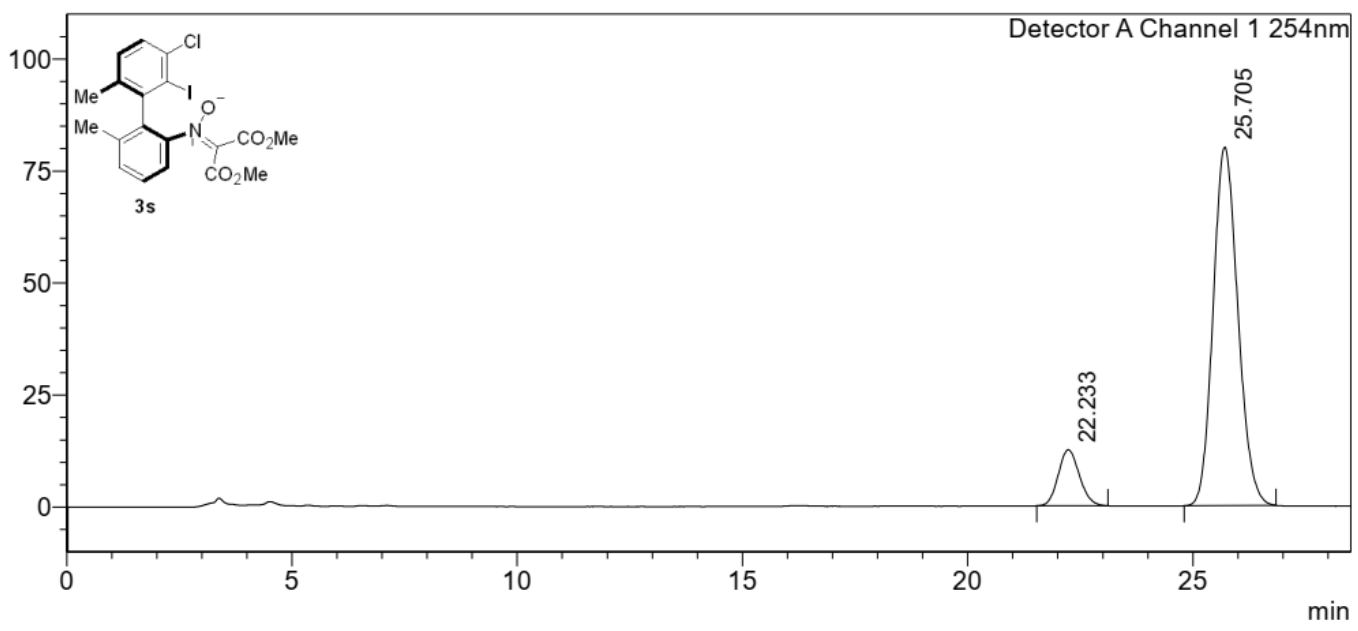

<Peak Table>

Detector A Channel $1254 \mathrm{~nm}$ Peak\# Ret. Time

\begin{tabular}{|r|r|r|r|r|r|r|r|}
\hline 1 & 22.233 & 407944 & 12512 & 11.768 & & $\mathrm{M}$ & \\
\hline 2 & 25.705 & 3058612 & 79982 & 88.232 & & $\mathrm{M}$ & \\
\hline Total & & 3466556 & 92494 & & & & \\
\hline
\end{tabular}

Figure S100. HPLC spectra of $\mathbf{3 s}$ 


\section{$<$ Chromatogram $>$}

$\mathrm{mV} \quad$ Chiralpak AD-H, isopropanol/hexane $=30: 70$, flow: $1.0 \mathrm{~mL} / \mathrm{min}, \lambda=254 \mathrm{~nm}$.

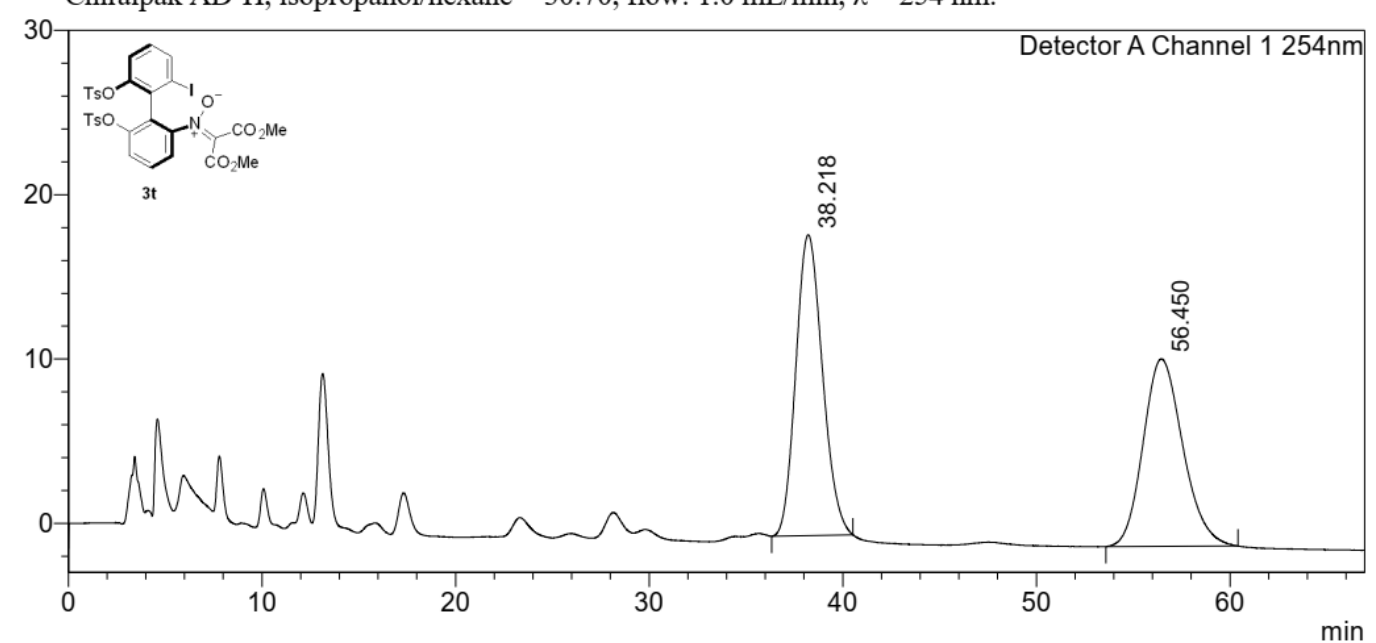

<Peak Table>

Detector A Channel $1254 \mathrm{~nm}$

\begin{tabular}{|r|r|r|r|r|r|r|r|}
\hline Peak\# & Ret. Time & Area & Height & Conc. & Unit & Mark & Name \\
\hline 1 & 38.218 & 1752127 & 18316 & 51.869 & & $\mathrm{M}$ & \\
\hline 2 & 56.450 & 1625831 & 11414 & 48.131 & & $\mathrm{M}$ & \\
\hline Total & & 3377958 & 29731 & & & & \\
\hline
\end{tabular}

Figure S101. HPLC spectra of 3t-rac

\section{$<$ Chromatogram $>$}

$\mathrm{mV}$

Chiralpak AD-H, isopropanol $/$ hexane $=30: 70$, flow: $1.0 \mathrm{~mL} / \mathrm{min}, \lambda=254 \mathrm{~nm}$

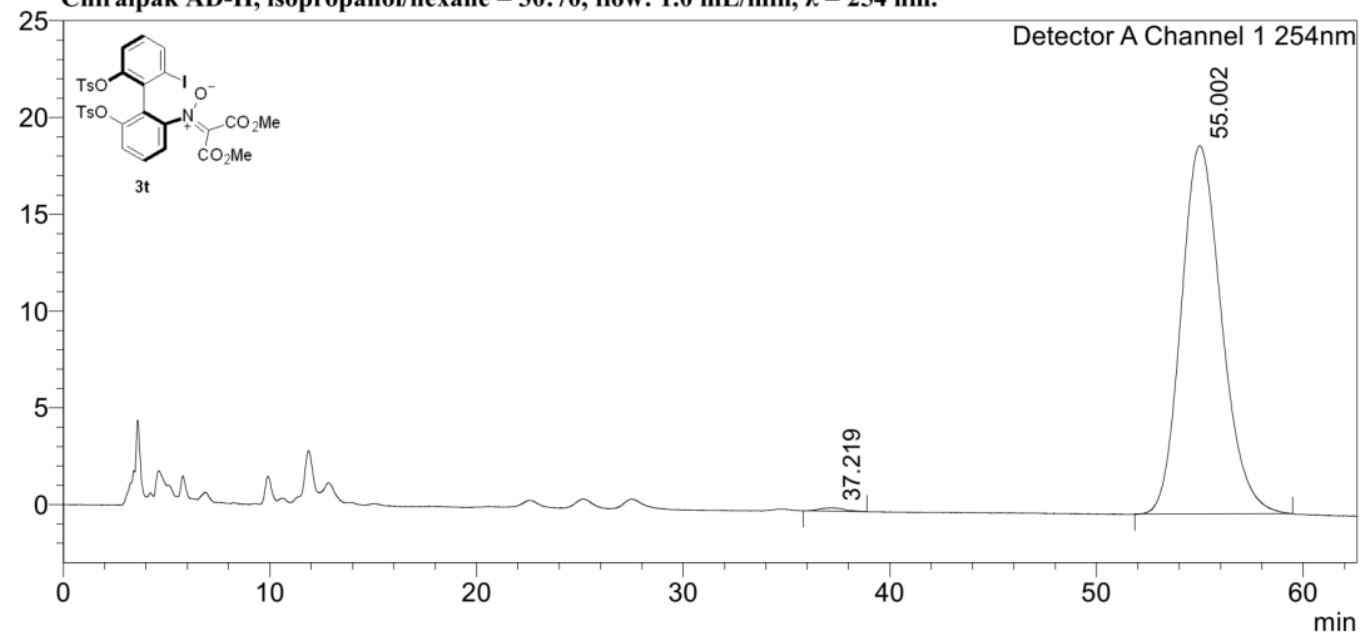

$<$ Peak Table>

Detector A Channel $1254 \mathrm{~nm}$

\begin{tabular}{|r|r|r|r|r|r|r|r|}
\hline Peak\# & Ret. Time & \multicolumn{1}{|c|}{ Area } & \multicolumn{1}{|c|}{ Height } & \multicolumn{1}{c|}{ Conc. } & Unit & Mark & Name \\
\hline 1 & 37.219 & 14053 & 175 & 0.539 & & $\mathrm{M}$ & \\
\hline 2 & 55.002 & 2591484 & 19022 & 99.461 & & $\mathrm{M}$ & \\
\hline Total & & 2605537 & 19196 & & & & \\
\hline
\end{tabular}

Figure S102. HPLC spectra of $\mathbf{3 t}$ 
<Chromatogram>

$\mathrm{mV}$

Chiralpak AD-H, isopropanol/hexane $=2.5: 97.5$, flow: $1.0 \mathrm{~mL} / \mathrm{min}, \lambda=254 \mathrm{~nm}$.

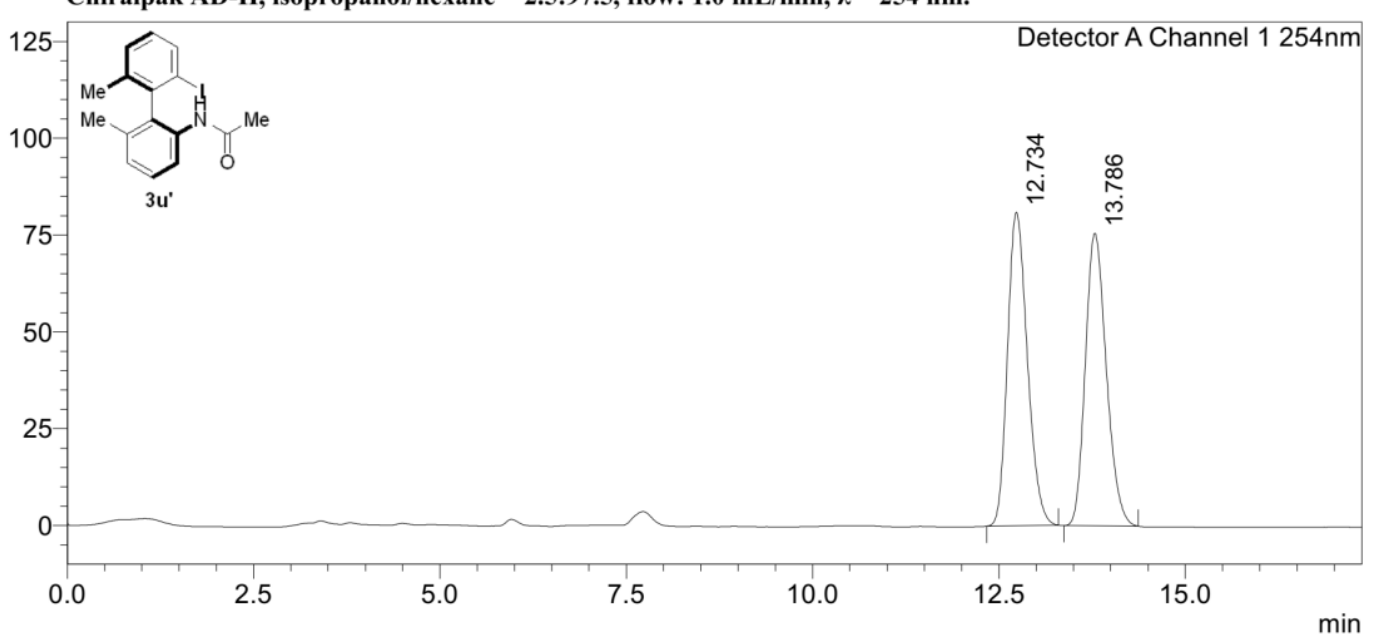

<Peak Table>

Detector A Channel 1 254nm

\begin{tabular}{|r|r|r|r|r|r|r|r|}
\hline Peak\# Ret. Time & \multicolumn{1}{|c|}{ Area } & \multicolumn{1}{|c|}{ Height } & \multicolumn{1}{c|}{ Conc. } & Unit & Mark & Name \\
\hline 1 & 12.734 & 1501422 & 80938 & 50.348 & & $\mathrm{M}$ & \\
\hline 2 & 13.786 & 1480691 & 75575 & 49.652 & & $\mathrm{M}$ & \\
\hline Total & & 2982114 & 156513 & & & & \\
\hline
\end{tabular}

Figure S103. HPLC spectra of 3u'-rac

\section{$<$ Chromatogram $>$}

$\mathrm{mV}$ Chiralpak AD-H, isopropanol $/$ hexane $=2.5: 97.5$, flow: $1.0 \mathrm{~mL} / \mathrm{min}, \lambda=254 \mathrm{~nm}$.

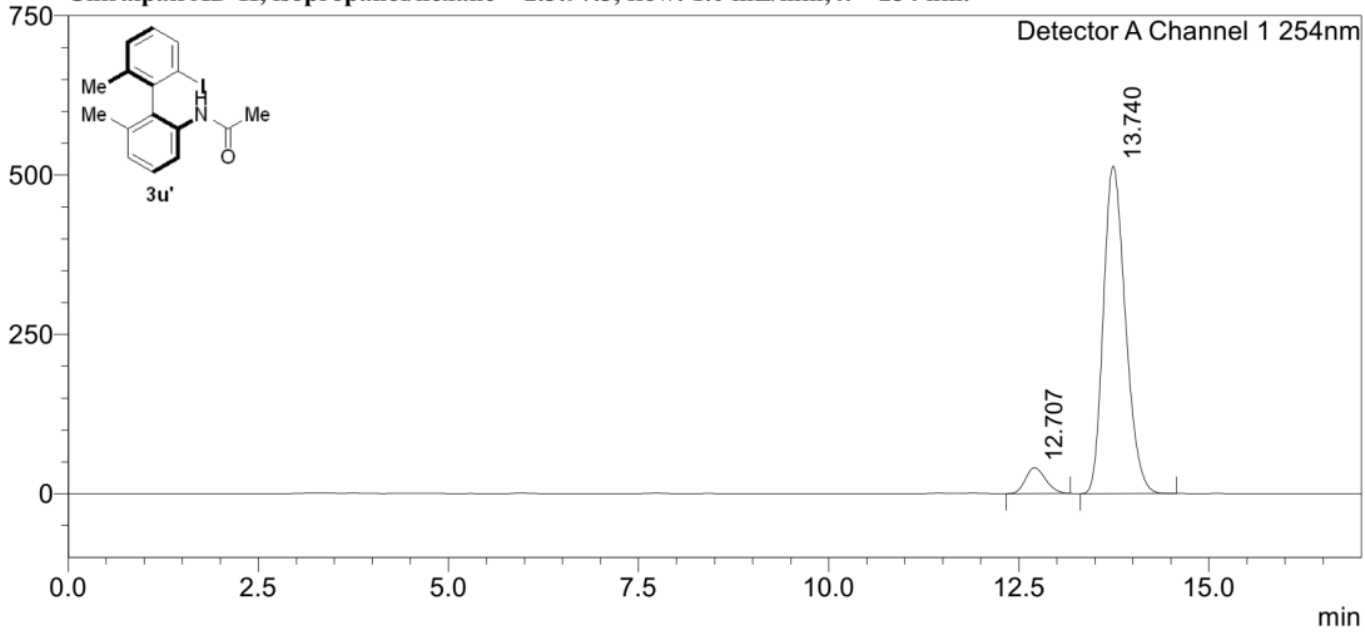

<Peak Table>

Detector A Channel $1254 \mathrm{~nm}$

\begin{tabular}{|r|r|r|r|r|r|r|r|}
\hline Peak\# & Ret. Time & \multicolumn{1}{|c|}{ Area } & \multicolumn{1}{|c|}{ Height } & \multicolumn{1}{c|}{ Conc. } & Unit & Mark & Name \\
\hline 1 & 12.707 & 753482 & 40678 & 6.773 & & $\mathrm{M}$ & \\
\hline 2 & 13.740 & 10371472 & 514065 & 93.227 & & $\mathrm{M}$ & \\
\hline Total & & 11124954 & 554743 & & & & \\
\hline
\end{tabular}

Figure S104. HPLC spectra of 3u' 


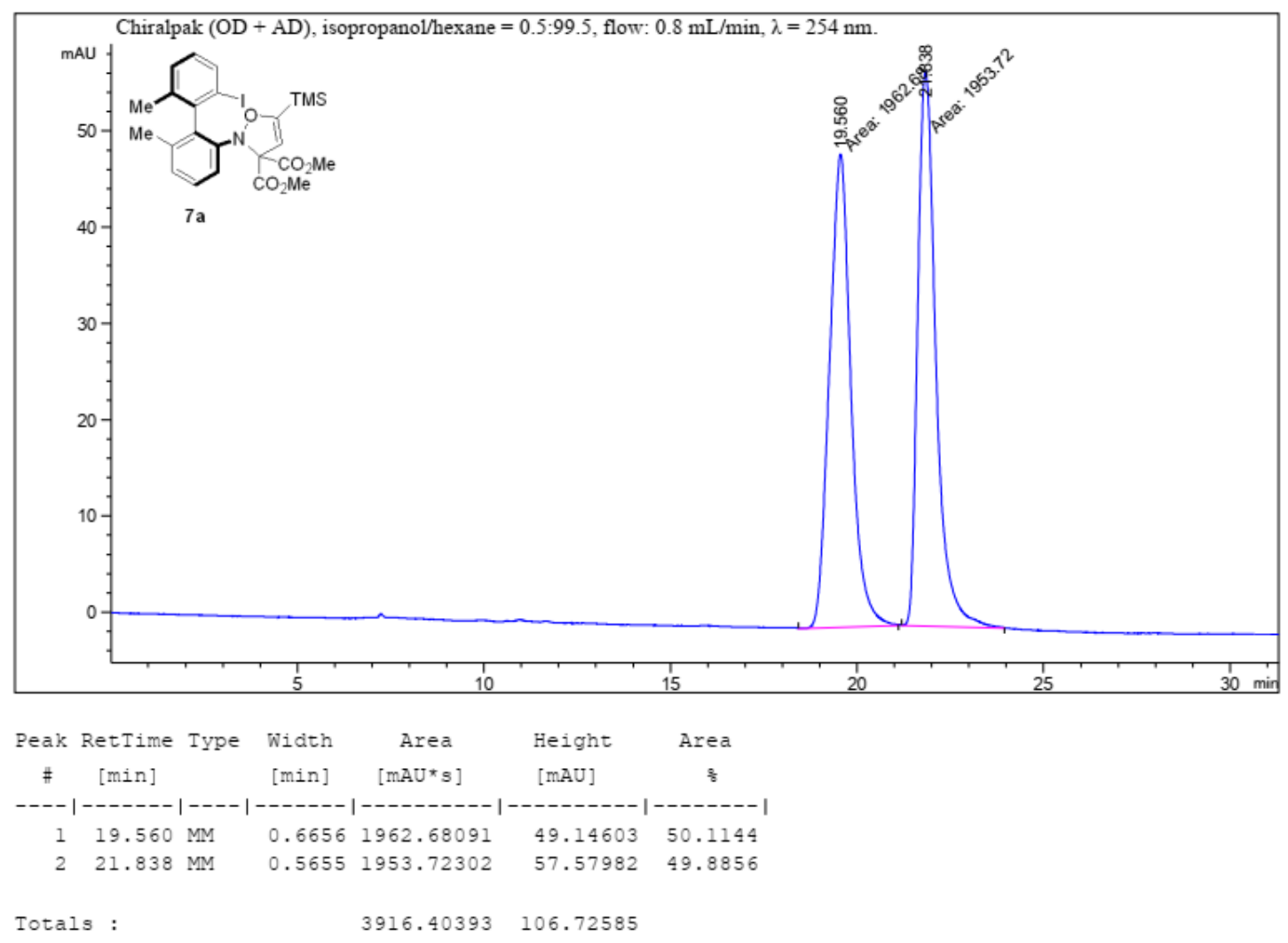

Figure S105. HPLC spectra of 7a-rac 


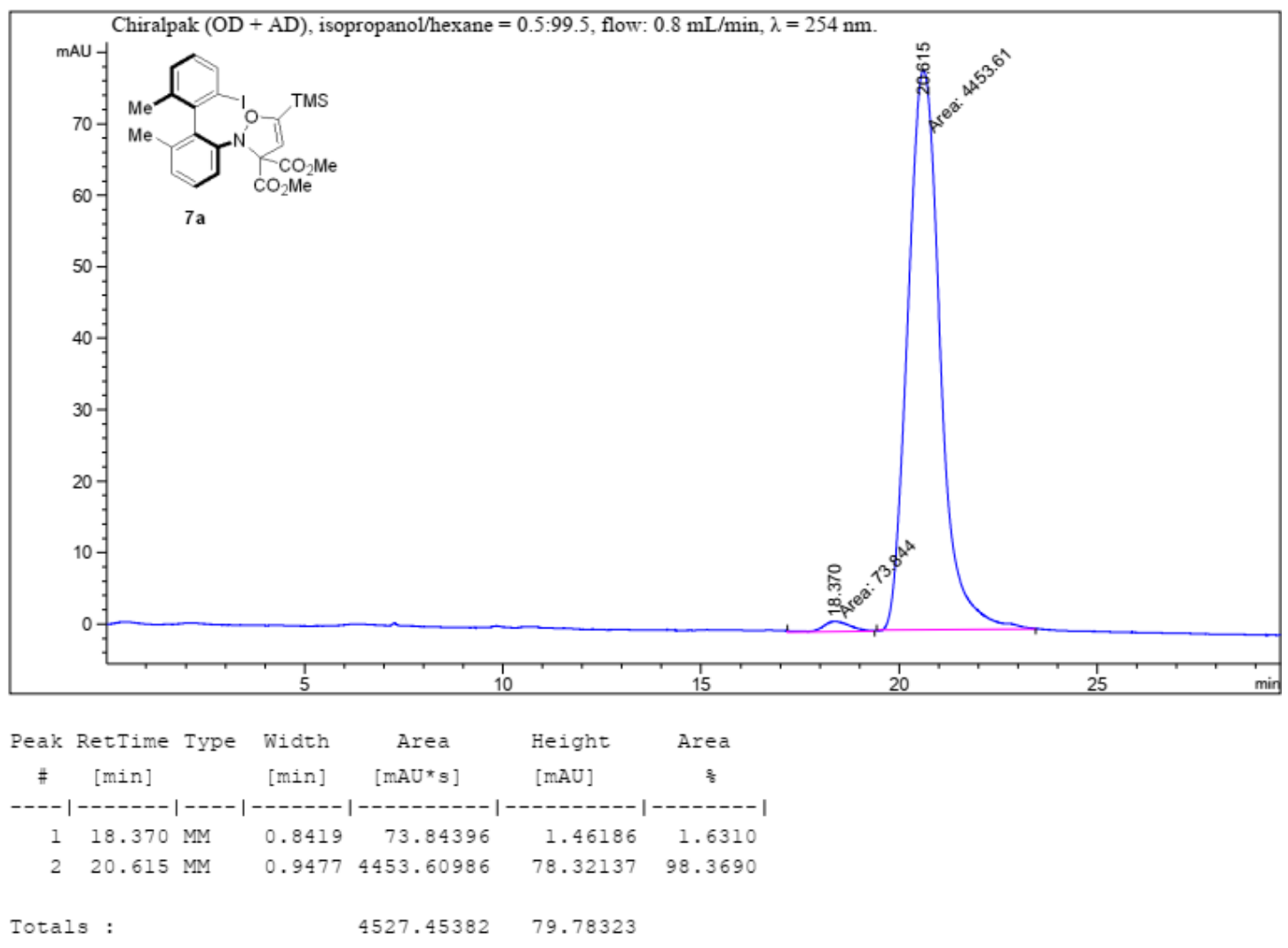

Figure S106. HPLC spectra of 7a

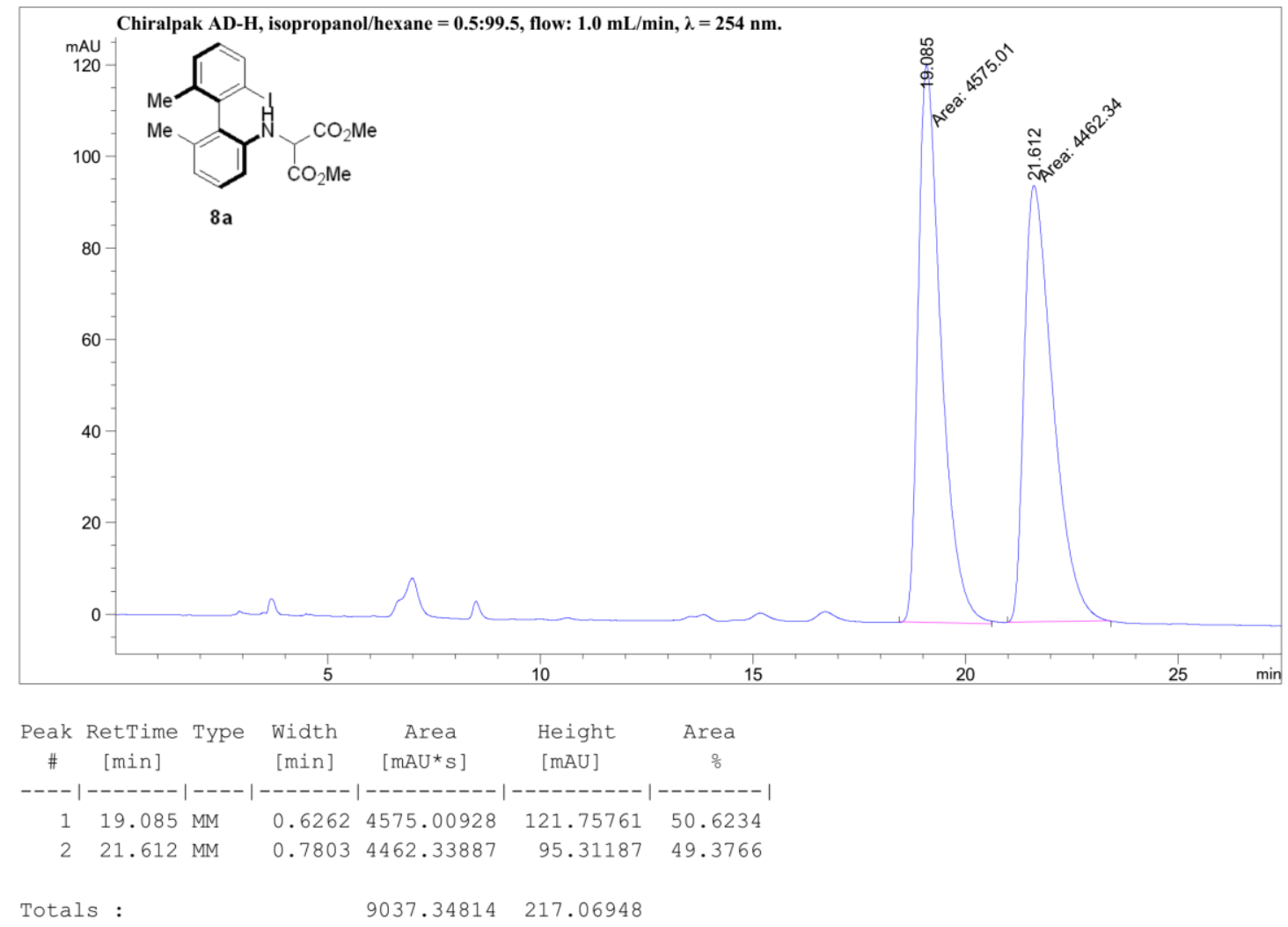

Figure S107. HPLC spectra of 8a-rac 


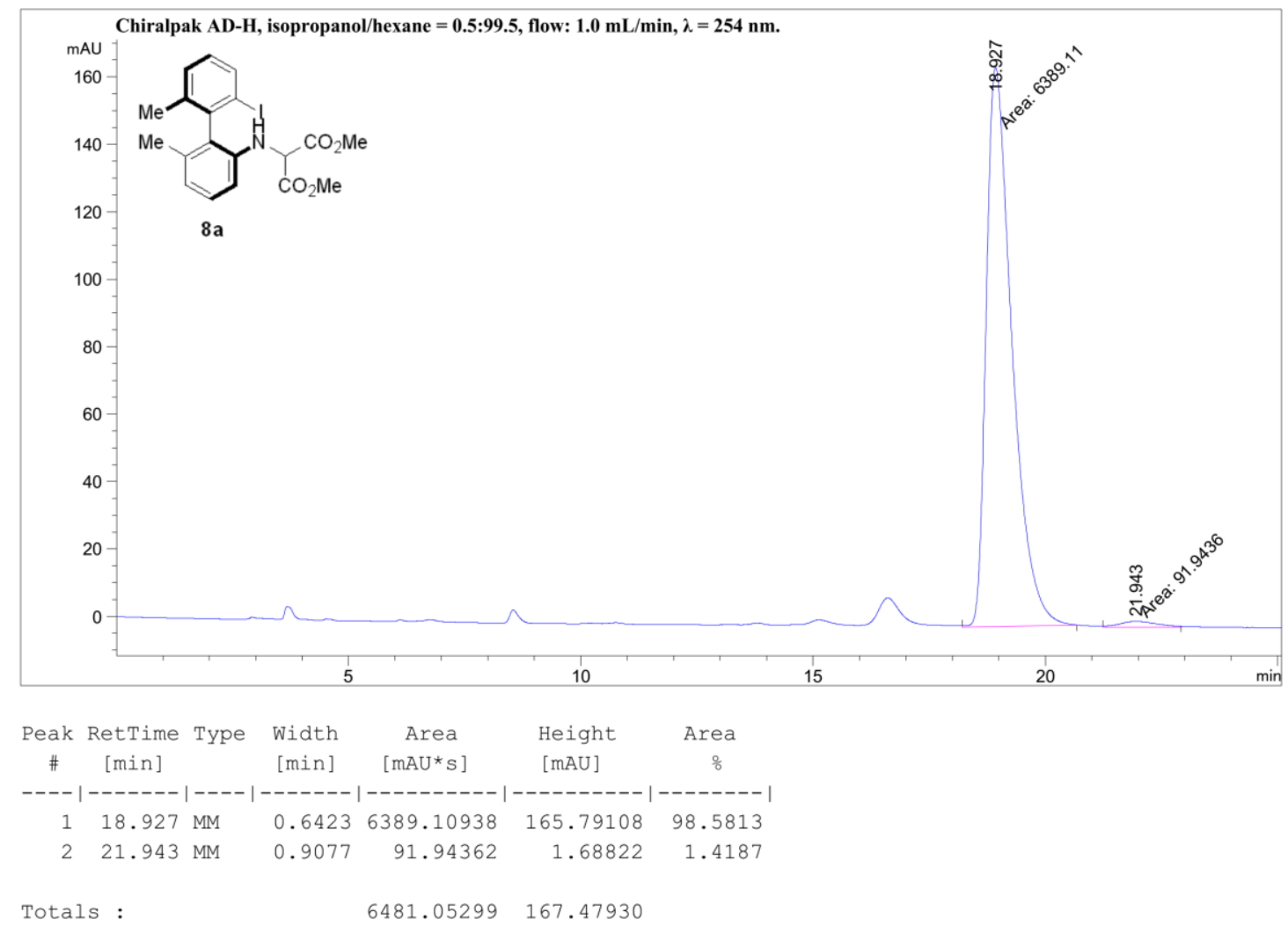

Figure S108. HPLC spectra of $\mathbf{8 a}$

\section{$<$ Chromatogram>}

$\mathrm{mV}$

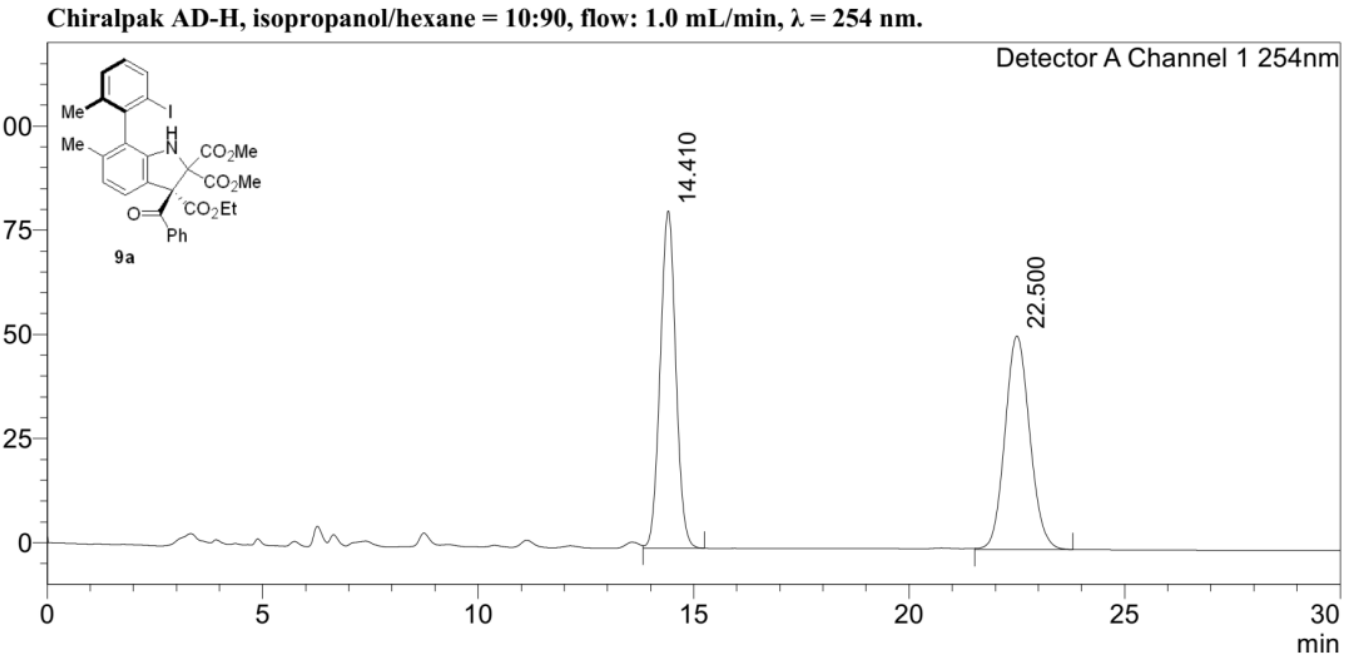

<Peak Table>

Detector A Channel $1254 \mathrm{~nm}$

\begin{tabular}{|r|r|r|r|r|r|r|r|}
\hline Peak\# Ret. Time & \multicolumn{1}{|c|}{ Area } & Height & Conc. & Unit & Mark & Name \\
\hline 1 & 14.410 & 2052843 & 80981 & 49.848 & & $\mathrm{M}$ & \\
\hline Total & 22.500 & 2065327 & 51288 & 50.152 & & $\mathrm{M}$ & \\
\hline
\end{tabular}

Figure S109. HPLC spectra of 9a-rac 
<Chromatogram>

$\mathrm{mV}$ Chiralpak AD-H, isopropanol $/$ hexane $=10: 90$, flow: $1.0 \mathrm{~mL} / \mathrm{min}, \lambda=254 \mathrm{~nm}$.

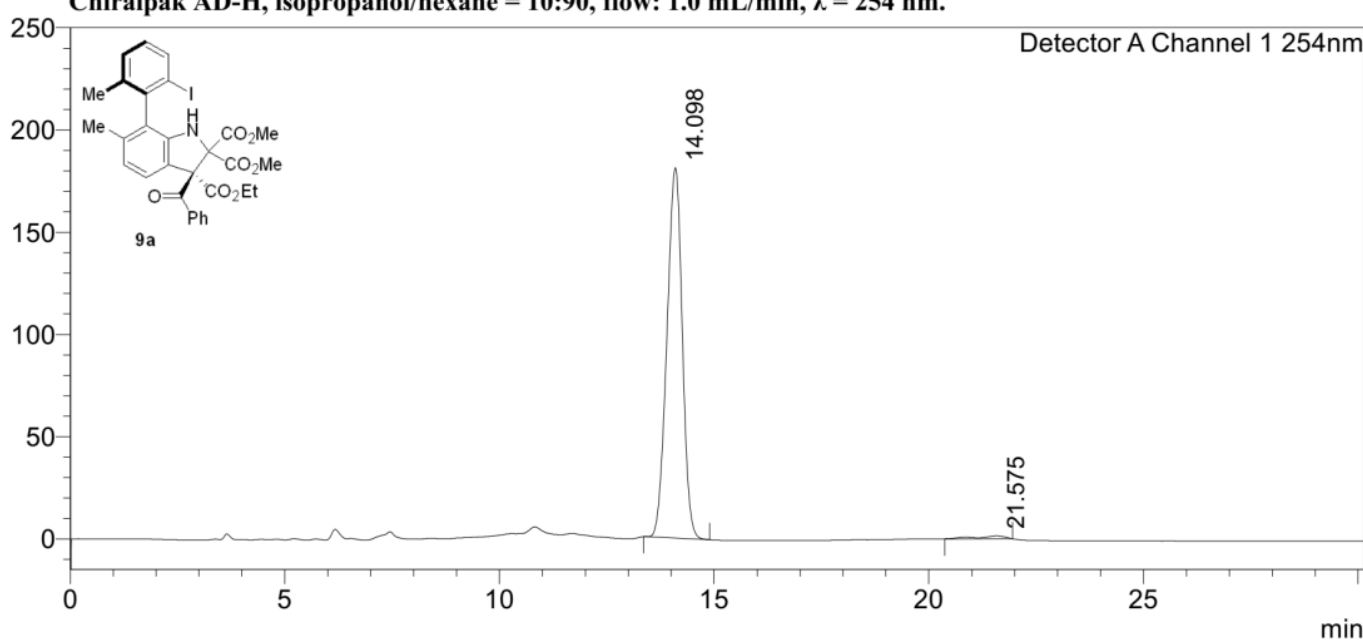

<Peak Table>

Detector A Channel $1254 \mathrm{~nm}$

\begin{tabular}{|r|r|r|r|r|r|r|r|}
\hline Peak\# & Ret. Time & \multicolumn{1}{|c|}{ Area } & Height & \multicolumn{1}{c|}{ Conc. } & Unit & Mark & Name \\
\hline 1 & 14.098 & 4516565 & 181172 & 98.591 & & M & \\
\hline 2 & 21.575 & 64527 & 1414 & 1.409 & & M & \\
\hline Total & & 4581092 & 182587 & & & & \\
\hline
\end{tabular}

Figure S110. HPLC spectra of 9a

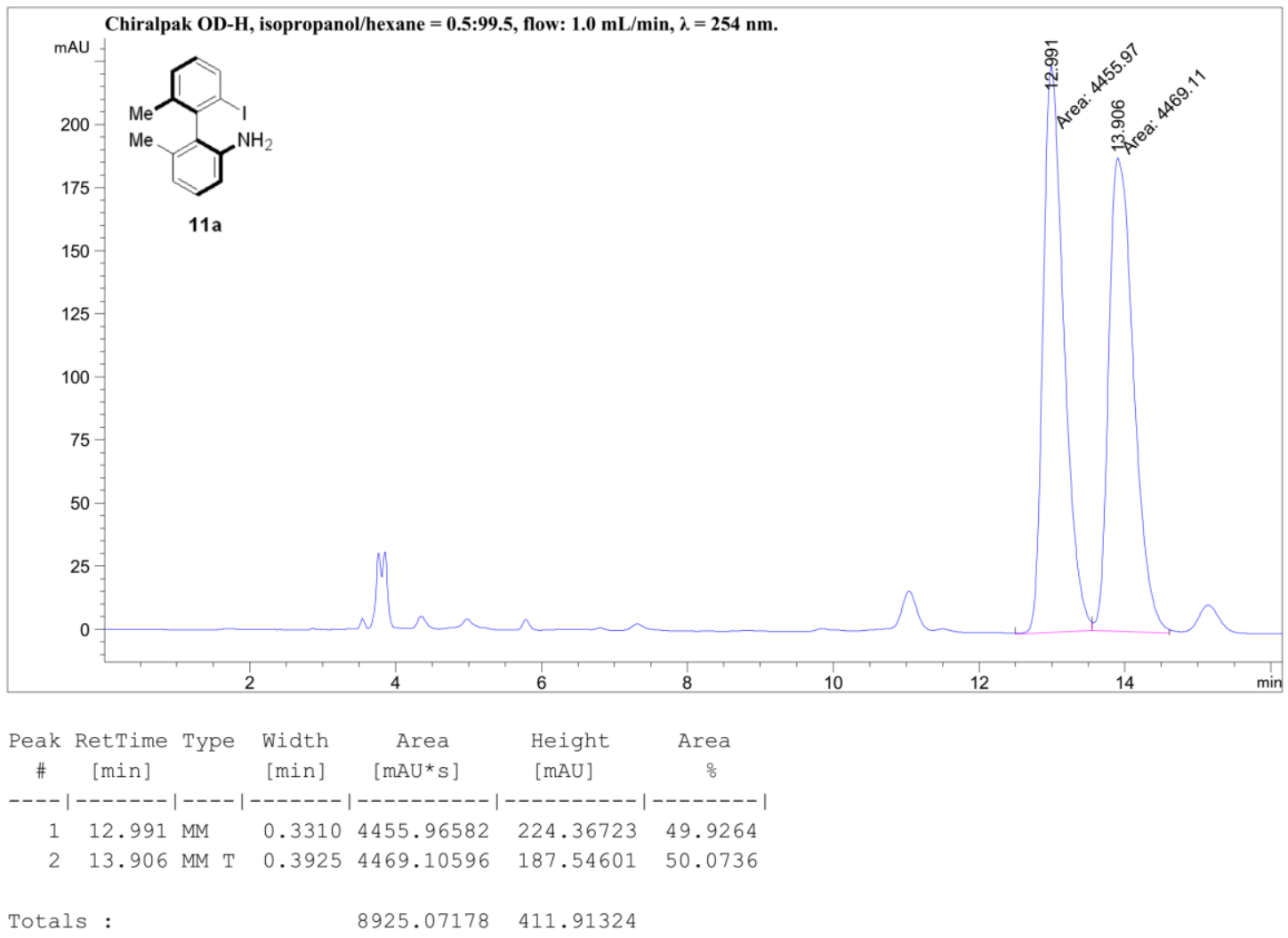

Figure S111. HPLC spectra of 11a-rac 


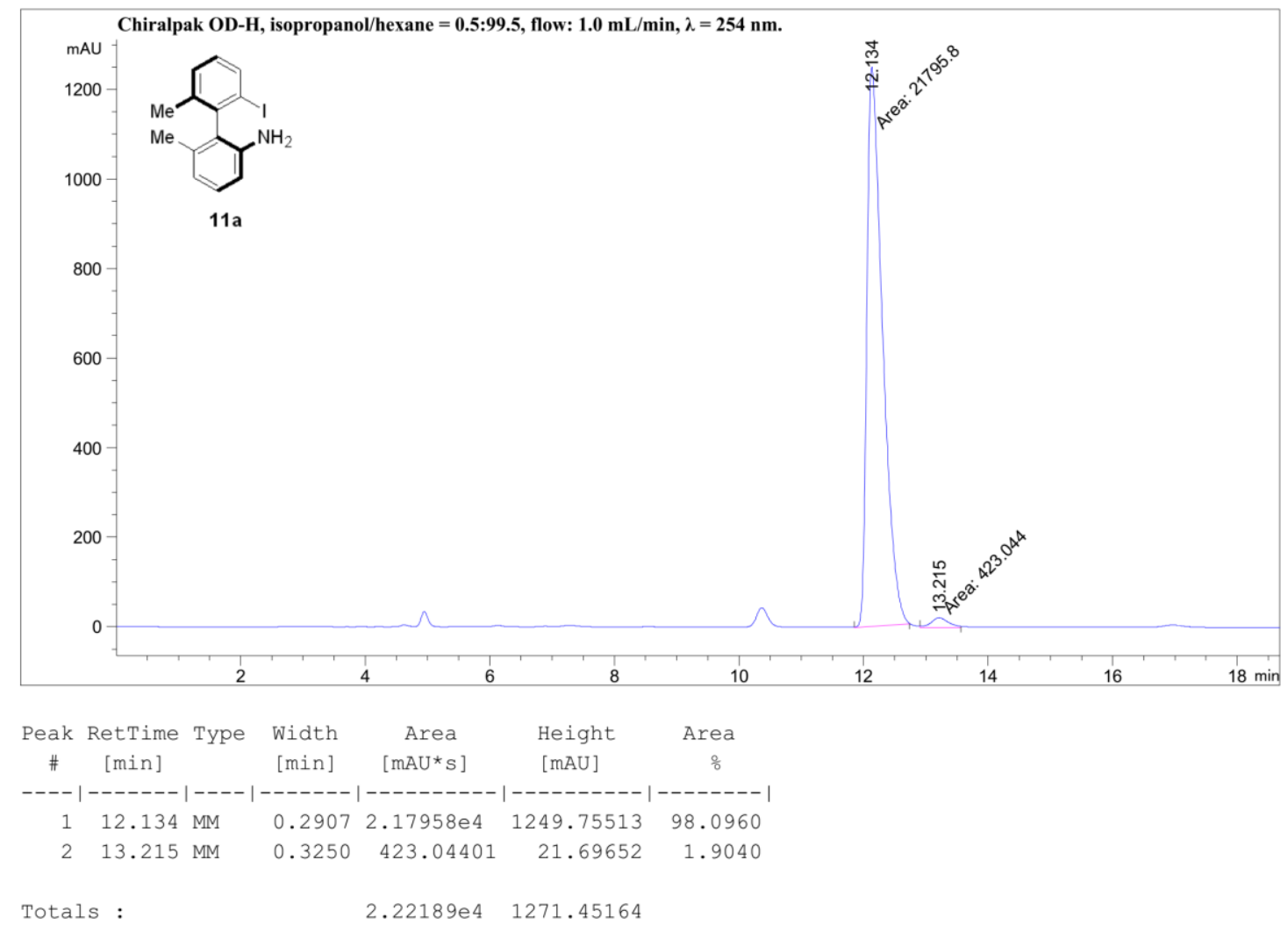

Figure S112. HPLC spectra of 11a

\section{$<$ Chromatogram $>$}

$\mathrm{mV}$

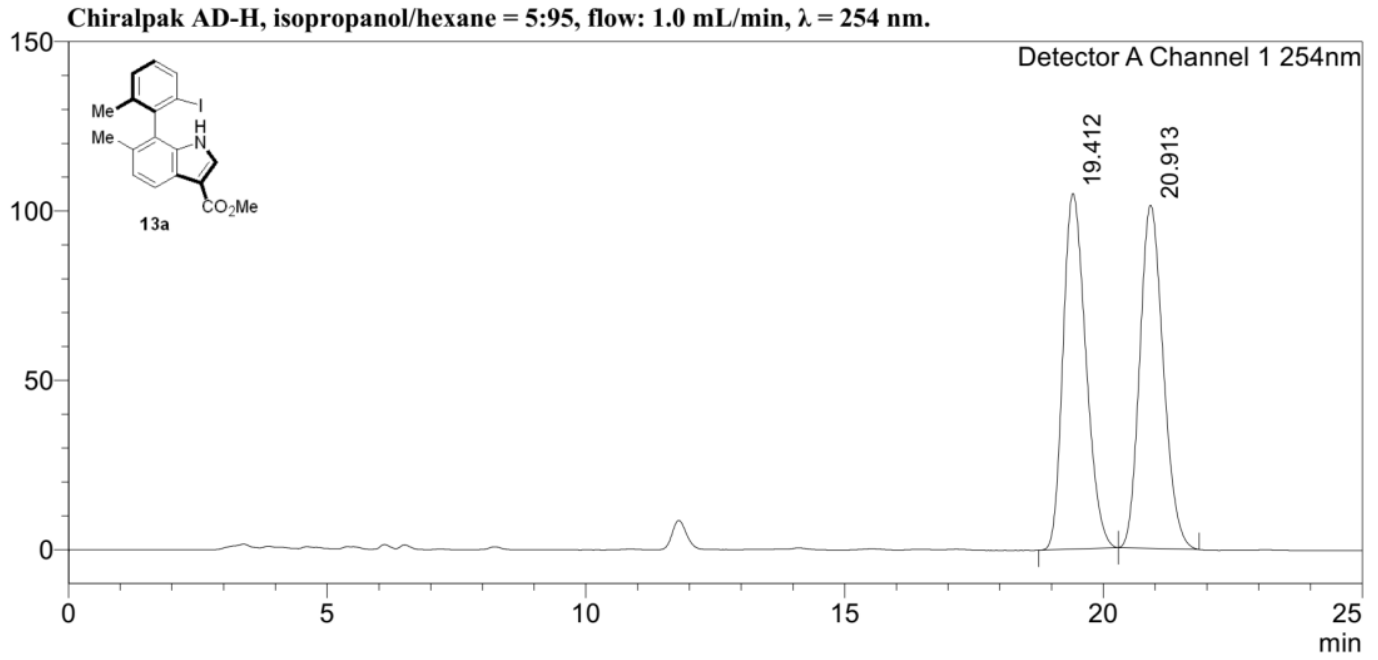

<Peak Table>

Detector A Channel $1254 \mathrm{~nm}$

\begin{tabular}{|r|r|r|r|r|r|r|r|}
\hline Peak\# & Ret. Time & Area & Height & Conc. & Unit & Mark & Name \\
\hline 1 & 19.412 & 3216660 & 105045 & 50.106 & & $\mathrm{M}$ & \\
\hline 2 & 20.913 & 3203002 & 101350 & 49.894 & & $\mathrm{M}$ & \\
\hline Total & & 6419662 & 206395 & & & & \\
\hline
\end{tabular}

Figure S113 HPLC spectra of 13a-rac 
$<$ Chromatogram $>$

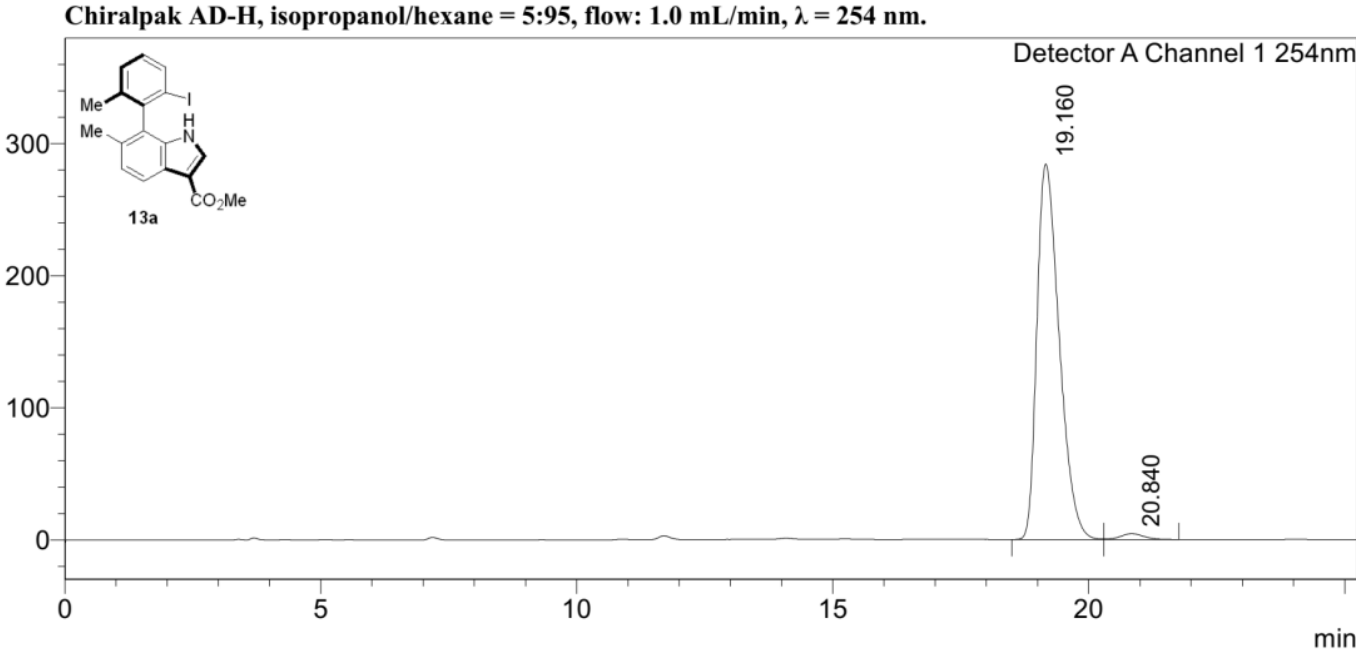

<Peak Table>

Detector A Channel $1254 \mathrm{~nm}$

\begin{tabular}{|r|r|r|r|r|r|r|r|}
\hline Peak\# Ret. Time & \multicolumn{1}{|c|}{ Area } & Height & \multicolumn{1}{|c|}{ Conc. } & Unit & Mark & Name \\
\hline 1 & 19.160 & 8616292 & 284312 & 98.411 & & & \\
\hline 2 & 20.840 & 139089 & 4427 & 1.589 & & $\mathrm{~V}$ & \\
\hline Total & & 8755381 & 288739 & & & & \\
\hline
\end{tabular}

Figure S114. HPLC spectra of 13a

\section{$<$ Chromatogram>}

$\mathrm{mV}$ Chiralpak IC, isopropanol/hexane $=5: 95$, flow: $1.0 \mathrm{~mL} / \mathrm{min}, \lambda=254 \mathrm{~nm}$.

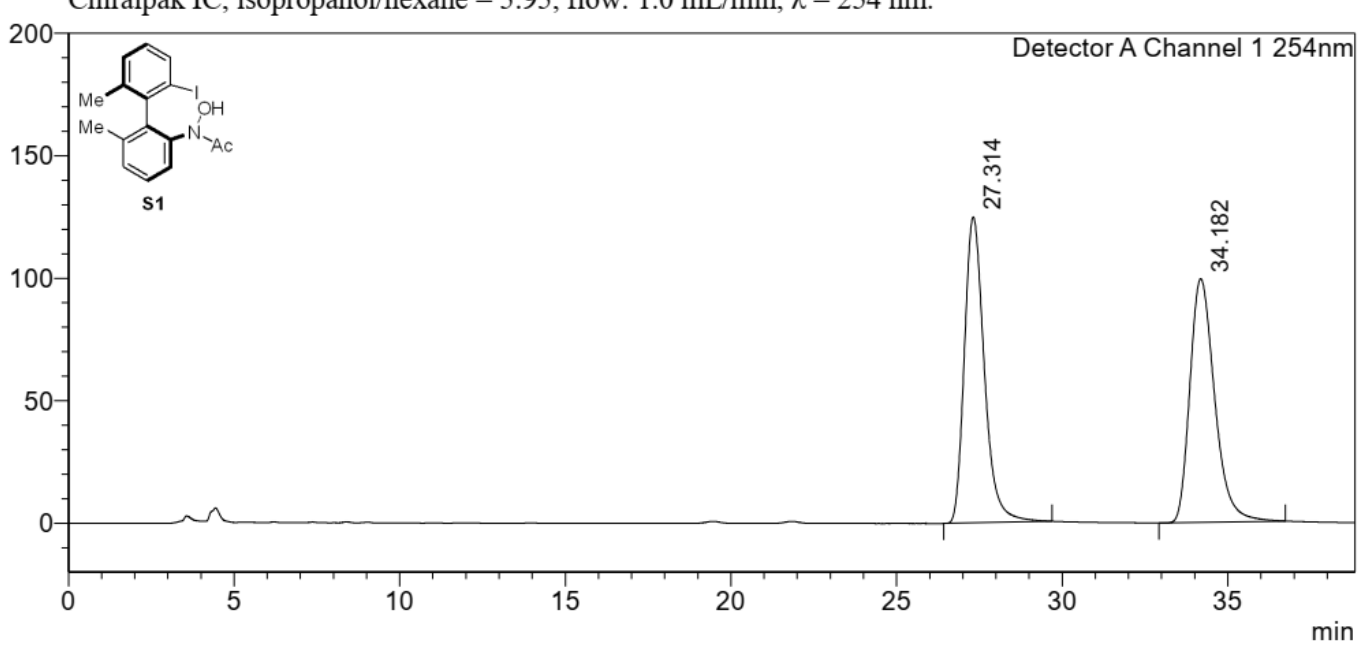

<Peak Table>

Detector A Channel $1254 \mathrm{~nm}$

\begin{tabular}{|r|r|r|r|r|r|r|r|}
\hline Peak\# & Ret. Time & \multicolumn{1}{|c|}{ Area } & \multicolumn{1}{c|}{ Height } & \multicolumn{1}{c|}{ Conc. } & Unit & Mark & Name \\
\hline 1 & 27.314 & 5221788 & 124936 & 50.287 & & $\mathrm{M}$ & \\
\hline 2 & 34.182 & 5162092 & 99489 & 49.713 & & $\mathrm{M}$ & \\
\hline Total & & 10383880 & 224425 & & & & \\
\hline
\end{tabular}

Figure S115. HPLC spectra of S1-rac 


\section{<Chromatogram>}

$\mathrm{mV}$ Chiralpak IC, isopropanol/hexane $=5: 95$, flow: $1.0 \mathrm{~mL} / \mathrm{min}, \lambda=254 \mathrm{~nm}$

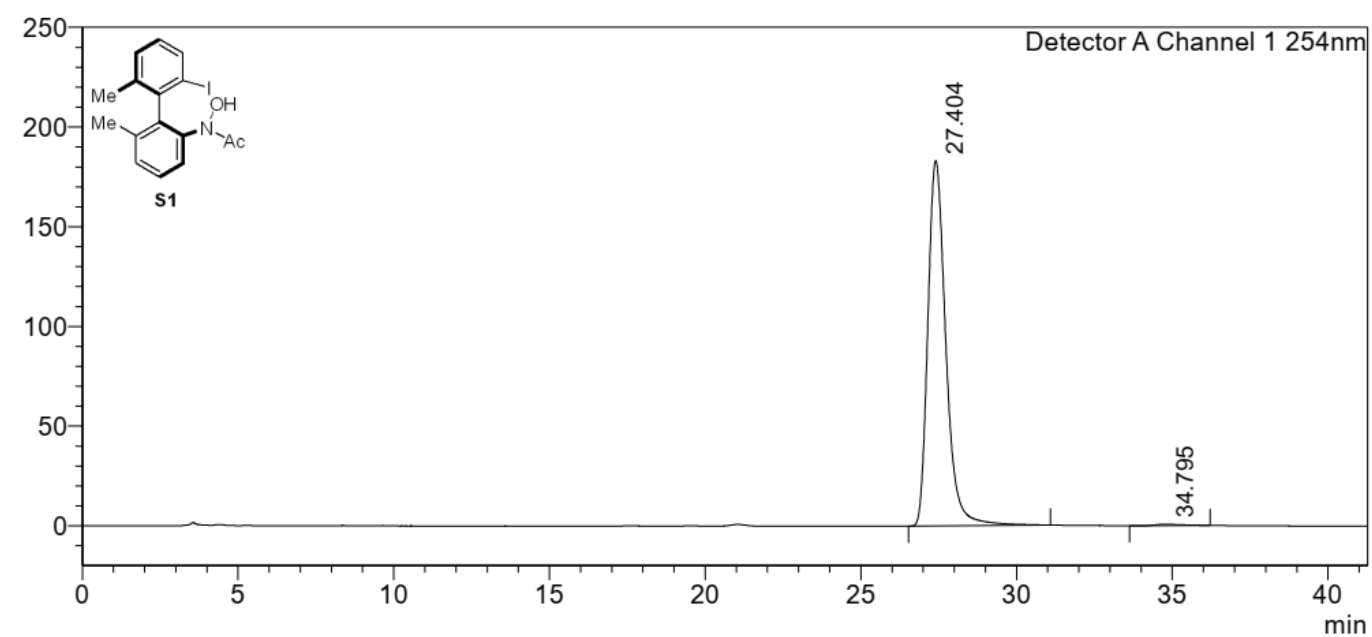

<Peak Table>

Detector A Channel $1254 \mathrm{~nm}$

\begin{tabular}{|r|r|r|r|r|r|r|r|}
\hline Peak\# & Ret. Time & \multicolumn{1}{|c|}{ Area } & Height & \multicolumn{1}{c|}{ Conc. } & Unit & Mark & Name \\
\hline 1 & 27.404 & 7354707 & 183211 & 99.472 & & $\mathrm{M}$ & \\
\hline 2 & 34.795 & 39046 & 674 & 0.528 & & $\mathrm{M}$ & \\
\hline Total & & 7393753 & 183885 & & & &
\end{tabular}

Figure S116. HPLC spectra of S1

\section{$<$ Chromatogram $>$}

$\mathrm{mV} \quad$ Chiralpak AD-H, isopropanol/hexane $=10: 90$, flow: $1.0 \mathrm{~mL} / \mathrm{min}, \lambda=254 \mathrm{~nm}$.

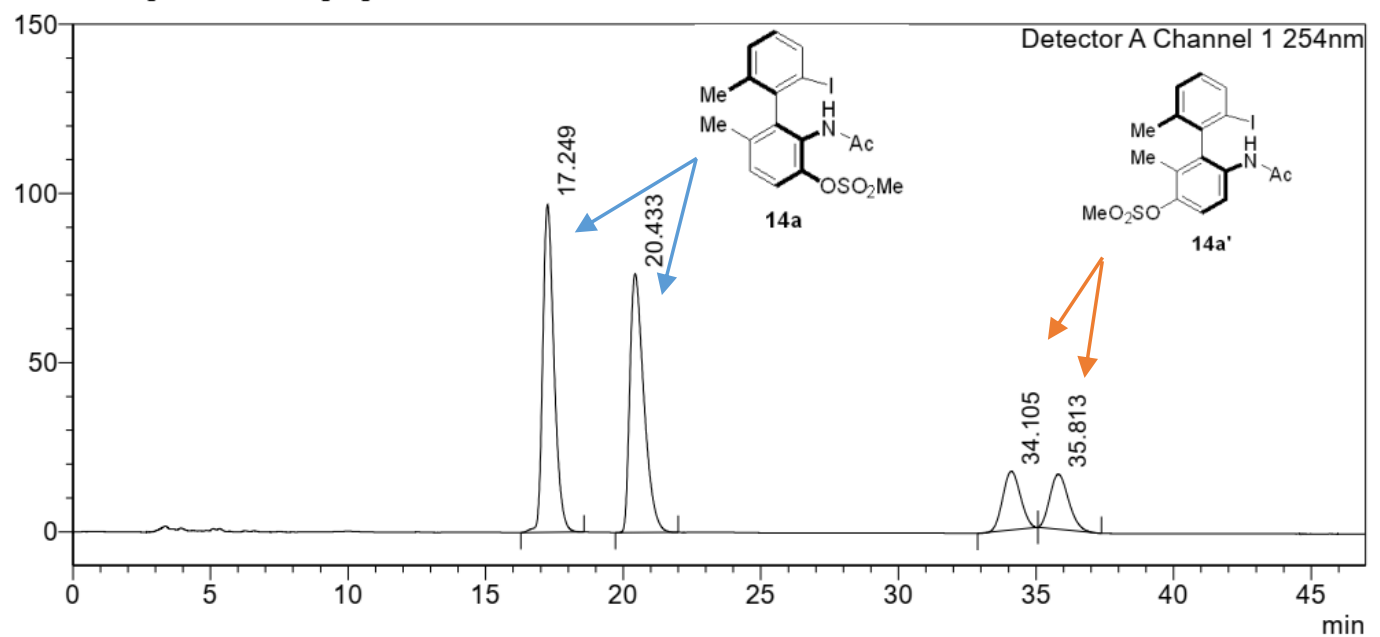

<Peak Table>

Detector A Channel 1254 nm

\begin{tabular}{|r|r|r|r|r|r|r|r|}
\hline Peak\# & Ret. Time & \multicolumn{1}{c|}{ Area } & Height & Conc. & Unit & Mark & Name \\
\hline 1 & 17.249 & 2761731 & 96923 & 39.357 & & $\mathrm{M}$ & \\
\hline 2 & 20.433 & 2713136 & 76473 & 38.664 & & $\mathrm{M}$ & \\
\hline 3 & 34.105 & 771694 & 17347 & 10.997 & & $\mathrm{M}$ & \\
\hline 4 & 35.813 & 770597 & 16295 & 10.982 & & $\mathrm{M}$ & \\
\hline Total & & 7017159 & 207038 & & & & \\
\hline
\end{tabular}

Figure S117. HPLC spectra of 14a\&14a'-rac 


\section{$<$ Chromatogram>}

$\mathrm{mV}$ Chiralpak AD-H, isopropanol/hexane $=10: 90$, flow: $1.0 \mathrm{~mL} / \mathrm{min}, \lambda=254 \mathrm{~nm}$.

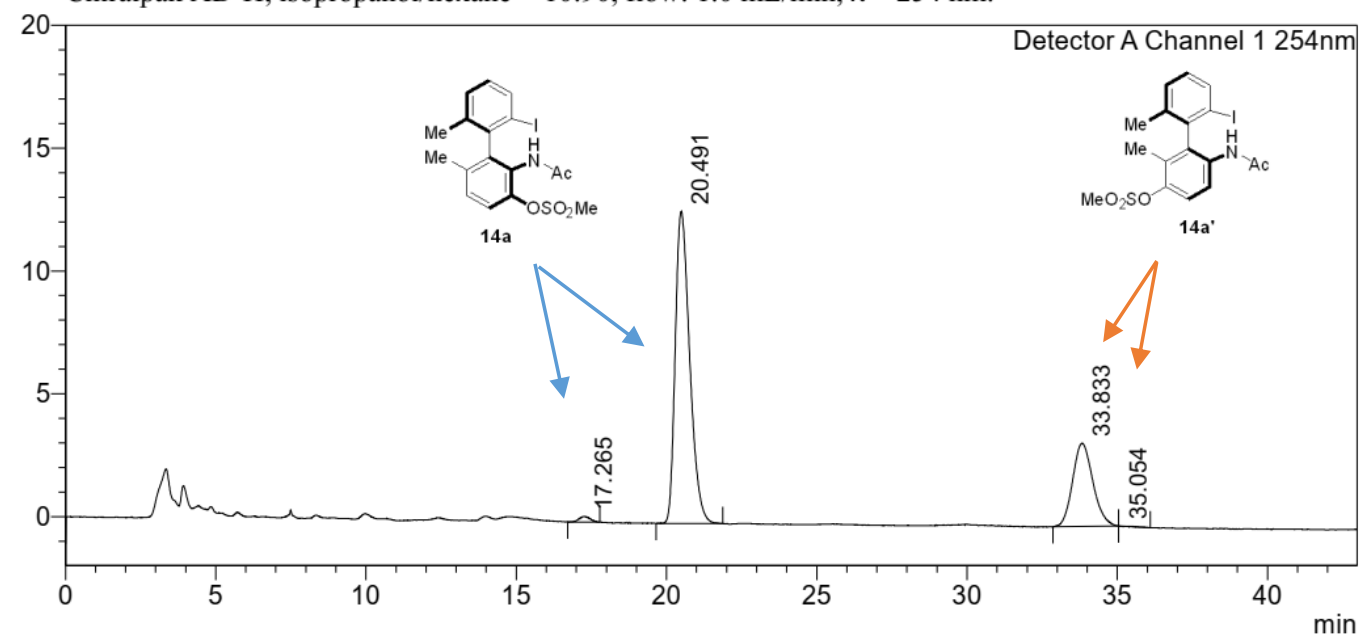

<Peak Table>

Detector A Channel $1254 \mathrm{~nm}$

\begin{tabular}{|r|r|r|r|r|r|r|r|}
\hline Peak\# & Ret. Time & \multicolumn{1}{c|}{ Area } & Height & Conc. & Unit & Mark & Name \\
\hline 1 & 17.265 & 5796 & 226 & 0.982 & & $\mathrm{M}$ & \\
\hline 2 & 20.491 & 424463 & 12709 & 71.955 & & $\mathrm{M}$ & \\
\hline 3 & 33.833 & 159320 & 3383 & 27.008 & & $\mathrm{M}$ & \\
\hline 4 & 35.054 & 325 & -2 & 0.055 & & $\mathrm{M}$ & \\
\hline Total & & 589903 & 16317 & & & & \\
\hline
\end{tabular}

Normalization

\section{$14 a$}

\begin{tabular}{|r|r|r|r|r|r|r|r|}
\hline Peak\# & Ret. Time & \multicolumn{1}{|c|}{ Area } & Height & Conc. & Unit & Mark & Name \\
\hline 1 & 17.265 & 5796 & 226 & 1.347 & & $\mathrm{M}$ & \\
\hline 2 & 20.491 & 424463 & 12709 & 98.653 & & $\mathrm{M}$ & \\
\hline Total & & 430259 & 12935 & & & & \\
\hline
\end{tabular}

14a'

\begin{tabular}{|r|r|r|r|r|r|r|r|}
\hline Peak\# & Ret. Time & \multicolumn{1}{c|}{ Area } & Height & Conc. & Unit & Mark & Name \\
\hline 3 & 33.833 & 159320 & 3383 & 99.796 & & $\mathrm{M}$ & \\
\hline 4 & 35.054 & 325 & -2 & 0.204 & & $\mathrm{M}$ & \\
\hline Total & & 159645 & 3382 & & & & \\
\hline
\end{tabular}

Figure S118. HPLC spectra of $14 a \& 14 a$ ' 
<Chromatogram>

$\mathrm{mV}$

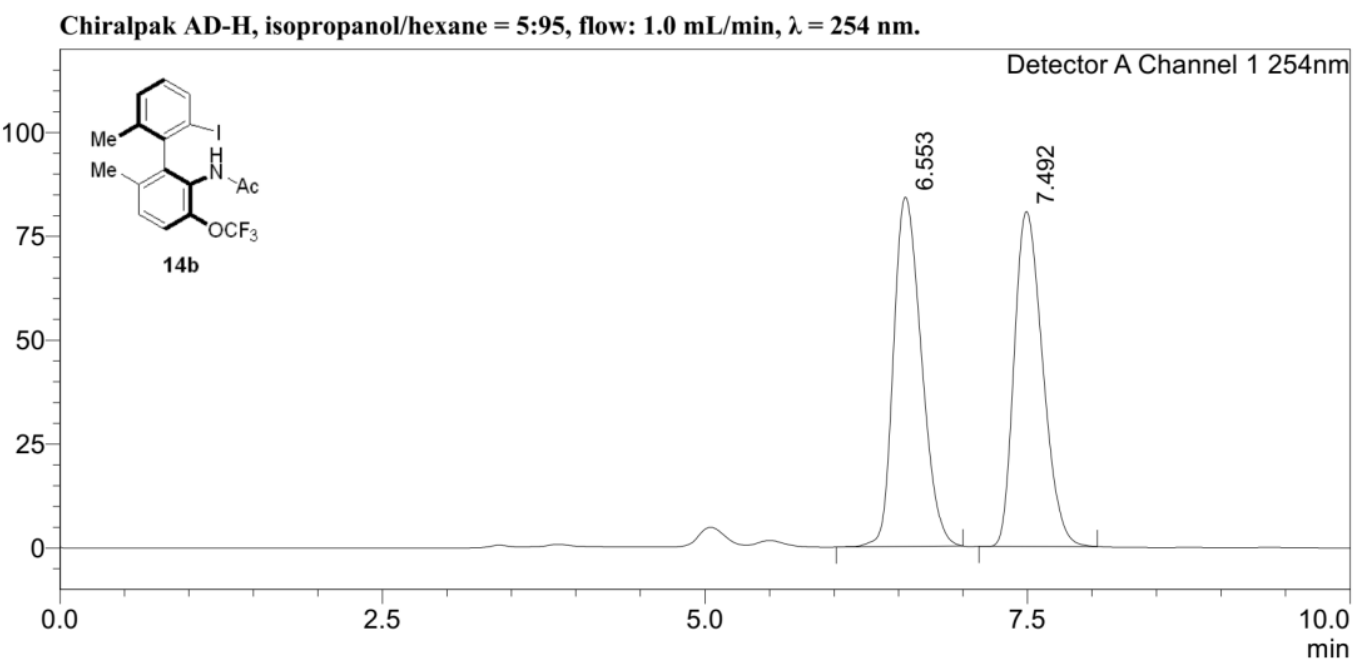

<Peak Table>

Detector A Channel $1254 \mathrm{~nm}$

\begin{tabular}{|r|r|r|r|r|r|r|r|}
\hline Peak\# Ret. Time & Area & \multicolumn{1}{|c|}{ Height } & \multicolumn{1}{|c|}{ Conc. } & Unit & Mark & Name \\
\hline 1 & 6.553 & 1268245 & 84090 & 50.511 & & $\mathrm{M}$ & \\
\hline 2 & 7.492 & 1242580 & 80671 & 49.489 & & $\mathrm{M}$ & \\
\hline Total & & 2510825 & 164760 & & & & \\
\hline
\end{tabular}

Figure S119. HPLC spectra of 14b-rac

\section{$<$ Chromatogram $>$}

$\mathrm{mV}$

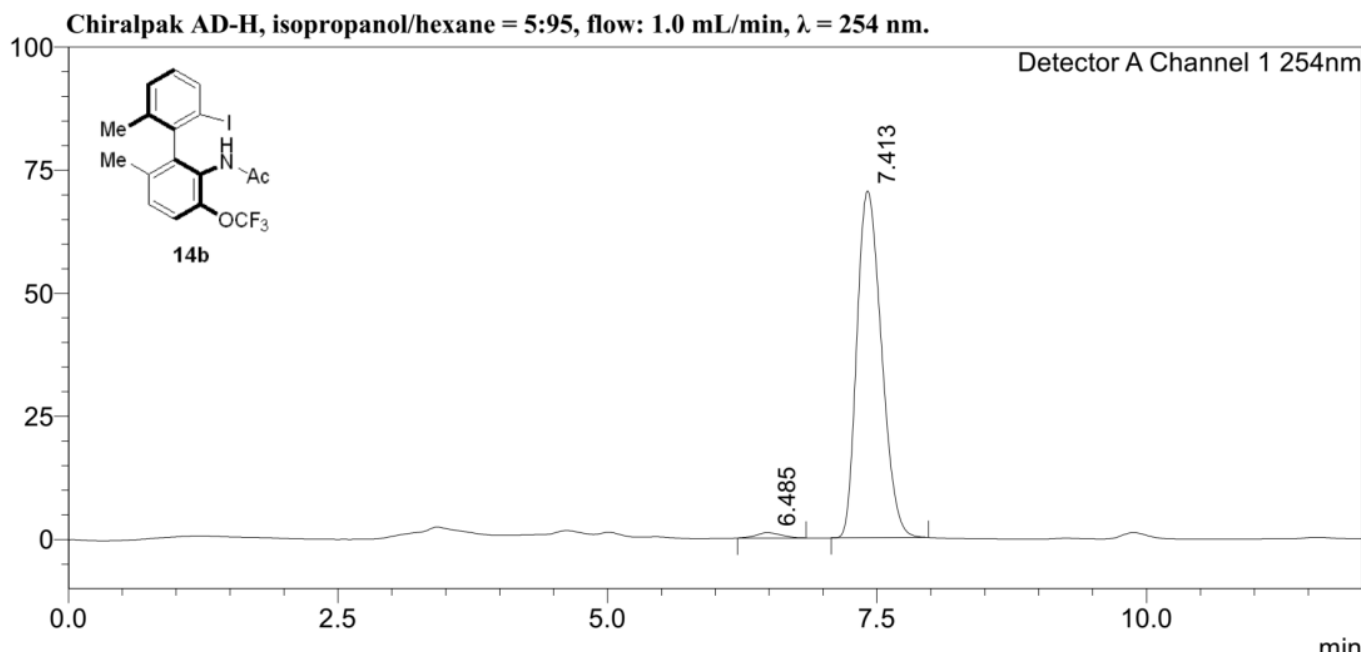

<Peak Table>

Detector A Channel $1254 \mathrm{~nm}$

\begin{tabular}{|r|r|r|r|r|r|r|r|}
\hline Peak\# Ret. Time & \multicolumn{1}{|c|}{ Area } & \multicolumn{1}{|c|}{ Height } & \multicolumn{1}{|c|}{ Conc. } & Unit & Mark & Name \\
\hline 1 & 6.485 & 17332 & 1071 & 1.528 & & $\mathrm{M}$ & \\
\hline 2 & 7.413 & 1117029 & 70439 & 98.472 & & $\mathrm{M}$ & \\
\hline Total & & 1134361 & 71510 & & & & \\
\hline
\end{tabular}

Figure S120. HPLC spectra of 14b 